\title{
Structural elucidation of glycerophospholipids using metastable atom-activated dissociation mass spectrometry (MAD-MS)
}

Robert E. Deimler

West Virginia University

Follow this and additional works at: https://researchrepository.wvu.edu/etd

\section{Recommended Citation}

Deimler, Robert E., "Structural elucidation of glycerophospholipids using metastable atom-activated dissociation mass spectrometry (MAD-MS)" (2014). Graduate Theses, Dissertations, and Problem Reports. 561.

https://researchrepository.wvu.edu/etd/561

This Dissertation is protected by copyright and/or related rights. It has been brought to you by the The Research Repository @ WVU with permission from the rights-holder(s). You are free to use this Dissertation in any way that is permitted by the copyright and related rights legislation that applies to your use. For other uses you must obtain permission from the rights-holder(s) directly, unless additional rights are indicated by a Creative Commons license in the record and/ or on the work itself. This Dissertation has been accepted for inclusion in WVU Graduate Theses, Dissertations, and Problem Reports collection by an authorized administrator of The Research Repository @ WVU.

For more information, please contact researchrepository@mail.wvu.edu. 
Structural elucidation of glycerophospholipids using metastable atom-activated dissociation mass spectrometry (MAD-MS)

\title{
Robert E. Deimler
}

\author{
Dissertation submitted \\ to the Eberly College of Arts and Sciences \\ at West Virginia University
}

in partial fulfillment of the requirements for the degree of

Doctor of Philosophy in Chemistry

Glen P. Jackson, Ph. D., Chair

Stephen J. Valentine, Ph. D.

Harry O. Finklea, Ph. D.

Suzanne C. Bell, Ph.D.

Patrick S. Callery, Ph.D.

Department of Chemistry

\section{Morgantown, West Virginia \\ 2014}

Keywords: Metastable Atom-Activated Dissociation, MAD, LAESI, Lipids

Copyright 2014 Robert E. Deimler 


\title{
ABSTRACT \\ Structural elucidation of glycerophospholipids using metastable atom-activated dissociation mass spectrometry (MAD-MS)
}

\author{
Robert E. Deimler
}

Recent developments in analytical instrumentation have allowed scientists to investigate systems that were unattainable in the past. In the biomedical community this increase in investigative power has allowed the exploration of biological components like proteins, lipids, and DNA and their role in the many different cycles necessary for human life. By understanding the nuances of these processes, medical professionals can more easily recognize the products of mutations or malfunctions that can lead to more accurate prognosis and diagnosis of disease. Typically, lipids are analyzed through hyphenated chromatography-mass spectrometry instruments and especially using collision-induced dissociation (CID). Low-energy CID typically produces both headgroup and complete fatty acid chain loss, whereas high energy CID causes extensive fatty acid chain fragmentation. High energy CID is less widespread, so researchers continually investigate alternate techniques like alkali metallation and multi-stage mass spectrometry to probe the structure of diverse classes of lipids.

Through this work we seek to contribute to this advancement of the biomedical field through the development of a new mass spectrometric fragmentation technique known as metastable atom activated dissociation (MAD). The application of this technique to biological molecules has been demonstrated in the past through peptides, but this is the first application to lipid ions. MAD functions by exposing isolated and stored lipid ions with a beam of high-energy helium metastable atoms. Penning ionization of the lipid ions causes fragmentation throughout the lipid molecules and provides many radical-induced and high-energy products that are not typically observed through collisional activation. MAD is therefore useful in that it can generate high energy fragmentation products in a low-energy environment, like an ion trap, causing unique and extensive fragmentation of lipid molecules without the need for alternate sample preparation steps or multiple stages of mass spectrometry. These abilities allow MAD to contribute unique information about lipid species using a technique that is easy to perform and built using mass spectrometry hardware common to many laboratories. The combination of MAD with other traditional or experimental fragmentation techniques may allow for more informative analysis and a more complete understanding of lipid functionality.

This dissertation also describes the application of a new ambient ionization technique known as laser ablation electrospray ionization (LAESI) to drug identification in a variety of forensically relevant matrices. Currently, the most popular technique for confirmatory analysis in crime laboratories is gas chromatography mass spectrometry (GC/MS). However, the slow analysis times and extensive sample preparation steps have caused significant backlogs in forensic labs. Through this work it was determined that LAESI-MS/MS could successfully detect a wide range of drugs of abuse in various 
media including solutions, hair, and plant matter. LAESI-MS/MS was able to identify the presence of each drug in the test set at both $1 \mathrm{mg} / \mathrm{mL}$ and $5 \mathrm{mg} / \mathrm{mL}$ except phenobarbital, which was only identified at $1 \mathrm{mg} / \mathrm{mL}$. LAESI-MS/MS was also able to identify these drugs in solutions in which a presumptive color test had been performed. In hair and plant samples LAESI-MS/MS was able to identify drugs at biologically relevant levels. Data from these analysis could also be used to generate 2-D ion maps showing the distribution of the drug across the samples. The rapid analysis time of $\sim 5 \mathrm{~s}$ per sample and minimal sample preparation make LAESI-MS/MS a potential candidate for reducing backlogs in crime labs. 
Dedicated to my parents, whose constant love and support made this work possible. 


\section{ACKNOWLEDGEMENTS}

I would like to start off by thanking my advisor, Dr. Glen Jackson, for investing his time and knowledge in my development so I could become an effective chemist. His practical instruction and patient explanation have helped me to not only comprehend difficult theoretical topics but has also allowed me to develop a pool of knowledge and skills that I will continue to draw from for the rest of my life.

At Ohio University there were several individuals who contributed to my success. The first person I would like to thank is Dr. Shannon Cook who spent a great deal time teaching me many of the important concepts that would be prevalent not only in my research but also in my teaching as well. Her patience and willingness to answer my questions no matter how insignificant will always be remembered. The second is Bascom French, whose mechanical knowledge helped to find solutions for repairing temperamental equipment as well as being a source of general advice for any of the many unique problems that can occur during a research experiment. The third is Paul Schmittauer whose electrical expertise helped to troubleshoot many of the problems that occurred within the software of both the mass spectrometer and the computer itself. His lessons on computer repair and network management were invaluable.

I would like to thank my groupmates Yan An, Feng Jin, Mengliang Zhang, Ayat Bani-Rashaid, PengFei Li, and Kateryna Konstantynova for their scientific expertise, advice and fond memories during my graduate experience. I would also like to thank Dr. William Hoffmann for his willingness to answer questions and assist in solving vexing research related problems both practical and theoretical. 
I would also like to thank the members of my dissertation committee both at Ohio University and West Virginia University for taking time to read through my proposal and dissertation and providing valuable feedback both on the written work as well as on its presentation. These suggestions have helped to strengthen the final product. My committee during my time at Ohio University included Dr. Tadeusz Malinski, Dr. Shiyong Wu, and Dr. Gayle Mitchell while at West Virginia University my committee consisted of Dr. Stephen Valentine, Dr. Harry Finklea, Dr. Suzanne Bell and Dr. Patrick Callery. I would also like to thank the National Science Foundation (NSF) and the Ohio Department of Transportation (ODOT) for their financial support over the course of this work through funding provided by NSF CHE-0745590, NSF CHE-1339153, and grants provided by ODOT for the vegetated biofilter and exfiltration trench projects during our collaboration with the engineering department at Ohio University.

I would also like to acknowledge several collaborations, which were important to the fruition of this work. The first is to thank Dr. Kari Green-Church who lent the Jackson lab a quadrupole ion trap mass spectrometer in a time when it was needed so that our research project could continue. I would also like to thank Protea Biosciences for allowing me to visit on several occasions and use their LAESI instrument. I am especially grateful to Trust Razunguzwa for sharing his time and scientific expertise not only showing me the principles and operation of the instrument but also in solving the different issues that occurred during our experiments. I would also like to thank Callee Walsh for the time she devoted to analyzing the hair samples that I was unable to complete within the limited time available during a single Protea visit. I would also like to thank Madlen Sander for her contribution to the MAD project during her time with our 
group. Her scientific insight and enthusiasm for the material allowed us to move forward in our study of radical lipid fragmentation after a stagnant period. 


\section{TABLE OF CONTENTS}

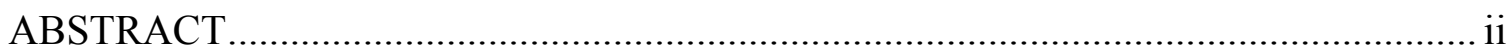

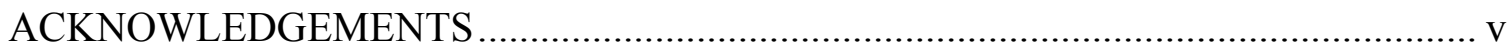

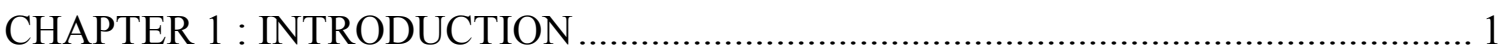

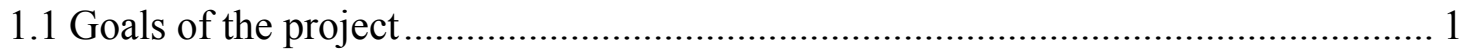

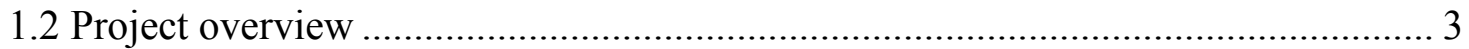

1.3 Ionization techniques for mass spectrometry...................................................... 4

1.3.1 Ionization techniques for the study of lipids.................................................. 5

1.3.2 Ambient ionization of the study of drugs of abuse ......................................... 13

1.4 Quadrupole ion trap mass spectrometry ............................................................ 19

1.4.1 Instrument design .................................................................................... 19

1.4.2 Trapping the ions ………………........................................................... 20

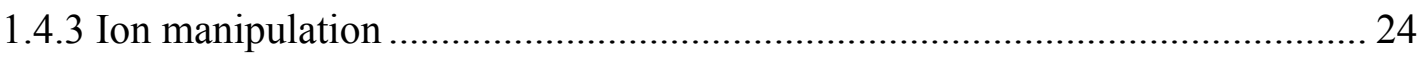

1.5 Mass spectrometry techniques used to study lipids .............................................. 26

1.5.1 Collision induced dissociation (CID) ............................................................. 27

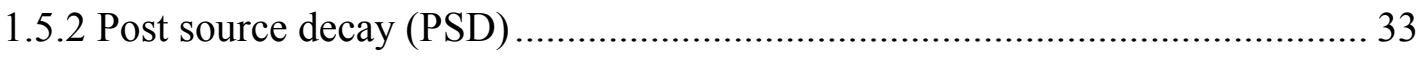

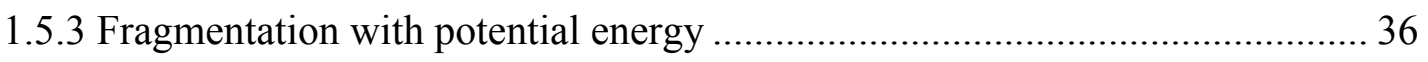

1.6 Metastable atom-activated dissociation (MAD) …………………......................... 37

CHAPTER 2 : INSTRUMENT OPTIMIZATION FOR PRODUCTION OF OPTIMUM

RESPONSE DURING MAD ANALYSIS ............................................................... 45

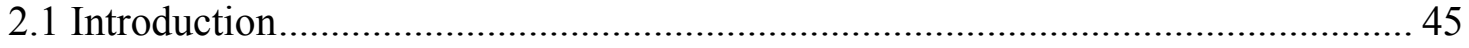

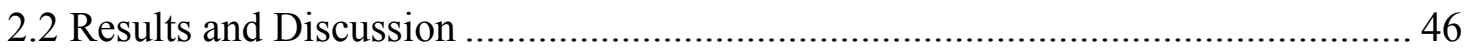

2.2.1 Instrument assessment and course of action ................................................. 46

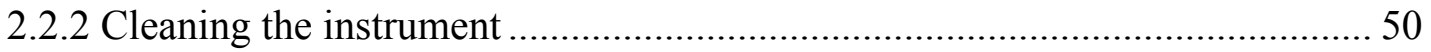

2.2.3 Adjustment of instrumental and MAD variables ............................................ 53

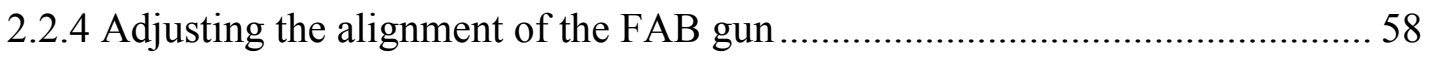

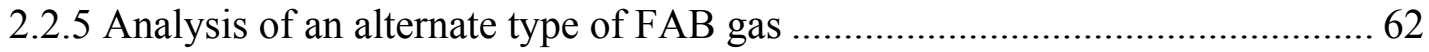

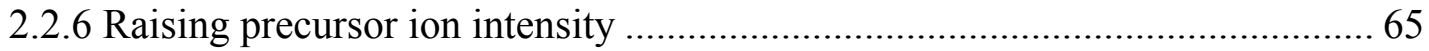

2.2.8 Exploration of an overheating FAB gun ................................................... 70

2.2.9 Successful fragmentation of target lipids....................................................... 73

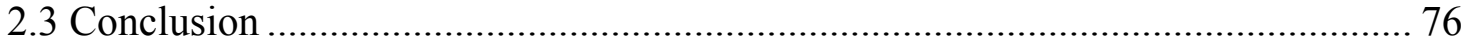


CHAPTER 3 : ANALYSIS OF PHOSPHOCHOLINES USING METASTABLE ATOM ACTIVATED DISSOCIATION MASS SPECTROMETRY (MAD-MS) AND

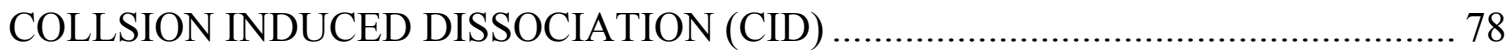

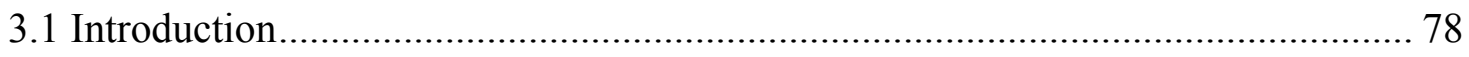

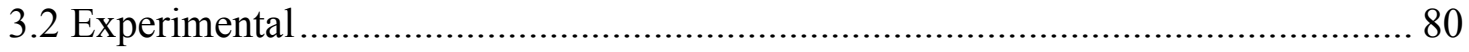

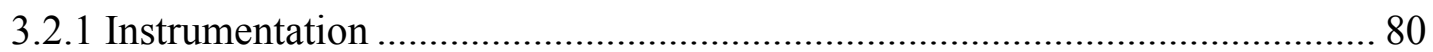

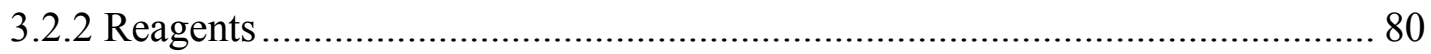

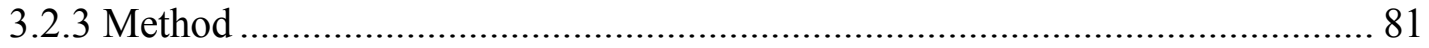

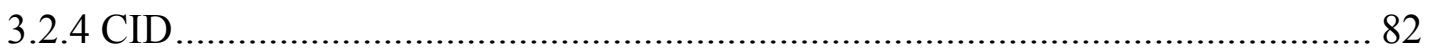

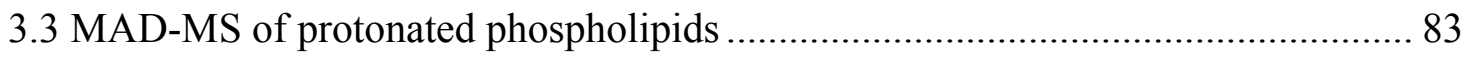

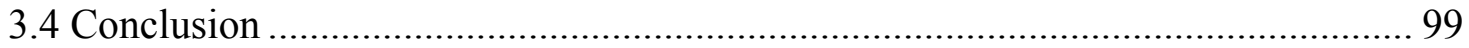

CHAPTER 4 : DIRECT ANALYSIS OF DRUGS IN FORENSIC MEDIA USING

LASER ABLATION ELECTROSPRAY IONIZATION MASS SPECTROMETRY

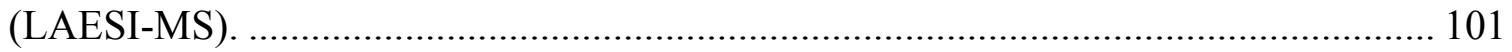

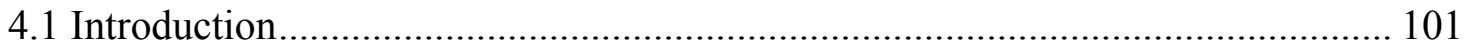

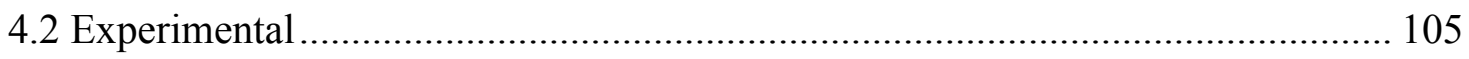

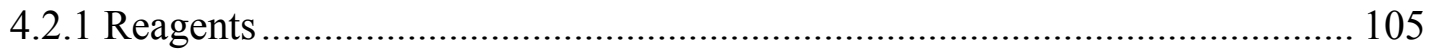

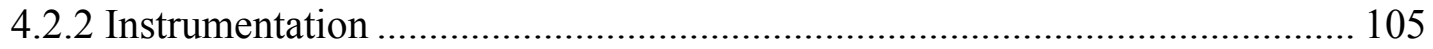

4.3.1 Analysis of drugs in solution after a presumptive color test....................... 108

4.3.2 Analysis of drugs in human hair sample .................................................. 119

4.3.3 Direct analysis of drugs in plant matter ...................................................... 124

CHAPTER 5 : FUTURE WORK AND CONCLUSIONS ........................................ 130

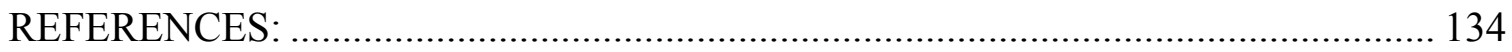


LIST OF TABLES

Table 1.1. Characteristics of Metastable Atoms generated from Noble Gases ${ }^{a}$..... 40

\section{LIST OF FIGURES}

Figure 1.1. Schematic illustrating the ionization of a sample analyte using FAB. (Reprinted with permission from Gross, J. H., Mass Spectrometry: A Textbook. $1^{\text {st }}$ ed.; Springer: Heidelberg, Germany, 2004.).

Figure 1.2. Schematic of an ionization event occurring with MALDI. (Reprinted with permission from Kafka, Alexandra P.; Kleffmann, Torsten; Radesm, Thomas, The application of MALDI TOF MS in biopharmaceutical research. Int. J. Pharm. 2011, 417, 70-82.)

Figure 1.3. Illustration of ESI from the formation of the initial charged droplets to the desolvation and columbic fission steps necessary to get the analyte into the gas phase. (Reprinted with permission from Gaskell, Simon J. Electrospray: Principles and Practices. J. Mass Spectrom. 1997, 32, 677-688.) ...................................... 11

Figure 1.4. Schematic of a DART source where a) is a deflection electrode, b) is the grid electrode and c) is an insulation cap that prevents the operator from being shocked by the electric components. (Reprinted with permission from Alberici, Rosana M.; Simas, Rosineide C.; Sanvido, Gustavo B.; Romao, Wanderson; Lalli, Priscilla M.; Benassi, Mario; Cunha, Ildenize B.S.; Eberlin, Marcos N. Ambient mass spectrometry: bringing MS into the "real world". Anal. Bioanal. Chem. 2010, 398, 265-294.)

Figure 1.5. Schematic showing ionization of sample using DESI. (Reprinted with permission from Alberici, Rosana M.; Simas, Rosineide C.; Sanvido, Gustavo B.; Romao, Wanderson; Lalli, Priscilla M.; Benassi, Mario; Cunha, Ildenize B.S.; Eberlin, Marcos N. Ambient mass spectrometry: bringing MS into the "real world". Anal. Bioanal. Chem. 2010, 398, 265-294.)

Figure 1.6. Illustration of a) how the ion trap functions during operation and b) a cross section of the ion trap. (Reprinted with permission from Gross, J. H., Mass Spectrometry: A Textbook. $1^{\text {st }}$ ed.; Springer: Heidelberg, Germany, 2004.) ....

Figure 1.7. Matheiu stability diagram showing the $\mathrm{q}_{z}$ values in which the ions are stable within the ion trap. In this case any ion with a $\mathrm{q}_{\mathrm{z}}$ value between 0 and 0.908 and a $\beta_{z}$ value between 0 and 1 will be stable. (Reprinted with permission from March, R. E., Quadrupole ion traps. Mass Spectrom. Rev. 2009, 28 (6), 961-989.). 24

Figure 1.8. One example of a system of nomenclature for a specific subclass of lipids. This system was proposed by Adams et. al. for the classification of sphingolipids. (Reprinted with permission from Adams, J.; Ann, Q., Structure determination of sphingolipids by mass spectrometry. Mass Spectrom. Rev. 1993, 12 (1), 51-85.) ... 28 
Figure 1.9. An example of a glycerophospholipid labeled with the common fragments that occur during CID. a)=fatty acid fragments, $b$ ) $=$ the loss of an entire fatty acid chain, and $\mathrm{c})=$ the loss of the phosphocholine headgroup.

Figure 1.10. Schematic of a typical reflectron unit in a time-of-flight mass spectrometer where $L_{1}$ and $L_{2}$ are length of the drift regions, eV is the kinetic energy of the ions, $\mathrm{U}_{0}$ is the kinetic energy distribution of ions of the same type, $\mathrm{s}$ is the distance traveled within the source, $\mathrm{d}$ is the distance travelled into the reflectron, $\mathrm{V}$ is the voltage used to accelerate the ions from the source and $\mathrm{V}_{\mathrm{r}}$ is the voltage applied to the reflectron. (Reprinted with permission from Cotter, Robert J., The new time-offlight mass spectrometry. Anal. Chem. 1999, 71 (13), 445A-451A. Copyright 2014 American Chemical Society.)

Figure 1.11. The molecular energy level diagram of a helium atom showing emission lines that populate the metastable atom levels. (Reprinted with permission from Noto, J.; Kerr, R.; Shea, E.; Waldrop, L.; Fisher, G.; Rudy, R.; Hecht, J.; Gonzalez, S.; Sulzer, M.; Garcia, R., Evidence for recombination as a significant source of metastable helium. J. Geophys. Res.-Space 1998, 103 (A6), 11595-11603.) The ${ }^{1} \mathrm{~S}$ to $2^{1} \mathrm{~S}$ transition of para-helium and the $1^{1} \mathrm{~S}$ to $2^{3} \mathrm{~S}$ transition of ortho-helium are quantum forbidden and result in elevated lifetimes (metastability).

Figure 1.12. Schematic of the position of the cathodes and anode within the end if the FAB gun.

Figure 2.1. Comparison of the isolation and He MAD of $[\mathrm{M}+\mathrm{H}]^{+}$sphingomyelin from a,b) an analysis in September 2011 and c,d) from an analysis in April 2010.......... 48

Figure 2.2. Head-to-tail comparison of the He MAD analysis of $[\mathrm{M}+2 \mathrm{H}]^{2+}$ bradykinin taken in June 2011 (in red) compared with the same analysis performed in September 2011 (in blue).

Figure 2.3. Head-to-tail comparison of the He MAD analysis of $[\mathrm{M}+2 \mathrm{H}]^{2+}$ bradykinin taken in June 2011 (in red) compared with the same analysis performed 1 day after cleaning the instrument (in blue).

Figure 2.4. Comparison of the He MAD analysis of $[\mathrm{M}+2 \mathrm{H}]^{2+}$ bradykinin one day (in blue) 3 days after cleaning the instrument (in red).

Figure 2.5. Comparison of instrumental parameters before and after optimization (top) as well as the He MAD spectrum of $[\mathrm{M}+2 \mathrm{H}]^{2+}$ bradykinin produced by these new parameters (bottom).

Figure 2.6. Comparison of the He MAD spectrum of $[\mathrm{M}+2 \mathrm{H}]^{2+}$ Bradykinin one day (in blue) and three days (in red) after the optimization experiment. 58

Figure 2.7. Comparison of the isolation and He MAD of $[\mathrm{M}+\mathrm{H}]^{+}$of $\mathrm{a}, \mathrm{b}$ ) sphingomyelin and c,d) N-heptadecanoyl-D-erythro-sphingsine. All data taken three days after cleaning the instrument. 
Figure 2.8.Visualization of a) the alignment rod used to center the FAB gun during installation after removing the mass spectrometer's lid and b) the rod in use during an alignment procedure.

Figure 2.9. Photographs and schematics to show a) the way that the alignment tool can shift in the ring electrode, b) the alignment tool once placed in the ring electrode, and c) the ion trap showing the burn mark on the ring electrode.

Figure 2.10. Top-down view of the mass spectrometer a) without the mass spectrometer lid and with colored lines representing each of the lid positions. The red line refers to the left position, the blue line refers to the middle position and the green line refers to the right position as described in the text. Also provided is b) an example of how the system would look with the mass spectrometer's lid on in the left lid position, c) a photograph of the three alignment lines as drawn on the mass spectrometer and d) a photograph of the mass spectrometer lid aligned in the left position.

Figure 2.11. Sample of fragmentation results obtained for MAD to optimize the lid position and MAD conditions: a) the change in $\mathrm{y}_{4}$ with changing FAB voltage $b$ ) the change in $\mathrm{x}_{6}$ with changing pressure $\mathrm{c}$ ) the change in $\mathrm{a}_{5}$ with respect to pulse width and $\mathrm{d}$ ) the change in $\mathrm{x}_{6}$ with respect to pulse width.

Figure 2.12. The Ar MAD spectrum of a) $[\mathrm{M}+\mathrm{H}]^{+}$bradykinin after optimization and b) $[\mathrm{M}+\mathrm{H}]^{+}$sphingomyelin utilizing the optimized parameters.

Figure 2.13. An example of a set of full scan ESI spectra produced during the solution chemistry experiments. This set of spectra shows a) the comparison of the full scan ESI spectrum of a $60 \mu \mathrm{M}$ sphingomyelin made in a 9:1 MeOH/ $\mathrm{H}_{2} \mathrm{O}$ solution, b) a 60 $\mu \mathrm{M}$ sphingomyelin made in a $1: 1 \mathrm{MeOH} / \mathrm{H}_{2} \mathrm{O}$ solution, c) a $20 \mu \mathrm{M}$ sphingomyelin made in a $9: 1 \mathrm{MeOH} / \mathrm{H}_{2} \mathrm{O}$ solution, and d) a $80 \mu \mathrm{M}$ sphingomyelin made in a 9:1 $\mathrm{MeOH} / \mathrm{H}_{2} \mathrm{O}$ solution.

Figure 2.14. Schematic showing the location of the resistor and protective sleeve within

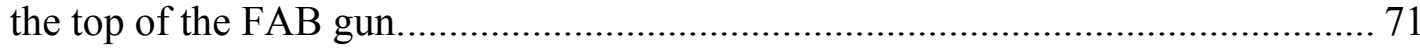

Figure 2.15. Several pictures taken while cleaning the anode of the FAB gun. a) The dirty anode in its place in the nose of the FAB gun before being removed. b) A microscopic image of the contamination coating the top of the anode. c) The anode after the left half has been cleaned. d) The final state of the anode after it has been completely cleaned.

Figure 2.16. Schematic of the duty cycle of the mass spectrometer (in purple), and the experimental pulse times (in red) and widths (in green). The percentage of the duty cycle that the FAB gun is on for each set of experimental parameters is noted on the right.

Figure 2.17. Comparison of the He MAD of $[\mathrm{M}+\mathrm{Na}]^{+}$form of sphingomyelin using data taken from June 2011 (in blue) and April 2010 (in red). 
Figure 2.18. The He MAD of $[\mathrm{M}+\mathrm{H}]^{+} \mathrm{POPC}$ taken with the older Bruker Esquire mass spectrometer at the conclusion of all of the maintenance experiments.................. 75

Figure 2.19. The He MAD of $[\mathrm{M}+\mathrm{H}]^{+}$POPC taken with the new mass spectrometer shortly after transferring to the new university.

Figure 3.1. Comparison of a) He MAD spectrum and b) CID spectrum of the $[\mathrm{M}+\mathrm{H}]^{+}$ precursor of 9E DOPC. Spectra c) and d) show magnified versions of the CID and He MAD spectra respectively, in order to highlight areas of interest. Both the MAD and CID spectra include a diagram of the lipid showing possible homolytic fragment masses. The masses in the inserts may not agree with those shown in the spectra due to hydride shifts or other reactions occuring during fragmentation. 83

Figure 3.2. Comparison of the He MAD of lipids with an increasing amount of unsaturation including a) PSPC, c) POPC, and e) DAPC. Areas of interest are also magnified for each of the spectra in b), d) and f). Fragmentation keys in the bottom corner show the theoretical masses of homolytic cleavage without any $\mathrm{H}$-atom transfers. The masses in the inserts may not agree with those shown in the spectra due to hydride shifts or other reactions occuring during fragmentation.

Figure 3.3. The four and five peak fragmentation patterns located within the chargeremote-fragmentation-like fragmentation cascade occurring during He MAD of POPC.

Figure 3.4. He MAD spectra of a) protonated (9Z) DOPC. Plot b is zoomed in on areas of interest in plot a. The inset shows possible cleavages and theoretical masses for homolytic fragmentation with no H-atom transfers. The masses in the insert may not agree with those shown in the spectra due to hydride shifts or other reactions occurring during fragmentation. 95

Figure 3.5. Comparison of the a) CID spectrum and b) He MAD spectrum of the $[\mathrm{M}+\mathrm{H}]^{+}$ form of 18:0 sphingomyelin (SM).

Figure 4.1. Photograph of a 96-well plate taken with Protea's built in camera prior to analysis using LAESI-MS. The colored wells either contain drug and reagent or a blank of a color test reagent. The red square indicates the reaction of $2.5 \mathrm{mg} / \mathrm{mL}$ each of cocaine and aspirin with cobalt thiocyanate and the blue square indicates the reaction of a $5 \mathrm{mg} / \mathrm{mL}$ cocaine with cobalt thiocyanate. Green squares indicate other wells of interest within the well-plate. All the wells contain solution, but not all are expected to be colored.

Figure 4.2. An example of the comparison between the experimental and NIST reference data for a positive identification of amethamphetamine standard. The experimental data (top in red) is shown head-to-tail with the NIST reference spectrum (bottom in blue). 
Figure 4.3. Summary of the positive identifications for each drug displaying the percentage of all the concentrations of a drug identified in each reagent and displaying the percent of all the reagents a drug is identified.

Figure 4.4. Plot of the number of positive drug identifications versus the $\mathrm{pKa}$ of the conjugate acid form of the individual drugs. Each drug and their corresponding $\mathrm{pKa}$ are identified in the inset table.

Figure 4.5. Comparison of a) full-scan MS spectra of 50:50 quinine $(\mathrm{m} / \mathrm{z} 325)$ and cocaine $(\mathrm{m} / \mathrm{z} 304)$ as well as b) the tandem MS of the $\mathrm{m} / z 304$ cocaine precursor ion and c) the tandem MS spectrum of the $m / z 325$ quinine precursor ion.

Figure 4.6. Calibration curves generated from the peak area of the tandem mass spectrum of a) raw methamphetamine signal and b) the ratio of raw methamphetamine peak area to the raw peak area of the deuterated methamphetamine. Each calibration curve is shown with the $95 \%$ confidence interval of the linear regression line...... 118

Figure 4.7. Head-to-tail comparison of the tandem MS spectra obtained directly from human hair a) of codeine at $\sim 7 \mathrm{ng} / \mathrm{mg}$ (precursor $\mathrm{m} / \mathrm{z} 300$ ) and b) morphine at $\sim 12$ $\mathrm{ng} / \mathrm{mg}$ hair (precursor $m / z$ 286). In each spectrum, the experimental data is shown in red while the NIST reference is shown in blue.

Figure 4.8. Overlay of the ion map of morphine onto the photographic image of the associated hair sample. The color scale associated with each of the ion maps indicates the absolute intensity of the product for the transition from $\mathrm{m} / \mathrm{z} 286 \rightarrow \mathrm{m} / \mathrm{z}$ 201

Figure 4.9. MS/MS data for the analysis of THC in a cannabis leaf a) with LAESI and $b$ ) with DAPPI. (Reprinted with permission from Kauppila, Tiina J.; Arvola, Ville; Haapala, Markus; Pol, Jarolslav; Aalberg, Laura; Saarela, Ville; Framssila, Sami; Kotiaho, Tapio; Kostiainen, Risto. Direct analysis of illicit drugs by desorption atmospheric pressure photoionization. Rapid Commun. Mass Spectrom. 2008, 22, 979-985.)

Figure 4.10. The comparison of a) the MS data for paraffin wax and b) the MS data for permount solution with c) the typical background obtained during a LAESI analysis.

Figure 4.11. MS/MS data for the analysis of THC in a cannabis leaf mounted using paraffin wax with LAESI.

Figure 4.12. A cannabis leaf held onto a microscope slide with a) permount solution after LAESI analysis and b) paraffin wax before LAESI analysis, their associated ion maps c) for the permount mounted leaf d) for the paraffin mounted leaf and the overlay of the leaf image and its associated ion map for e) the permount mounted leaf and $\mathrm{f}$ ) the paraffin mounted leaf. The color scale associated with each of the ion maps indicates the absolute intensity of the product for the transition from $m / z 315$ $\rightarrow m / z 259$, which is selective for THC \& CBD. 


\section{LIST OF SCHEMES}

Scheme 3.1. Illustration of how MAD could produce the observed CID-like fragments at $\mathrm{m} / z 506$ and $\mathrm{m} / z 522$ in 9E DOPC through the Penning ionization fragmentation pathway.

Scheme 3.2. An example of the radical fragmentation method that occurs in lipids after Penning ionization where a) $\star$ indicates the most likely points of attack by a metastable atom, and b) illustrates that radical migration is limited to only a few positions before causing fragmentation.

Scheme 3.3. An example of one doubly charged fragment formed from DOPC upon attack of the double bond by a metastable atom during MAD

Scheme 3.4. Illustration of the radical fragmentation path instigated by Penning ionization which could occur in a highly unsaturated lipid like DAPC. 


\section{LIST OF ABBREVIATIONS}

API

CAE Capillary Array Electrophoresis

CID Collision Induced Dissociation

CRF Charge Remote Fragmentation

DAPPI Desorption Atmospheric Pressure Photoionization

DART Direct Analysis in Real Time

DAPC Diarachidoyl Phosphocholine

DESI Desorption Electrospray Ionization

DOPC Dioleoyl Phosphocholine

ECD Electron Capture Dissociation

ESI Electrospray Ionization

ETD Electron Transfer Dissociation

FAB Fast Atom Bombardment

FAME $\quad$ Fatty Acid Methyl Ester

FT-ICR Fourier Transform Ion Cyclotron Resonance

GC-MS Gas Chormatography Mass Spectrometry

HLPC-MS High Performance Liquid Chromatography Mass Spectrometry

IMS Ion Mobility Spectrometry

IR Infrared

LAESI Laser Ablation Electrospray Ionization

LA-ICP-MS Laser Ablation Inductively Coupled Plasma Mass Spectrometry 


\begin{tabular}{|c|c|}
\hline LTQ & Linear Ion Trap \\
\hline MAD & Metastable Atom-Activated Dissociation \\
\hline MALDI & Matrix Assisted Laser Desorption \\
\hline MIES & Metastable Induced Electron Spectroscopy \\
\hline MIDI & Metastable Induced Dissociation of Ions \\
\hline MS/MS & Tandem Mass Spectrometry \\
\hline NIST & National Institute of Standards and Technology \\
\hline PCA & Principal Component Analysis \\
\hline PI & Penning Ionization \\
\hline PIES & Penning Ionized Electron Spectroscopy \\
\hline PLS & Partial Least Squares \\
\hline PMMA & Polymethylmethacrylate \\
\hline POPC & Phosphotidylcholine \\
\hline PSD & Post Source Decay \\
\hline PT & Pulse Time \\
\hline PTFE & Polytetrafluorethylene \\
\hline PW & Pulse Width \\
\hline QIT & Quadrupole Ion Trap \\
\hline SIMS & Secondary Ion Mass Spectrometry \\
\hline THC & Tetrahydrocannibinol \\
\hline TLC & Thin Layer Chromatography \\
\hline TOF & Time of Flight \\
\hline UV & Ultraviolet \\
\hline
\end{tabular}




\section{CHAPTER 1 : INTRODUCTION}

\subsection{Goals of the project}

The major goal of this project is to develop a new radical-based fragmentation method for the dissociation of precursor ions within a quadrupole ion trap (QIT). One area in which new analytical techniques are frequently adopted is in biomedical research. In this area, new instrumentation allows a more complex and comprehensive view into human biological functions.

One new fragmentation technique being currently being developed is metastable atom-activated dissociation mass spectrometry (MAD-MS). MAD utilizes Penning ionization (PI), which causes fragmentation throughout the target ion after the ionization of one of its electron-rich groups. One ramification of PI is that the initiation of fragmentation is not necessarily dependent on the location of the original charge within the precursor ion, nor is it limited by the initial charge state of the precursor. MAD, then, should be applicable to a variety of compounds of varying makeup and charge state.

Traditionally, collision induced dissociation (CID) has been used as the major mode of tandem mass spectrometry with which to investigate biological ions. CID is an ergodic technique meaning that the energy transferred to the molecule through multiple random collisions will randomize over the entire compound before exceeding a threshold dissociation pathway. CID typically results in the fragmentation of the most labile bonds within a compound. In contrast, MAD is a nonergodic technique that causes radical fragmentation in a similar fashion to electron capture dissociation (ECD) ${ }^{1-4}$ or electron transfer dissociation (ETD). ${ }^{5-8}$ This means that any excess energy leftover from the 
interaction with the metastable atom can cause fragmentation in the vicinity of the group that was ionized. Because metastable atoms are attracted to electronegative groups and high electron density, they are able to activate precursor ions at different sites, independent of the site of protonation. Therefore, the most labile bond around one of these attack points can vary from the most labile bonds for the compound as a whole, leading to different fragmentation patterns than CID. Finally, CID also relies on multiple stages of mass spectrometry to achieve complete fragmentation of target lipids, typically through the use of tandem-in-time or tandem-in-space instruments like a quadrupole ion trap or a triple quadrupole mass spectrometer respectively. In such, experiments $\mathrm{MS}^{3}$ is normally required to observe complete fragmentation of the fatty acid chain.

In past experiments, MAD has been chiefly used to investigate the structure of peptides and proteins. ${ }^{9-11}$ The groups of Zubarev, Berkout and Jackson have found that MAD is able to successfully fragment peptides and proteins producing fragmentation consistent with both radical (e.g c/z) or even electron (e.g. b/y) fragmentation. MAD was also shown to be effective in fragmenting both singly and multiply charged molecules, ${ }^{9,10}$ identifying the difference between isobaric amino acids, ${ }^{9}$ and unambiguously identifying posttranslational modifications. ${ }^{11}$ The successful analysis of these peptides helped to demonstrate the potential of MAD as a tool for the study of biological ions.

After demonstrating that MAD could effectively fragment one family of biomolecules it was decided to expand the scope of MAD and determine if it could effectively fragment other classes of biomolecules as well. In this work we will focus on one prominent and diverse class of biomolecules; lipids. The study of lipids will provide information about the fundamental way that metastable-atom activated dissociation 
(MAD) functions as a fragmentation technique as well as providing an assessment of the practicality and versatility of this technique as it is applied to the study of another group of compounds of interest to the biomedical community. We propose that MAD can provide unique or complementary fragmentation compared to current techniques like collision-induced dissociation (CID) using tandem mass spectrometry rather then the $\mathrm{MS}^{3}$ normally required for complete fragmentation of the lipid.

\subsection{Project overview}

At the beginning of the project, the goal was to work on the MAD system itself to diagnose malfunctions that were preventing the instrument from operating reliably. The MAD system had been continually developed by previous group members who had worked on this project prior to my initial experiments. These problems were characterized by issues ranging from software errors and overcurrents to the inability to collect new data or accurately reproduce prior data. Chapter 2 covers a more in-depth look at the steps taken to identify and correct some of these problems.

The next step after repairing the instrument was to use MAD to study the fragmentation pattern of singly-charged glycerophospholipids. Singly protonated lipids were dissociated successfully using this technique and provided extensive fragmentation. Fragmentation of singly charged precursors is one area in which MAD excels because other radical based techniques do not work with the $1+$ charge state. Initially, the main focus was on the analysis of singly charged lipids both because it is an area where MAD has the potential to contribute but also because many glycerophospholipids do not easily retain multiple charges. These singly charged lipids included those with varying chain lengths (16 to 20 carbon atoms), and degrees of unsaturation ( 0 to 8 double bonds). Each 
lipid was studied to determine both the extent to which MAD could fragment lipids overall and also how the differences in structure changed the fragmentation we observed using MAD. These results were also compared to current techniques like CID and are detailed in Chapter 3.

In addition to investigating MAD of lipids, I was also engaged in an opportunity to work on other projects, such as a collaboration with Protea Biosciences Inc. Through this collaboration I was able to explore the application of a recently developed ambient ionization technique, laser ablation electrospray ionization (LAESI), to the field of forensic science. The rapid sample analysis time and minimal sample preparation made LAESI an appealing candidate for the forensic field, which is now mired in sample backlogs. The ability of LAESI to detect drugs of abuse in several different media including solutions, hair and botanic samples were tested and its capability as an effective forensic technique was assessed. The details of this assessment can be found in Chapter 4.

\subsection{Ionization techniques for mass spectrometry}

The first step in a mass spectrometric analysis is to convert the sample from its original state into gas-phase ions. This conversion is accomplished using an ionization source, of which there are many different varieties. Ionization techniques are normally classified by the degree of fragmentation (hard or soft), by the operating pressure range (ambient or vacuum ionization), or by method of ionization (e.g. photoionization or chemical ionization). This work focuses primarily on the investigation of lipids and of drugs of abuse using mass spectrometry. Lipids are generally ionized using nonatmospheric techniques like matrix assisted laser desorption ionization (MALDI) or fast 
atom bombardment (FAB), but, atmospheric pressure ionization (API) techniques like electrospray ionization (ESI) have also been used. It is also very common to use electron ionization (EI), but such applications are almost exclusively applied to volatile derivatives of lipids such as determining the fatty acid composition of a sample by separating and quantifying the amount of fatty acid methy esters (FAMEs) ${ }^{12,13}$ created after esterification. The study of drugs of abuse has typically been performed using electron ionization or ambient techniques like Direct Analysis in Real Time (DART), Desorption Electrospray Ionization (DESI) or Laser Ablation Electrospray Ionization (LAESI). A more in-depth overview of how these techniques work and their application to either lipids or drug analysis will be covered in the following sections.

\subsubsection{Ionization techniques for the study of lipids}

Fast atom bombardment (FAB) was first developed in the early 1980's by Barber and coworkers. ${ }^{14} \mathrm{FAB}$ was developed to overcome the difficulty of converting thermally labile and nonvolatile species from liquids into the gas phase for mass spectrometric analysis. At the time, gas chromatography mass spectrometry (GC-MS) was a popular technique for analyzing biological species like peptides. ${ }^{15}$ The problem with GC-MS was that it mainly used heat to convert the solid or liquid sample into a gas. If the sample was thermally labile, as with many biological samples exceeding the weight of $500 \mathrm{Da}$, the heat would alter the structure or destroy a sample before any information could be obtained. At the time, one common technique for the analysis of surface and thin film analysis was secondary ion mass spectrometry (SIMS).$^{16}$ Over time SIMS was modified to produce other techniques like ${ }^{252} \mathrm{Cf}$ plasma desorption ${ }^{17}$ which used fission products of californium as its ionizing beam rather then the uniform ion beam typically used in 
SIMS. Eventually, SIMS was applied to organic samples and the results were problematic. SIMS tended to cause electrostatic charging of samples and this charging would disrupt the ion source potentials in the mass spectrometer causing the ion signal to deteriorate. ${ }^{18}$ To get around this problem, Barber and coworkers bombarded samples with neutral atoms instead of the ions normally associated with SIMS and eliminated the charging problem. Later, the introduction of a liquid sample matrix would further improve the experimental results obtained with $\mathrm{FAB}$ and make it a major competitor with other techniques of the time like field desorption. ${ }^{19,}{ }^{20} \mathrm{FAB}$ functions by bombarding a target compound with a beam of neutral high energy atoms. (Figure 1.1) This beam is generated using a FAB gun that is normally mounted at an angle over the sample stage. Inside a typical saddle-field FAB gun, an anode is used to generate electrons that ionize the $\mathrm{FAB}$ gas of choice using a process similar to electron ionization (EI).

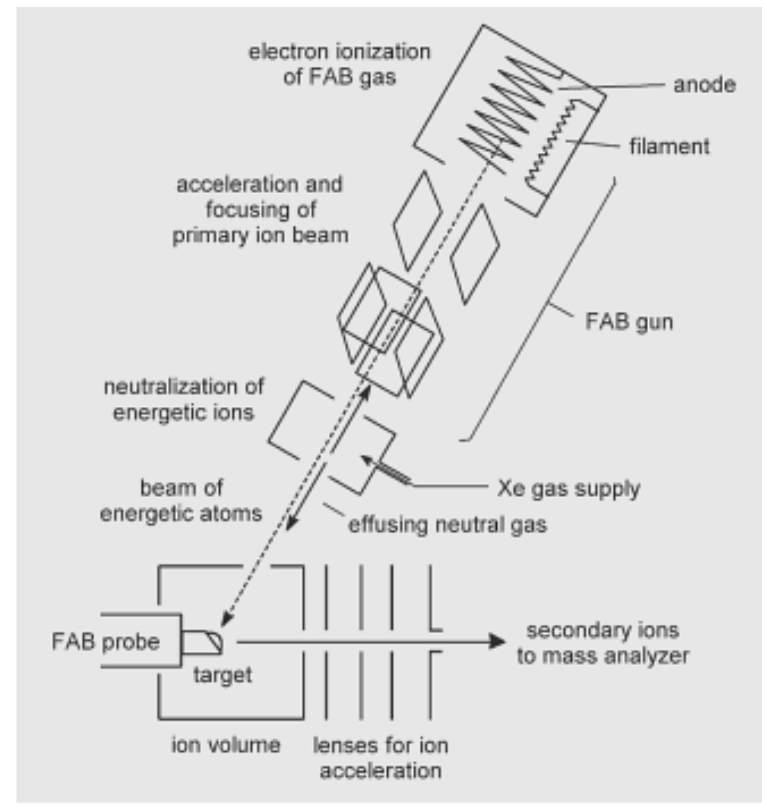

Figure 1.1. Schematic illustrating the ionization of a sample analyte using FAB. (Reprinted with permission from Gross, J. H., Mass Spectrometry: A Textbook. $1^{\text {st }}$ ed.; Springer: Heidelberg, Germany, 2004.) 
A variety of gases have been used for FAB including xenon, ${ }^{21}$ argon, ${ }^{22}$ and neon. ${ }^{23}$ The electrons ionize the FAB gas, which is then accelerated by the same anode potential through the FAB gun using a high voltage in the range of $4-8 \mathrm{kV} .{ }^{18}$ On the way out of the gun, the ions are neutralized through charge exchange with incoming neutral gas atoms which are continually flowing in the bottom of the gun. Charge exchange occurs with little change in the momentum of the atom, thereby allowing the high-kinetic energy atom to continue out of the gun with similar energy to what it had at its initial velocity as an ion. ${ }^{20}$ The high-energy neutral atoms then travel out the bottom of the gun to the sample where they cause desorption and ionization. The mechanism for ionization in FAB is not well understood. Currently, there are two major theories to how ions are formed during FAB analysis, including the chemical ionization model ${ }^{24-26}$ and the precursor ion model. ${ }^{27-29}$ The chemical ionization model is based on the idea that by focusing a high-energy beam of atoms on one spot in the sample, enough thermal energy is created that a plasma is formed. The interaction of the sample and matrix with the plasma then causes the ionization of the sample. The precursor ion model for FAB, on the other hand, is based on the idea that the analytes are already ionized in the sample and that all the FAB beam does is dislodge them from the sample stage and into the gas phase. As mentioned above, FAB is enhanced through the use of a sample matrix. These sample matrices serve many purposes from absorbing and distributing energy — so the sample doesn't experience thermal degradation — to assisting in sample ionization through the formation of electron donating or withdrawing species upon being exposed to FAB ${ }^{18}$ Common matrices used with FAB include glycerol, 3-nitrobenzyl alcohol (NBA) and triethanolamine (TEA). FAB can generate both positive and negative ions, is 
typically associated with species up to 3000 Daltons and is commonly utilized in conjunction with double magnetic sector instruments. ${ }^{18}$ FAB has been used to analyze a variety of lipids including fatty acids, ${ }^{30,31}$ glycerophospholipids, ${ }^{32,33}$ and sphingolipids ${ }^{34}$. Although still in use, FAB is now forced to compete with newer technologies like MALDI or ESI.

The concepts that would eventually become MALDI were first developed in the late 1980's by groups in Japan and Germany. Koichi Tanaka in Japan was able to show that the laser desorption ionization of organic compounds was enhanced through the addition of an "ultra fine metal plus liquid" matrix. ${ }^{35}$ At the same time in Germany, Franz Hillenkamp and Michael Karas observed that the combination of a sample with an organic matrix caused an increase in the intensity of the laser desorption signal. ${ }^{36}$ MALDI was developed in the pursuit of a technique that could ionize molecules with large molecular weights. Popular techniques at the time had reached a limit as to the most massive molecule they could ionize and exploration of larger biomolecules like proteins was difficult. Early experimentation with laser desorption focused on smaller biomolecules like amino acids (molecular weight 57-186 Da) but as MALDI began to develop as its own technique, the applications extended to larger compounds like $\alpha_{1}$-acid glycoprotein (molecular weight $36,800 \mathrm{Da}) .^{35,37,38}$

MALDI causes ionization through the ablation of a matrix-mounted sample using a laser and although there is still debate about the exact mechanism of ionization, there is a consensus that the matrix is essential for photoabsorption and proton transfer to or from analytes. ${ }^{38}$ Figure 1.2 shows a schematic of the MALDI ionization event. ${ }^{39}$ The laser used in a MALDI experiment is usually an ultraviolet (UV) laser although infrared (IR) 
lasers have also been used. ${ }^{18,40}$ The matrix used is dependent on the sample being analyzed. For example, in the analysis of oligonucleotides and DNA, analogues of picolinic acid are commonly used. For peptides, smaller proteins and triacylglycerols $\alpha$ cyano-4-hydroxycinnamic acid is more common. ${ }^{18}$ Acid matrices are normally selected because they help to protonate the analyte before or during the laser ablation process.

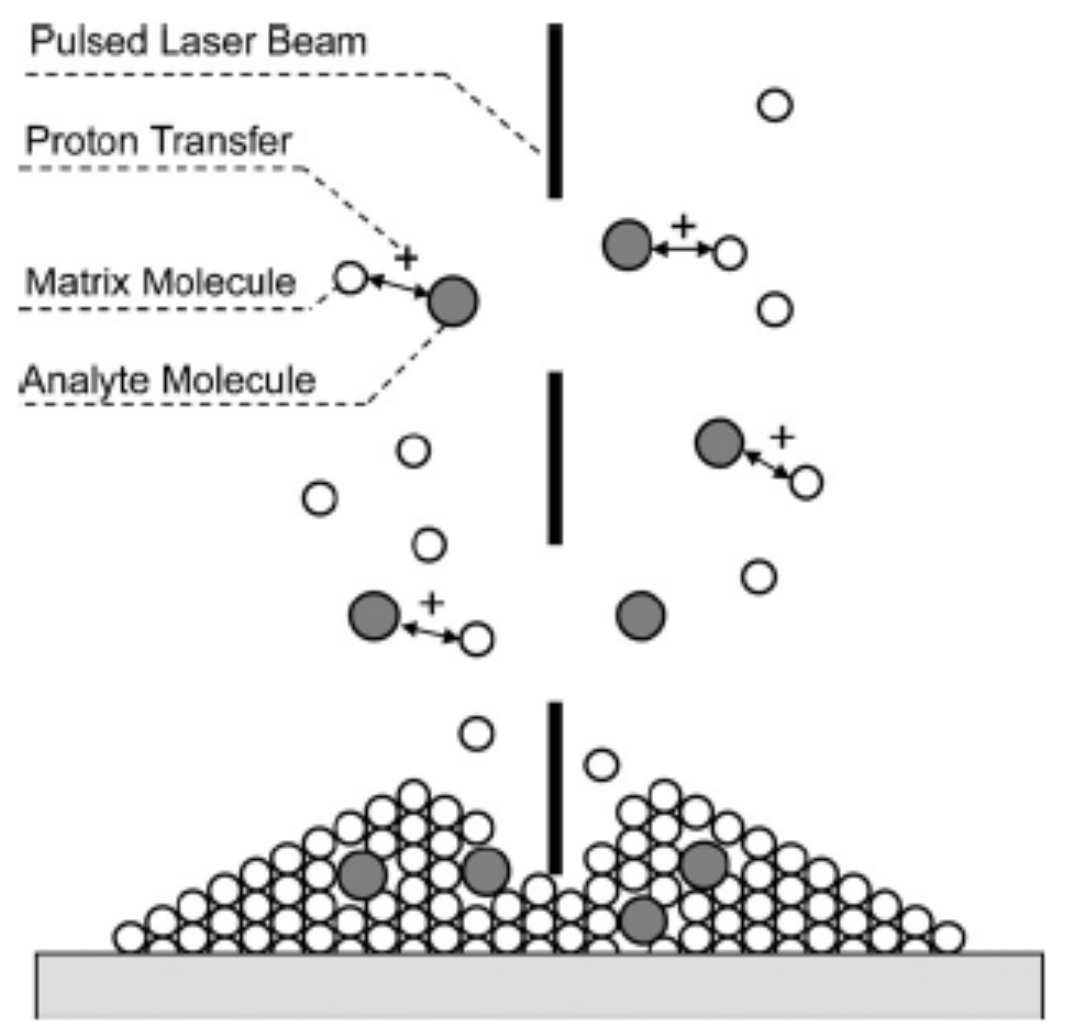

Figure 1.2. Schematic of an ionization event occurring with MALDI. (Reprinted with permission from Kafka, Alexandra P.; Kleffmann, Torsten; Radesm, Thomas, The application of MALDI TOF MS in biopharmaceutical research. Int. J. Pharm. 2011, 417, 70-82.)

Without a matrix, prolonged exposure to the laser beam could cause photodissociation or sample degradation. ${ }^{38}$ The matrix can also help to dilute the sample molecules and prevent them from aggregating together and being too large to ionize. ${ }^{38}$ In 
other words, the matrix can help to prevent clusters of molecules from forming that would be too large to desorb in the absence of the matrix.

Because MALDI focuses on ionizing molecules with molecular masses greater then $1000 \mathrm{Da}$, it is typically coupled with TOF instruments. TOF instruments are selected because they have one of the largest mass ranges of all mass analyzers. One area of research where MALDI has been applied is in the study of lipids, although ESI is more common. However, one reason that MALDI is adopted for lipid analysis is that it is less sensitive to buffer or salt contamination that may plague other techniques. ${ }^{41}$ MALDI has also been used for the study of lipids because of its close association with the TOF mass spectrometer.

There have been several studies focusing on studying the fragmentation pattern of lipids using high energy CID, a fragmentation technique closely associated with TOF. ${ }^{42-44}$ Marshal's group were the first to couple MALDI to Fourier transform ion cyclotron resonance (FT-ICR) mass spectrometry wherein they studied glycophospholipids with different head groups. ${ }^{45}$ Lipid studies have also been performed to try and improve the coupling ${ }^{46}$ between chromatographic techniques like thin layer chromatography (TLC) and MALDI-MS using sphingolipids ${ }^{47}$ and capillary array electrophoresis (CAE) using a peptides as the test compound with MADI-TOF ${ }^{48}$ Several studies with MALDI have focused on the analysis of phospholipids in different media including plants materials and human biological fluids. ${ }^{41,49-51}$

ESI was developed in the late 1980 's by Fenn ${ }^{52-54}$ based on ideas that had been proposed up to seventy years earlier by Dole, ${ }^{55}, 56$ Zeleny, ${ }^{57}$ and Taylor. ${ }^{58}$ ESI was developed in pursuit of the same problem as MALDI. ESI sought to easily ionize large 
non-volatile compounds beyond the scope of other techniques. ESI generates ions by spraying a sample solution through an electrical charged needle (Figure 1.3). ${ }^{59}$ The solution is composed of a mix of volatile solvents like methanol, water or acetonitrile and can have other additives like acetic acid or ammonium hydroxide to aid in protonation or deprotonation of the target analyte. The combination of the electric field and the forward pressure from the pump causes the sample to leave the needle as mist of highly charged droplets, and these droplets are then attracted to the inlet of the mass spectrometer

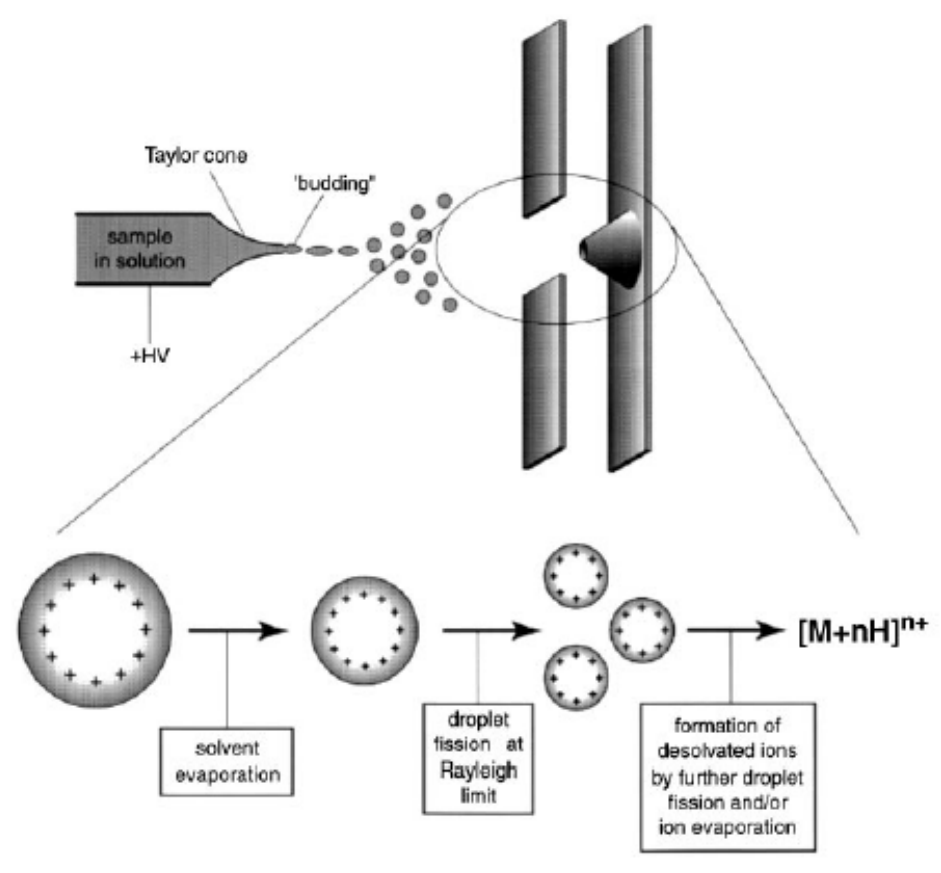

Figure 1.3. Illustration of ESI from the formation of the initial charged droplets to the desolvation and columbic fission steps necessary to get the analyte into the gas phase. (Reprinted with permission from Gaskell, Simon J. Electrospray: Principles and Practices. J. Mass Spectrom. 1997, 32, 677-688.)

through a pressure and electrostatic gradient. As the droplet travels towards the inlet, it starts to undergo desolvation and decreases in size. As the droplet size gets smaller, excess charges in the droplet get closer together until columbic repulsion of the charges is 
greater then the surface tension of the droplet and the droplet explodes into several smaller droplets (Figure 1.3)..$^{59}$

At this point in the ionization process, there is some debate as to how the analyte molecule is finally converted into free ions. This debate mainly centers around two models, the charge-residue model and the ion evaporation model. In the charge residue model the droplet containing the analyte molecule keeps exploding until eventually there is a daughter droplet that contains only a single analyte molecule which is then converted to the gas phase through desolvation of the droplet. ${ }^{18,60,61}$ The ion evaporation model, on the other hand, suggests that when the droplet gets small below $10 \mathrm{~nm}$ that instead of undergoing columbic fission the charges in the droplet will instead repel the like-charged analyte straight out of the droplet and into the gas phase. ${ }^{18,60,61}$ Regardless of which model actually occurs, at the end of this process only the charged gas phase analyte is left to travel into the mass spectrometer for analysis. ESI has an extended mass range compared to other ionization techniques and has been able to successfully analyze large biomolecules ${ }^{62}$ with mass up to $914 \mathrm{kDa} .^{63}$ This ionization technique is also compatible with many different types of mass spectrometers, including magnetic sectors, ${ }^{64}$ FTICRs, ${ }^{65} \mathrm{TOFs},{ }^{66}$ and ion traps. ${ }^{67}$ ESI has been used to study several different types of lipids including sphingolipids, ${ }^{68,69}$ glycerophospholids, ${ }^{68,70-73}$ and triacylglycerols. ${ }^{74}$

In this section I have discussed a number of different ionization sources that have been used to effectively study lipids. This project mainly focuses on utilizing ESI as the ionization method of choice during analysis. However, an in-depth knowledge of how each ion source functions and has been used to study lipids will help in the interpretation and eventual comparison of data generated with our MAD unit to results obtained with 
other combinations of ionization sources and mass spectrometers. Further details about the integration of the ESI source into the overall MAD unit will be discussed in later sections of this work.

\subsubsection{Ambient ionization of the study of drugs of abuse}

Ambient ionization techniques are those that seek to minimize sample preparation through the use of instrumentation that can analyze samples directly in the open atmosphere. The minimal sample preparation, ease of use, and large number of potential applications have led to the development of several techniques within this family including DART (Direct Analysis in Real Time), DESI (Desorption Electrospray Ionization), and LAESI (Laser Ablation Electrospray Ionization).

DART was first described in 2005 by Cody and Laramee after attempts to develop an atmospheric pressure thermal electron source. ${ }^{75}$ They discovered that when they coupled one of their prototype electrical discharge sources into an atmospheric pressure interface of a time of flight mass analyzer (TOF) that they could detect volatile substances from across the laboratory. Further experimentation also showed that they could desorb and ionize compounds directly from a surface too.

DART functions by ionizing the sample analyte directly using a heated stream of metastable atoms. (Figure 1.4) These metastable atoms/molecules can be generated from a variety of gases including nitrogen, neon, and argon, although helium is most commonly used. The gas is pumped into the first stage of the DART source where a plasma is created using a glow discharge. This plasma generates a variety of products including ions, electrons, and neutral metastable species. ${ }^{76}$ These plasma products then travel into the second stage of the DART source where a deflection electrode is set to 
remove the charged products so that only a stream of neutral metastable species are allowed to enter the next stage. In the third stage of the DART source the stream of metastable species is heated to enhance the thermal desorption of the analyte from its matrix. ${ }^{76}$ After being heated, the metastable atoms pass through a grid electrode that removes ions of opposite charge from those blocked by the deflection electrode. ${ }^{77}$ Finally, the metastable atoms exit the DART source and contact with the sample that is normally held between the source and the inlet of the mass spectrometer.

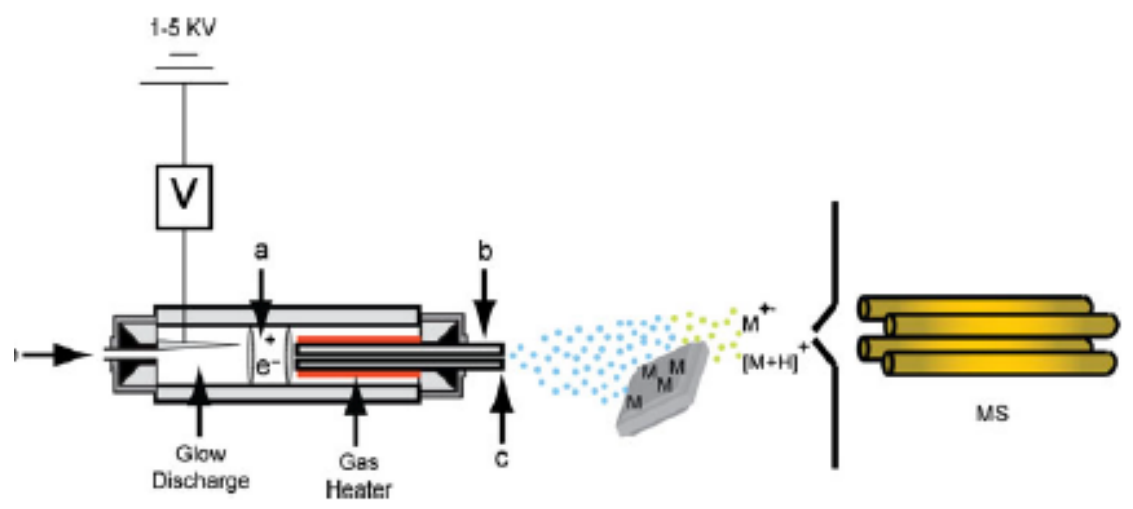

Figure 1.4. Schematic of a DART source where a) is a deflection electrode, b) is the grid electrode and c) is an insulation cap that prevents the operator from being shocked by the electric components. (Reprinted with permission from Alberici, Rosana M.; Simas, Rosineide C.; Sanvido, Gustavo B.; Romao, Wanderson; Lalli, Priscilla M.; Benassi, Mario; Cunha, Ildenize B.S.; Eberlin, Marcos N. Ambient mass spectrometry: bringing MS into the "real world". Anal. Bioanal. Chem. 2010, 398, 265-294.)

Analysis in DART is normally restricted to singly charged species in either the positive or negative mode of analysis. Ionization in DART normally occurs either through Penning ionization, proton transfer (to create positive species) or electron capture (to create negative species). ${ }^{78}$ This method of ionization is effective in ionizing molecules of lower mass present in a sample, typically less than $800 \mathrm{Da}^{76,77,79,80}$ This limited mass range can be good in that it tends to avoid the higher masses normally associated with the 
sample matrix. This range can also be a disadvantage in that it prevents the application of DART to larger analytes.

Typically, DART is coupled to TOF mass spectrometers, but DART can also be effectively coupled to ion mobility spectrometers (IMS) and tandem mass spectrometry (MS/MS) techniques as well. ${ }^{78}$ The power of DART, like other ambient ionization techniques, is in its ability to detect samples that are directly placed into the path of the ionizing beam without extensive sample preparation. For example, Cody and Laramee were able to track the distribution of capsaicin in peppers just by passing parts of the pepper in front of the instrument. ${ }^{75}$ From its inception, this technique was also used for forensic applications as well. One of the first applications of DART was as a way to detect chemical warfare agents for the army. ${ }^{75}$ Other investigative applications soon followed including the using of DART to detect explosives, ${ }^{81}$ drugs, ${ }^{82,83}$ and ignitable liquids. $^{75}$

Around the same time that DART was in development, another technique called DESI was drawing attention in the scientific community. DESI was first discovered in 2004 by a team of researchers led by R. Graham Cooks. ${ }^{84}$ Like DART, DESI ionizes samples under ambient conditions. However, DESI achieves ambient ionization quite differently than DART. As its name would suggest DESI seeks to desorb analytes from a surface using a high-velocity electrospray. (Figure 1.5) DESI functions by applying a voltage to the tip of a syringe that contains the experimental solvent. These solvents are similar to those used in electrospray ionization (ESI) and include solvents like methanol, water or acetonitrile. ${ }^{77}$ The nebulizing system is held at an angle so that the sample pickup droplets produced by the collision of the solvent with the surface will ricochet towards 
the inlet of the mass spectrometer. The nebulizer position and angle are therefore important variables. Another important factor in DESI is the composition of the sample

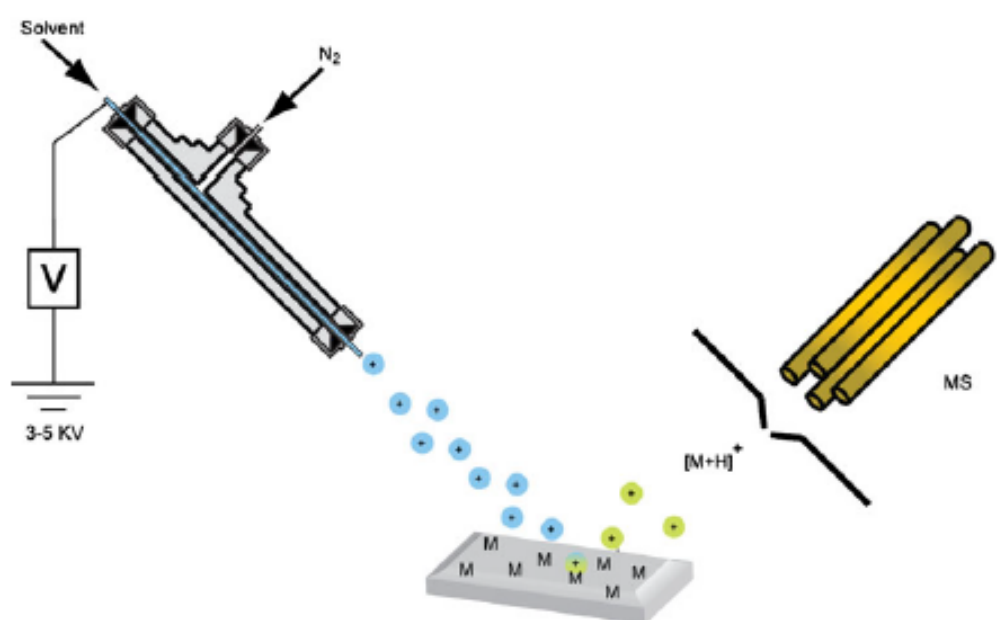

Figure 1.5. Schematic showing ionization of sample using DESI. (Reprinted with permission from Alberici, Rosana M.; Simas, Rosineide C.; Sanvido, Gustavo B.; Romao, Wanderson; Lalli, Priscilla M.; Benassi, Mario; Cunha, Ildenize B.S.; Eberlin, Marcos N. Ambient mass spectrometry: bringing MS into the "real world". Anal. Bioanal. Chem. 2010, 398, 265-294.)

surface. Because DESI works exclusively with charged droplets, it is important that the sample surface is not made of a substance that is electrically conductive that will neutralize them. Common surfaces used for DESI include those made of polytetrafluorethylene (PTFE) for negative mode analysis and polymethylmethacrylate (PMMA) for positive mode analysis. ${ }^{77,85}$ Because the final products of DESI are essentially the same as ESI, any mass spectrometer that can operate with an atmospheric pressure ionization source (API) like ESI, will work with DESI. ${ }^{77}$ DESI has been utilized alongside a variety of mass spectrometers including Orbitraps, Fourier transform ion cyclotron resonance (FT-ICR) and ion mobility spectrometry time of flight (IMS-TOF) 
mass spectrometers. ${ }^{77,86-88}$ Unlike DART, DESI has the ability to analyze larger molecules with some studies successfully ionizing masses up to 66,000 Daltons. ${ }^{77,89}$ This large mass range is an advantage in that it opens DESI up to compounds that may be unattainable with other techniques like DART. This large mass range can also be a disadvantage as well in that it will also be susceptible to matrix interferences that may be avoided with other techniques where lower mass ranges prevent ionization of the matrix. DESI has been used in a variety of forensic applications including detection of illicit drugs, ${ }^{90-92}$ detection of explosives, ${ }^{93-95}$ and imaging and analysis of latent fingerprints. ${ }^{96}$

Shortly after the development of DART and DESI was the development of a plethora of ambient techniques which have been summarized elsewhere. ${ }^{77,97}$ LAESI was first developed by Nemes and Vertes in $2007 .{ }^{98}$ LAESI was developed to address some of the problems associated with other ambient ionization techniques, like the requirement of an external matrix (MALDESI), an ill-defined surface area (DESI) or limited mass range (DART) ${ }^{98}$ LAESI functions by exposing the sample to a $2940 \mathrm{~nm}$ laser. The laser used in the experiment can vary but early LAESI experiments used an Er:Yag laser. ${ }^{98}$ The wavelength of the laser is deliberately tuned to correspond to some of the vibrational states of the $-\mathrm{OH}$ groups in the sample. When the $-\mathrm{OH}$ groups are exposed to the laser they begin to heat and vibrate rapidly until ablation occurs. The ablated sample travels from the sample stage into an ESI spray located above the stage where it is carried into the mass spectrometer for analysis. Because ablation focuses on the excitation of $-\mathrm{OH}$ vibrations the sample must contain at least one component with $-\mathrm{OH}$ bonds. This is normally accomplished through the use of a solvent like water or methanol. 
In the original experiment, LAESI was combined with a TOF mass spectrometer. However, like DESI, any mass spectrometer that has the ability to do API analysis would conceivably be compatible with LAESI. For example, the work in this paper was performed using LAESI and a linear ion trap. LAESI also has the ability to analyze an extended mass range and can analyze samples up to 66,000 Daltons. ${ }^{98}$ The extended mass range opens LAESI to the analysis of a variety of compounds but can also lead to some matrix interference similar to what is seen in DESI. The major advantage of LAESI over other techniques is in its ability to perform high throughput automated analysis. Systems have been designed for LAESI that allow the analysis of entire well plates in minutes through the use of an autosampler. Regarding disadvantages, LAESI can only analyze samples that have $-\mathrm{OH}$ groups present so a sample that lacks these groups or is incompatible with an appropriate solvent cannot be analyzed. LAESI is currently a relatively expensive ion source, mainly because of the limited number of suppliers of pulsed IR lasers. Finally, LAESI is not yet compatible with chromatographic techniques, so is somewhat limited in the dynamic range and classes of analytes that are accessible in complex samples. One major application that LAESI has been applied to so far has been molecular imaging. ${ }^{99}$ LAESI has been used to image metabolites in plants, ${ }^{100}$ as well as in rat brain sections. ${ }^{101}$ Direct studies have also been performed using LAESI on cyanobacteria, ${ }^{102}$ and individual plant cells. ${ }^{103}$

\subsection{Quadrupole ion trap mass spectrometry}

The operating principles of the QIT were first described in 1953 by Paul and

Steinwedel. ${ }^{104-107}$ Further development of these ideas by Paul and his coworkers led to the release of the first commercially available QIT in the 1980's. ${ }^{78,108-110}$ These instruments 
soon became very attractive because they were easy to use, inexpensive, and could easily perform MS/MS experiments. ${ }^{18,67}$ The importance of this technology was further confirmed when Paul was awarded a Nobel prize in $1989 .{ }^{104}$ Today, QITs are used for a variety of applications ranging from environmental to pharmaceutical applications. ${ }^{111}$

\subsubsection{Instrument design}

A QIT is a small cylinder about the size of a baseball that sits inside the vacuum chamber of the mass spectrometer. (Figure 1.6) The QIT consists of two end caps which sandwich a donut shaped ring electrode. The entrance end cap usually has a 2-3 mm hole drilled in it to allow ions to enter the ion trap, whereas the exit end cap usually has one to six 2-3 $\mathrm{mm}$ holes drilled in it to allow ions to exit the trap to the detector at the appropriate time. This system is called a quadrupole ion trap because the radially symmetrical electrodes create a quadrapolar field. The physical size of the trap is specified by the inscribed radius of the ring electrode and end-cap electrodes. The size of a QIT needs to contain dimensions that adhere to the relationship of

$$
r_{o}^{2}=2 z_{o}^{2}
$$

where $r_{o}$ is the radius of the ring electrode and $z_{o}$ is the distance from the center of the ring electrode to the end cap. This relationship needs to be maintained for the QIT to successfully control the ions contained in the trap in both the $\mathrm{r}$ (radial) and $\mathrm{z}$ (axial) directions. 


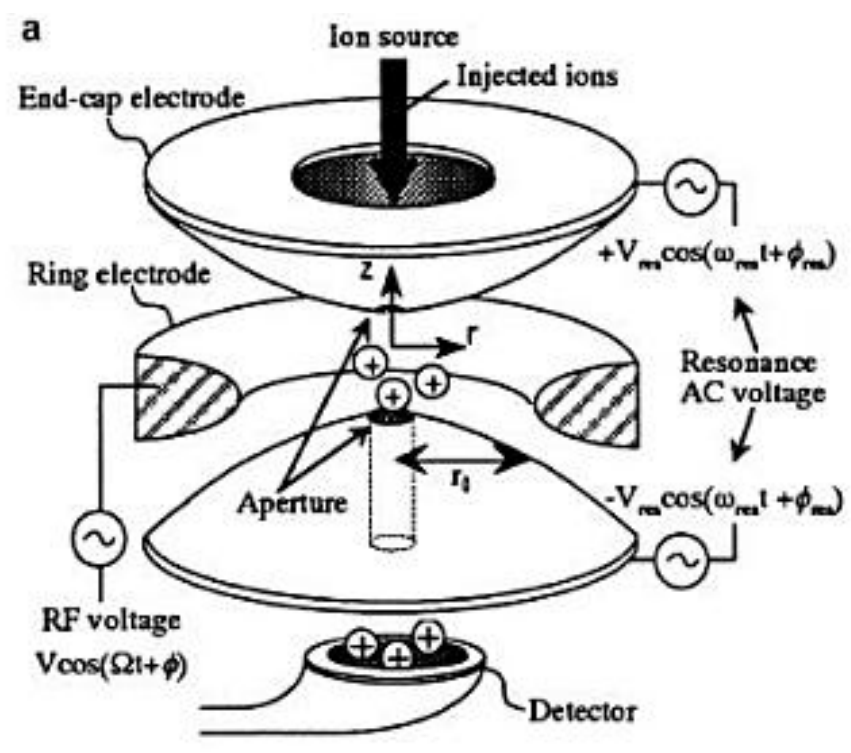

b

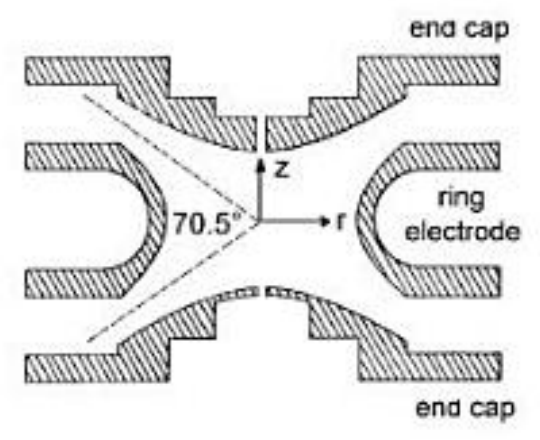

Figure 1.6. Illustration of a) how the ion trap functions during operation and b) a cross section of the ion trap. (Reprinted with permission from Gross, J. H., Mass Spectrometry: A Textbook..$^{\text {st }}$ ed.; Springer: Heidelberg, Germany, 2004.)

\subsubsection{Trapping the ions}

Ions are normally generated outside of the trap to prevent unwarranted ionmolecule reactions and to enable more flexibility in ion source optimization. External ion sources can also be exchanged or modified more easily, without affecting the performance of the trap. Common commercial sources include electrospray ionization $(\mathrm{ESI})^{112}$, Fast Atom Bombardment $(\mathrm{FAB})^{20}$, and matrix assisted laser desorption ionization (MALDI) ${ }^{113}$. After ionization, sample molecules are desolvated and focused using the ion optics, and introduced into the trap in a time-gated manner. The QIT then holds the ions inside the trap using an rf electric field on the ring electrode. The magnitude and frequency of the applied rf voltage will determine what mass to charge range will have a stable trajectory. Those ions that are stable within the trap are held and 
analyzed while those that are unstable collide with surfaces like the walls of the trap and are lost. In this way the voltage controlling these fields can be manipulated to specifically select those ions, which are desired for an experiment and reject those that are not.

In order for an ion to be held within the trap, it needs to be stable in both the $r$ and the $\mathrm{z}$ directions. The stability of an ion in a quadrapolar field has been described by Mathieu as

$$
\begin{aligned}
& a_{z}=\frac{-16 z U}{m\left(r_{o}{ }^{2}+2 z_{o}{ }^{2}\right) \Omega^{2}} \\
& q_{z}=\frac{8 z V}{m\left(r_{o}{ }^{2}+2 z_{o}{ }^{2}\right) \Omega^{2}}
\end{aligned}
$$

where $\mathrm{a}_{\mathrm{z}}$ and $\mathrm{q}_{\mathrm{z}}$ are the the stability coordinates in the $\mathrm{z}$-direction, $\mathrm{z}$ is the charge on the ion, $\mathrm{U}$ is the $\mathrm{DC}$ voltage, $\mathrm{V}$ is the $\mathrm{rf}$ amplitude, $\mathrm{m}$ is the mass of the ion, $\mathrm{r}_{\mathrm{o}}$ and $\mathrm{z}_{\mathrm{o}}$ are the dimensions of the ion trap and $\Omega$ is the frequency of the $\mathrm{rf}$ voltage. ${ }^{105}$ Most of the variables are constant in a typical experiment. The DC is usually zero volts and the $\Omega$ is usually fixed at $\sim 1 \mathrm{MHz}$, so the trap can only operate as a storage device with a variable low mass cutoff defined by the $\mathrm{rf}$ amplitude. Since all of the variables used to calculate $\mathrm{a}_{\mathrm{z}}$ are constant the stability of an ion in the ion trap can be considered using only $\mathrm{q}_{\mathrm{z}}$. An ion with a $\mathrm{q}_{\mathrm{z}}=0.908$ is said to be on the edge of stability. Ions with larger $m / z$ values and hence $\mathrm{q}_{\mathrm{z}}<0.908$ will be stable and ions with smaller $m / z$ values and $\mathrm{q}_{\mathrm{z}}>0.908$ will be unstable. When ions are held in the QIT they move in a Lissajous whose dimensions are 
defined by the secular frequency of the ions being trapped. The secular frequency of an ion in the $\mathrm{z}$ dimension is defined by the equation

$$
\omega_{z, n}=\left(n+\frac{1}{2} \beta_{z}\right) \Omega
$$

where $\omega$ is the secular frequency, $\mathrm{n}$ is the number of higher order frequencies, $\Omega$ is the angular frequency of the $\mathrm{rf},{ }^{105}$ and $\beta_{z}$, which is dependent on both $\mathrm{a}_{\mathrm{z}}$ and $\mathrm{q}_{\mathrm{z}} \cdot \beta_{\mathrm{z}}$ is approximated by the relationship

$$
\beta_{z}=\sqrt{a_{z}+\frac{1}{2} q_{z}^{2}}
$$

where $\beta_{z}$ needs to fall between 0 and 1 for the ion to be stable in the trap. ${ }^{114}$ We are only concerned with the secular frequency in the $\mathrm{z}$ direction because the trap is symmetrical. Because $\mathrm{a}_{\mathrm{z}}$ and $\mathrm{q}_{\mathrm{z}}$ define $\beta_{z}$, they tell us whether the secular motion of an ion is going to be stable inside the trap. The regions where an ion is going to be stable in the $\mathrm{r}$ and $\mathrm{z}$ directions can be illustrated visually using a Matheiu stability diagram (Figure 1.7). When ions are caught in the trap they are actually caught in an energy well created by the quadrupolar electric field. This energy well is characterized by the Dehmelt pseudopotiential well, which is defined as ${ }^{115}$ 


$$
D_{z}=q_{z} \frac{V}{8}=\frac{e V^{2}}{m\left(r_{o}{ }^{2}+2 z_{0}{ }^{2}\right) \Omega^{2}}
$$

This equation produces a voltage that defines how much energy it takes to get out of the well. From this equation we can see that one of the values that $D_{z}$ is dependent on is $q_{z}$ again showing that the stability of an ion will determine how efficiently it is held in the trap with less stable ions requiring less energy to escape the trap.

Two other important factors also need to be considered for the trap to function effectively. These include both the density and kinetic energy of the ions entering the trap. Because of the finite volume, Columbic repulsion between similarly charged ions will set a practical limit for the number of ions that can be simultaneously stored in the trap. If too many ions are allowed in the trap at one time, then this repulsion will cause the ion cloud to spread out from the center making it harder for the trap to control the ions. This space charge effect results in a loss in measurement accuracy and sensitivity. ${ }^{116}$ To prevent space charge the ion accumulation time must be regulated so that enough ions are allowed to enter the trap to produce good precursor ion signal without allowing so many ions in that space charge occurs. The optimum value for this accumulation time is normally determined experimentally in real-time and can vary depending on the source conditions. The other important factor to consider is the kinetic energy of the ions entering the trap. This is important because if the ions enter the trap with too much kinetic energy then they will travel straight through the trap without being affected at all. To prevent this from occurring the trap is normally filled with a helium bath gas. When the ions enter the trap they collide with the helium atoms and lose some of their kinetic 


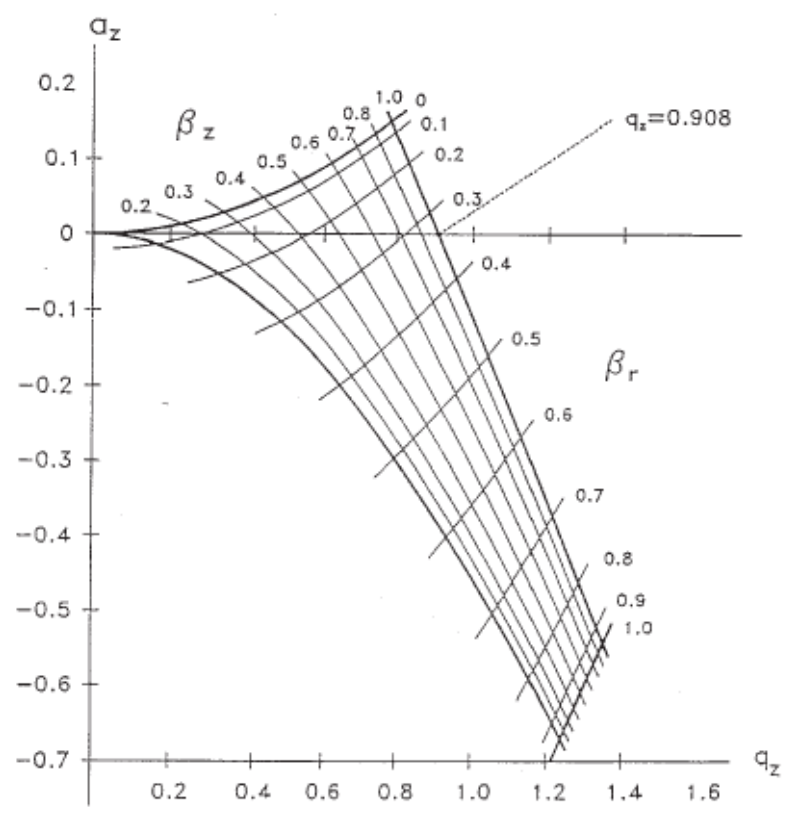

Figure 1.7. Matheiu stability diagram showing the $\mathrm{q}_{\mathrm{z}}$ values in which the ions are stable within the ion trap. In this case any ion with a $\mathrm{q}_{\mathrm{z}}$ value between 0 and 0.908 and a $\beta_{\mathrm{z}}$ value between 0 and 1 will be stable. (Reprinted with permission from March, R. E., Quadrupole ion traps. Mass Spectrom. Rev. 2009, 28 (6), 961-989.)

energy causing them to relax into the center of the trap. This reduction in kinetic energy through the use of bath gas is called collisional cooling and leads to a significant improvement in instrument sensitivity and resolution. ${ }^{114}$

\subsubsection{Ion manipulation}

Once ions have been captured in the QIT they can then be manipulated to perform experiments and ultimately to produce a mass spectrum. Ions can be manipulated in the trap using a process called resonant excitation. ${ }^{117,118}$ Resonant excitation occurs when a supplementary rf potential applied to the end cap electrodes matches the fundamental secular frequency of the ion in the $\mathrm{z}$ direction. ${ }^{114}$ The match between the supplemental or 'tickle' frequency and the motion of the ion causes the ion's kinetic energy to increase. If 
the amplitude of the applied potential is high enough the ion's kinetic energy level can exceed the depth of the pseudo potential well and the ion will be ejected from the trap. A QIT produces a mass spectrum through the use of the mass selective instability mode of operation. In this mode the rf voltage applied to the trap is increased over a period of time. As stated before in normal operation the endcaps of the trap are grounded. This means that $a_{z}$ is going to be 0 and the stability of the ions is only going to depend on $q_{z}$. Since we know that $\mathrm{q}_{\mathrm{z}}$ is dependent on the $\mathrm{AC}$ voltage when the rf voltage is increased the $\mathrm{q}_{\mathrm{z}}$ of the ions in the trap are going to increase as well. When the $\mathrm{q}_{\mathrm{z}}$ is forced above 0.908 or the $\beta_{z}=1$ limit in the stability diagram, all the ions of that mass will become unstable and be ejected from the trap. (Figure 1.7) Since the $\mathrm{q}_{\mathrm{z}}$ value of ions with a smaller $\mathrm{m} / \mathrm{z}$ are larger then that of those with a larger $\mathrm{m} / \mathrm{z}$, ions are ejected from the trap in order from the smallest $\mathrm{m} / \mathrm{z}$ to the largest $\mathrm{m} / \mathrm{z}$. When the ions are ejected they strike a detector, commonly an electron multiplier, which will record the ion current and transfer the signal to a computer where, via an amplifier and an analog to digital convertor, the signal will be converted into a mass spectrum.

The application of resonant frequencies can be used to manipulate the ions in other ways as well. For example, ions can isolated instead of ejected by applying a collection of frequencies to the trap all at once to eject ions of all unwanted $\mathrm{m} / \mathrm{z}$ values from the trap and leave behind the $m / z$ of interest. ${ }^{119}$ In this way, an experiment can focus on the products of one lone precursor ion without the complication of extraneous mass peaks being generated from other precursor ions. Resonant excitation can also be applied during ion fragmentation as well. ${ }^{120}$ In this method of fragmentation a resonant $\mathrm{rf}$ is applied to the trap in a smaller amplitude then would normally be applied for ion ejection. 
This causes the ions to gain energy without giving them enough to exceed the well depth. ${ }^{121}$ As the ions gain kinetic energy they collide with the helium bath gas present in the trap. Each collision with the bath gas allows the ion to convert some of its kinetic energy into internal energy and vice versa. When the internal energy of the ion exceeds the threshold for dissociation it will fragment. This type of fragmentation through collision is known as collision induced dissociation (CID). ${ }^{122}$

\subsection{Mass spectrometry techniques used to study lipids}

The ion trap is a versatile instrument that has been utilized by scientists to explore the role of lipids in plants and animals. One reason for the popularity of the ion trap is its ability to perform tandem MS experiments making this type of mass spectrometer compatible with a large variety of fragmentation techniques. Tandem MS is a process where the mass spectrometer can isolate and fragment an ion and its products multiple times within one experiment. These isolation and fragmentation steps are performed using the resonant excitation techniques described above. Ion traps are able to perform $\mathrm{MS}^{\mathrm{n}}$ experiments rather then just MS/MS experiments. ${ }^{18,123}$ This means that the ion trap can isolate and fragment the products of the original precursor almost indefinitely. The number of stages of MS that can be performed is normally limited by the ion signal. Each time a precursor is isolated, more of the ions in the trap are lost and eventually there aren't enough ions in the trap to observe a significant ion signal. $\mathrm{MS}^{\mathrm{n}}$ allows one to explore the structure of the product ions as they can be further trapped and fragmented too. This investigative power then allows the in-depth exploration of lipid structure and helps to determine their role in biological membranes, their role in diseases like obesity 
and Alzheimer's disease and their role in cellular functions like signaling and controlling what can partition in and out of the cell. ${ }^{124,125}$

Systems of nomenclature both for how to categorize overall lipid structures ${ }^{126,127}$ and for classifying fragment ions ${ }^{128-130}$ have been proposed for lipid analysis, but one specific system has not been universally adopted at this point. One major challenge with developing a system of nomenclature for the fragmentation of lipids is that the structure of different sub-classes of lipids can vary significantly from one another. The classification systems for these lipid fragments are similar to those developed for protein and peptide fragmentation in that bond fragments are denoted by specific letters depending on the bond position and the end of the lipid that retains the charge. For example, Figure 1.8 shows that the fragmentation of the lipid behind the hydroxyl group is considered a $\mathrm{P}$ fragment if the charge is retained on the lipid portion that does not contain the headgroup and a $\mathrm{G}$ fragment if the charge is retained on the portion of the lipid that does contain the headgroup.

\subsubsection{Collision induced dissociation (CID)}

Collision induced dissociation is one of the most common techniques used for studying biological compounds. As a target ion collides with helium atoms, some of its kinetic energy is converted to internal energy. Fragmentation occurs when this internal energy exceeds the strength of a set of bonds in the target molecule causing it to break apart. There are two different varieties of CID that are normally used to study biological compounds, low and high energy CID. The difference between the two is the amount of 

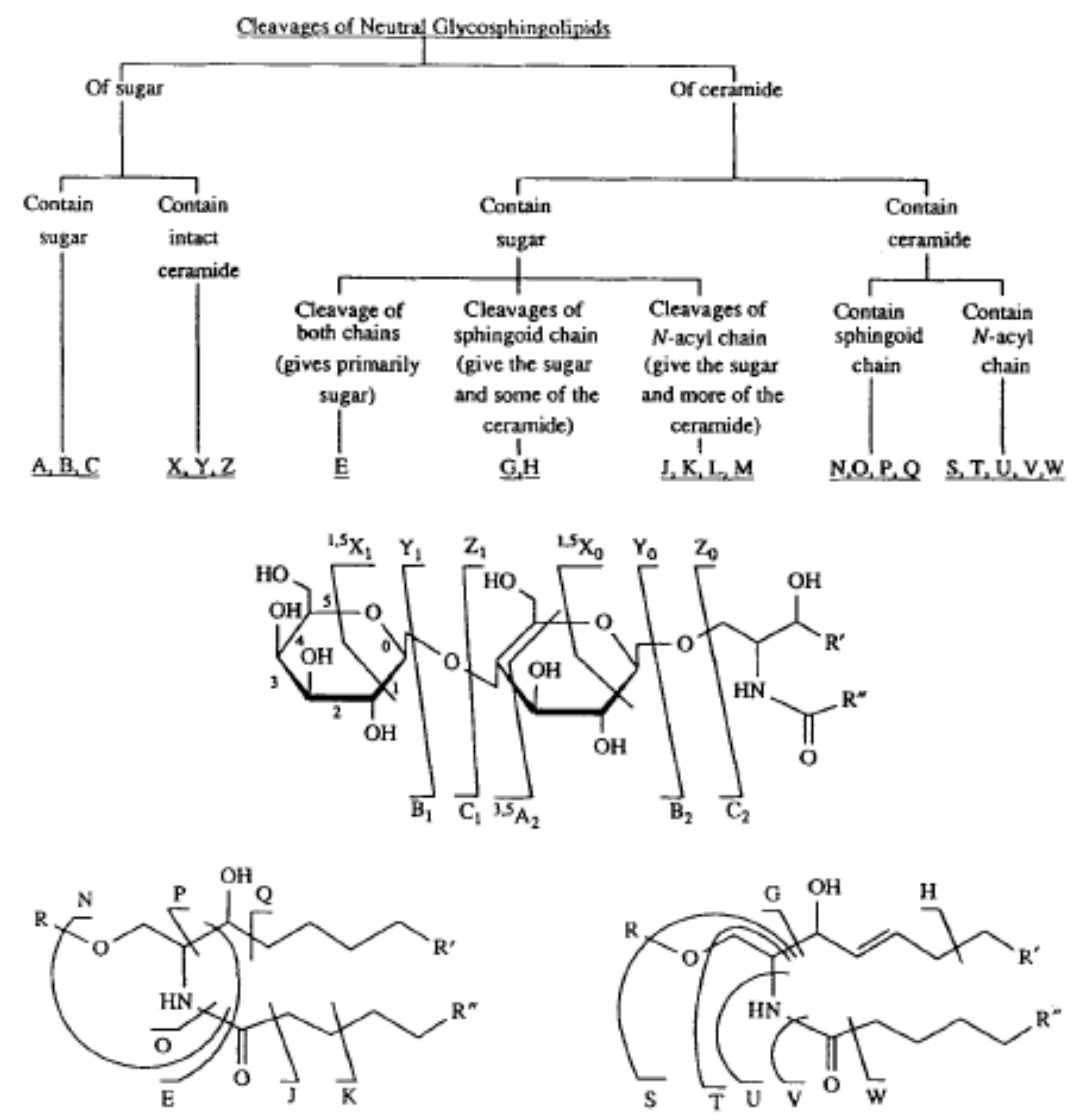

Figure 1.8. One example of a system of nomenclature for a specific subclass of lipids. This system was proposed by Adams et. al. for the classification of sphingolipids. (Reprinted with permission from Adams, J.; Ann, Q., Structure determination of sphingolipids by mass spectrometry. Mass Spectrom. Rev. 1993, 12 (1), 51-85.)

kinetic energy made available to the target compound and the number of collisions that occur before fragmentation. Low-energy CID is considered to be a slow heating technique ${ }^{131}$ and typically uses lab energies ranging from $0-100 \mathrm{eV}$ and requires tens to hundreds of collisions before fragmentation occurs. High energy CID on the other hand utilizes energies above $1 \mathrm{keV}$ (lab frame) and normally occurs in around five collisions or less. ${ }^{122,132}$ High and low-energy CID will produce different fragment types and relative abundances due to the difference in energy imparted during fragmentation. Low-energy CID will typically produce more efficient fragmentation than high energy CID but will 
not preserve the more labile groups like post-translational modifications of peptides. $^{11}$ High-energy CID typically produces extensive fragmentation because it accesses higherenergy fragmentation pathways that are not accessible with low-energy CID.

The majority of the CID experiments performed on lipids are done using lowenergy CID. This popularity is due to the fact that low-energy CID capability has become standard issue for many mass spectrometry systems making it widely available in many laboratories. Low-energy CID has been used to study a wide variety of lipid types ranging from single fatty acid chains to glycerophospholipids. ${ }^{133}$ Because of the variety of lipid subclasses it is often prudent for a research project to focus specifically on one or two types of lipid. One area that has received considerable attention using low-energy CID is the study of glycerophospholids and phosphosphingolipids. Glycerophospholids have been studied using low-energy CID in both the positive and negative mode. Upon fragmentation these lipids normally produce a variety of products the most common of which include, the loss of a fatty acid chain, fragmentation within the fatty acid chain and loss of the lipid headgroup. ${ }^{33,70,71}$ (Figure 1.9) During analysis, it can sometimes be challenging to obtain a large ion signal for lipids that do not protonate or deprotonate easily. These lipids can usually be ionized more effectively through the addition of adducting metal ions in the solution. Metal adducts commonly include $\mathrm{Na}$, $\mathrm{Li}$, and $\mathrm{K}$, but a variety of other metals like silver have been tested as well. ${ }^{134}$ Fragmentation of lipid/metal adduct ions normally produces the loss of the fatty acid chains and loss of the headgroup similar to the protonated form. The metal adduct also causes the loss of the trimethylamine from the headgroup and the loss of $\mathrm{O}, \mathrm{O}$ '-dimethylenephosphoric acid, which is usually not observed from the protonated precursor. ${ }^{71}$ Low-energy CID has also 


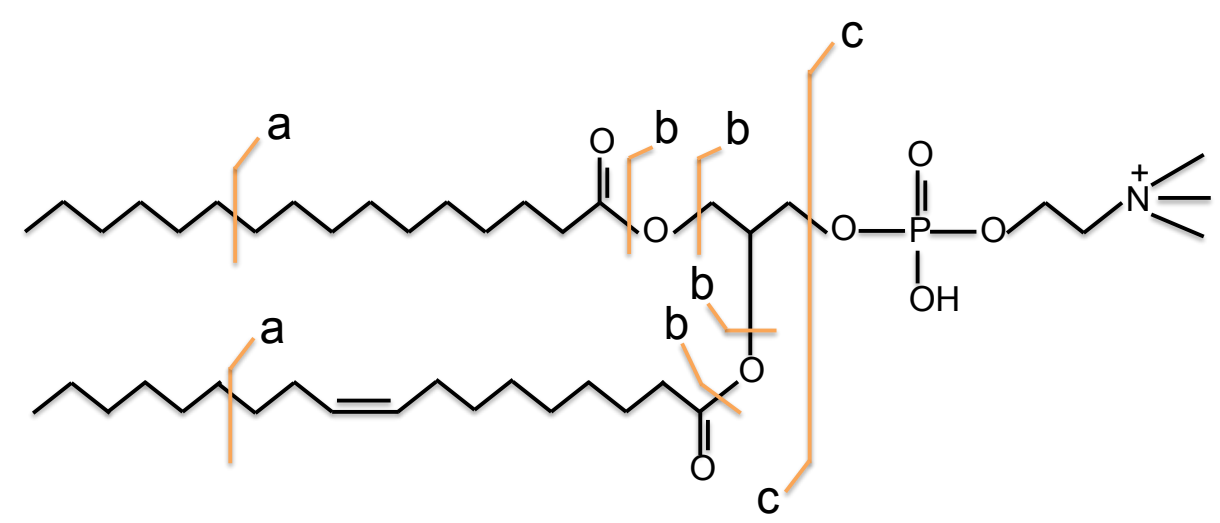

Figure 1.9. An example of a glycerophospholipid labeled with the common fragments that occur during CID. a)=fatty acid fragments, $b$ )=the loss of an entire fatty acid chain, and $\mathrm{c}$ )=the loss of the phosphocholine headgroup.

been used to explore the location of double bonds in lipids, either through the use of external compounds like ozone that react specifically with double bonds causing unique fragmentation ${ }^{135-137}$ or through multi-stage mass spectrometry $\left(\mathrm{MS}^{\mathrm{n}}\right) .{ }^{72}$

High-energy CID is another fragmentation technique that is used to interrogate lipids. High-energy CID is normally performed using a magnetic sector or TOF instrument, like a matrix assisted laser desorption ionization-time of flight (MALDITOF/TOF). ${ }^{122} \mathrm{~A}$ TOF/TOF system transfers ions from the ionization source and accelerates them down the drift tube using an electric field set around $20 \mathrm{kV}$, which grants the ions approximately $20 \mathrm{keV}$ of kinetic energy. ${ }^{138} \mathrm{The}$ ions are then directed to a collision cell in between the two TOF segments where some of this energy is converted to internal energy via collisions with helium or argon gas atoms. ${ }^{139}$ Thus, it is much easier to perform high energy CID with a TOF instrument that achieves the necessary energy as a natural part of its analysis then it is to try and achieve this level of energy through another means, like resonant excitation in an ion trap. However, the cost of 
purchasing systems like a TOF/TOF is often prohibitive. Despite the expense there have been several groups that have implemented high-energy CID to study lipids like fatty acids, ${ }^{140}$ glycerophospholipids, ${ }^{42,44}$ and triacylglycerols. ${ }^{43,130}$ Unlike low-energy CID, when Shimma et. al. performed high energy CID experiments on glycerophosphocholines and galactosylceramides they found that they could produce multiple fragments at different points along the fatty acid chain in addition to the commonly observed headgroup. ${ }^{42}$ They showed that high-energy CID was effective in positive and negative mode and that effective fragmentation could be produced directly from mouse brain tissue sections. Cheng and Gross used high-energy CID to study triacylglycerols, which also showed extensive fragmentation along the fatty acid chains. ${ }^{141}$ They found that the fragmentation pattern produced by the $\left[\mathrm{M}+\mathrm{NH}_{4}\right]^{+}$precursor ion was informative in determining the degree of unsaturation and carbon length of the fatty acid chain, and also for deciphering the position and number of double bonds on the fatty acid chains too. Fragmentation within the fatty acid chains of lipids occurs through a process called charge remote fragmentation $(\mathrm{CRF}) .{ }^{141-143} \mathrm{~A}$ CRF reaction is characterized as one that has no interaction with any of the charges on the molecule and thus can occur regardless of the charge state. ${ }^{141} \mathrm{CRF}$ is a favored fragmentation pathway for high energy CID and is normally observed as sequential losses of $\mathrm{C}_{n} \mathrm{H}_{2 n+1}$ or $\mathrm{C}_{n} \mathrm{H}_{2 n+2}$ fragments from the target precursor. Mechanisms for how CRF occurs include a 1,4 elimination reaction ${ }^{141}$ or a homolytic carbon-carbon bond cleavage. ${ }^{142}$ Further experimentation is still being performed to examine this process as one mechanism has yet to explain all of the types of experimental fragmentation observed during this process. 
Although both low-energy and high-energy CID have been effective for studying different aspects of lipids they also have disadvantages that need to be considered as well. One disadvantage associated with the use of CID is that it is an ergodic technique, which means that the energy applied to the lipid molecule is distributed throughout the molecule before fragmentation occurs. In this way, the weakest bonds within the molecule are normally broken first. The ergodic nature of this process can be a disadvantage in lowenergy CID if the area of interest in the lipid is not one of the weakest bonds in the molecule. This ergodic nature can also be a problem because the two or three bonds that are most easily broken usually dominate the spectrum. For example, when Hsu and Turk performed low-energy CID on the protonated form of 16:0/18:1 PC and 16:0/16:0 PC, they found that the mass spectra contained just three or four peaks. ${ }^{71}$ These spectra contained peaks corresponding to the headgroup and the complete loss of the fatty acid chains. Although these peaks do give valuable information on the type of lipid being examined, and the length of the fatty acid chains, they do not give other information like the composition of the fatty acid chain or the headgroup. If this information was a point of interest then it would be necessary to find another way to ionize the compound (i.e. the use of a metal adduct) or through the use of another fragmentation technique. High energy CID tends to have to opposite problem from low-energy CID. Instead of having a spectrum that may only have a few peaks you may instead have a spectrum with far too many. High energy CID has the capability to break up the lipid at many different positions, which in some cases can lead to spectra that are complex and more challenging to interpret. Also, because high energy CID is generally performed in TOF instruments it 
is also necessary to consider post source decay ions, which may interfere with the analysis. ${ }^{144}$

\subsubsection{Post source decay (PSD)}

Another fragmentation technique used alongside CID for the study of biological materials is post source decay (PSD). Post source decay is a technique performed using MALDI-TOF instruments. In a TOF instrument the ions collected from the MALDI source are accelerated across two metal plates. The potential is used to accelerate the ions to a specific kinetic energy. PSD occurs when ions decay within the flight tube of the mass spectrometer after the precursor ion has been accelerated. ${ }^{145}$ PSD products cannot be identified in a linear TOF mass spectrometer because PSD products have the same kinetic energy as the intact precursor, so the precursor and its PSD products reach the detector at the same time. To observe PSD products, an ion mirror or a reflectron (Figure $1.10)$ is necessary. An ion mirror generates an electric field across several plates in front of the in-line TOF detector. As the ions enter the detector they will penetrate the field to different degrees depending on the velocity and the mass to charge ratio of the incoming ions. Larger ions will penetrate the field more deeply then smaller ions. The difference in momentum will cause ions with a larger $\mathrm{m} / \mathrm{z}$ to take a longer time to be reversed by the electric field, thereby separating them in space from the ions with a smaller $m / z$ that are turned around more quickly. This process allows two ions that arrive with the same velocity (as from PSD) to be separated according to their momentum, which can be related to $m / z$. In a regular MALDI-TOF instrument, PSD products are considered to be a nuisance because they give a false impression of the intensity of a given precursor ion. Since the precursor ion and its PSD products reach the detector at the same time they 


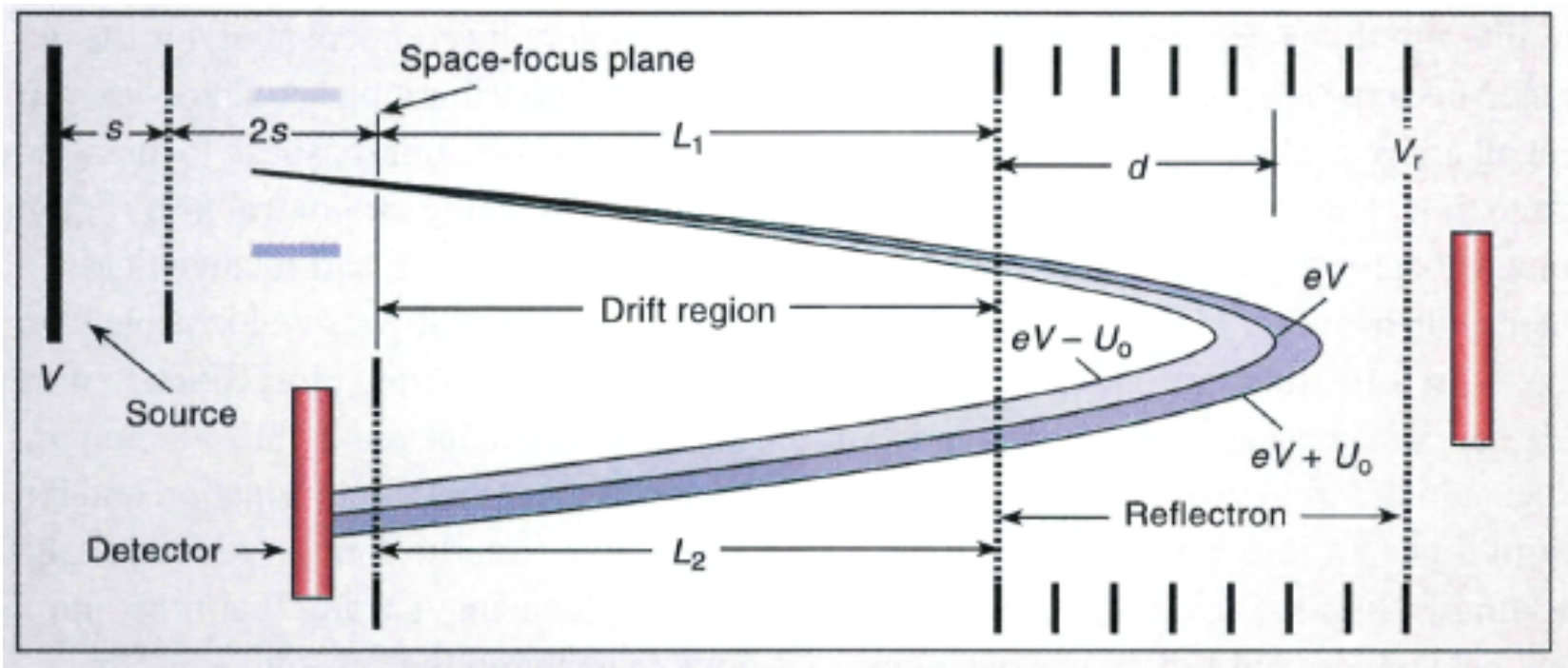

Figure 1.10. Schematic of a typical reflectron unit in a time-of-flight mass spectrometer where $L_{1}$ and $L_{2}$ are length of the drift regions, $e V$ is the kinetic energy of the ions, $U_{0}$ is the kinetic energy distribution of ions of the same type, $s$ is the distance traveled within the source, $\mathrm{d}$ is the distance travelled into the reflectron, $\mathrm{V}$ is the voltage used to accelerate the ions from the source and $\mathrm{V}_{\mathrm{r}}$ is the voltage applied to the reflectron. (Reprinted with permission from Cotter, Robert J., The new time-of-flight mass spectrometry. Anal. Chem. 1999, 71 (13), 445A-451A. Copyright 2014 American Chemical Society.)

appear as one peak in the mass spectra, even if a substantial fraction actually decays during the flight. Another reason that PSD products are considered a nuisance in MALDI-TOF is that they can cause peak broadening. When an ion undergoes PSD in the flight tube its velocity or kinetic energy will change slightly during fragmentation in accordance with conservation of momentum causing the resulting spectral peak to be wider then expected. ${ }^{146}$ In MALDI-TOF/TOF, PSD products can make it difficult to determine which products were achieved from PSD and which originate from CID. ${ }^{144}$ For example, in a TOF/TOF experiment the precursor ion and its PSD products would all be isolated by the ion gate and allowed into the collision cell at the same time. The products released into the second drift tube after fragmentation would be primary and secondary 
products of the precursor. Because of this potential complication some systems have been developed to try and remove or reduce PSD products from the experiment. ${ }^{144}$ Despite these disadvantages PSD has also come to be recognized as viable fragmentation method in its own right. When used as the primary method of fragmentation, PSD can produce fragmentation patterns that are unique when compared to or combined with other methods like CID. ${ }^{147,}{ }^{148}$ For example, Zhang et. al. found low-energy CID and PSD to be complementary techniques. Low-energy CID of a multiply charged peptide excelled at the fragmentation of the basic sites of the peptide and the production of multiply charged fragments whereas PSD of a singly charged peptide provided a more comprehensive $-\mathrm{b}$ and $-\mathrm{y}$ ion series down the peptide backbone. ${ }^{147}$ PSD has been applied for the study of a variety of biomolecules including peptides ${ }^{146,149,150}$ and lipids. ${ }^{151-154}$

\subsubsection{Fragmentation with potential energy}

Another effective way to fragment target ions is through the use of potential energy. In this form of fragmentation an electron is typically taken from or donated to the target ion, which induces a secondary reaction within that compound that causes fragmentation. This reaction is normally instigated through interaction between the target ion and another ion, molecule or electron. Examples of techniques that utilize this form of fragmentation are electron capture dissociation (ECD) and electron transfer dissociation (ETD).

Electron capture dissociation was first developed by McLafferty and co-workers in 1998. ${ }^{1,4}$ This type of fragmentation is normally performed in an FT-ICR mass spectrometer because this kind of instrument can trap both the target precursor and the low-energy electrons in its collision cell facilitating the reaction. ${ }^{155} \mathrm{ECD}$ functions by 
exposing a multiply-charged target cation with low-energy electrons $(<0.2 \mathrm{eV}) \cdot{ }^{156} \mathrm{The}$ target ion then accepts one of these electrons, thereby generating a hydride radical within the compound that is then free to migrate and cause fragmentation. Electron capture dissociation is most commonly associated with the investigation of peptides and proteins where it produces $\mathrm{c} / \mathrm{z}$ type fragments that are complementary to the $\mathrm{b} / \mathrm{y}$ type fragments that are normally associated with CID. ${ }^{3}$ However, because the addition of an electron causes a decrease in the charge state ECD is not applicable to singly-charged precursors. Currently, ECD has not been extensively used for lipid analysis.

Electron transfer dissociation was first developed by Hunt and co-workers in 2004. ${ }^{5}$ ETD functions by introducing the target ion to an electron-rich molecule like fluoranthene or anthracene which then donates an electron to the target molecule. Just like in ECD this radical electron is then free to migrate around the target molecule and cause fragmentation. Fragmentation then normally results in the production of radical $\mathrm{c} / \mathrm{z}$ type ions. ${ }^{7,8}$ The major advantage of ETD versus ECD is that ETD is more accessible. ETD can be performed in cheaper ion trap instruments whereas ECD is normally associated with the more expensive FT-ICR mass spectrometers. ${ }^{157-160}$ Because ETD also decreases the charge state of the target molecule it has the same limitation as ECD in that it is not applicable to singly charged precursors. ETD has mainly been used to study peptides although there have been a few reports where it has also been used to study lipids like sodiated glycerophosphocholines. ${ }^{161}$

\subsection{Metastable atom-activated dissociation (MAD)}

Zubarev and coworkers were the first to demonstrate the use of metastable atoms to dissociate charged ions in an ion trap. ${ }^{162}$ They termed the technique metastable induced 
dissociation of ions (MIDI), and the technique was applied to both positive and negative ions. This experiment was followed by reports from the groups of Berkout and Jackson in 2006 and 2009, respectively. ${ }^{9} 163$ Berkout's group was able to show that MIDI was effective in fragmenting singly charged as well as multiply charged peptides. ${ }^{146}$ The Jackson group termed the technique metastable atom-activated dissociation (MAD) and showed that isobaric side chains like leucine and isoleucine could be distinguished, and that proline residues could also be cleaved across the ring. ${ }^{9,} 164$

A metastable atom is usually a noble gas atom that has an electron in a prolonged excited state. In the case of a helium metastable atom source such as a plasma or a fast atom bombardment (FAB) gun, the electron is promoted into the low-lying $2^{1} \mathrm{~S}_{0}$ or the $2^{3} \mathrm{~S}_{1}$ states through a variety of mechanisms such as electron excitation, electron transfer and electron capture. If the electron is promoted into the singlet $\mathrm{S}_{0}$ state then it is spin antiparallel to the ground state and the helium atom is in a parahelium energy level. If the electron is promoted into a triplet $S_{1}$ state then it is spin parallel with the ground state and is in an orthohelium energy level. The orthohelium energy levels are lower in energy then the parahelim energy levels due to their higher spin multiplicity according to Hund's rule. ${ }^{165}$

Normally, an excited electron will relax by spontaneous emission and return to the ground state after a certain period of time. However, relaxation from the $2^{1} \mathrm{~S}_{0}$ and $2^{3} \mathrm{~S}_{1}$ states of helium does not occur as quickly because these states are spin forbidden through quantum mechanical spin rules. Transition to the $2^{1} \mathrm{~S}_{0}$ state of helium is forbidden because only $\mathrm{J} \neq 0$ transitions are allowed. Transition to the $2^{3} \mathrm{~S}_{1}$ state of helium is 
forbidden because it induces a spin flip making its $\mathrm{m}_{\mathrm{s}}$ quantum number temporarily incompatible. An example of an energy level diagram for helium is shown in

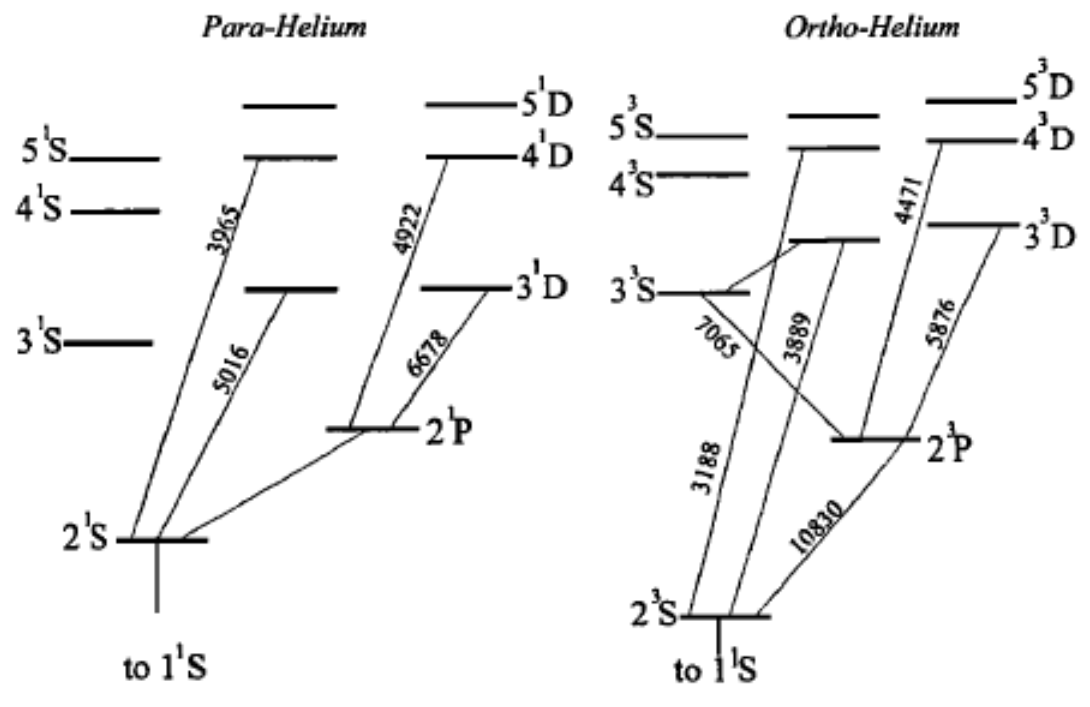

Figure 1.11. The molecular energy level diagram of a helium atom showing emission lines that populate the metastable atom levels. (Reprinted with permission from Noto, J.; Kerr, R.; Shea, E.; Waldrop, L.; Fisher, G.; Rudy, R.; Hecht, J.; Gonzalez, S.; Sulzer, M.; Garcia, R., Evidence for recombination as a significant source of metastable helium. $J$. Geophys. Res.-Space 1998, 103 (A6), 11595-11603.) The $1^{1} \mathrm{~S}$ to $2^{1} \mathrm{~S}$ transition of parahelium and the $1^{1} \mathrm{~S}$ to $2^{3} \mathrm{~S}$ transition of ortho-helium are quantum forbidden and result in elevated lifetimes (metastability).

Figure $1.11 .{ }^{167}$ The fragmentation power of a metastable atom lies in its potential energy or the metastable energy state. The metastable atom states lie at $20.6 \mathrm{eV}$ and $19.8 \mathrm{eV}$ above the ground state for the $2^{1} \mathrm{~S}_{0}$ and $2^{3} \mathrm{~S}_{1}$ states respectively. A table of the characteristics of different metastable gas atoms including helium is shown in Table $1.1 .^{166}$

From Table 1.1 we can see that the excitation energy can range from a minimum of $8.32 \mathrm{eV}$ for xenon to a maximum of $20.62 \mathrm{eV}$ for helium. This energy range allows a MAD operator to have some control of the energetics delivered to the target compound, 
which could affect the degree or type of fragmentation observed. Because these excited states last for an extended period of time, $7900 \mathrm{~s}$ for $2^{3} \mathrm{~S}_{1}$, a metastable atom can survive the journey from the metastable source to the target ions in the trap. The prolonged travel Table 1.1. Characteristics of Metastable Atoms generated from Noble Gases ${ }^{a}$

\begin{tabular}{cccccc}
\hline Atom & State & $\begin{array}{c}\text { Excitation } \\
\text { Energy }(\mathrm{eV})\end{array}$ & $\begin{array}{c}\text { Ionization } \\
\text { Potential }(\mathrm{eV})\end{array}$ & Lifetime $^{\mathrm{b}}(\mathrm{s})$ & Polarizability $\left(\AA^{3}\right)$ \\
\hline $\mathrm{He}$ & ${ }^{2} \mathrm{~S}_{0}$ & 20.62 & 3.97 & $\sim 0.02$ & $>9$ \\
& ${ }^{3} \mathrm{~S}_{1}$ & 19.82 & 4.77 & $>7900$ & 46.9 \\
\hline $\mathrm{Ne}$ & ${ }^{3} \mathrm{P}_{0}$ & 16.72 & 4.85 & 430 & \\
& ${ }^{3} \mathrm{P}_{2}$ & 16.62 & 4.95 & 24.4 & 27.8 \\
\hline $\mathrm{Ar}$ & ${ }^{3} \mathrm{P}_{0}$ & 11.72 & 4.04 & 44.9 & \\
& ${ }^{3} \mathrm{P}_{2}$ & 11.55 & 4.21 & 55.9 & 47.9 \\
\hline $\mathrm{Kr}$ & ${ }^{3} \mathrm{P}_{0}$ & 10.56 & 3.43 & 0.49 & \\
& ${ }^{3} \mathrm{P}_{2}$ & 9.92 & 4.08 & 85 & 50.7 \\
\hline $\mathrm{Xe}$ & ${ }^{3} \mathrm{P}_{0}$ & 9.45 & 2.68 & 0.08 & \\
& ${ }^{3} \mathrm{P}_{2}$ & 8.32 & 3.81 & 150 & 63.6 \\
\hline
\end{tabular}

$a$ - adapted from a table by P. E. Siska ${ }^{166}$ in Molecular-beam studies of Penning ionization Rev. Mod. Phys. 1993, 65, 337-412. (Reprinted in part with permission from Siska, P.E. Molecular-beam studies of Penning ionization Rev. Mod. Phys. 1993, 65, 337-412. Copyright 2013 by the American Physical Society)

$b$ - in the absence of collisions

time however, assumes that the metastable atoms do not experience collisions on their path to the target analyte. When a metastable atom $\left(\mathrm{A}^{\mathrm{M}}\right)$ undergoes a collision with an atom, molecule, surface or ion it can undergo several different reactions ranging from kinetic energy transfer (Eq. 7), ionization of the target (Eq. 8), excitation of the target $\left(\mathrm{B}^{* *}\right)\left(\right.$ Eq. 9), ${ }^{168}$ and a combination of ionization and excitation (Eq. 10).

$$
A_{\text {fast }}{ }^{M}+B_{\text {slow }} \rightarrow A_{\text {slow }}{ }^{M}+B_{\text {fast }}
$$




$$
A^{M}+B \rightarrow A+B^{+}+e^{-}
$$

$$
A^{M}+B \rightarrow A+B^{* *}
$$

$$
A^{M}+B \rightarrow A+B^{+*}
$$

In our MAD experiments, metastable atoms are generated using an Ion Tech saddle field FAB gun. A high voltage is applied to the anode, which is located between two grounded cathode rings. (Figure 1.12) An electric discharge formed between the anode and the cathodes ionize some of the gas atoms in the FAB gun igniting a plasma.

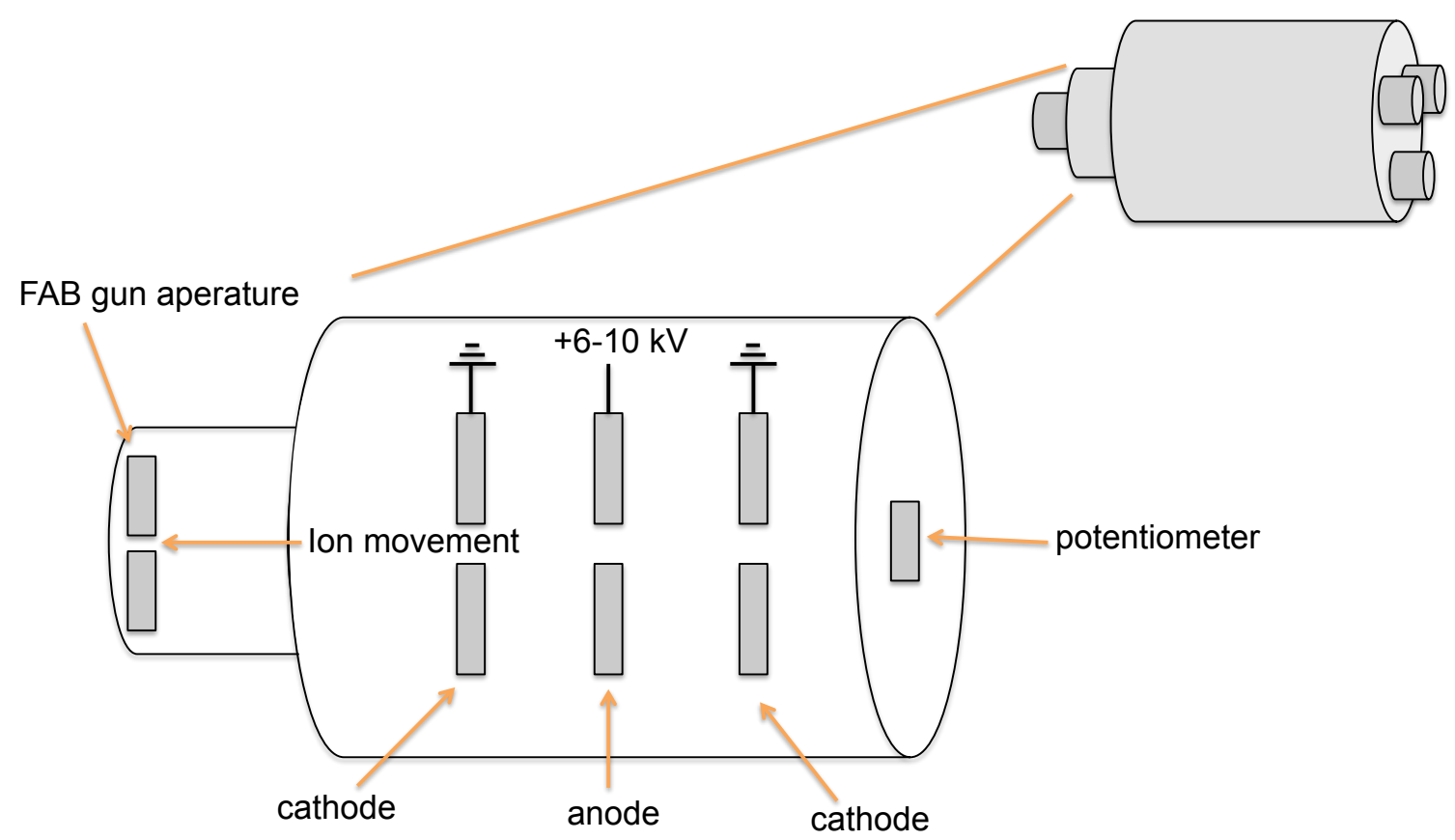

Figure 1.12. Schematic of the position of the cathodes and anode within the end of the FAB gun. 
The electrostatic fields created during this process cause the electrons to move in an oscillatory path sustaining the plasma. ${ }^{162}$ The plasma can generate several products from the FAB gas including electrons and fast moving ions. Ions generated by the high-energy electrons in the plasma are then accelerated towards the cathodes. Ions that pass through the top cathode are collected on a monitoring electrode that is connected to a potentiometer. Ions that pass through the bottom cathode interact with a region of neutral gas atoms of the same type and undergo charge exchange becoming neutral fast-ions. Charge exchange can occur without significant loss in momentum, thereby allowing the neutralized metastable atoms to leave the gun with approximately the same energy and direction as the ions. ${ }^{20}$ (Eq. 11)

$$
A^{+\bullet}{ }_{(\text {fast })}+A^{0} \rightarrow A_{(\text {fast })}^{M}+A^{+\bullet}
$$

The metastable atoms then leave the gun through a $1 \mathrm{~mm}$ orifice, along with any remaining fast ions and electrons generated during the process. In this way the FAB gun can create a concentrated beam of ions with a predictable direction. ${ }^{162}$ Unlike Zubarev's group, we normally remove excess ions and electrons using a pair of off-axis deflection electrodes, which are mounted to the end of the FAB gun.

When the metastable atoms enter the trap and come into close proximity to the target ions they can induce fragmentation through two main processes including Penning ionization and electron transfer. The first prominent fragmentation process that occurs in MAD is Penning ionization. Penning ionization was first proposed in 1927 by F.M. Penning and is shown in equation $8 .{ }^{169,170}$ He discovered this reaction while studying the 
premature breakdown of argon and neon gas when gas impurities were introduced into the system. ${ }^{166,170}$ Penning ionization of cations is shown in equation $12 .{ }^{164,}, 169$

$$
A^{M}+B^{n+} \rightarrow A^{0}+B^{(n+1)+} \cdot+e^{-}
$$

Interestingly, spin conservation rules require that the free electron come from the metastable atom and not from the ion. The electron from the precursor ion ends up in the ground state of the interacting rare gas atom causing the ejection of its excited electron and a return to the ground state for the rare gas atom. The radical generated on the target compound by the metastable atom is then free to migrate within the analyte and cause fragmentation. For Penning ionization to occur the metastable atom needs to be close enough to the target ion so that the $S^{1}$ orbital of the metastable atom can overlap with whatever atom the metastable atom is abstracting the electron from. In this way, metastable atoms can only Penning ionize things that they can get close to making the confirmation of the target ion an important factor.

The second way that MAD can cause fragmentation of a target analyte is through electron transfer. The Zubarev group found that some of the products they achieved after the MIDI of peptide cations looked similar to the products of ECD or ETD. They determined that the metastable atoms were able to donate an electron to the target compound and cause a decrease in charge state. This reaction was initiated through the use of an ion-pair collision complex and preceded through the reaction shown below. (Eq. 13) ${ }^{162}$

$$
A^{M}+B^{n+} \rightarrow A^{+\bullet}+B^{(n-1)+\bullet}
$$


The radical product ion then undergoes dissociation through radical migration throughout the compound. The competition between this electron transfer reaction (Eq. 13) and the Penning ionization (Eq. 8) can result in the appearance of both an oxidized or Penning ionized peak and a reduced or electron transfer peak. Charge reduction is most commonly observed for high mass multiply charged species. Penning ionization is more commonly observed for $1+$ or multiply charged peptides. ${ }^{9,10}$

MAD has been accomplished in both QIT and LIT mass spectrometers. In the QIT the metastable atoms enter the trap through a $2 \mathrm{~mm}$ hole drilled in the ring electrode. ${ }^{9,} 162$ In a linear ion trap mass spectrometer the metastable atoms were introduced radially into the collision cell. After fragmentation the products from the reaction of the metastable atoms and the target analytes were carried into the TOF for separation and identification. ${ }^{163,164,171}$ Rather then relying on a FAB gun to generate metastable ions in their LIT-TOF instrument the Berkout group utilized a glow discharge source that allowed metastable atoms to flow into the system through a conduction limit.

Although metastable atoms are neutral at long range, they are extremely polarized and behave like positive ions at molecular distances. This is because although they are formally neutral, metastable atoms are attracted to areas of the target molecule that have high electron densities and tend to abstract electrons. Metastable atoms also have large collision cross sections and are attracted to regions of high electron density like $\pi$ orbitals and lone pairs of electrons. Many of these characteristics of metastable atom behavior have been determined using metastable induced spectroscopy (MIES) and penning ionized electron spectroscopy (PIES). ${ }^{172-176}$ 
The propensity for PI to occur is reflected in the large number of background ions observed below $\mathrm{m} / \mathrm{z} 200$, even though there are no reagents deliberately added. The presence of this background signal can make it difficult to identify fragments with $\mathrm{m} / \mathrm{z}<$ 200 that do not have large signal intensities. Despite this limitation MAD also has a set of unique advantages that make it appealing as a fragmentation technique. Firstly, MAD has the ability to fragment singly charged precursor ions that are inaccessible to other techniques like ECD and ETD. Secondly, MAD has the ability to cause an ETD-like electron transfer, so it can also decrease the charge of singly charged negative ions. This gives MAD the versatility to examine both positive and negative ions. Another advantage of MAD is in its ability to produce unique fragmentation patterns compared to other current fragmentation techniques. For example, the Jackson group demonstrated that for $2+$ and $3+$ tau peptides that MAD could produce up to 17 unique fragments for the doubly charged peptides and up to 16 unique fragments for the triply charged peptides when compared to CID and ETD. MAD was also shown to produce an average sequence coverage of $83 \%$ for doubly charged peptides and $91 \%$ with triply charged peptides compared to the $67 \%$ and $71 \%$ for CID and $62 \%$ and $73 \%$ for ETD. ${ }^{10}$ Until now MAD has mainly been applied to the study of peptides. This work extends the applications to lipids as well. 


\section{CHAPTER 2 : INSTRUMENT OPTIMIZATION FOR PRODUCTION OF OPTIMUM RESPONSE DURING MAD ANALYSIS}

\subsection{Introduction}

The MAD system used in this project was constructed by Dr. Jackson and previous group members in a Bruker Esquire LC mass spectrometer. After extensive use as an experimental system, this instrument began to falter in performance and became unreliable. This unreliability manifested in different ways including issues ranging from software errors to the inability to accurately reproduce prior experiments. Extensive effort was required to bring the instrument back to a satisfactory level of performance. The first portion of this project therefore focuses exclusively on the troubleshooting and then optimization of the instrument.

\subsection{Results and Discussion}

\subsubsection{Instrument assessment and course of action}

Over the course of several experiments in September 2011, it was determined that the instrument was not working properly. Instrument malfunction was being routinely demonstrated in both the hardware and software of the instrument in several ways. One indication of this decrease in system performance was an immediate loss of signal from the mass spectrometer when the FAB gun was turned on. Restarting the instrument and its associated computer could restore signal but the regained ion signal would only be retained until the $\mathrm{FAB}$ gun was turned on once again. This loss of signal was thought to 
be associated with an overcurrent on the ring electrode caused by a large number of metastable atoms striking the ring electrode and aside from causing signal loss would also generate a number of software errors as well. This charging effect did not happen during every analysis but it did occur consistently enough that it was a potential problem during any experiment.

a)

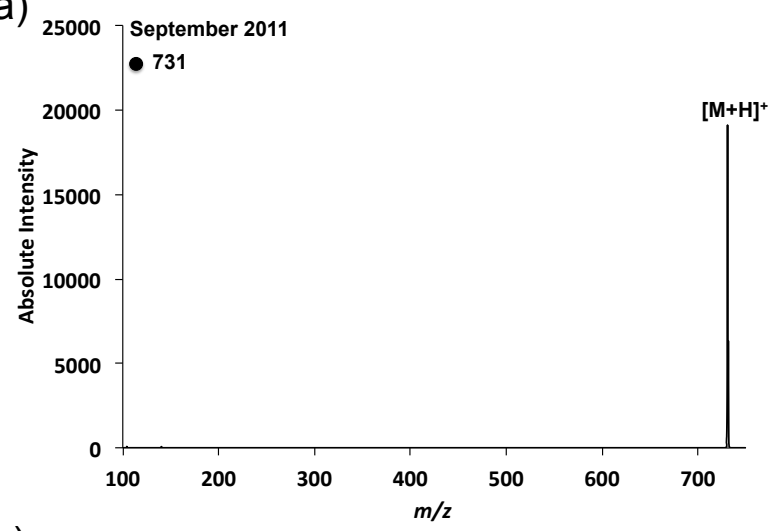

c)

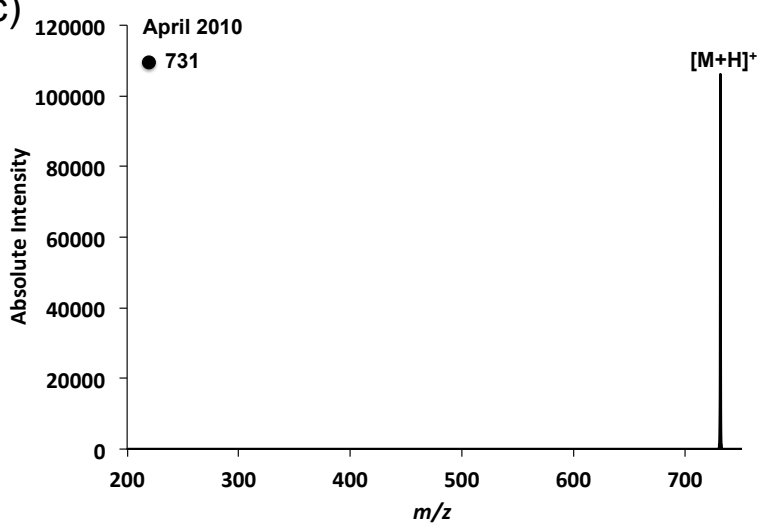

b)
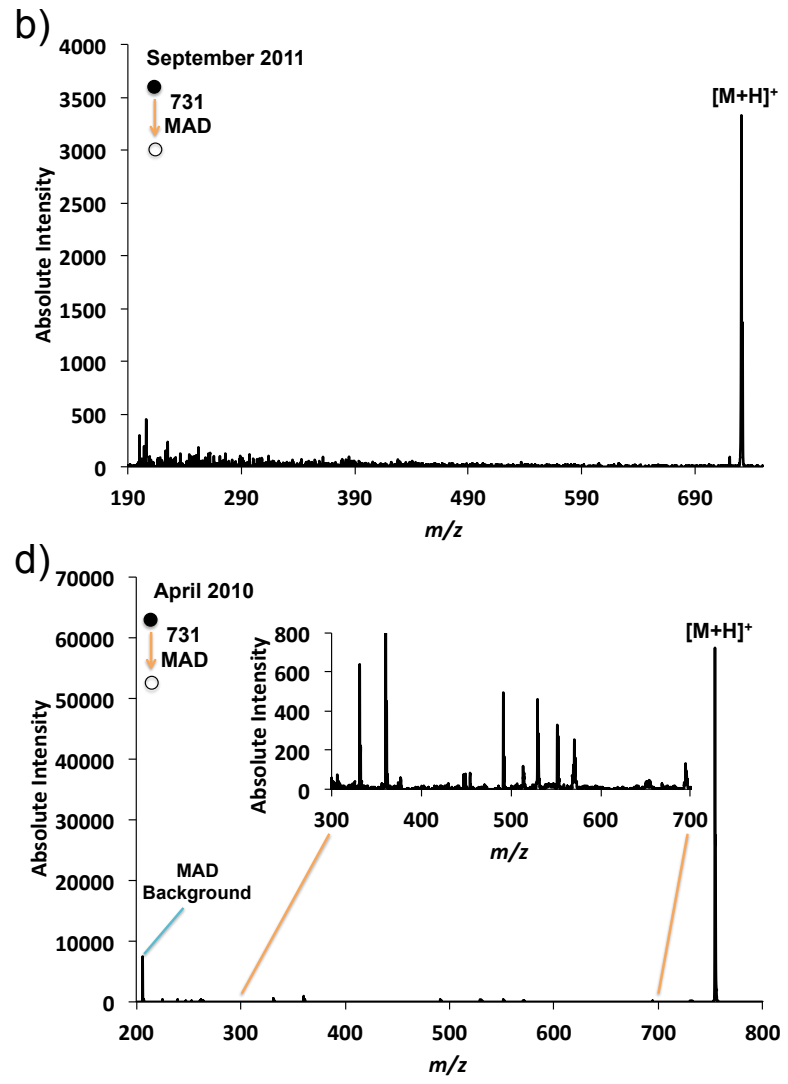

Figure 2.1. Comparison of the isolation and He MAD of $[\mathrm{M}+\mathrm{H}]^{+}$sphingomyelin from a,b) an analysis in September 2011 and c,d) from an analysis in April 2010.

To assess whether the instrument was capable of effectively performing lipid analysis for this project sphingomyelin, a test lipid, was analyzed. Sphingomyelin was selected because it had already been fragmented and characterized by Dr. Shannon Cook, 
a previous group member. The results of this initial test and its comparison to the previous group member's results are shown in Figure 2.1. When these two sets of spectra are compared to one another we notice major differences in the MAD fragmentation pattern with the 2011 experiment showing no fragmentation compared to the group of fragments observed in the 2010 experiment. Both the 2010 and 2011 experiments were conducted with identical metastable activation conditions such as exposure time, FAB anode voltage ( $\mathrm{He}^{\mathrm{m}}$ kinetic energy), and $\mathrm{FAB}$ pressure. However, ion conditions were different in an attempt to obtain similar precursor ion abundances. Even after signal optimization, the precursor ion signal in the 2011 experiment could not be improved beyond $1.8 \times 10^{-5} \mathrm{au}$, which explains why the signal to noise ratio of the product ions is below the limits of detection. However, the decrease in product ion signal seemed to indicate that something else was wrong.

Once we observed that the instrument was not producing any significant fragments for sphingomyelin, it was necessary to verify its performance overall and determine if the instrument was capable of producing normal fragmentation for a bettercharacterized compound like bradykinin. Bradykinin was selected because it had been analyzed several times in our lab and is known for producing a consistent fragmentation pattern with MAD that could be used as a reference for system performance. Bradykinin was then analyzed using MAD and compared to data that was taken previously in June 2011. (Figure 2.2) Comparison of these two spectra for bradykinin shows that the spectrum produced in September of 2011 has fewer types of fragments present at lower abundances than what is observed in June. This lack of fragmentation was a clear 
indication that something had changed in the instrument and was causing a large decrease in MAD efficiency.

To correct this decrease in system performance, it was necessary to pinpoint

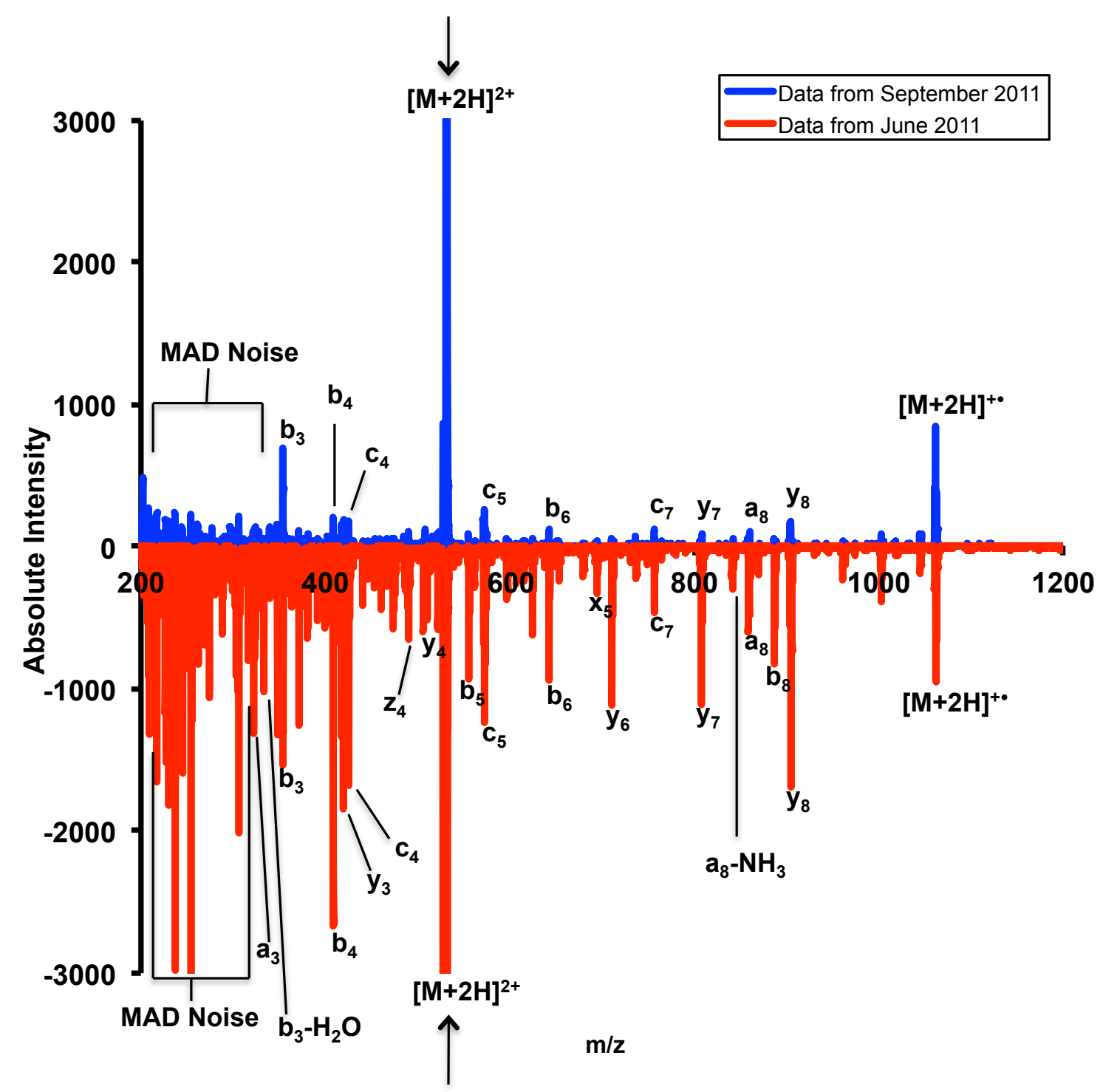

Figure 2.2. Head-to-tail comparison of the He MAD analysis of $[\mathrm{M}+2 \mathrm{H}]^{2+}$ bradykinin taken in June 2011 (in red) compared with the same analysis performed in September 2011 (in blue). 
different areas of the system that were susceptible to change over time. Several possibilities were identified. The first possibility was that the instrument was dirty. Over the course of many experiments deposits of sample analytes can build up on the inside of the instrument in different places including the capillary, the skimmer cones or the inside of the ion trap. These deposits can affect system performance by attenuating electric fields, charging up or by causing electrical discharges. The second possibility is that the FAB gun was not aligned properly in September. The FAB gun that generates the metastable atoms is mounted to lid so that the opening in the FAB gun is directly above the hole in the ring electrode of the ion trap. If the two are not properly aligned then the metastable atoms will either miss the hole or be severely attenuated. A third possibility is that the FAB gun is not producing as many metastable atoms in September as it was in June. Examples of problems that could occur involving the FAB gun are gas leaks, nonoptimized MAD parameters, an overheated resistor, or particulate buildup on the beam aperture or anode of the instrument.

\subsubsection{Cleaning the instrument}

The most commonly recommended procedure for dealing with a sudden decrease in system performance is normally just to clean the instrument. In the case of our mass spectrometer this entailed removing the capillary, skimmer cones, and the ion trap and cleaning them through sonication in a regiment of solvents. The regiment of solvents normally included sonication in 50:50 2-proponal/water, followed by 50:25:25 2proponal/acetonitrile/cyclohexane, and finally sonication in a solution of methanol. If this was not sufficient to remove the particulates then the individual pieces could also be scrubbed with a more abrasive substance like aluminum glycol as well. After this process 
was complete, the instrument was reassembled and testing was performed on bradykinin to see if performance had improved. Figure 2.3 shows the comparison of this new data after the cleaning compared with the data taken in June 2011. This spectrum shows that there was no improvement and maybe even a poorer performance after cleaning the instrument. For example, when compared to the data from June 2011 this new data, after cleaning, produces much less fragmentation and lacks all the fragments between $\mathrm{m} / \mathrm{z} 305$ to 500 and $m / z 550$ to 900 . This observation suggests that there wasn't a significant amount of contamination on the electrodes obstructing the instruments ability to work properly and that any decrease in instrument performance after cleaning is probably due to the way the instrument was reassembled in sensitive areas like the snug reattachment of the cables around the trap. This new data did create some fragments and produces $\mathrm{x}_{3}$ and $z_{9}$ fragment that were not present in the June 2011 data as well as a $y_{8}$ fragment that was the only match between the two. The abundance of the $\mathrm{x}_{3}$ fragment was greater (Figure 2.3) and in some cases wider (Figure 2.6) then other peaks in the same spectrum. The shape of the peak in Figure 2.6 was not investigated in depth during the experiment but is significantly different than the other product ions and is consistent with a multiply charged product ion fragmenting during ejection from the ion trap. When labile ions acquire internal energy during resonant ejection, they often fragment during ejection and lead to peak fronting because the fragments are lower in $\mathrm{m} / \mathrm{z}$ and eject more quickly than the precursor ion from which they originate. However, a multiply charged product would fragment into larger $\mathrm{m} / \mathrm{z}$ products, thereby causing the broadening toward the higher masses that we see in Figure 2.6. 
This was a perplexing result, as it appeared that cleaning the instrument caused further variation in the fragmentation pattern. The variation in fragmentation pattern between this new data after the cleaning, the data taken in September 2011 and the data

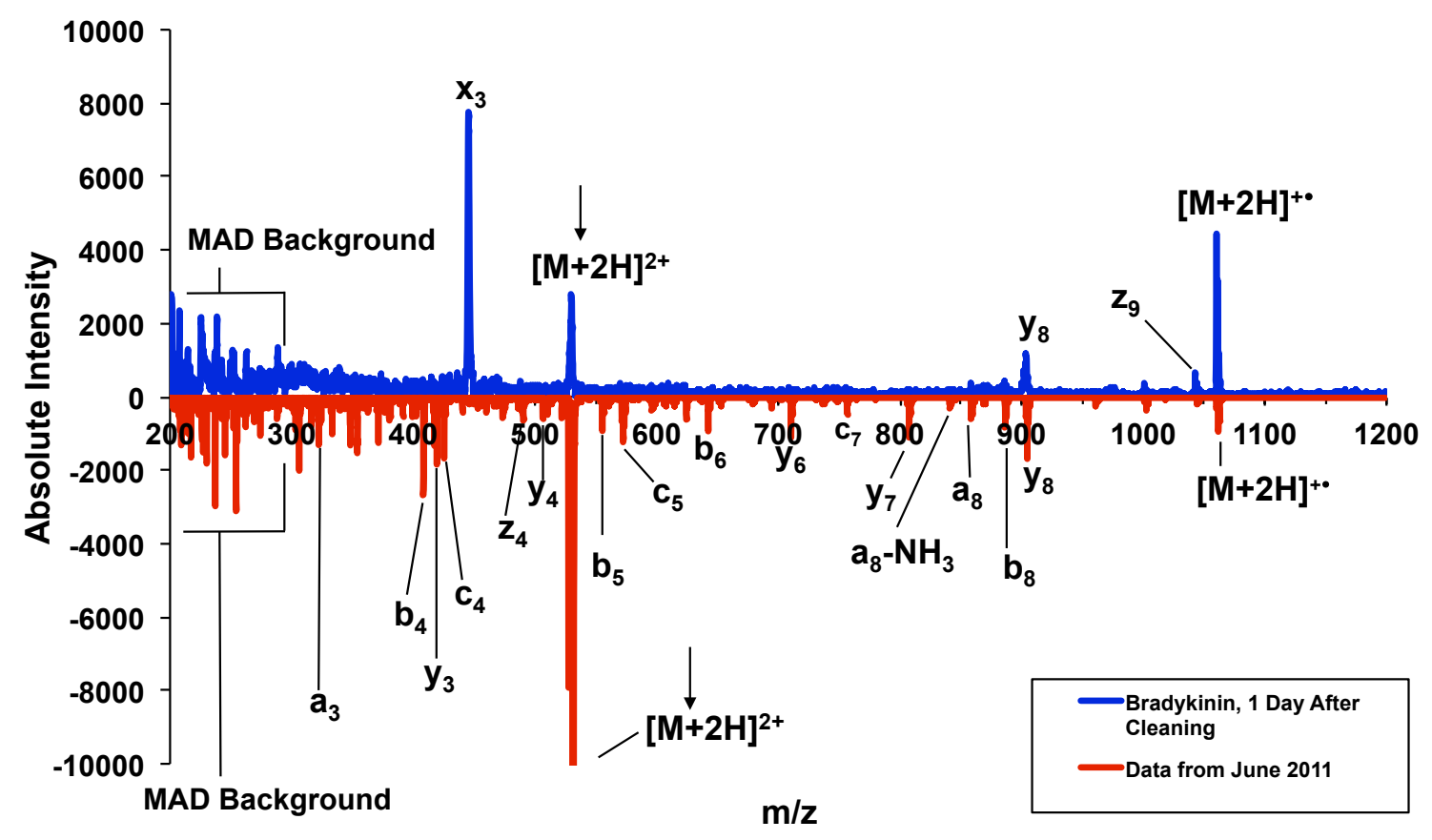

Figure 2.3. Head-to-tail comparison of the He MAD analysis of $[\mathrm{M}+2 \mathrm{H}]^{2+}$ bradykinin taken in June 2011 (in red) compared with the same analysis performed 1 day after cleaning the instrument (in blue).

taken in June 2011 then led us to question whether or not the instrument was producing consistent data.

The instrument was tested again three days after the instrument was cleaned.

Figure 2.4 shows that three days of pumping provided signal differences in performance.

For example we see that the $\mathrm{x}_{3}$ peak present one day after cleaning disappears by the third day and three $\mathrm{c}$ fragments appear that were not present before. We also see that the noise 
level appears to increase as one goes from the first day after cleaning to the third consistent with increased Penning ionization of background pump oil.

The observation that the spectra were not exactly the same between one and three days after cleaning was somewhat cause for concern. Also, if the instrument was cleaned thoroughly then the optimal parameters could also shift based on the fact that there is now less particulate matter coating the ion optics and ion trap. The next objective was to try and optimize the different instrumental parameters to see if these could improve performance.

\subsubsection{Adjustment of instrumental and MAD variables}

There are several different parameters that need to be accounted for in order to achieve a successful MAD result. To optimize the instrument a parameter was selected and altered incrementally over a series of experiments on bradykinin. Parameters tested during optimization included instrumental variables (i.e. ion optics voltages or ion accumulation time) and MAD variables ( i.e. FAB gas pressure, FAB voltage, MAD beam pulse length, MAD beam pulse width). The spectra were then compared to one another and the abundances of similar MAD fragment types were used as the measure of MAD efficiency or instrument performance. The parameter value that produced the largest variety and abundance of fragment types was selected as the optimum value for the parameter. A monovariate calibration method was selected in this case for its ease of use and more intuitive method of execution. The optimal settings as determined using the procedure above are shown in Figure 2.5 along with the spectrum that those parameters were able to produce. After optimization there was some change in the parameters 
compared to those that were utilized in the earlier test. For example, a better result was

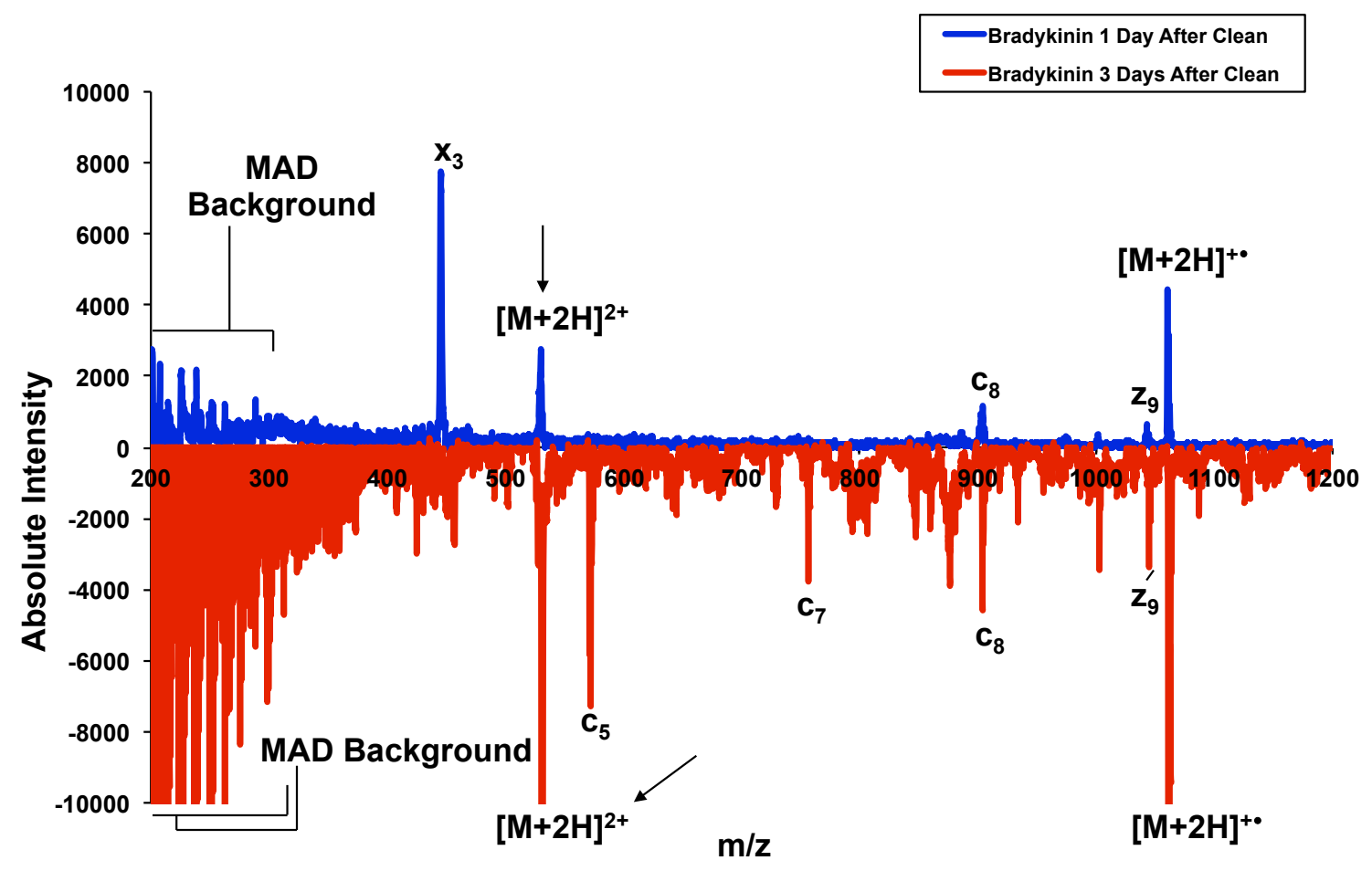

Figure 2.4. Comparison of the He MAD analysis of $[\mathrm{M}+2 \mathrm{H}]^{2+}$ bradykinin one day (in blue) 3 days after cleaning the instrument (in red).

achieved using a lower MAD pulse time and MAD pulse width which shifted from 100 Pulse Time (PT) and 90 PW Pulse Width (PW) in the first experiment to 60 PT and 50 PW in the optimization experiment. In this case the PT is the amount of time allowed for fragmentation in the mass spectrometers cycle whereas the PW is the amount of time that the FAB gun is on during the overall PT. In this case, the spectrum produced by the optimized data appears to be closer to the reference data then it was after cleaning alone. The peaks between $m / z 700$ and $m / z$ 890, which were missing after cleaning the instrument, could now be recovered. Important fragments shared between the reference 
and the optimized spectra in this range include $\mathrm{y}_{8}, \mathrm{~b}_{8}, \mathrm{a}_{8}, \mathrm{a}_{8}-\mathrm{NH}_{3}, \mathrm{y}_{7}, \mathrm{c}_{7}$ and $\mathrm{y}_{6}$ fragment. Despite these similarities there was still some fragments missing in the $\mathrm{m} / \mathrm{z} 400$ to $\mathrm{m} / \mathrm{z}$ 650 range for the optimized data. However, at this point the instrument was still producing a spectrum that was much closer to the reference than it was before. Over the course of this optimization experiment another set of reproducibility spectra were also recorded and analyzed. This experiment compared the fragmentation produced with the optimized parameters one and three days after these parameters were determined. (Figure 2.6) From this experiment we can see that the spectra produced after three days are more consistent with best conditions in June 2010. MAD background is expected to vary over time because it will increase with $\mathrm{He}^{*}$ production and decrease after prolonged sputtering under vacuum. The amount of MAD noise that we observe changes over time. there will be more residual pump oil in the trap and the noise will be the greatest. As time goes by, the pump oil will be reacted or sputtered away as it continues to interact with the metastable atoms causing the noise to decrease to some steady state value. Because of this observation, the FAB gun is normally allowed to 'burn' or operate continuously for a set period of time before an experiment in order to reach the lower noise plateau before an experiment begins.

Once the instrument had been optimized on bradykinin, I again ran sphingomyelin. This new experiment still showed no evidence for MAD of the sphingomylein precursor. To be sure that sphingomyelin wasn't incompatible with MAD, another lipid, N-Heptadecanoyl-D-erythro-sphingosine, was analyzed too. This lipid also failed to produce any fragments. The MAD spectra for sphingomyelin and NHeptadecanoyl-D-erythro-sphingosine are shown in Figure 2.7. Now that it had been 
June 2011 Parameters

Instrument Parameters

Skim 1

Capillary Exit Offset

Octopole

Octopole $\Delta$

Trap Drive

Skim 2

Capillary Exit

Octopole RF

Lens 1

Lens 2

MAD Parameters

FAB Gas Pressure

FAB Voltage

MAD Pulse Width

MAD Pulse Time
September 2011 Parameters

Instrument Parameters

Skim $130 \mathrm{~V}$

Capillary Exit Offset $\quad 50 \mathrm{~V}$

Octopole $2.84 \mathrm{~V}$

Octopole $\Delta \quad 1.76 \mathrm{~V}$

Trap Drive $\quad 40 \mathrm{~V}$

Skim $2 \quad 5.7 \mathrm{~V}$

Capillary Exit $\quad 100 \mathrm{~V}$

Octopole RF 267.2 Vpp

Lens 1

$-2.4 \mathrm{~V}$

$-35.7 \mathrm{~V}$

MAD Parameters

FAB Gas Pressure

FAB Voltage

$3.1 \times 10^{-5} \mathrm{mbar}$

$50 \mathrm{~ms}$

$90 \mathrm{~ms}$

$100 \mathrm{~ms}$

MAD Pulse Width

$60 \mathrm{~ms}$

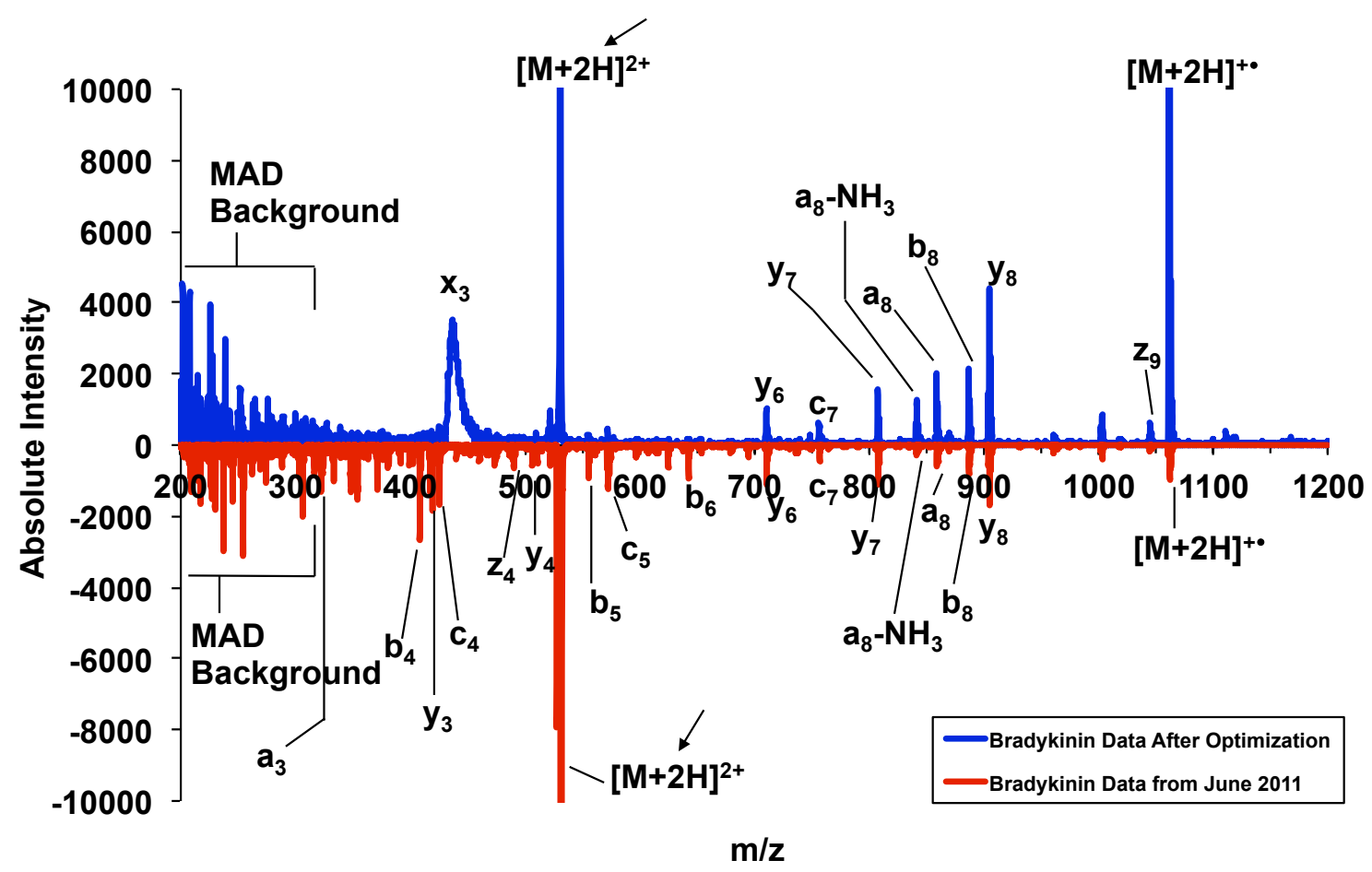

Figure 2.5. Comparison of instrumental parameters before and after optimization (top) as well as the He MAD spectrum of $[\mathrm{M}+2 \mathrm{H}]^{2+}$ bradykinin produced by these new parameters (bottom). 


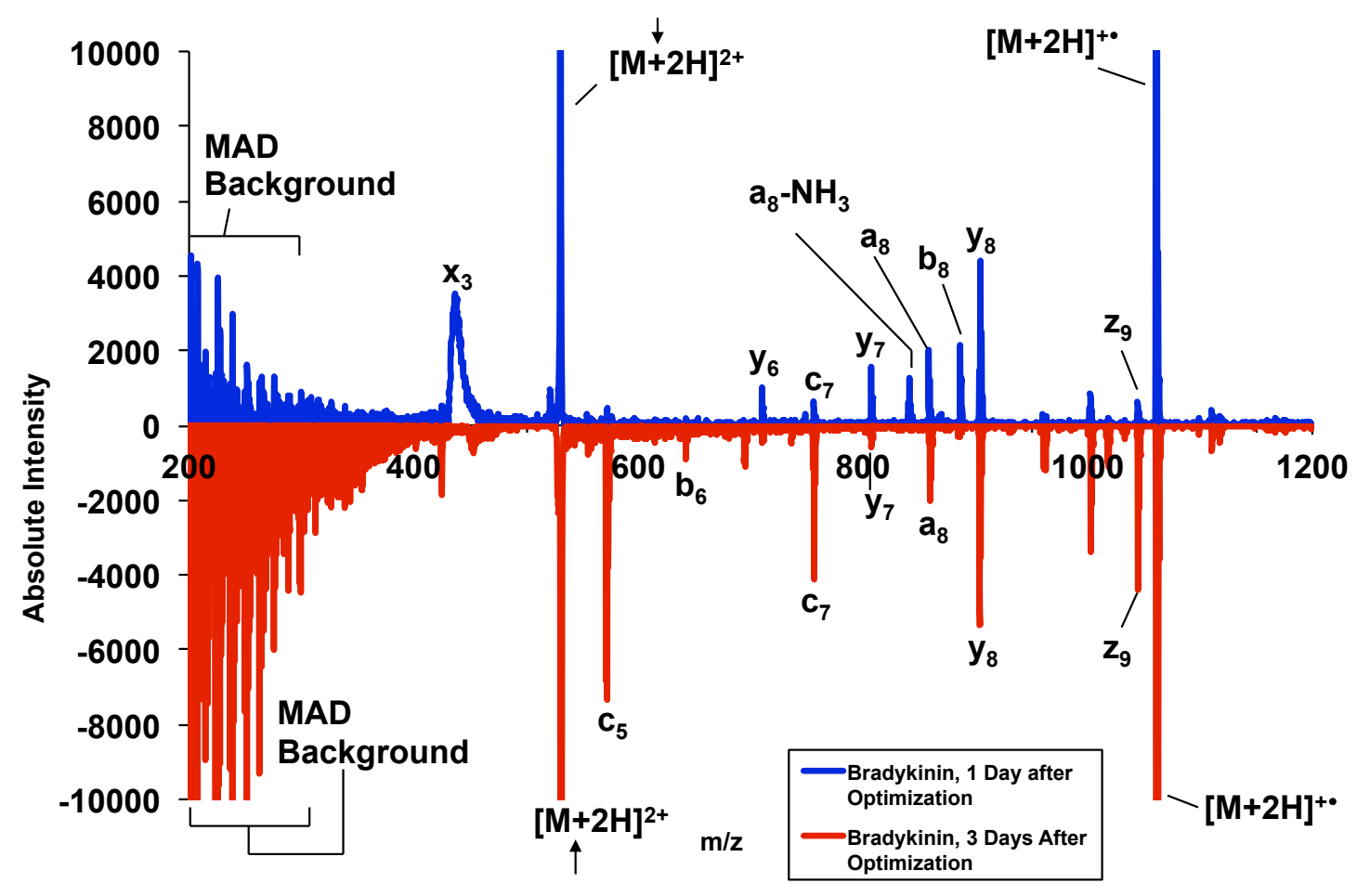

Figure 2.6. Comparison of the He MAD spectrum of $[\mathrm{M}+2 \mathrm{H}]^{2+}$ Bradykinin one day (in blue) and three days (in red) after the optimization experiment.

confirmed that cleaning and optimization of instrument was still not sufficient to cause fragmentation of the lipid, it was necessary to consider other factors.

\subsubsection{Adjusting the alignment of the FAB gun}

The alignment of the FAB gun with respect to the opening in the ring electrode of the ion trap was also considered. The position of the gun relative to this opening is important because if the gun is not aligned properly then the metastable atom beam will be attenuated. The alignment of the FAB gun is normally accomplished using a homemade alignment rod. (Figure 2.8) This alignment rod was machined so that the narrow end of the rod fits into an aperture in the bottom of the FAB gun and wider end 
fits into the hole in the ring electrode. Therefore, if the larger end of the rod is placed into the hole in the ring electrode, the FAB gun can be positioned on the lid to center it above
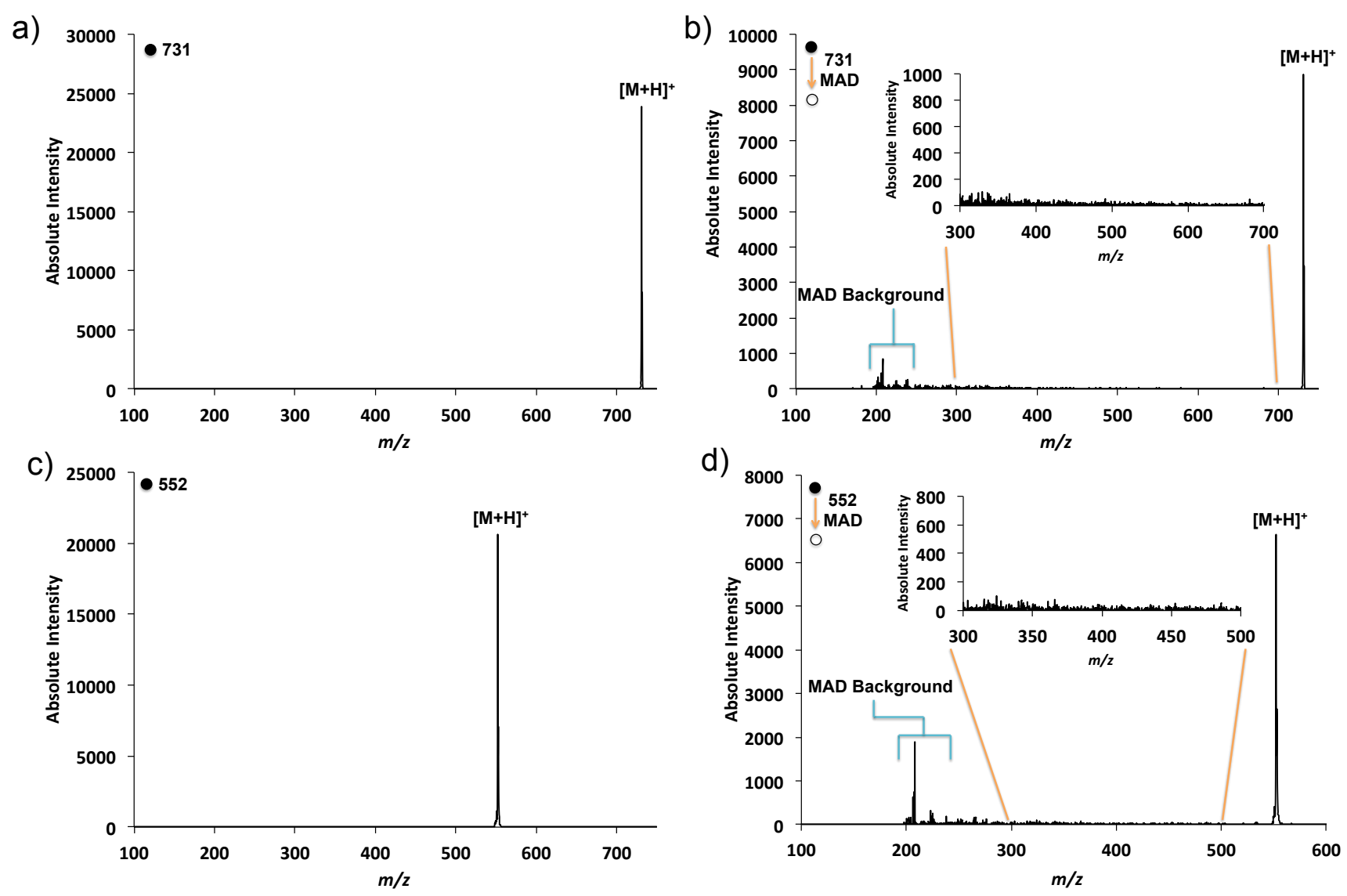

Figure 2.7. Comparison of the isolation and $\mathrm{He} \mathrm{MAD}$ of $[\mathrm{M}+\mathrm{H}]^{+}$of $\left.\mathrm{a}, \mathrm{b}\right)$ sphingomyelin and c,d) N-heptadecanoyl-D-erythro-sphingsine. All data taken three days after cleaning the instrument.

the ion trap. It is important to note that the hole in the ring electrode does not significantly influence the ion traps ability to retain or control ions. The problem with this alignment technique is that the hole in the ring electrode is not circular, it is actually more oblong or oval and the position of the rod described above can flex side to side by several degrees. 
The second indication that the FAB gun may have been misaligned was the observation that the burn marks on the ring electrode were uneven. (Figure 2.9) Burn marks are a long-standing observation in mass spectrometry and ion optics and is typically associated with low-energy ion deposition $(<1 \mathrm{keV})$. High-energy ion deposition usually causes sputtering of metal parts. The center of the burn pattern then is expected to be shiny with darker deposits surrounding it. In this way one can get some

a)

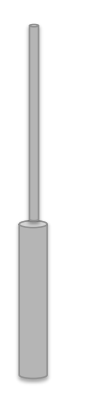

b)

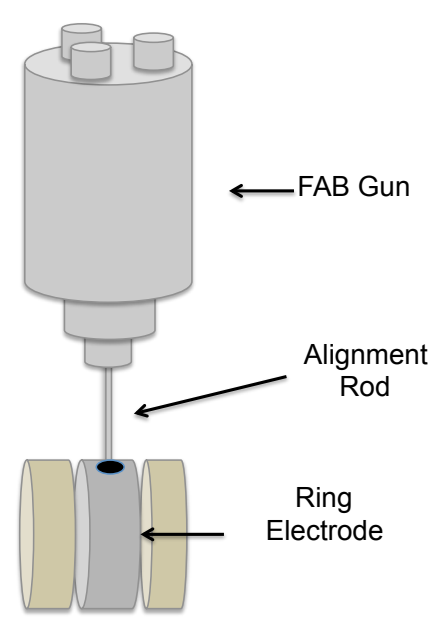

Figure 2.8.Visualization of a) the alignment rod used to center the FAB gun during installation after removing the mass spectrometer's lid and b) the rod in use during an alignment procedure.

idea of where the metastable beam is going based on the location of the burn marks. After the experiments in September 2011, I noticed an asymmetrical burn pattern on the ring electrode. Because the FAB gun has a radially symmetrical orifice (1 mm hole) or aperture one would expect a spherical burn pattern for emanating neutrals. The deflection electrodes are expected to distort the burn pattern caused by electrons or ions depending on the orientation of the electrodes. However, a burn pattern that favors one side of the ring electrode over another can be indicative of FAB gun misalignment. To test whether 
lid position was a factor affecting our fragmentation results three different lid positions were selected for testing (Figure 2.10). Because the FAB gun is mounted in the lid, the

a)

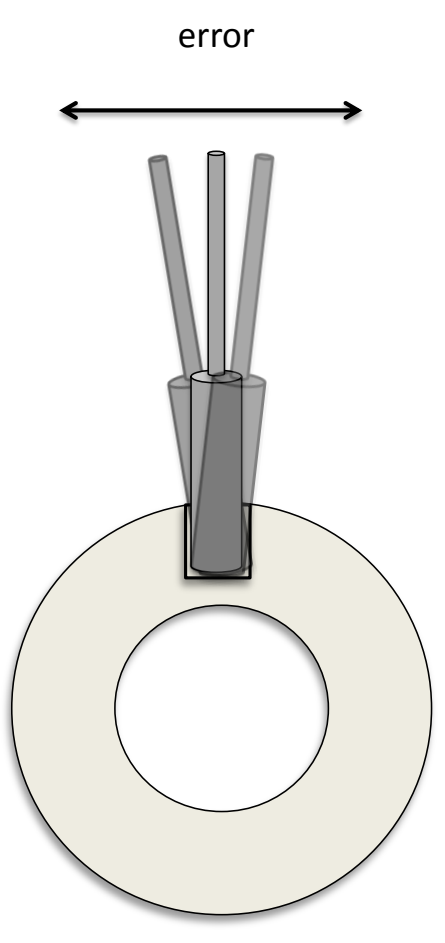

b)

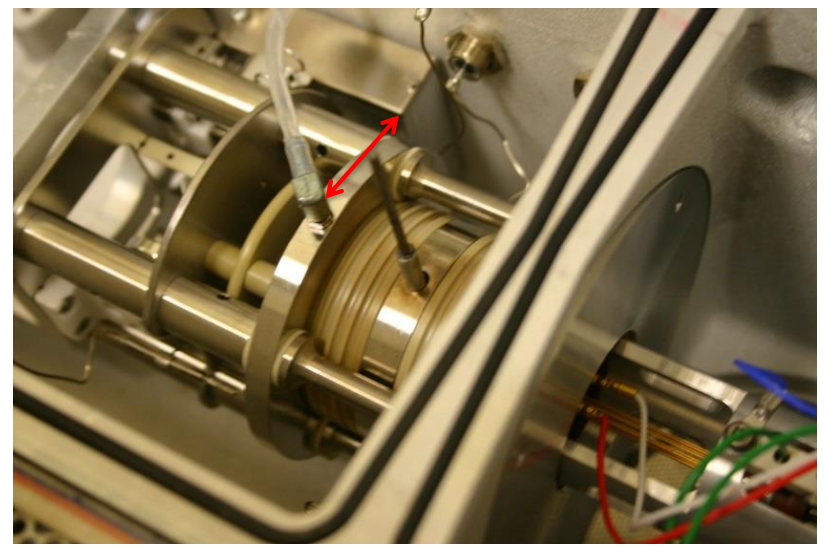

c)

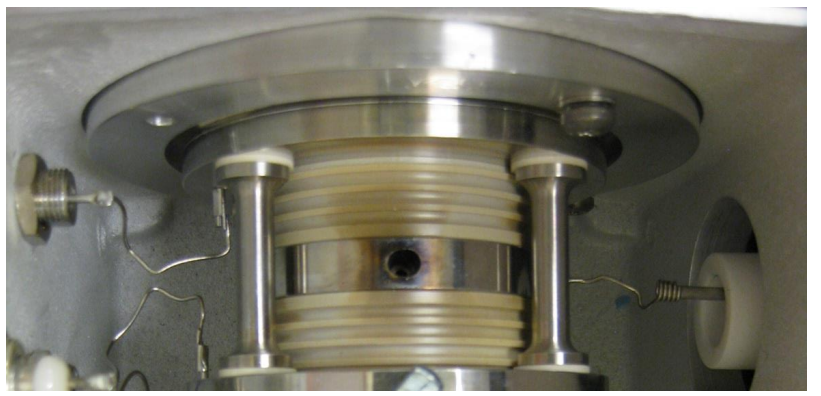

Figure 2.9. Photographs and schematics to show a) the way that the alignment tool can shift in the ring electrode, $b$ ) the alignment tool once placed in the ring electrode, and c) the ion trap showing the burn mark on the ring electrode.

lid position is the most effective way to align the FAB gun. The left, middle and right lid positions are represented visually by the red, blue and green lines respectively in Figure 2.10. For this experiment the relative direction the lid is moved is based on someone standing in front of the sample introduction portion of the mass spectrometer 
and looking forward. The left position selected was the position acquired using the alignment tool. The middle position was a move to the right half the distance of the hole in the ring electrode or about $1 \mathrm{~mm}$ to the right of the first position. The right position had the lid of the mass spectrometer flush with the side of the chamber, which moved the lid position farther right from the middle position. In essence the FAB was being slid in increments to the right with the goal of finding the distance it needed to move to be properly aligned. At

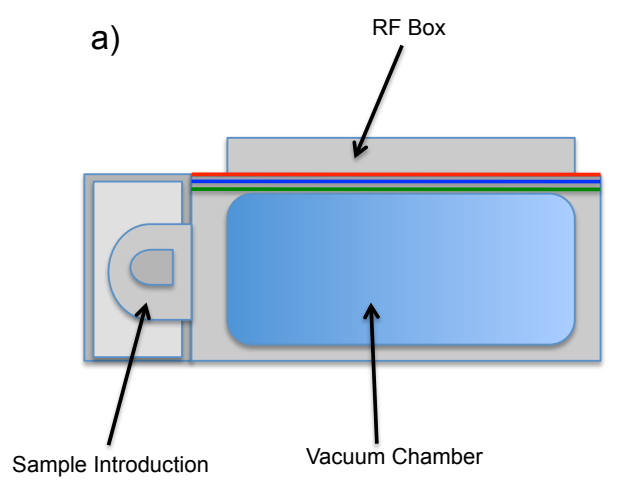

b)
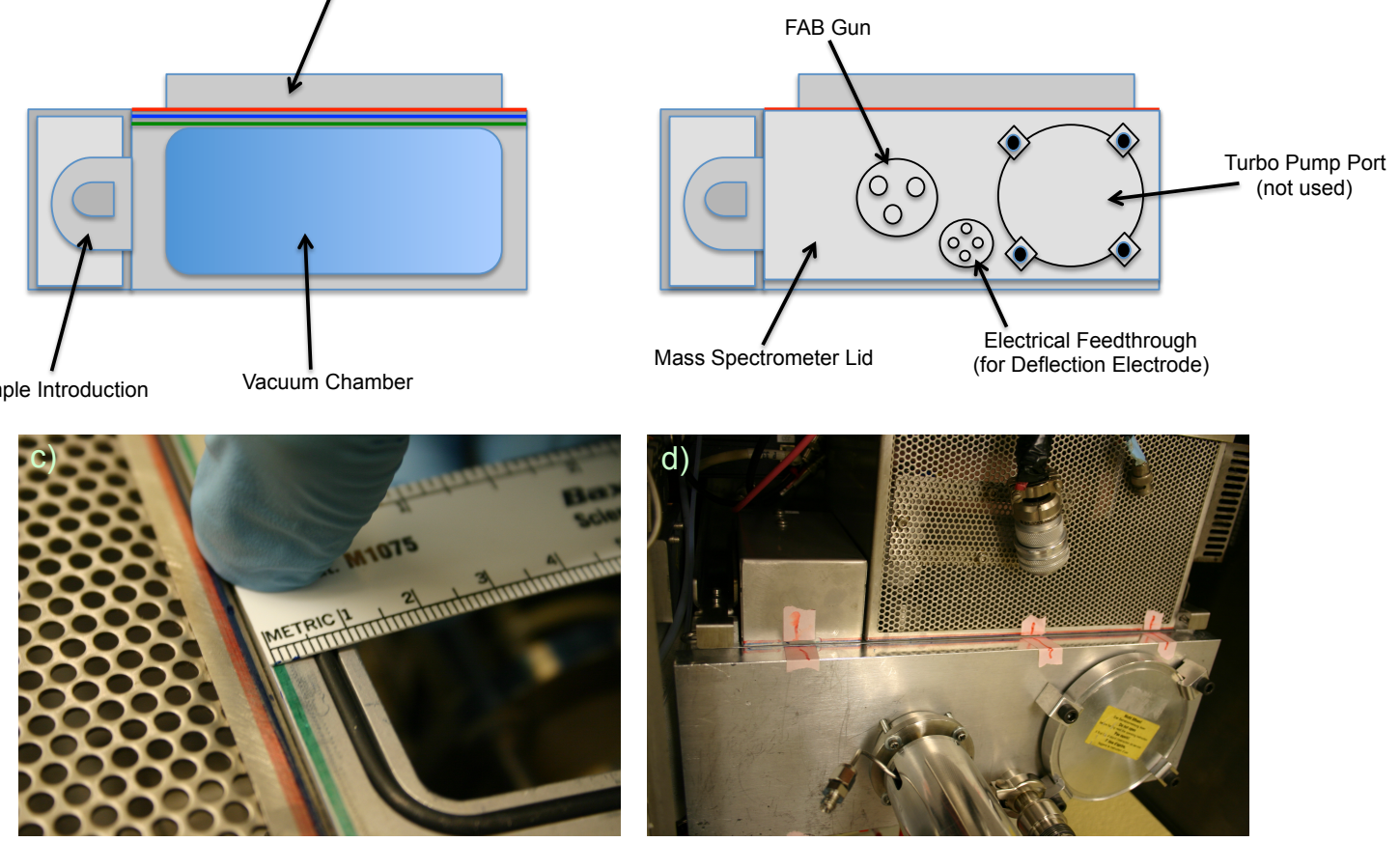

Figure 2.10. Top-down view of the mass spectrometer a) without the mass spectrometer lid and with colored lines representing each of the lid positions. The red line refers to the left position, the blue line refers to the middle position and the green line refers to the right position as described in the text. Also provided is b) an example of how the system would look with the mass spectrometer's lid on in the left lid position, c) a photograph of the three alignment lines as drawn on the mass spectrometer and d) a photograph of the mass spectrometer lid aligned in the left position. 
each position the $[\mathrm{M}+\mathrm{H}]^{+}$form of bradykinin was optimized and analyzed and the variety and abundance fragments with respect to the position of the lid. The spectrum in Figure 2.11a shows the intensity of the $\mathrm{y}_{4}$ fragment at three different FAB voltages and three different lid positions. The right lid position appears to be optimized for this fragment for two of the three energies tested. The effectiveness of this new lid position was then tested by analyzing sphingomyelin, but still showed no fragmentation at all. This meant that although MAD of bradykinin could be improved, MAD efficiency was still inadequate for sphingomyelin thus there must be another reason for the poor instrument performance.

\subsubsection{Analysis of an alternate type of FAB gas}

The next variable that could affect the fragmentation that we observe using MAD is the type of noble gas atoms that is used to generate the metastable atoms. Different noble gas atoms are going to possess different levels of potential energy when their electron is promoted into the excited state. This variation in energy could then allow the selection of the amount of energy you want to put into an analyte for a particular experiment, which could affect the type and degree of fragmentation. In the prior tests the noble gas used for MAD was helium, which has a high-energy metastable state. For the next experiment the type of gas was changed from helium to argon to see if this change in energy delivered to the target structure would cause fragmentation to occur. In this case it may seem counter intuitive to use argon as metastable argon atoms will ultimately deliver less energy then a metastable helium atom. The main reason for utilizing argon in this case was to see if the flux, collision cross section, or increased mass of argon would have 
a beneficial outcome. Whereas collisions between metastable argon atoms and the target may ultimately deliver less energy an increased number of collisions will help to

a)

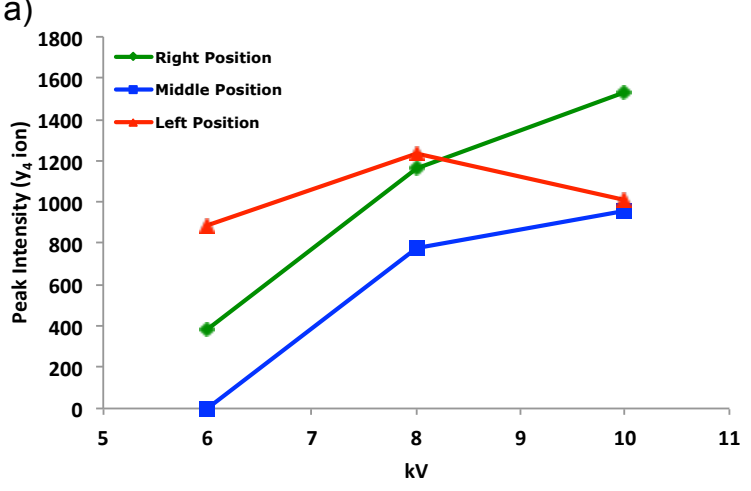

c)

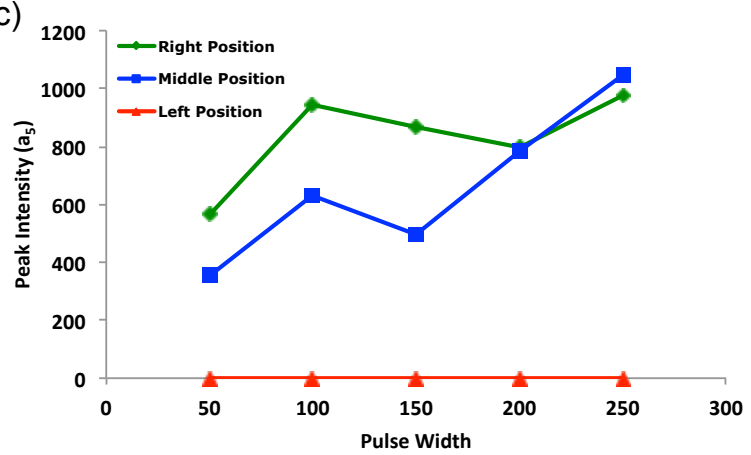

b)

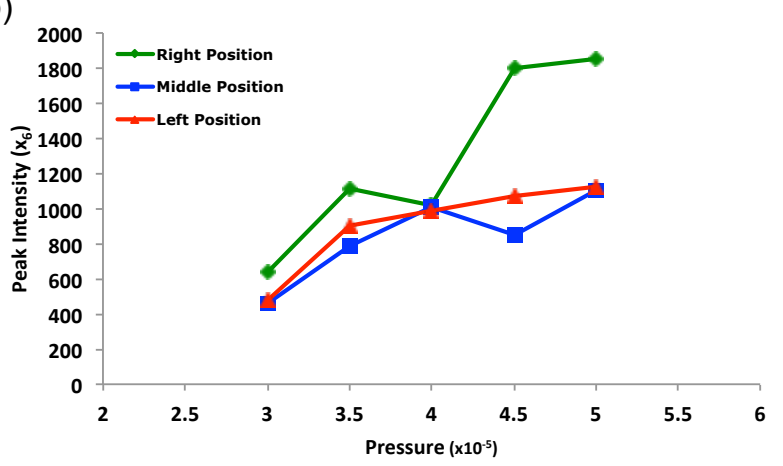

d)

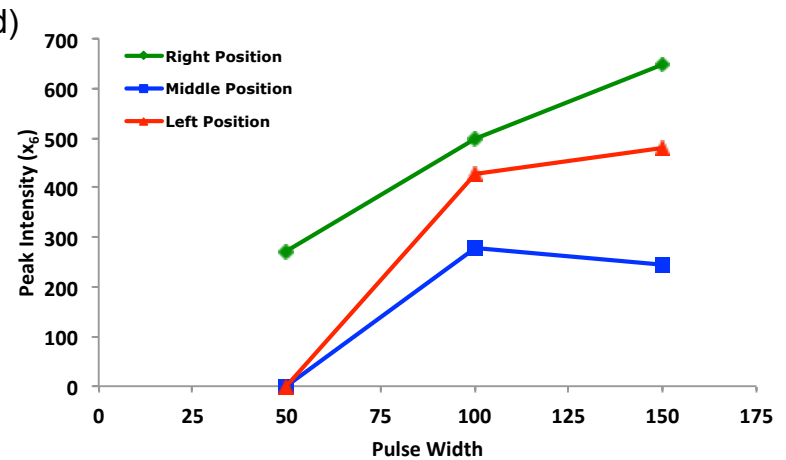

Figure 2.11. Sample of fragmentation results obtained for MAD to optimize the lid position and MAD conditions: a) the change in $y_{4}$ with changing FAB voltage $b$ ) the change in $\mathrm{x}_{6}$ with changing pressure $\mathrm{c}$ ) the change in $\mathrm{a}_{5}$ with respect to pulse width and $\mathrm{d}$ ) the change in $\mathrm{x}_{6}$ with respect to pulse width.

improve sensitivity, as those fragments that are produced will be produced in a higher abundance. Since we are trying to use the argon MAD spectrum as a diagnostic tool to determine if MAD will produce any fragmentation, a gas that can produce more abundant fragments would be helpful. Argon was first optimized on the $[\mathrm{M}+\mathrm{H}]^{+}$precursor of bradykinin. Once the fragmentation of bradykinin was optimized for argon, 
sphingomyelin was again tested. The results of this experiment are shown in Figure 2.12.

Although bradykinin shows fragments, including $-\mathrm{a},-\mathrm{x}$, and -y type ions,

a)

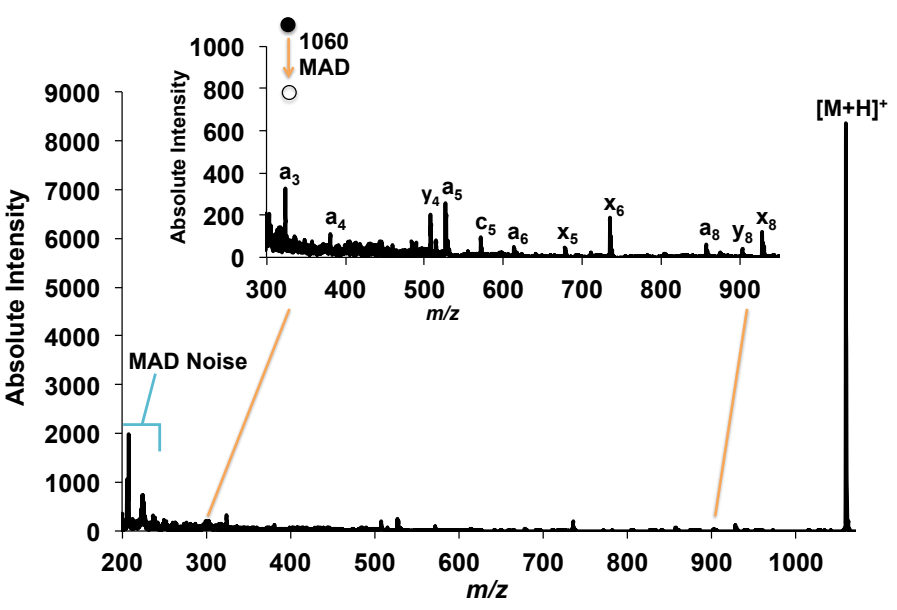

b)

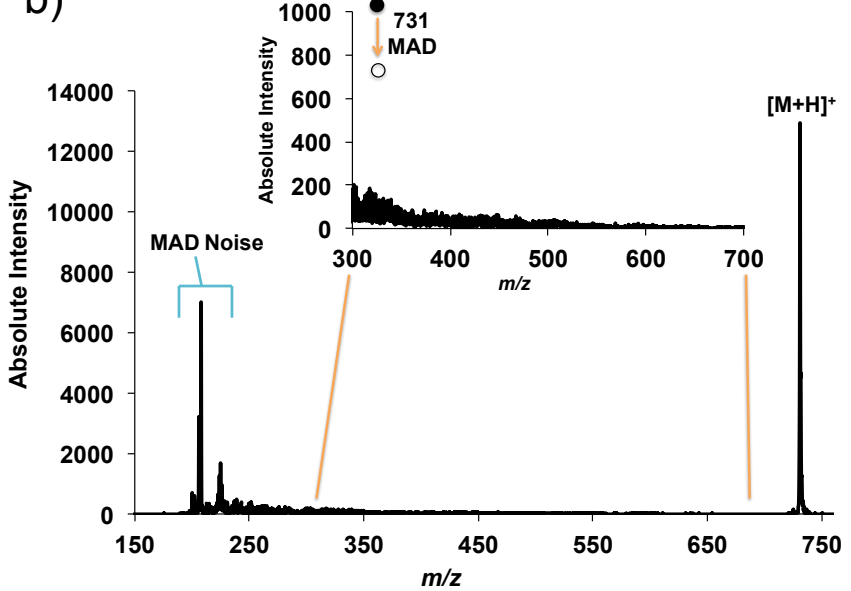

Figure 2.12. The Ar MAD spectrum of a) $[\mathrm{M}+\mathrm{H}]^{+}$bradykinin after optimization and b) $[\mathrm{M}+\mathrm{H}]^{+}$sphingomyelin utilizing the optimized parameters.

sphingomyelin is unaffected. The spectrum for sphingomyelin in this case is flat in the key mass range between $m / z 350-m / z 700$ where the major fragments would be expected.

\subsubsection{Raising precursor ion intensity}

At this point many of the variables that affect the system performance had already been tested. One common occurrence observed in many of the lipid spectra is that their precursor ion abundance tends to be in the range of $10^{4} \mathrm{au}$, which is fairly low. The maximum signal the instrument can obtain before space charge effects occur is in the high $10^{6}$ au range. Thus, we were far below the optimal precursor ion abundance for our experiments. This low precursor ion intensity could be contributing to the lack of 
fragmentation. The obvious solution then would be to increase the precursor intensity and try again. However, this is a much more difficult task then it appears. The precursor intensities shown in the sphingomyelin experiment were achieved after adjusting the isolation mass and width, the instrumental voltages (those listed under instrumental parameters in Figure 2.5), the accumulation time and the chemistry of the sample solution.

The $[\mathrm{M}+\mathrm{H}]^{+}$form of sphingomyelin was fairly difficult to isolate but we decided to continue experimenting on this compound because other lipids that we attempted to isolate like N-heptadecanoyl-D-erythro-sphingosine were just as challenging. Difficulty in isolation is normally attributed to either a labile precursor or a decrease in the accuracy of mass calibration or efficiency of isolation. Sphingomyelin is not a labile compound and has resisted extensive fragmentation even during CID experiments. ${ }^{177,}{ }^{178}$ Thus the mass calibration or isolation efficiency was most likely at fault. If mass calibration is inaccurate then the target mass will not occur at the right $\mathrm{m} / \mathrm{z}$ value and the spectrum will give a false impression that there is less precursor than there actually is. If the isolation efficiency is to low then the isolation window may not be centered and it will eject desired ions during isolation. The remedy for this is to calibrate the instrument with a specified calibration solution. The instrument was calibrated several times over the course of the experiments discussed in this chapter. After calibration we were still unable to isolate the sphingomyelin precursor at intensity above $10^{4}$ au. The lack of precursor intensity after calibration suggested that the isolation efficiency exhibited by the instrument was the best it could do and the lack of precursor intensity could be an instrumental limitation. 
Another approach to increasing isolation abundance is to increase the isolation width. As one increases the isolation width, the isolation window gets wider and precursor ions near the edge of the isolation window, that may have been ejected prior, are allowed to stay in the trap. A larger isolation width then causes an increase in the ion abundance of the precursor. However, increasing the isolation width also increases the range of masses that can fit in the isolation window and thus can allow isotopic peaks to creep in which can compound the spectrum during fragmentation. A trade-off then had to be made between precursor intensity and the isotopic purity of the precursor. The necessity for a large precursor ion abundance caused our experiments to utilize a wider isolation window than would typically be desired and even with a wider window we could only achieve the $10^{4}$ au ion abundance.

Another important variable affecting precursor ion intensity is accumulation time. This parameter controls the amount of time that the trap receives ions from the source before it continues with the rest of the scan functions. The amount of time allowed for accumulation will ultimately determine the maximum amount of ions that are present in the trap. Typically longer accumulation times provide more ion signal. However, a limit is typically reached when space charge effects occur. Space charge occurs when you have too many similarly charged particles in a small area, like an ion trap and columbic repulsion between the ions deviates their trajectory from normal behavior. Space charge can cause a variety of unusual effects. In ion traps, space charge typically manifests as broad and fronting peaks, especially at lower $m / z$ values. This is because ion traps scan ions from the lowest to the highest mass, so there are fewer ions remaining and therefore less space charge at the end of the scan. The goal here as elsewhere, is to identify an 
accumulation time that maximizes the number of ions in the trap without causing a noticeable space charge effect. The best accumulation time was around 20 milliseconds for lipids.

Additional variables increasing precursor ion intensity are the ion optics and trap voltages as discussed in Figure 2.5. These voltages control how efficiently the instrument is able to transfer the ions to the trap (the ion optics) and when they are actually in the trap (the trap voltages). These parameters are adjusted either manually or using the optimization software within the instrument, which performs a series of automated monovariate analyses to maximize signal at a specific target mass. The parameters selected by the instrument were normally used as a starting point for the manual optimization. However, even after spending extended periods of time trying to manually adjust the voltages the precursor intensity could not be increased above the range of $10^{4}$ au.

\subsubsection{Increasing precursor signal through solution chemistry}

Because none of the instrument parameters enabled precursor ion signal greater than the $10^{4}$ au range we considered the chemistry of the sample solution. The solution must have the correct composition of solvents, $\mathrm{pH}$, conductivity and analyte concentration to best electrospray different analytes. Therefore, the electrospray ion source may in fact have been the limiting factor. Sphingomyelin was typically prepared at $60 \mu \mathrm{M}$ in 9:1 methanol/water. In an attempt to increase the $[\mathrm{M}+\mathrm{H}]^{+}$yield of sphingomyelin, I varied the concentration of the analyte and the ratio of the two components in the solvent. First, the ratio of the methanol and water solvent was varied 
from a 1:1 methanol/water solution to a 9:1 methanol/water solution. Next, the concentration of the analyte in the

a)

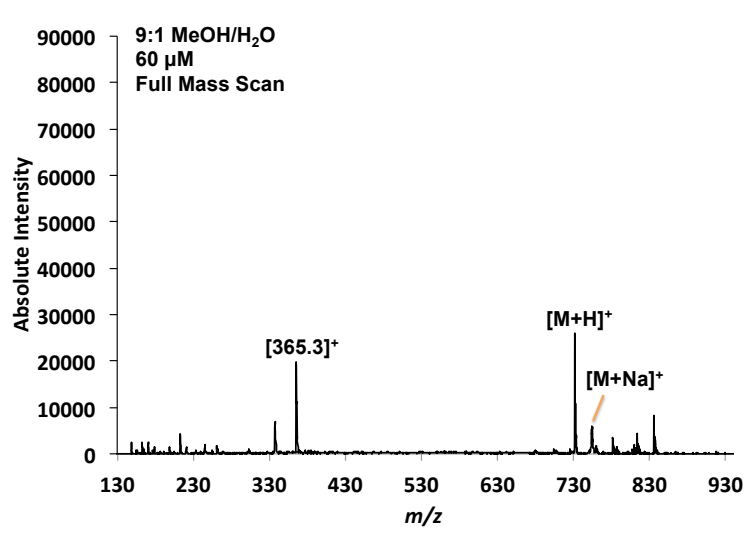

c)

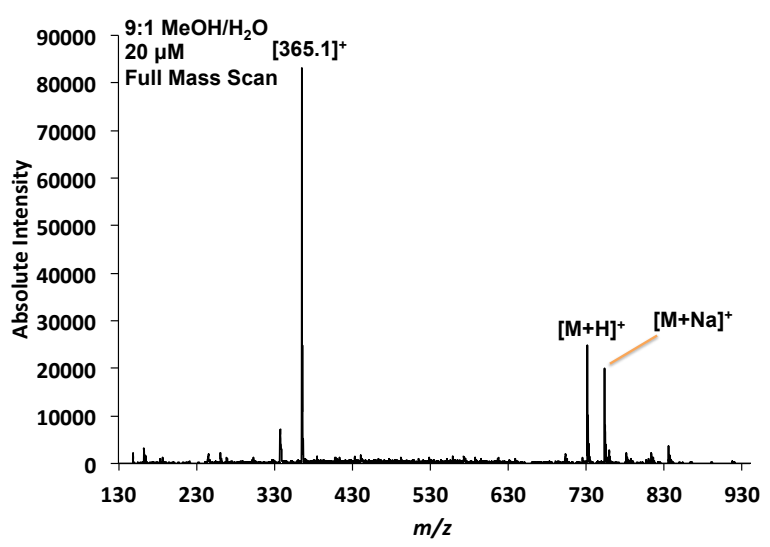

b)

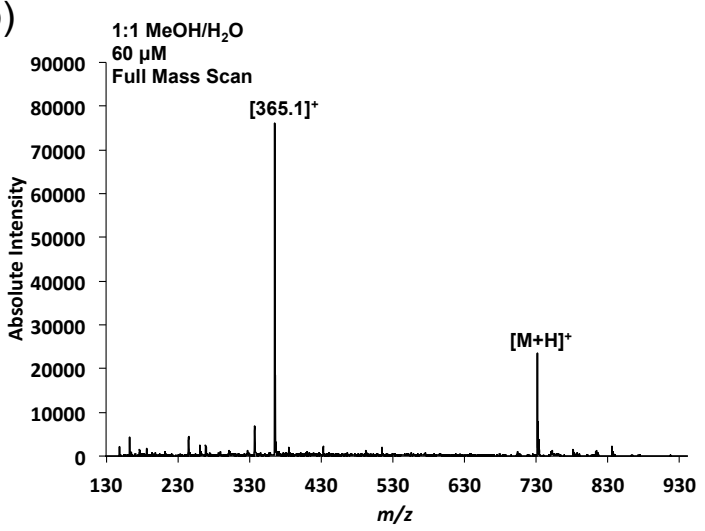

d)

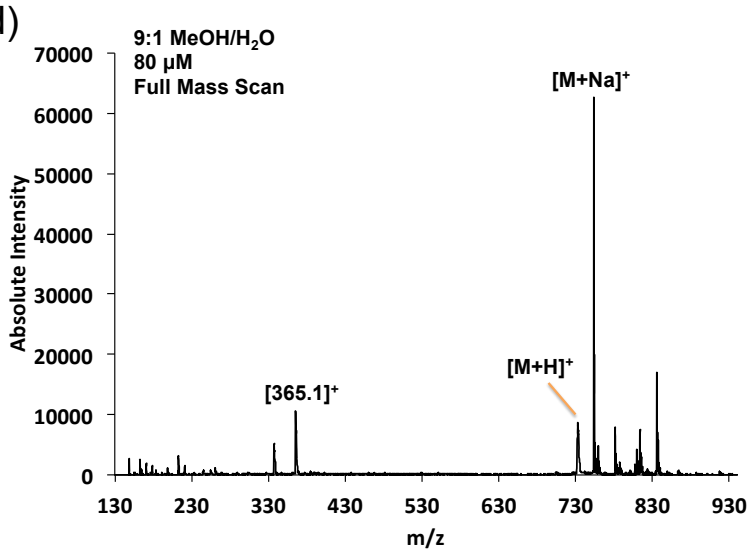

Figure 2.13. An example of a set of full scan ESI spectra produced during the solution chemistry experiments. This set of spectra shows a) the comparison of the full scan ESI spectrum of a $60 \mu \mathrm{M}$ sphingomyelin made in a $9: 1 \mathrm{MeOH} / \mathrm{H}_{2} \mathrm{O}$ solution, b) a $60 \mu \mathrm{M}$ sphingomyelin made in a $1: 1 \mathrm{MeOH} / \mathrm{H}_{2} \mathrm{O}$ solution, c) a $20 \mu \mathrm{M}$ sphingomyelin made in a 9:1 $\mathrm{MeOH} / \mathrm{H}_{2} \mathrm{O}$ solution, and d) a $80 \mu \mathrm{M}$ sphingomyelin made in a 9:1 $\mathrm{MeOH} / \mathrm{H}_{2} \mathrm{O}$ solution.

sample solution was varied from $20 \mu \mathrm{M}$ to $80 \mu \mathrm{M}$. In each experiment the absolute abundance of the singly protonated precursor of sphingomyelin was compared for each combination. The spectra produced for each experiment were not significantly different or abundant over the range studied for the pronated precursor (Figure 2.13). In these 
experimental spectra there were also peaks corresponding to the singly sodiated form of sphingomyelin. Sodiated peaks were not considered in this experiment because the sodium source, the walls of the glass vial, could not be easily regulated and thus not reliably tested. Also, observed was a peak at $\mathrm{m} / \mathrm{z} 365$ which matches the mass of the doubly protonated form of sphingomyelin and varied in intensity based on experimental conditions. The isotopic envelope of this peak however, suggests that it is singly charged leading us to believe that this peak is either a labile fragment of sphingomyelin or a background contaminant. Since the intensity of the singly protonated precursor of sphingomyelin did not vary significantly from experiment to experiment we kept the solution chemistry that we had started the experiment with $(60 \mu \mathrm{M}, 9: 1$ methanol to water), to best solubilize the lipids.

\subsubsection{Exploration of an overheating FAB gun}

Towards the end of these experiments the FAB gun was evidently heating up to the point where one could not comfortably touch it with a bare hand. The FAB gun had never done this before, so we disconnected it from the system and disassembled it to see if we could discern why it was heating up. First, I checked the $1 \mathrm{M} \Omega$ resistor. The FAB gun incorporates a simple resistor to control the current and prevent arcing. If a large amount of current was being pushed through this resistor when the FAB gun was on it could cause resistive heating which could in turn heat the outside shell of the FAB gun. To check this both the resistor and its protective sleeve were examined for damage and signs of overheating. (Figure 2.14) The resistor was not visibly damaged, measured the proper resistance (1 M $\Omega)$ and there were no burn marks on the insulating sleeve. Upon examining the actual source (the bottom portion of the FAB gun) most of the surfaces 
seemed to be relatively clean except for the anode, which showed significant deposition buildup. The build-up was cleaned off both parts through scrubbing with fine

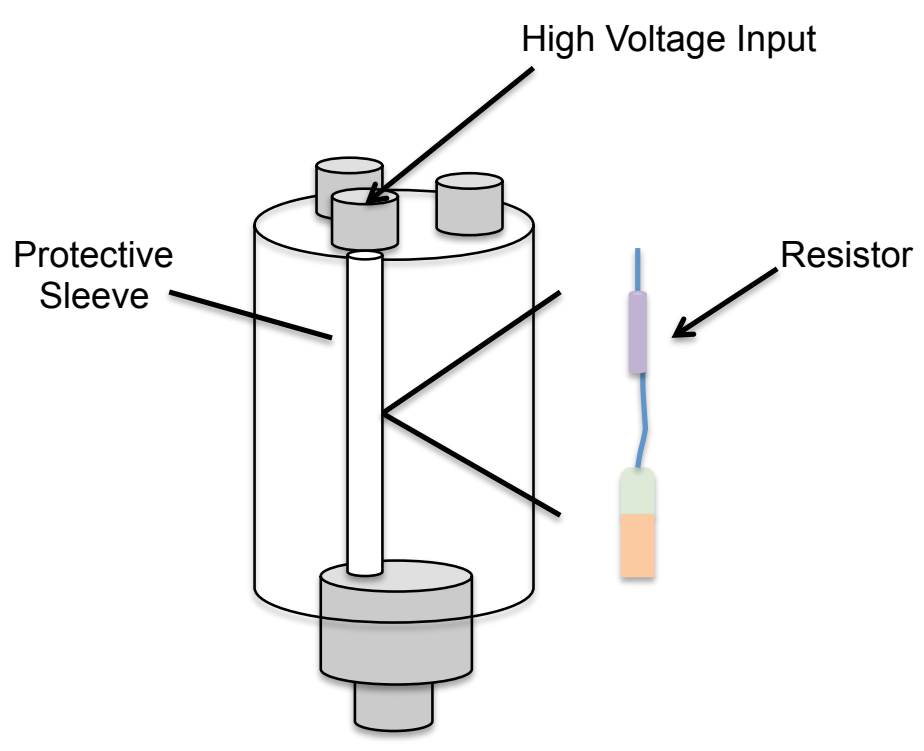

Figure 2.14. Schematic showing the location of the resistor and protective sleeve within the top of the FAB gun.

sandpaper and through sonication in the solvent regiment described earlier. After cleaning (Figure 2.15d) the FAB gun was reassembled and attached to the mass spectrometer. As the gun was reassembled each part was checked to make sure that it fit snuggly in its place and was aligned correctly. If the FAB source is not assembled correctly, in a gas tight manner, then the appropriate pressures can not be attained in the different volumes of the source and the vacuum chamber which would have a negative effect on FAB performance. After re-assembling, realigning and pumping down a MAD experiment was run on bradykinin. However, shortly into the analysis the heating effect was still noticeable and a short break was required to let the FAB gun cool down once again. 

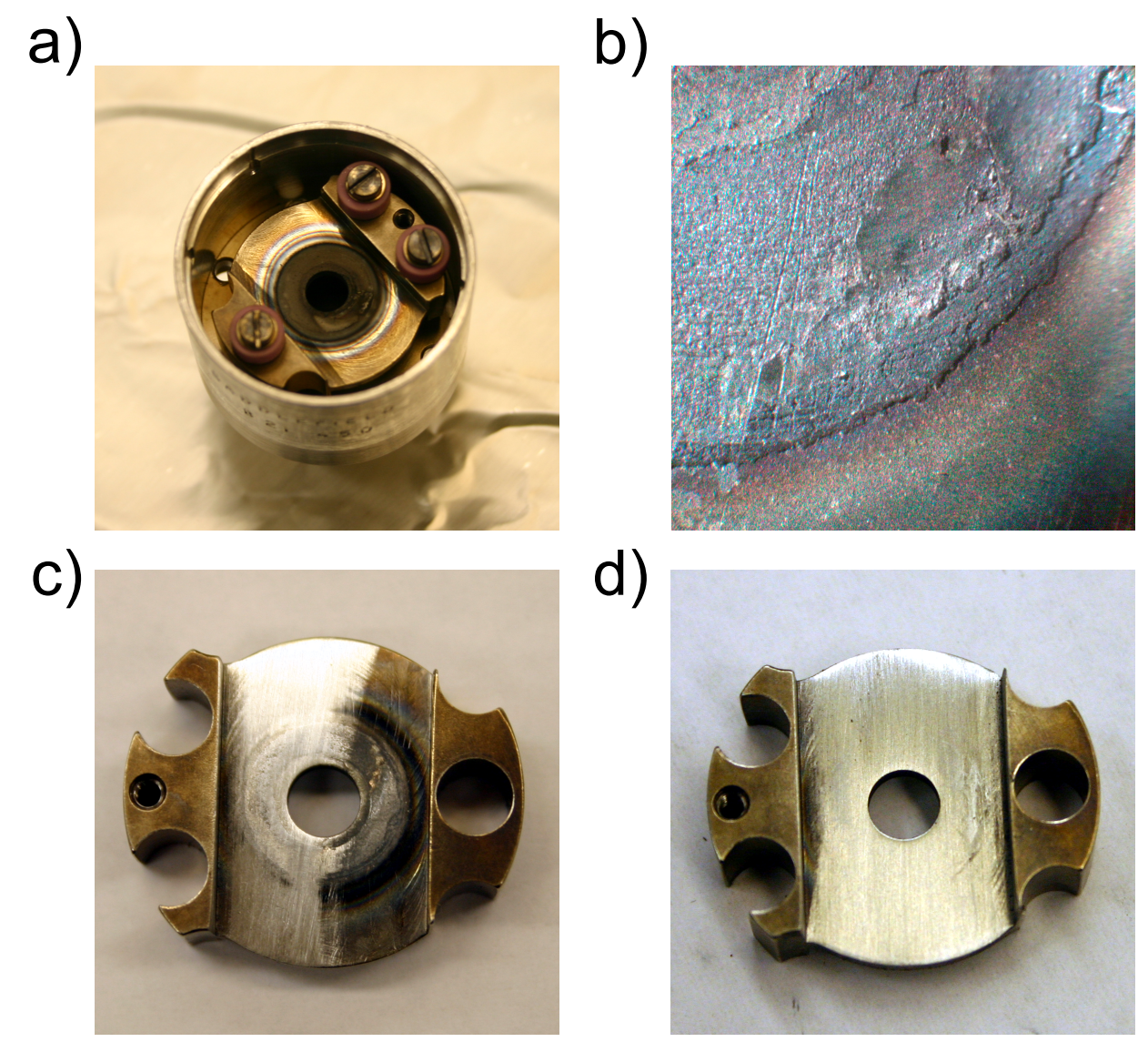

Figure 2.15. Several pictures taken while cleaning the anode of the FAB gun. a) The dirty anode in its place in the nose of the FAB gun before being removed. b) A microscopic image of the contamination coating the top of the anode. c) The anode after the left half has been cleaned. d) The final state of the anode after it has been completely cleaned.

Because the FAB gun did not appear to have anything wrong with it in terms of the cleanliness or the assembly, we were at a loss for a mechanical reason why the FAB gun was generating so much heat during each experiment. One possible non-mechanical explanation was that the FAB gun was not getting enough rest time between pulses during an experiment. (Figure 2.16) Over the course of the many optimization experiments the pulse time and pulse width of the FAB gun had steadily increased from the original $90 \mathrm{~ms}$ pulse width to a $299 \mathrm{~ms}$ pulse width. To decrease the effective duty cycle, the overall pulse time (storage time during FAB pulse) was set to $600 \mathrm{~ms}$, which 


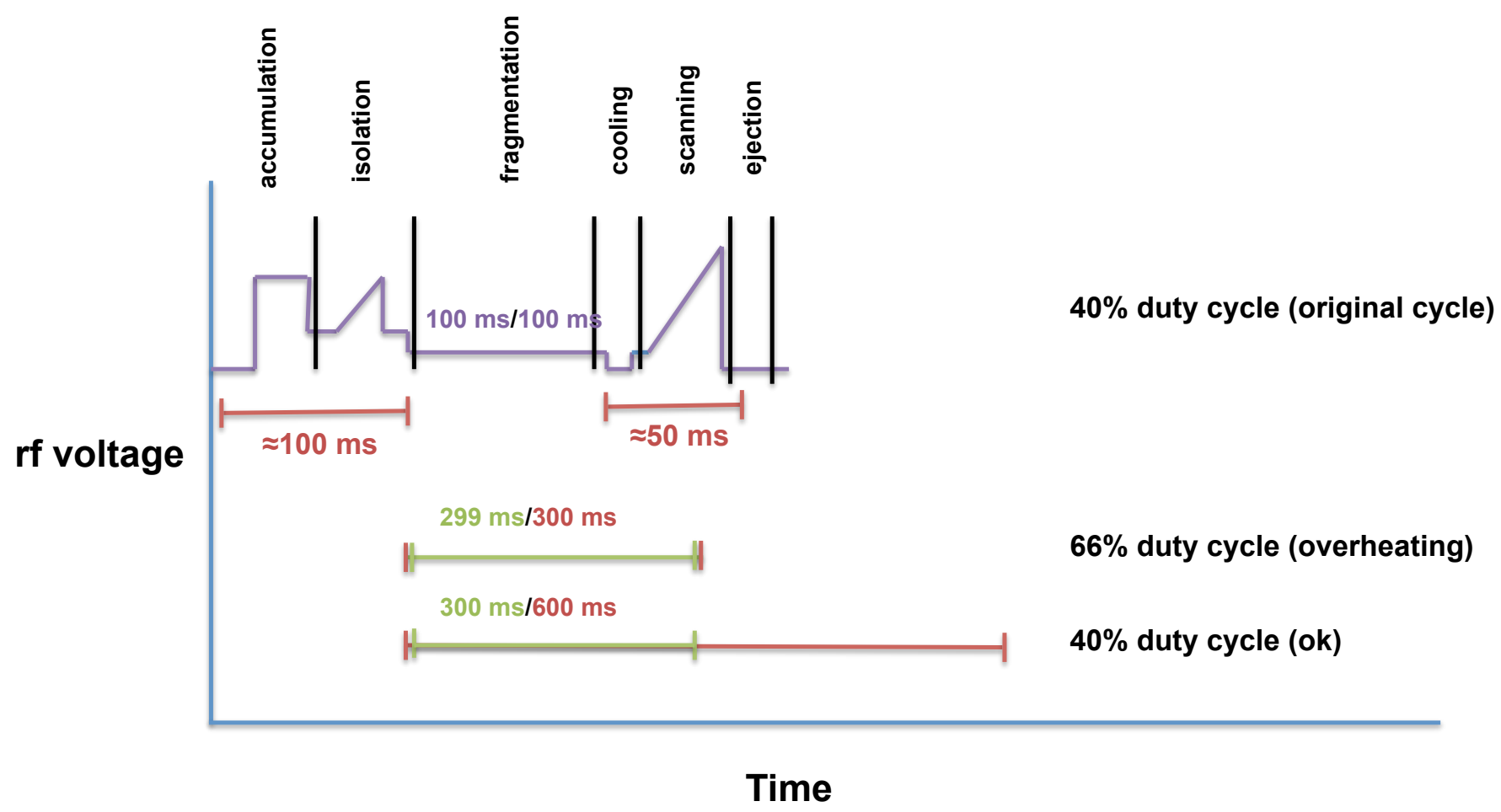

Figure 2.16. Schematic of the duty cycle of the mass spectrometer (in purple), and the experimental pulse times (in red) and widths (in green). The percentage of the duty cycle that the FAB gun is on for each set of experimental parameters is noted on the right.

is twice the length of the FAB pulse time, giving it much more time to cool in between pulses. The FAB gun no longer overheated at the reduced duty cycles.

\subsubsection{Successful fragmentation of target lipids}

Having overcome the heating problem and establishing new pulse width parameters we again tested sphingomyelin. In this case, fragment peaks were produced and despite being a very low abundance, matched the peaks observed in the past. This new data taken in June 2012 was then compared to data taken in April 2010 when the instrument was last working well. (Figure 2.17) Examples of matching peaks from this experiment include $m / z 205.9$, which is a ring structure formed from the cleaved headgroup after a loss of trimethylamine, $\mathrm{m} / \mathrm{z}$ 694.4; the loss of trimethylamine, and 


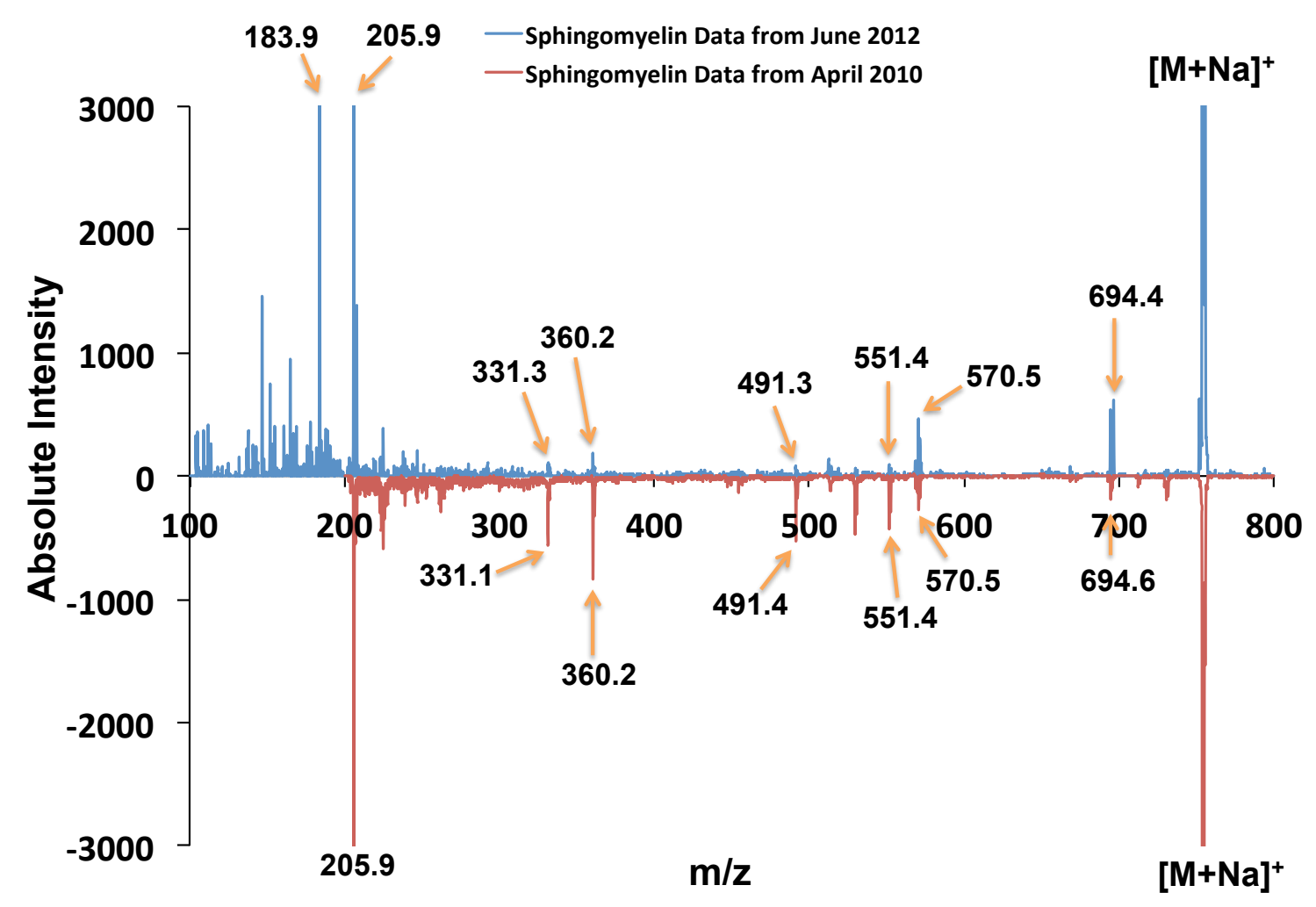

Figure 2.17.Comparison of the He MAD of $[\mathrm{M}+\mathrm{Na}]^{+}$form of sphingomyelin using data taken from June 2011 (in blue) and April 2010 (in red).

$m / z 570.5$; the loss of the entire phosphocholine headgroup. This spectrum was encouraging as it showed that the instrument was still capable of fragmenting lipids, even if the efficiency was less then before.

Because we had exhausted most other options for improving the signal-to-noise, we also optimized the amplifier voltage. Because both signal and noise increase with the applied potential, it is important to maximize the signal-to-noise ratio, not just the signal. Upon optimizing, the multiplier voltage was increased from $1350 \mathrm{~V}$ to $1950 \mathrm{~V}$; a significant increase.

Using all the parameters determined up to this point including the new 
detector voltages we were able to generate usable data from several lipids including sphingomyelin, phosphotidylcholine (POPC), dioleoyl phosphocholine (DOPC) and diarachidoyl phosphocholine (DAPC). An example of the spectra for one of these lipids, POPC, is shown in Figure 2.18. The interpretation of the results of these lipid analysis will be discussed in Chapter 3 .

Our lab group transferred from Ohio University to West Virginia University shortly after these successful sphingomyelin results had been obtained and these new lipids had been analyzed. Our new lab had the opportunity to obtain a more advanced mass spectrometer than the one that I had been using previously. This new mass spectrometer was a Bruker amaZon that had been specifically modified for MAD analysis. For example, the holes in the ring electrode had been pre-drilled in the Bruker factory, and the rf tuning circuit was re-tuned to be optimized with the lower capacitance trap. To test the capabilities of this new instrument several familiar compounds were analyzed again including bradykinin and POPC. The results of the analysis of POPC are
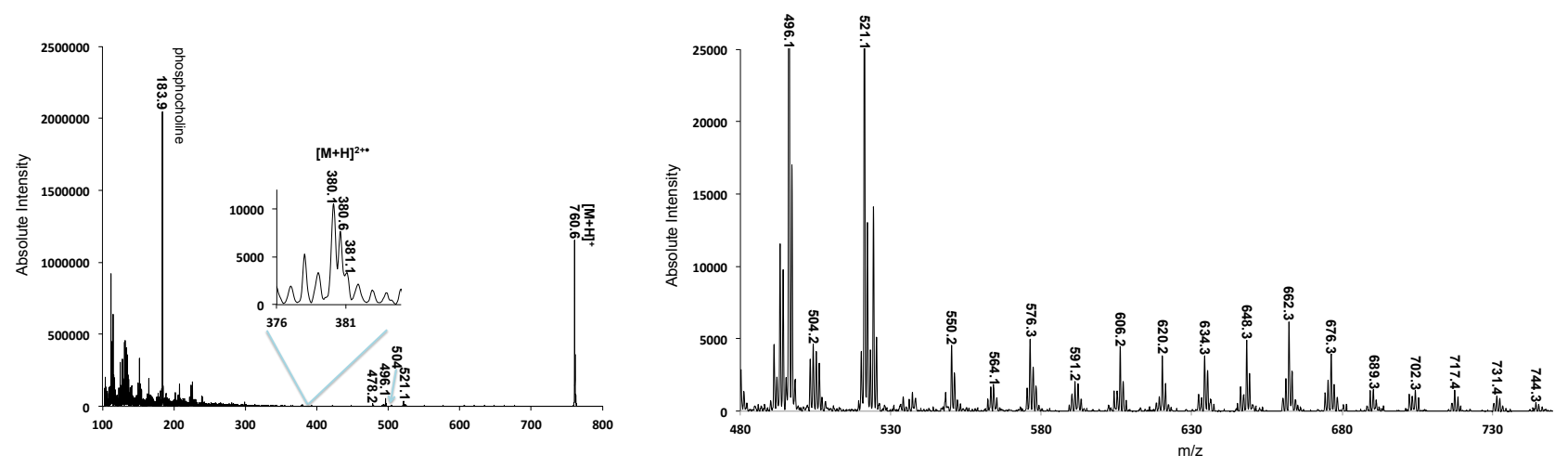

Figure 2.18. The He MAD of $[\mathrm{M}+\mathrm{H}]^{+} \mathrm{POPC}$ taken with the older Bruker Esquire mass spectrometer at the conclusion of all of the maintenance experiments. 
shown in Figure 2.19. The similarity between the old and new ion trap instruments in Figure 2.18 and Figure 2.19, respectively, is a reassuring observation in that the fragmentation is not instrument specific.

\subsection{Conclusion}

This chapter summarizes many months of work devoted to understanding factors that influence the functionality and stability of our modified instrument. Unique challenges of the modified instrument include deposition build up in the FAB gun, overcurrent of the ring electrode and alignment of the FAB relative to the ring electrode. Many different troubleshooting techniques were implemented ranging from mechanical solutions like cleaning the instrument to experimental solutions like altering the different MAD variables. After extensive experimentation and adjustment of the instrument it was able to produce data that had fragmentation patterns similar to those taken of the same compound months earlier. Modification of a new Bruker amazon ion trap, with greater
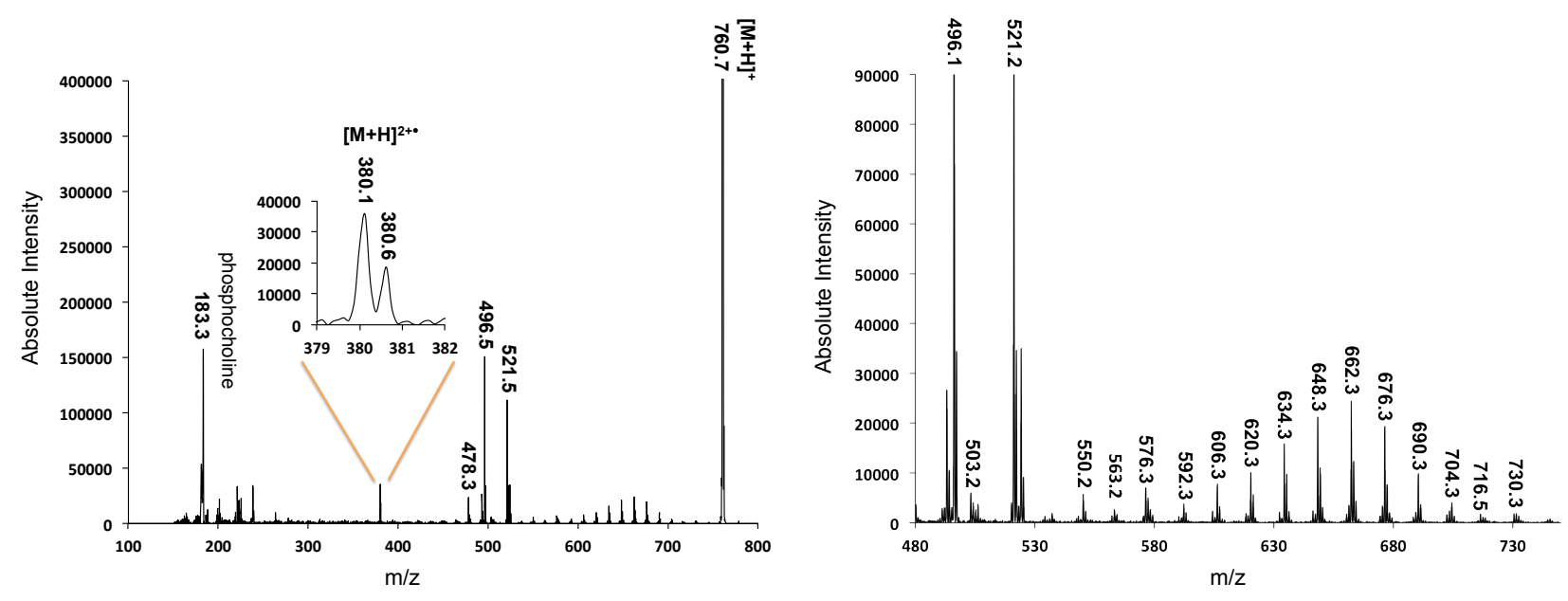

Figure 2.19. The He MAD of $[\mathrm{M}+\mathrm{H}]^{+} \mathrm{POPC}$ taken with the new mass spectrometer shortly after transferring to the new university. 
trapping capacity and better mass resolution provided results that were consistent with the much older Esquire ion trap, but with significantly better signal-to-noise ratio and mass resolution. The agreement between the two instruments gives us more confidence in the peak assignments and conclusions drawn from the older instrument. This result then validates the extensive time and experimentation taken to get the older mass spectrometer into working order. 


\section{CHAPTER 3 : ANALYSIS OF PHOSPHOCHOLINES USING METASTABLE ATOM ACTIVATED DISSOCIATION MASS SPECTROMETRY (MAD-MS) AND COLLSION INDUCED DISSOCIATION (CID)}

\subsection{Introduction}

Recent advances in mass spectrometry have allowed scientists to probe different biological systems that were unattainable in the past. This increase in investigative power has contributed to the development of different fields of research including proteomics, ${ }^{179}$ genomics, ${ }^{180}$ and lipidomics. ${ }^{124,181}$ Lipidomics is defined as the full characterization of lipid molecular species and of their biological roles with respect to expression of proteins involved in lipid metabolism and function, including gene regulation. ${ }^{125}$ This field is expansive and covers everything from identifying which lipids are localized in cells to the role those particular lipids plays in a metabolic cycle. ${ }^{124}$ In the human body, these lipids have several functions such as determining cell membrane permeability ${ }^{182}$ and being part of the myelin sheath surrounding nerves. ${ }^{183}$ To fulfill these different roles lipids are very diverse in structure and chemistry and come in several different classes including sphingolipids, glycerolipids, and sterol lipids. These classes can then be broken down even further into subclasses. For example, glycerolipids include phospholipids like phosphotidylcholines, phosphotidylserines, and phosphotidylinositols. Because of this diversity in structure, biochemical researchers have typically studied one class of lipids at a time.

Mass spectrometry is an attractive technique for analyzing lipids due to its high selectivity and sensitivity, ability to quantify, abundance of structural information and ability to perform a variety of experiments. ${ }^{125}$ MS characterization of lipids began with electron ionization (EI) ${ }^{184-186}$ although most current researchers now rely on low-energy 
collisional induced dissociation (CID) $)^{70,71,187}$ for structural analysis ${ }^{131}$. The fragmentation products are normally restricted to $\left[\mathrm{M}+\mathrm{Y}-\mathrm{R}_{\mathrm{x}} \mathrm{CO}_{2} \mathrm{H}\right]^{+},\left[\mathrm{M}+\mathrm{Y}-\mathrm{R}_{\mathrm{x}} \mathrm{CHC}=\mathrm{O}\right]^{+}$, where X corresponds to the SN1 or SN2 fatty acid chain from which the fragment originated, and $\mathrm{Y}$ is the charging adduct, such as $\mathrm{Na}^{+}$or $\mathrm{H}^{+}$. The phosphocholine headgroup is another major fragment. ${ }^{71,188} \mathrm{CID}$ can provide useful information about a lipid by identifying its class, lipid chain lengths and the degree of unsaturation ${ }^{189}$ but this does not represent all of the pertinent information about the structure/function of lipids. The position and isomeric form of double bonds is also important. To understand the fragmentation mechanisms of lipids and the fatty acid chains, researchers are developing alternative fragmentation methods to CID including ETD $,{ }^{161} \mathrm{UVPD}^{190}{ }^{12} \mathrm{IRPD}^{190,}, 191$ and $\mathrm{MS}^{\mathrm{n}} .{ }^{71,192}$ These new methods have pros and cons and are generally used in tandem with conventional low-energy CID to get a more complete picture of the structure of the lipid in question. Additional approaches to tandem MS information include multistage mass spectrometry $^{72,189}$ and through the use of chemical derivatization. ${ }^{135-137,189}$ Blanksby's group has provided a straightforward and reliable method to determine the lipid class and double bond positions of unsaturated lipids using ozonolysis of gas-phase ions. ${ }^{135-137,189}$ An alternate tandem MS method currently under development is known as metastable atom activated dissociation (MAD). ${ }^{9,10,162-164,171}$ This technique has typically been limited to the fragmentation of peptides, but here we apply MAD to the fragmentation of targeted lipids using helium metastable atoms. In contrast to CID, which almost exclusively involves even electron rearrangements, MAD causes fragmentation through odd electron rearrangements, or radicals, that can induce fragmentation pathways unavailable through even electron mechanisms. 
This study aims to explore the fragmentation of five glycerophospholipids and one sphingolipid using MAD-MS to determine the dominant fragmentation pathways. Comparison of both MAD and CID experimental data will help to decipher those processes that are unique to MAD and its potential usefulness for future studies.

\subsection{Experimental}

\subsubsection{Instrumentation}

All experiments were performed on a modified EsquireLC or amaZon QIT mass spectrometer (Bruker Daltronics, Bremen, Germany), the former of which has been described in a previous work. ${ }^{9}$ Metastable atoms were generated with an Ion Tech FAB gun (P50, PSU, Teddington UK) and deflection electrodes were used to remove electrons and ions from the beam. The FAB gun was pulsed using custom electronics (described previously) to coincide with the fragmentation period in the scan function normally reserved for CID. The CID amplitude was set to $0 \mathrm{~V}$, so MAD would only occur during the typical fragmentation period. A visual schematic and description of the connections used in this process have been provided in a previous work. $^{9}$

\subsubsection{Reagents}

All the lipids used in this experiment were purchased from Avanti Polar Lipids (Alabaster, AL). Lipids used in this experiment included 1-hexadecanoyl-2-(9Z-octadecenoyl)sn-glycero-3-phosphocholine (POPC), 1,2-di-(9E-octadecenoyl)-sn-glycero-3-phosphocholine (9E DOPC), 1,2-di-(9Z-octadecenoyl)-sn-glycero-3-phosphocholine (9Z DOPC), 1,2-di-(5Z,8Z, 11Z, 14Z-eicosatetraenoyl)-sn-glycero-3-phosphocholine (DAPC), 1-palmitoyl-2-stearoyl-snglycero-3-phosphocholine (PSPC) and sphingomyelin. The HPLC grade methanol and glacial 
acetic acid were purchased from Sigma-Aldrich (St. Louis, MO). All lipids were reconstituted in a 9:1 mixture of methanol to water (with 1\% acetic acid). This provided lipid solutions that were approximately $60 \mu \mathrm{M}$ for analysis. Ultra high purity helium (Airgas, Parkersburg, WV) was used with the FAB gun and further purified using a noble gas purifier (HP2, VICI, Houston, TX) to remove impurities, which could otherwise prevent the formation of, or quench the metastable atoms.

\subsubsection{Method}

Singly charged lipid ions were generated through electrospray ionization (ESI) using an electronic syringe pump (74900, Cole-Parmer Instrument Company, Vernon Hills, IL) at a rate of $250 \mu \mathrm{L} / \mathrm{h}$. After injection, the sample was isolated using a width of $\mathrm{m} / \mathrm{z} 4$ before exposing them to the helium metastable atom beam at a low mass cut off ( $\mathrm{LMCO}$ ) of $\mathrm{m} / \mathrm{z} 100$. The metastable atom beam was typically pulsed on for $299 \mathrm{~ms}$ at an anode voltage of $6 \mathrm{kV}$. The vacuum chamber base pressure outside of the ion trap measured $1.68 \times 10^{-5} \mathrm{mbar}$ and was populated mostly by helium bath gas leaking out of the trap. After the addition of helium gas to the FAB gun, the vacuum pressure increased to $3.0 \times 10^{-5}$ mbar. The presence of a small gas load to the vacuum chamber from the FAB gun did not have any measurable effect on trap performance.

Collection of MAD data for each lipid took a total of 10 minutes and consisted of: 2 minutes of the full scan ESI spectrum of the sample, 2 minutes of the isolated precursor ion, 2 minutes of He MAD of the precursor with the deflection electrode on, 2 minutes of He MAD with the deflection electrode off, and 2 minutes of He MAD background signal (ESI off). These relatively long acquisition times enable us to average many spectra together to improve the signal-to-noise ratio of low mass peaks. The MAD background is defined as the ion signal that is 
collected with the FAB gun on and the ESI source off, thereby allowing us to characterize the Penning ionization (PI) background signal that is produced independently of the lipid precursor ions. We expect a mixture of ion/ion and metastable atom chemistry with the deflection electrodes off, but a higher relative proportion of metastable atom induced chemistry with the deflection electrodes on. MAD spectra shown in the figures have been background corrected using the average of the 2 minute FAB background signal.

The CID analysis was then performed following MAD acquisition and included: 2 minutes of the full-scan ESI spectrum, 2 minutes of the isolated precursor (no CID), 2 minutes of CID of the precursor and 2 minutes of background for a total analysis time of 8 minutes. Ion generation, accumulation, manipulation, and detection were optimized to provide consistent precursor ion signals of approximately $1 \times 10^{6}$ AU. All fragments were identified manually based on the predicted masses and were found to be within $\mathrm{m} / \mathrm{z} 0.6$ of the observed masses.

\subsubsection{CID}

All lipids were fragmented with the "SmartFrag" option in the Bruker EsquireLS NT 4.5

software. SmartFrag exposes the precursor ions to a linear amplitude modulated waveform from 30 to 200 percent of the selected CID amplitude. The fragmentation time was set to $25 \mathrm{~ms}$ in all the CID experiments. The amplitude setting of the CID ranged from 0.85 to $1.40 \mathrm{~V}$, depending on the mass that was being isolated. A typical acquisition spanning about 2 minutes contained approximately 400 spectra (each an average of 5 scans), which were subsequently averaged for data analysis. 


\subsection{MAD-MS of protonated phospholipids}

Initially, in this experiment we wanted to compare the fragmentation pattern induced by MAD to another more traditional fragmentation technique to investigate the novelty of MAD and determine if it could produce unique fragmentation compared to commonly utilized fragmentation methods. One of the most commonly utilized fragmentation techniques in mass spectrometry is CID. Figure 3. shows the comparison of the spectra obtained from the fragmentation of 9E DOPC using MAD and CID. In the CID spectrum, the major fragments that appear correspond to the phosphocholine headgroup and the complete loss of either fatty acid chain. The even electron, even mass losses of the fatty acid chains are typically referred to as the $\left[\mathrm{M}+\mathrm{H}-\mathrm{R}_{\mathrm{x}} \mathrm{CO}_{2} \mathrm{H}\right]^{+}$and $\left[\mathrm{M}+\mathrm{H}-\mathrm{R}_{\mathrm{x}} \mathrm{CHC}=\mathrm{O}\right]^{+}$fragments, where $\mathrm{X}$ corresponds to the $\mathrm{SN} 1$ or SN2 fatty acid chain from which the fragment originated. The CID fragmentation patterns observed for our lipids are consistent with previous studies obtained by other research groups studying low-energy CID of glycerophospholipids. ${ }^{70,71,187}$

When MAD and CID fragmentation is compared for 9E DOPC, MAD produces several CIDtype fragments too, like the $\left[\mathrm{M}+\mathrm{H}-\mathrm{R}_{\mathrm{x}} \mathrm{CO}_{2} \mathrm{H}\right]^{+}$at $m / z 506$ and $\left[\mathrm{M}+\mathrm{H}-\mathrm{R}_{\mathrm{x}} \mathrm{CHC}=\mathrm{O}\right]^{+}$at $m / z 522$. When MAD is performed on other lipids like POPC, 9Z DOPC and DAPC, they also show similar fragments, corresponding to the loss of the fatty acid chains. Scheme 3.1 shows an example of how a common CID fragment like the loss of the fatty acid chain could be achieved using MAD. In addition to producing fragments commonly observed in CID, MAD is also able to produce unique fragmentation. A series of fragment products are present in the $m / z 500$ to 800 range of Figure 3. that are not observed in the corresponding CID spectrum. Each member of this series is spaced in 14 unit increments that are normally indicative of the neutral loss of a $-\mathrm{CH}_{2}$ group from the target ion. The observation that there are so many of these products present in a 
row suggests that we may actually be observing the incremental fragmentation of the fatty acid in these lipids. This incremental fragmentation pattern is present in several of the phosphoglycerolipids studied including PSPC, POPC, 9Z DOPC, and 9E DOPC.

CID is not typically capable of producing fragmentation within the fatty acid chain of the lipid. ${ }^{72,189}$ Usually, $\mathrm{MS}^{\mathrm{n}}$ is required to achieve fragmentation within the lipid tail using CID. In fact, it was initially surprising that MAD could produce fragmentation throughout the entire fatty acid chain. The ability of MAD to cause fragmentation in the lipid was expected to be directed to areas where an electron rich group was present as is characteristic of metastable atoms inducing Penning ionization ${ }^{193,194}$ (Scheme 3.). The closest eligible atom to the fatty acid chain is the oxygen atom in the ester group at its head.

When Penning ionization occurs a radical and a charge would be generated on this oxygen group. The radical would only be able to move a few positions before inducing fragmentation. This preference during radical migration can be mainly attributed to energy required in each migration step. A radical normally migrates down a carbon chain through a series of 1,2 hydrogen rearrangements. ${ }^{195}$ These rearrangements are not as favored as other rearrangement configurations like a 1,4 hydrogen rearrangement and can cost up to $150 \mathrm{~kJ} / \mathrm{mol}$ of energy per move. ${ }^{195}$ The higher energy cost and reluctance of the compound to participate in many 1,2 rearrangements when other pathways are available generally causes a radical to induce fragmentation in an area close to where the radical was actually generated (Scheme 3.). This reluctance to migrate far from their point of generation makes it unlikely that the radical electron would travel to the end of the fatty acid chain to cause fragmentation An example of a fatty acid chain fragment that could be generated through short range radical migration instigated by 


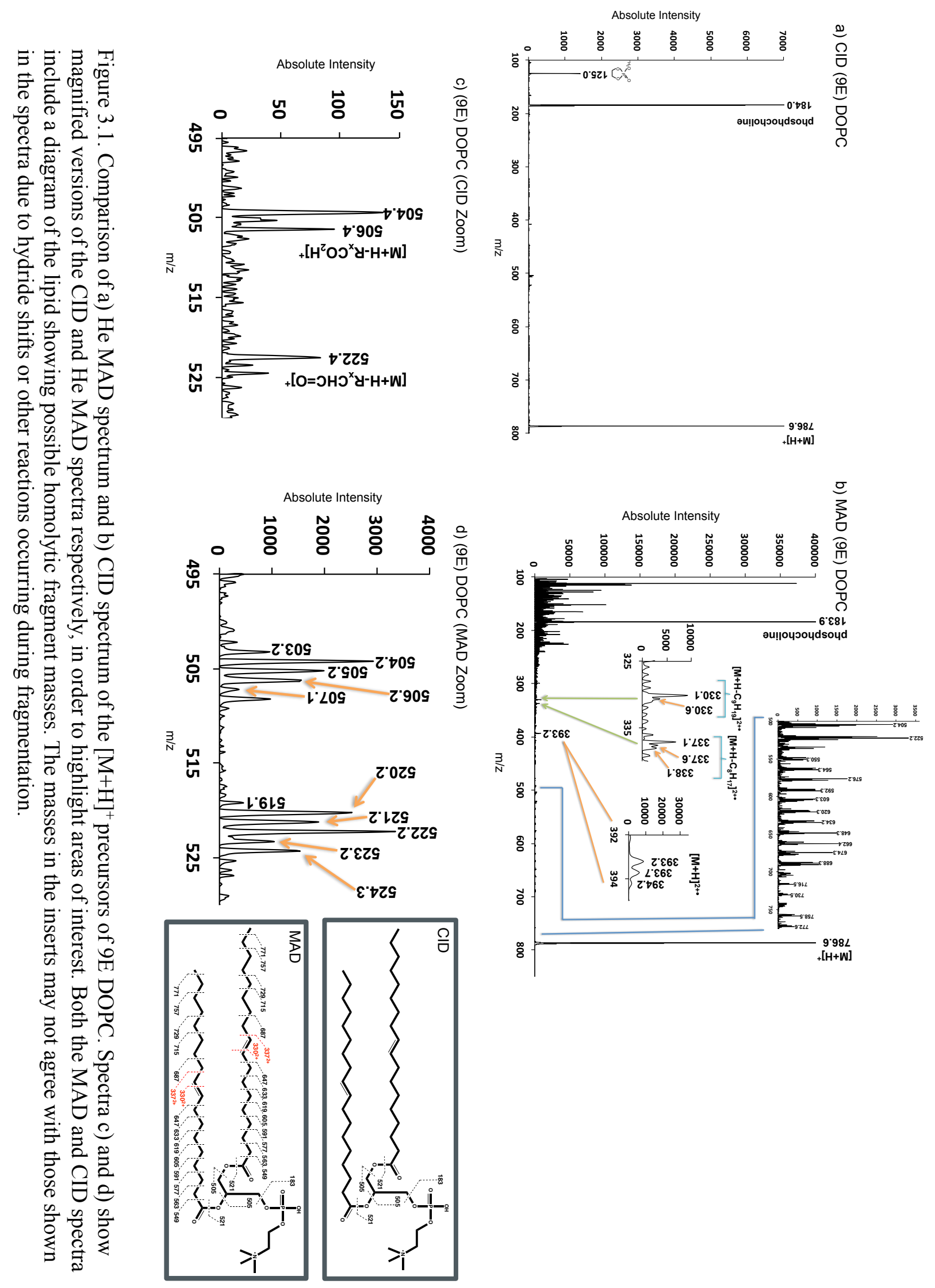




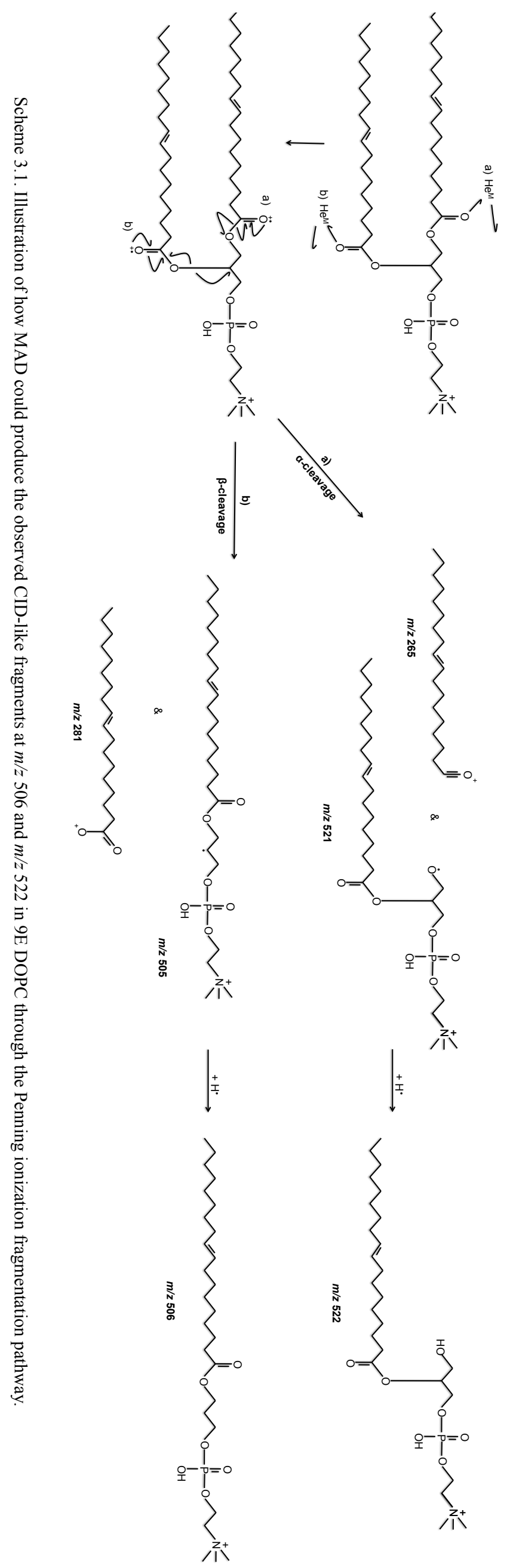


Penning ionization in PSPC is shown in Scheme 3.. To investigate this fragmentation phenomenon other fragmentation mechanisms observed for mass spectrometry in the literature were explored. One common fragmentation method associated with radical fragmentation is a McLafferty rearrangement. ${ }^{196}$ This mechanism is commonly associated with techniques like electron ionization (EI). In this mechanism the carbon chain will bend to allow the newly generated radical to attack a position further out on the chain from where it would normally be able to reach using radical migration. ${ }^{193}$ It is then possible that multiple McLafferty rearrangements could lead to the radical being passed to the end of the fatty acid chain. However, the fragmentation pattern produced by this rearrangement mechanism is not consistent with what we observe. This type of rearrangement would result in a staggered fragmentation pattern rather then the incremental pattern that we see. The staggered fragmentation pattern would be the result of the fragmentation being directed by the bending of the carbon chain during each rearrangement. Because of this difference in the expected fragmentation pattern it was necessary to identify another possible cause for the fragmentation pattern we were observing.

Another common mechanism associated with high-energy fragmentation processes is known as charge remote fragmentation (CRF). ${ }^{33,141-143,197} \mathrm{CRF}$ is commonly associated with fragmentation techniques like high-energy CID (HE-CID). CRF is a type of fragmentation that occurs away from a charge site and without any interaction between the reaction site and the charge site of the compound. ${ }^{141} \mathrm{CRF}$ occurs when significant internal energy is added to a target compound through an event like a high-energy collision. This internal energy briefly equilibrates over the entire compound before causing rearrangements to happen at the most labile points in accordance with RRKM theory. ${ }^{198}$ There are three major mechanisms that have been proposed for how rearrangements occur in CRF. The first method was proposed by Gross et. al. and 


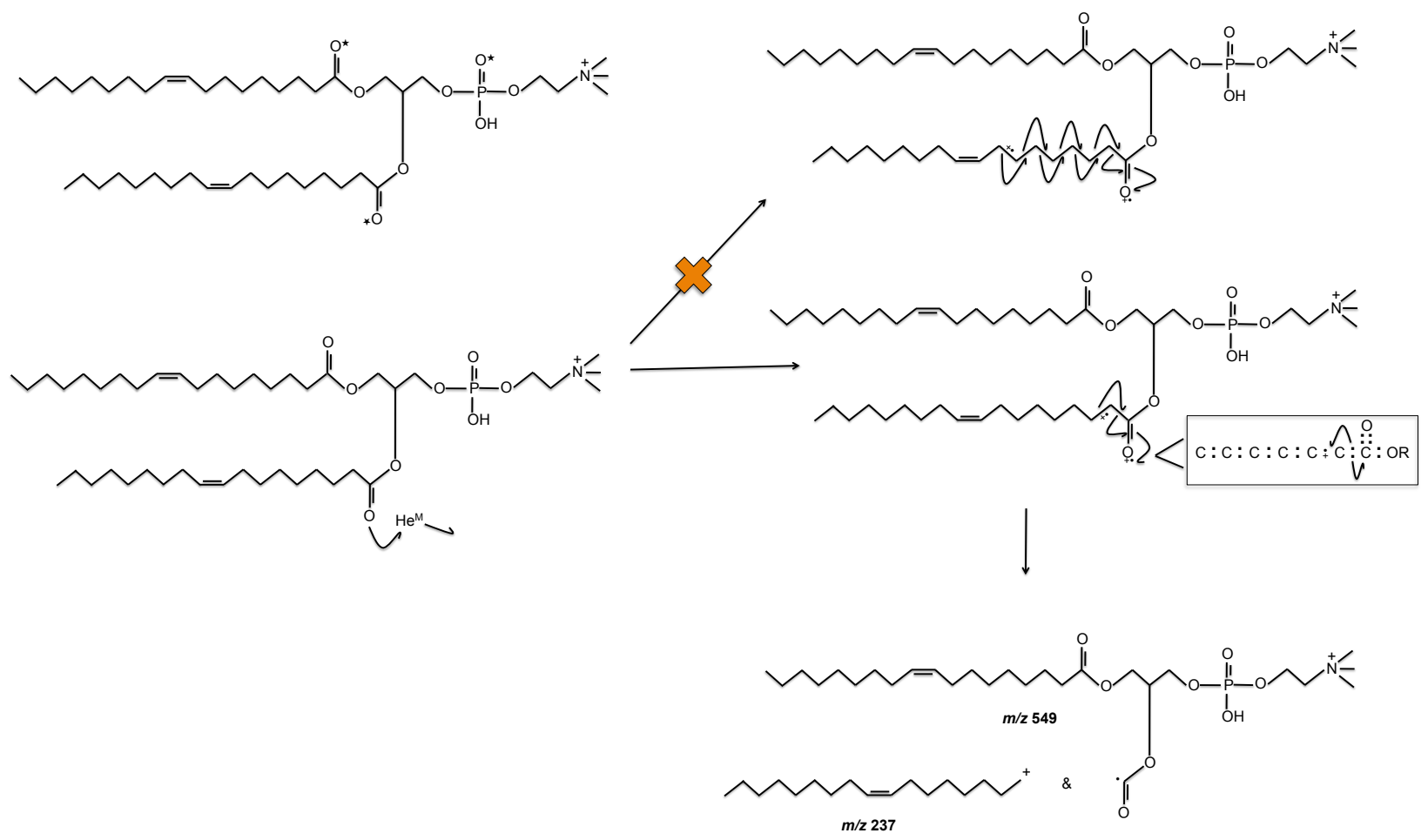

Scheme 3.2. An example of the radical fragmentation method that occurs in lipids after Penning ionization where a) $\star$ indicates the most likely points of attack by a metastable atom, and b) illustrates that radical migration is limited to only a few positions before causing fragmentation.

explains cleavages in terms of a 1,4 elimination reaction. ${ }^{141,197}$ Wysocki and Ross proposed a homolytic carbon cleavage and hydrogen rearrangement reaction as a way to explain some less abundant fragments normally associated with CRF. ${ }^{142}$ Lastly, Claeys et. al. suggested that a homolytic carbon-hydrogen cleavage may be another path to generate such fragments. ${ }^{143}$ By considering $\mathrm{CRF}$ as a possible fragmentation mechanism we are now entertaining the idea that MAD may be able to perform HE-CID-like fragmentation as an alternative pathway to Penning ionization. HE-CID is defined as a CID event that uses more then $1 \mathrm{keV}$ of energy. ${ }^{132}$ During MAD, metastable and ground state atoms are usually generated with up to $6 \mathrm{keV}$ of energy. So, the metastable atoms should plausibly retain at least the $1 \mathrm{keV}$ necessary to conduct HE-CID. The difference, is that here we would be accelerating the neutral atoms into the 
precursor ions whereas one normally accelerates the ions into the neutral atoms in HE-CID. Another factor to consider is that we are observing 14 unit mass separations between the product masses in our spectrum. The classic fragmentation pattern for CRF is a difference of $\mathrm{C}_{n} \mathrm{H}_{2 n+2}$ or 14 units for products of this mechanism. ${ }^{130}$ This classical pattern is similar to what we observe in our experimental results. When HE-CID was performed on protonated PC(18:0/18:1) by Shimma et. al., they found that aside from the typical low-energy CID fragments, they also produced a series of fragments associated with the fatty acid chain. ${ }^{42}$ A similar series of fatty acid fragments were also observed by Cheng and Gross when using high-energy CID to study triacylglycerols. ${ }^{130}$ In both instances the observed fragmentation was attributed to charge remote fragmentation. Both of these studies were performed using HE-CID and each produced a series of products similar to what was observed for POPC, 9E DOPC, 9Z DOPC, and PSPC in MAD (Figure 3.). The similarity between our results and those reported in the literature suggest that it is plausible that MAD could be performing HE-CID like fragmentation. This capability is interesting because HE-CID is normally performed in large expensive instruments like time-offlight (TOF) or Fourier transform ion cyclotron resonance (FT-ICR) mass spectrometers. A unique capability of MAD then may be its ability to produce these same fragments in a smaller, less expensive and more accessible instrument like an ion trap.

Aside from the fragments that we observe from the HE-CID like even electron rearrangement reactions, we also observe evidence that there is less dominant radical fragmentation occurring as well. Instead of the expected lone product peak at the mass corresponding to a cleavage in the fatty acid chain there is in actuality a group of products surrounding the expected fatty acid fragment of both even and odd mass (Figure 3.). In mass 


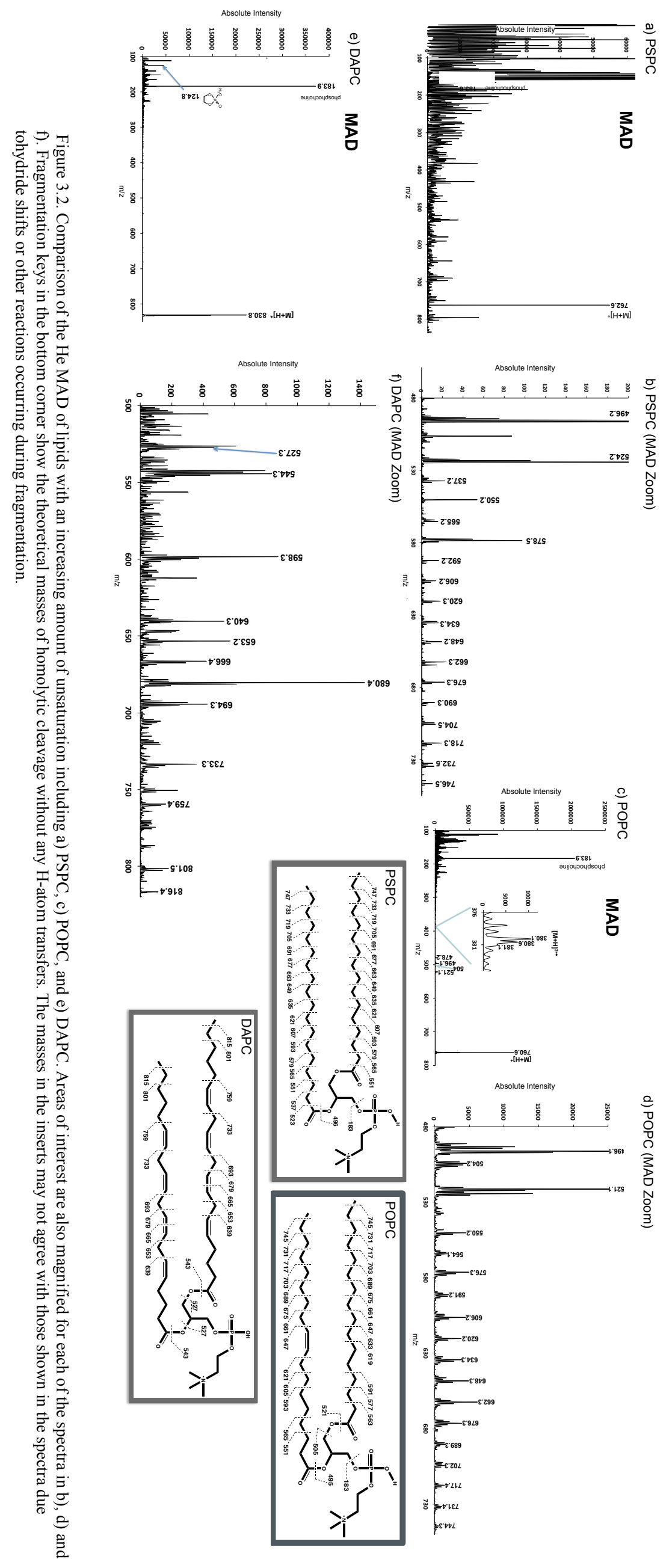




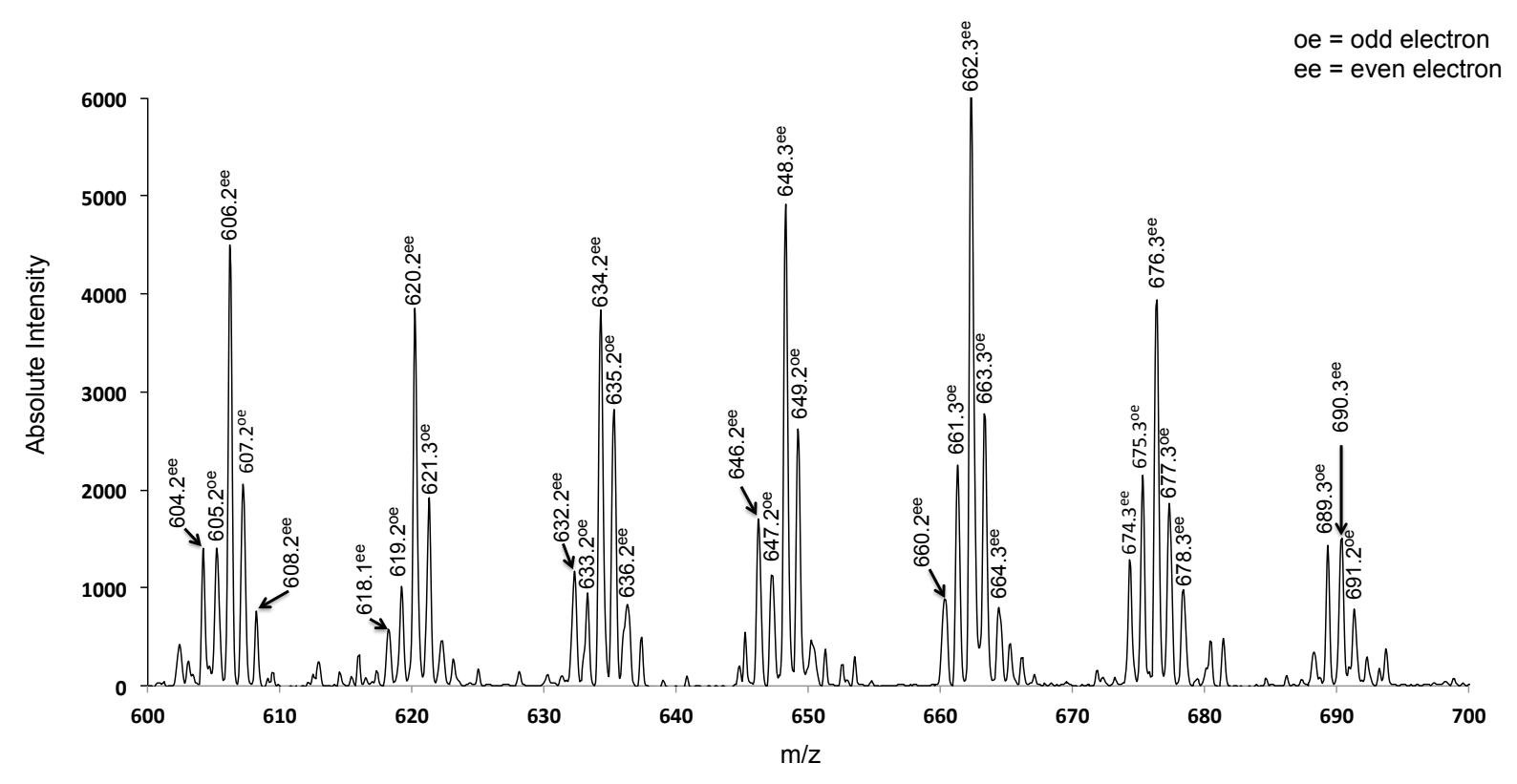

Figure 3.3. The four and five peak fragmentation patterns located within the charge-remotefragmentation-like fragmentation cascade occurring during He MAD of POPC.

spectrometry, when a compound has an odd number of nitrogen groups, a fragment that has an even mass has an even number of electrons whereas a fragment with an odd mass has an odd number of electrons. Because HE-CID utilizes even-electron rearrangements it will not produce odd mass products nor will it generate any additional charges during fragmentation. These odd masses could only be produced with the introduction of a radical electron. For example, in Figure 3.c the CID fragmentation shows even-electron products at $\mathrm{m} / \mathrm{z} 506$ and $\mathrm{m} / \mathrm{z} 522$. If we compare this to Figure 3.b and Figure 3.d we can see that the MAD spectra, displays these same products but also has other peaks corresponding to radical products not common in CID, like $\mathrm{m} / \mathrm{z}$ 505 and $m / z 521$ corresponding to $\left[\mathrm{M}+\mathrm{H}-\mathrm{R}_{\mathrm{x}} \mathrm{CO}_{2}\right]^{+\bullet}$ and $\left[\mathrm{M}+\mathrm{H}-\mathrm{R}_{\mathrm{x}} \mathrm{CCO}\right]^{+} \cdot$ respectively. These products are analogous to common $\beta$-cleavages observed in electron ionization of neutral esters. ${ }^{196}$ Another observation that suggests that MAD also undergoes radical mechanisms is the presence of the doubly charged fragment ions present in the spectrum of $9 \mathrm{E}$ DOPC and $9 \mathrm{Z}$ 
DOPC at $\mathrm{m} / \mathrm{z} 330, \mathrm{~m} / \mathrm{z} 337$ and $\mathrm{m} / \mathrm{z} 344$. Because the fragmentation process of CID does not allow for the generation of extra charges it is incapable of generating these $2+$ fragments through this mechanism. If the radical activation of the double bond were to follow typical electron ionization (EI) fragmentation mechanism then the most abundant products would be expected at both $m / z 323$ and $m / z$ 344. These cleavages are expected to be the most abundant because the radical electron would not have to move to cause fragmentation. These $2+$ fragments then taken in conjunction with the presence of the $[\mathrm{M}+\mathrm{H}]^{2+\bullet}$ product at $\mathrm{m} / \mathrm{z} 393$ indicates that radical fragmentation is likely occurring through the mechanism of Penning ionization (Eq. 14). An oxidized or Penning ionized precursor ion is unique to and commonly observed in MAD analysis of peptides and normally an indication that radical MAD fragmentation is occurring. ${ }^{9,163,164,171}$ The observation of Penning Ionized products is confirmed by the presence of the ${ }^{13} \mathrm{C}$ isotopomers at $m / z 0.5$ intervals from the ${ }^{12} \mathrm{C}$ monoisotopic ions.

$$
[M+H]^{+}+H e^{M} \rightarrow[M+H]^{2+\cdot}+H e^{O}+e^{-}
$$

In Figure 3.1 it appears that the doubly charged fragments are congregating around the masses associated with the location of the double bond in DOPC. This is interesting because these doubly charged fragments need a radical to form which means that a radical is making its way into the center of the fatty acid chain. One explanation for how this could occur is through the metastable attack of the fatty acid chain at the double bond. As stated before metastable atoms are attracted to areas of higher electron density. ${ }^{193,194}$ This attraction normally manifests as attacks on electron rich groups like oxygen or nitrogen lone pairs, followed by $\pi$-electrons in double bonds. Such preferential attack is based on dipole attraction and steric factors at close 
range. ${ }^{166,168,199,200}$ Thus, the non-bonding electrons of the oxygen ester groups and the $\pi$ electrons in the unsaturated lipids are potential sites for ionization. Scheme 3. illustrates a potential mechanism for how a $2+$ fragment could be produced as a result of this attack. The observation that metastable atoms will actively ionize double bonds is interesting for two reasons. First we see the less dominant radical fragment products occurring in the mass spectrum all around those peaks associated with the even electron products. By attacking the double bond MAD can induce radical dissociation further out in a carbon chain then it would normally be able to achieve using radical migration. Second, it may be possible to use this tendency to create $2+$ fragment ions around the double bond as a way to predict where the double bond is in the lipid. It is interesting to note that in the experimental data for 9Z DOPC in Figure 3.4 there is a group of

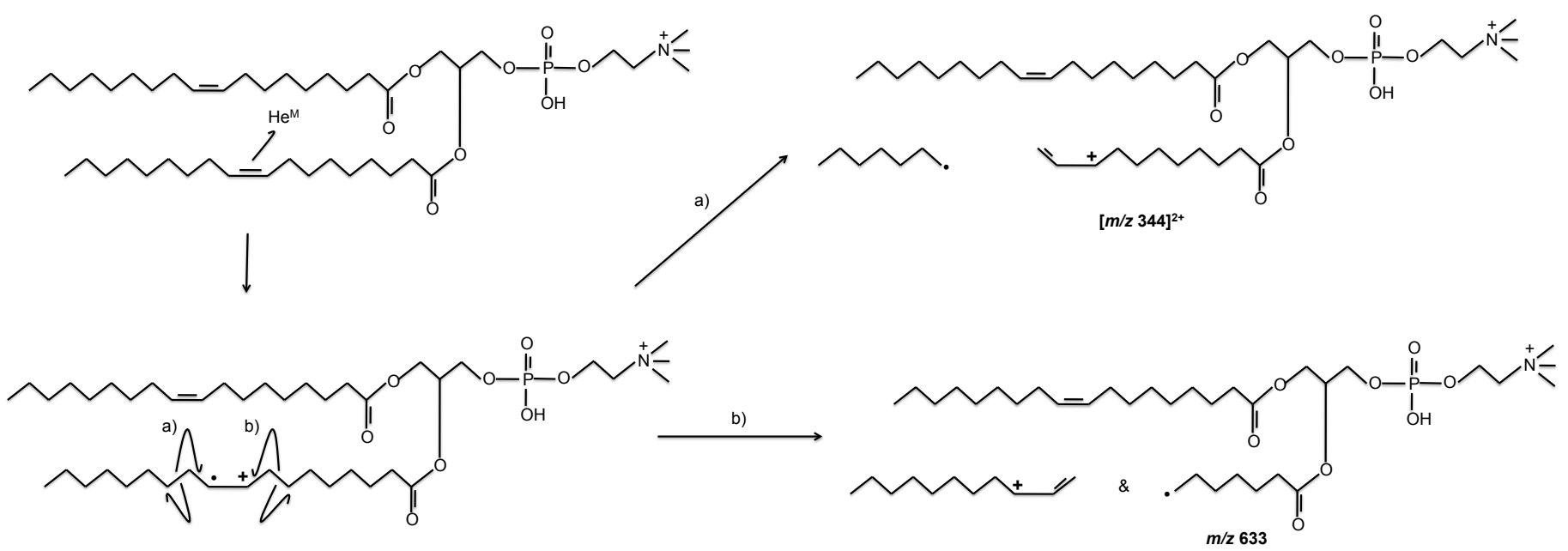

Scheme 3.3. An example of one doubly charged fragment formed from DOPC upon attack of the double bond by a metastable atom during MAD.

products at $\mathrm{m} / \mathrm{z} 632$ and $\mathrm{m} / \mathrm{z} 688$ that surround a third group of peaks centered around products of the double bond cleavage around $\mathrm{m} / \mathrm{z} 662$. The fragments at $\mathrm{m} / \mathrm{z} 632$ and $\mathrm{m} / \mathrm{z} 688$ correlate to 
McLafferty rearrangement products following Penning ionization of the double bond within the fatty acid chain. The high abundance of the products within these groups and the preference to undergo fragmentation at these bonds suggest that cleavage at these positions could be used to predict the location of the double bond. However, the spectra show a $2+$ product at $\mathrm{m} / \mathrm{z} 330$ as the most abundant doubly charged product. This product requires a hydride shift and cleavage across the double bond position to occur within the lipid before the fragments are formed which is difficult to explain using EI-type mechanisms. The product ions at 337 also require the loss of an even-electron neutral $\mathrm{C}_{8} \mathrm{H}_{16}$, to leave the $2+\bullet \quad$ radical product, which is also inconsistent with odd-electron rearrangements seen in EI of alkenes. Figure 3.4 also shows a decreasing trend in product abundance corresponding to the effective distance that the radical can move before it reacts. The further the radical needs to move to affect the bond the less likely it is going to interact with that bond decreasing the amount of times the bond is affected and the overall intensity of the product peak. The lack of doubly charged fragments on the side of the double bond closest to the headgroup is most likely due to increased background signal in the lower mass regions, which obscure the mass range where these products would appear (Figure 3.4). Admittedly, in this experiment we only observed the doubly charged fragments in the spectra of 9E DOPC and 9Z DOPC. POPC does not produce 2+ fragments even though one of the lipid tails has a double bond in the same position as DOPC. Presumably, the second double bond must either be involved with stabilizing the $2+$ ion, or else simply provide an additional site for attack and therefore higher probability of better signal-to-noise. Further experimentation would need to be performed to determine what factors mitigate when these $2+$ fragments are created and if their 
a) (9Z) DOPC

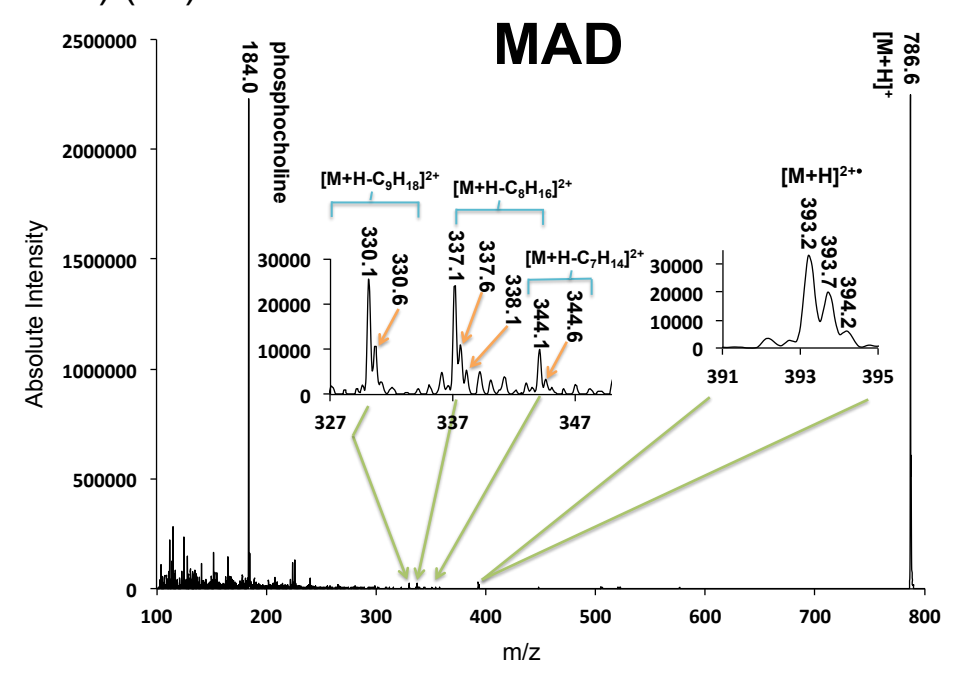

b) (9Z) DOPC

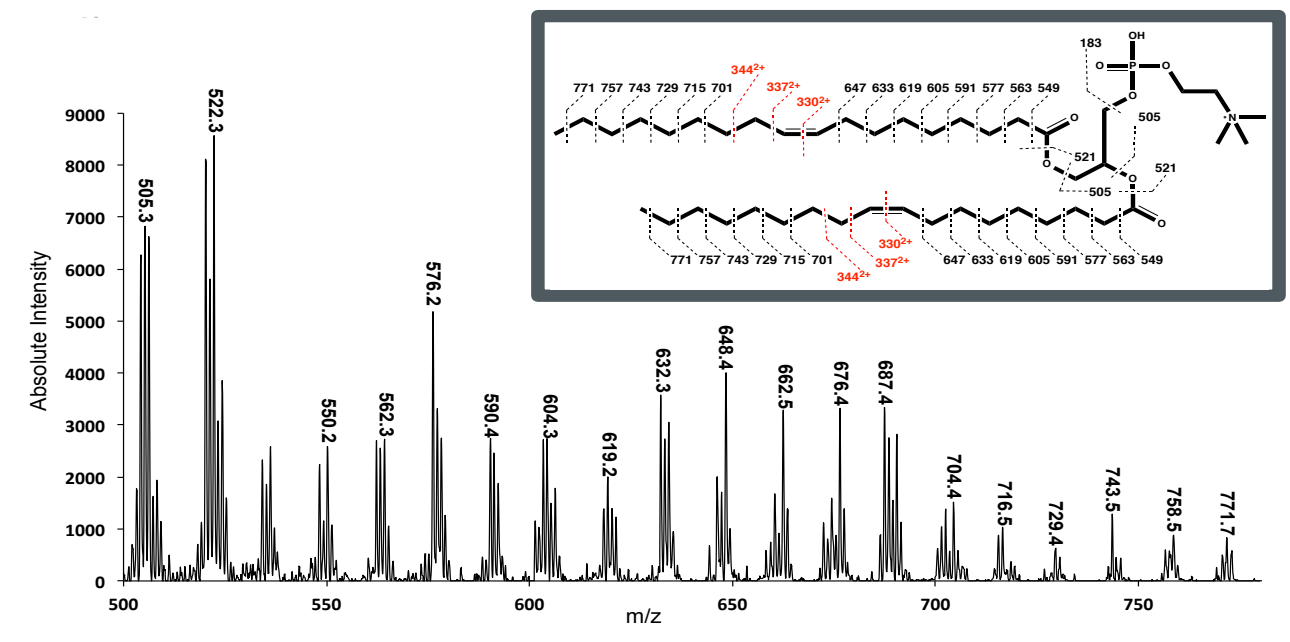

Figure 3.4. He MAD spectra of a) protonated (9Z) DOPC. Plot b is zoomed in on areas of interest in plot a. The inset shows possible cleavages and theoretical masses for homolytic fragmentation with no H-atom transfers. The masses in the insert may not agree with those shown in the spectra due to hydride shifts or other reactions occurring during fragmentation.

location is solely linked to the location of the double bond and not another unknown determinant.

As mentioned earlier, MAD has been used successfully in the past to fragment peptides in a way that was dominated by radical fragmentation and produced unique products compared 
to other techniques like CID, ETD or ECD. Although MAD does produce some unique products, MAD fragmentation of lipids appears to resemble HE-CID. One reason for this is that the structure of lipids and peptides are very different. In peptides, there are more electron rich groups in the amide backbone that make Penning ionization more probable. In lipids, Penning ionization is more probable in the headgroup or at double bonds in the fatty acid chain and results in fewer places for metastable attack. Once the fatty acid chain is activated with a charge and a radical, rapid scrambling and hydride shifts lead to almost random cleavage, as observed in HE-CID. Up to this point we have discussed lipids that fragment very effectively in both MAD and CID. However, there were two lipids analyzed as part of this study that did not as completely as the other lipids described. These two lipids were sphingomyelin and DAPC.

In contrast to the fragmentation patterns for the lipids described above, when CID is performed on sphingomyelin, it typically produces the charged phosphocholine headgroup fragment and no other significant fragments. ${ }^{69,177,178}$ The results of the MAD fragmentation of sphingomyelin are very similar to those of the in-house CID experiment with the phosphocholine fragment being the predominant product (Figure 3.). Small neutral losses are also apparent in the in-house CID spectrum of sphingomyelin such as water loss at $m / z 713$ and the formation of the $\mathrm{O}, \mathrm{O}^{\prime}$-dimethylenephosphoric acid, at $\mathrm{m} / \mathrm{z}$ 124. In this case it is possible that the O,O'dimethylenephosphoric acid at $m / z 124$ was also formed in the MAD spectrum as well but was overwhelmed by the background signal. In a typical MAD analysis background signal between $\mathrm{m} / \mathrm{z} 100$ and $\mathrm{m} / \mathrm{z} 300$ is present due to the Penning Ionization of residual pump oil in the ion trap. DAPC is less reluctant then sphingomyelin to fragment although it produces less fragmentation overall then the other lipids studied in this experiment. Whereas lipids like POPC, PSPC, 9E DOPC, or 9Z DOPC would produce fragmentation at almost every point in the fatty acid chain 
a) Sphingomyelin

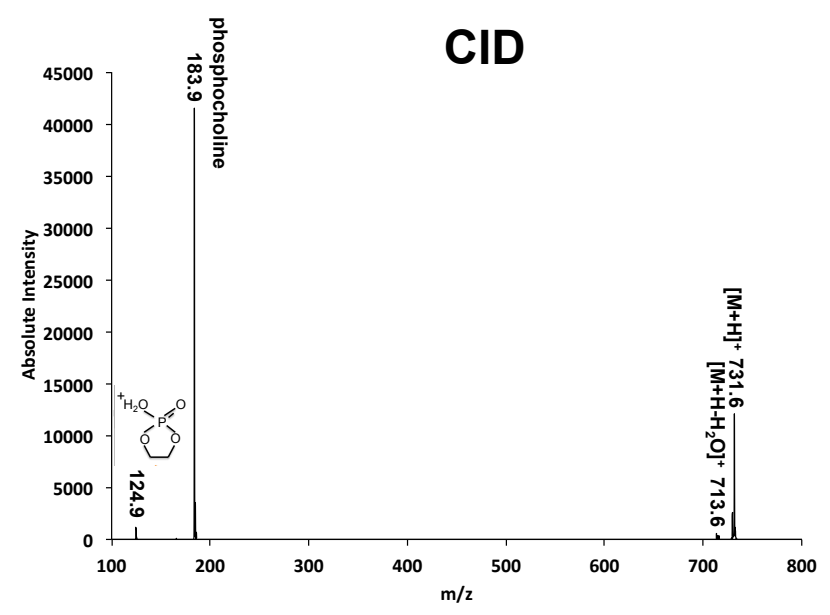

b) Sphingomyelin

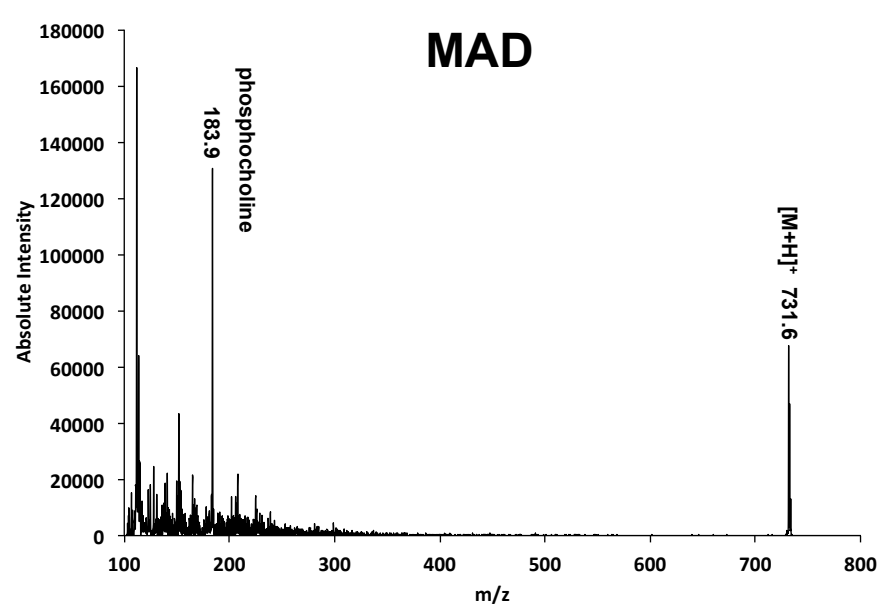

Figure 3.5. Comparison of the a) CID spectrum and b) He MAD spectrum of the $[\mathrm{M}+\mathrm{H}]^{+}$form of 18:0 sphingomyelin (SM).

DAPC would instead have more selective fragmentation. Scheme 3.1 shows an example of how some of fragments we observe in DAPC could be formed. The less abundant fragmentation that we are observing in DAPC can mostly likely be attributed to the number of double bonds present in the lipid. DAPC has eight double bonds in its structure, which is several more then the next closest lipid analyzed in the experiment, which was 9E DOPC or 9Z DOPC which both had two. These additional points of unsaturation could provide extra pathways for stabilization during fragmentation through the use of resonant structures. In this way, DAPC may be able to adjust the double bonds in its fatty acid chain to compensate for some of the energy present during fragmentation and prevent fragmentation from occurring at locations where it would be unavoidable in other lipids that do not have this capability. The capability of chemical compounds to use resonant structures for stabilization is well established to the point that some researchers focus exclusively on exploring this concept. ${ }^{201-203}$ One other product that we notice is 
missing from DAPC and sphingomyelin is the Penning ionized peak. Although the intact Penning ionized products appear in the spectra for POPC, 9E DOPC, and 9Z DOPC, they are not observable in the spectra of DAPC or sphingomyelin. This lack of a Penning ionized peak in DAPC and sphingomyelin could be attributed to a combination of the ionization energy of the $[\mathrm{M}+\mathrm{H}]^{+}$precursor ion, competition between electronic and vibrational excitation, and the relative lability of the $[\mathrm{M}+\mathrm{H}]^{2+\bullet}$ product ions. Although a Penning ionized $[\mathrm{M}+\mathrm{H}]^{2+\bullet}$ is not observed in DAPC, there are many odd-electron fragment ions that are indicative of Penning ionization induced dissociation. For example, the fragmentation spectra of DAPC in Figure 3.2e and Figure $3.2 \mathrm{f}$ shows packets of peaks at centered at $\mathrm{m} / \mathrm{z} 543$ and $\mathrm{m} / \mathrm{z} 694$ which include both even and odd<smiles>CCCCC/C=C\CC/C=C\CCCC(=O)OCC/C=C\CCCCCC</smiles>

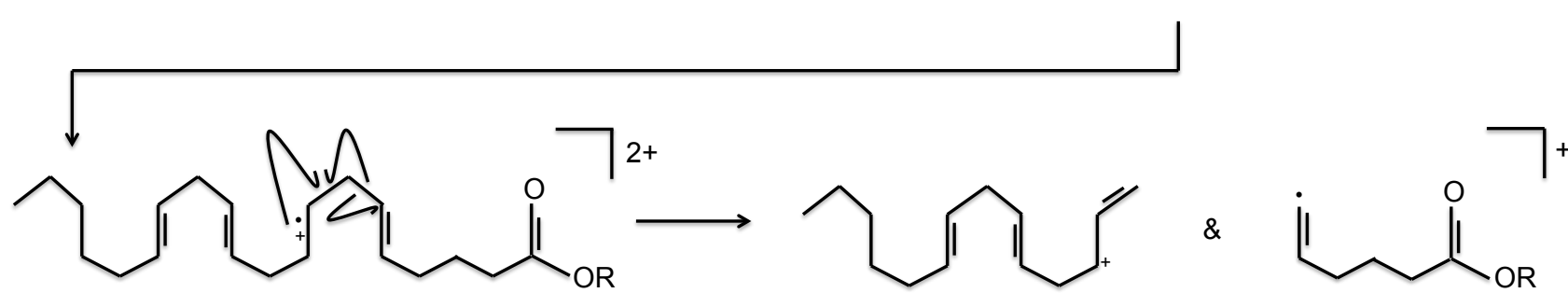
$m / z 191$

$m / z 639$

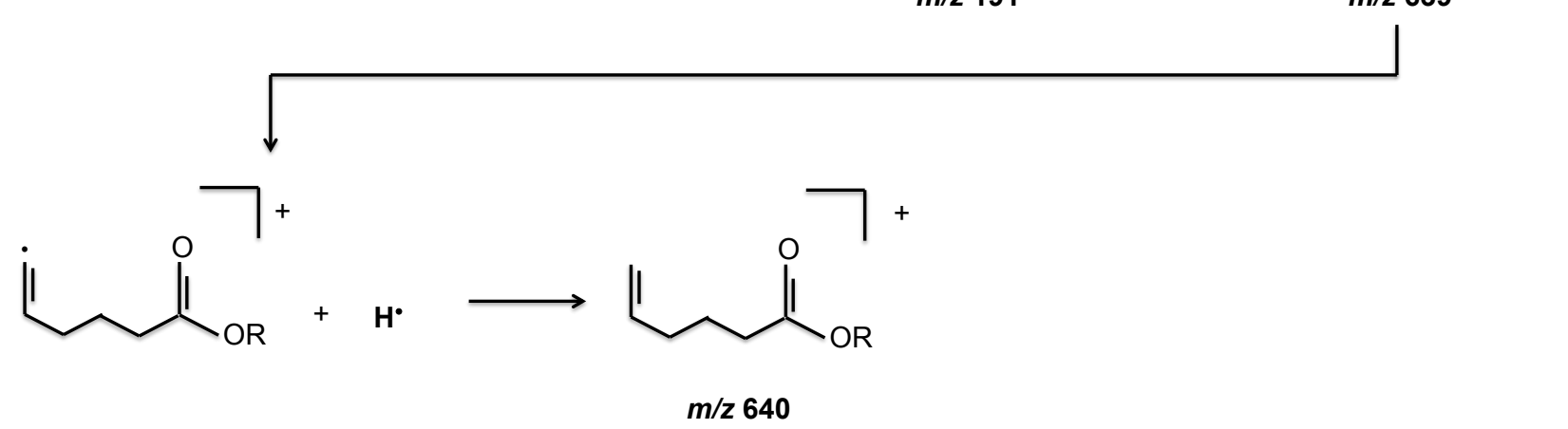

Scheme 3.4. Illustration of the radical fragmentation path instigated by Penning ionization which could occur in a highly unsaturated lipid like DAPC. 
electron products. The presence of odd electron products is not common in HE-CID of lipids ${ }^{24,25}$, 42,32 and thus can be attributed to radical fragmentation originating from Penning ionization, despite the lack of a visible $[\mathrm{M}+\mathrm{H}]^{2+\bullet}$ product.

Through the analysis of the six lipids in this experiment using both MAD and CID it was determined that MAD could effectively provide those fragments normally produced during CID. Aside from the production of common CID products MAD was also able to produce fragments normally associated with HE-CID as well as unique radical products closely associated with the ability of MAD to induce Penning ionization.

\subsection{Conclusion}

MAD-MS was used to study six different lipids in the protonated form to determine the fragmentation patterns that could be achieved using this new fragmentation method with this type of compound. CID was then used as a basis of comparison. MAD-MS was able to produce the phosphocholine headgroup, $\left[\mathrm{M}+\mathrm{H}-\mathrm{R}_{\mathrm{x}} \mathrm{CO}_{2} \mathrm{H}\right]^{+}$and $\left[\mathrm{M}+\mathrm{H}-\mathrm{R}_{\mathrm{x}} \mathrm{CHC}=\mathrm{O}\right]^{+}$fragments typically associated with the CID of the lipids tested but also produces odd-electron species such as odd mass products in the fragmentation series in POPC, PSPC, 9E DOPC, and 9Z DOPC. The $2+$ fragments in 9E DOPC and 9Z DOPC also presented evidence that radical mechanism were occurring alongside the even electron rearrangement and was unique to MAD indicating fragmentation at or around the double bond. MAD yielded fragments associated with both radical induced and HE-CID fragmentation pathways within the fatty acid chain of PSPC, POPC, 9E DOPC and 9Z DOPC. Despite their presence the radical products were dominated by those produced from the even electron rearrangements. Although, MAD appears to be as effective as CID for analyzing these six lipids MAD was unable to produce significantly more information then can be easily obtained using $\mathrm{MS}^{\mathrm{n}}$, CID or HE-CID. MAD then appears to have the ability to 
produce high-energy CID fragments in compounds where Penning ionization is not easily accessible allowing a high energy CID analog to be performed in an ion trap. Further experimentation with a larger pool of lipids will help to decipher the nature of this fragmentation pathway. 


\section{CHAPTER 4 : DIRECT ANALYSIS OF DRUGS IN FORENSIC MEDIA USING LASER ABLATION ELECTROSPRAY IONIZATION MASS SPECTROMETRY (LAESI-MS).}

\subsection{Introduction}

In 1970, the US government passed into law the Controlled Substances Act, which helped to identify and rank substances of abuse and provide a scale of punishments associated with the manufacture, sale and use of such substances. The list of scheduled drugs is regularly updated at the state and federal levels. Despite these controls, drug abuse is a very common crime and drug analyses comprise the major workload of most forensic laboratories. ${ }^{204}$ As a result, many laboratories have large backlogs of evidence awaiting analysis. New, faster methods of confirmation are necessary to help laboratories keep pace with evidence submissions.

Typically, the analysis of suspected drugs is performed using a sequence of increasingly selective tests. The first test is usually a presumptive test and includes polarized light microscopy, immunoassays or color tests. ${ }^{205,206}$ Presumptive tests are used to determine which samples are most likely to contain an illicit substance and to help identify the general class of the drug or drugs present. These tests guide the conditions for subsequent methods of instrumental analysis. Presumptive tests are commonly utilized because they are easy to perform and interpret in the field and do not require much training or chemical expertise. However, positive results in this type of test are usually not specific enough to confirm the presence of a particular drug, which is why seized samples are always sent to a crime laboratory for confirmatory tests. Currently, the most common confirmatory tests are Gas Chromatography/Mass Spectrometry (GC/MS) and Fourier transform infrared spectroscopy (FTIR) although High Performance Liquid Chromatography Mass Spectrometry (HPLC/MS) is also gaining popularity in toxicology laboratories. ${ }^{207}$ These techniques have become widespread due to their sensitivity, ability to 
identify multiple components at once, and their ability to be easily automated. However, a typical GC or LC run is 20 to 30 minutes long, not including sample preparation. This time requirement is a major reason for backlogs. Fast $\mathrm{GC}^{208-211}$ is a potential approach for decreasing analysis times, but this approach does not reduce sample preparation time. Because of these potential drawbacks, new instrumental methods of analysis are being investigated to complement current techniques. Mass spectrometric methods of analysis that are gaining interest in various forensic laboratories are ambient ionization techniques like Direct Analysis in Real Time $(\mathrm{DART})^{75}$ and Desorption Electrospray Ionization (DESI). ${ }^{84}$

DESI was introduced in 2004 by Cooks and co-workers at Purdue.$^{84}$ DESI is a technique that can analyze samples under ambient conditions. In DESI, an ESI plume is directed at a sample surface and the desorbed droplets are then sampled by the mass spectrometer. Common surfaces used for DESI include those made of polytetrafluorethylene (PTFE) for negative mode analysis, and polymethylmethacrylate (PMMA) for positive mode analysis, although almost any non-conductive surface can work. ${ }^{77,85}$ Because the final multiply-charged products of DESI are essentially the same as from an ESI source, any mass spectrometer that can operate with an atmospheric pressure ionization source (API), like ESI, will work with DESI. ${ }^{77}$ DESI has been coupled with a variety of mass spectrometers including ion traps, ${ }^{77}$ Orbitraps,${ }^{86}$ Fourier transform ion cyclotron resonance (FT-ICR), ${ }^{87}$ and ion mobility time of flight (IMS-TOF) $)^{212}$ mass spectrometers. DESI has the ability to analyze molecules up to 66,000 Daltons. ${ }^{77,89}$ DESI has been used in a variety of forensic applications including detection of illicit drugs, ${ }^{90-92}$ explosives, ${ }^{93-95}$ alkaloids in plant matter ${ }^{213}$ and imaging and analysis of latent fingerprints. ${ }^{96}$ DART was first reported in 2005 by Cody and Laramee after trying to develop an atmospheric pressure thermal electron source. ${ }^{75}$ DART normally forms singly charged, 
protonated or deprotonated species in either the positive or negative mode, respectively, but can form radical ions in some cases. ${ }^{76}$ Ionization in DART normally occurs either through proton transfer, Penning ionization or electron capture. ${ }^{78}$ Unlike DESI, DART is typically limited to analytes with a molecular weight below 800 Daltons ${ }^{76,77,79,80}$. Since its inception, DART has also been used for a variety of forensic applications including chemical warfare agents ${ }^{75}$, explosives, ${ }^{81}$ drugs, ${ }^{82,83,214}$ and ignitable liquids. ${ }^{75}$

There are now dozens of variations of ambient ion sources with different merits. ${ }^{77,97,} 215$ LAESI, which was first presented by Nemes and Vertes in 2007, addresses some of the problems associated with other ambient ionization techniques, such as the requirement of an external matrix in matrix assisted laser desorption electrospray ionization (MALDESI), an ill-defined surface area (DESI), or a limited mass range (DART). ${ }^{98}$ LAESI functions by illuminating the sample with a pulsed mid-infrared Er:YAG laser that is tuned with an optical parametric oscillator (OPO) crystal to $2940 \mathrm{~nm}$, which matches the vibrational state of the $\mathrm{OH}$ bonds in water. ${ }^{34}$ Effectively, the laser energy is rapidly absorbed by the water in a sample, leading to vaporization and the formation of an ablation plume above the sample surface. The ablated sample particles intersect with an electrospray plume located above the stage, wherein ablated molecules are ionized and carried into the mass spectrometer for analysis. Because ablation relies on the excitation of $-\mathrm{OH}$ bonds, the sample matrix must contain at least one major component with $-\mathrm{OH}$ bonds, such as water or methanol.

By using conventional ESI-based ionization, LAESI-MS also enables an extended mass range and can analyze samples up to 66,000 Daltons. ${ }^{98}$ An additional advantage of LAESI-MS over other techniques is its ability to perform high throughput automated analyses of liquid samples. Example applications of LAESI-MS are molecular imaging, ${ }^{99}$ imaging of metabolites 
in plants, ${ }^{100}$ and rat brain tissue sections. ${ }^{100}$ Direct studies have also been performed using LAESI-MS on cyanobacteria ${ }^{102}$ and individual plant cells. ${ }^{103}$

In addition to bulk drug samples described above, the forensic and criminal justice system is also concerned with the identification of drugs in biological fluids and other matrices. Many workplaces institute mandatory drug tests to assess employee adherence to company policy. The criminal justice system uses these types of tests to assess adherence to parole regulations or cause of death investigations. These drug tests are normally performed with the urine of living individuals or the blood of deceased individuals, mostly due to ease of collection. A challenge associated with urine samples is that in order to respect the privacy of the donor, the urine is normally collected without direct supervision and donors have been known to tamper with the sample through the use of surrogate urine bags or adulterants like bleach or vinegar. ${ }^{216 \text {, }}$ ${ }^{217}$ Because of these challenges, interest has focused on implementation of these tests using other types of biological samples, such as saliva or human hair, which can be collected with direct supervision. Hair samples have a major advantage of storing a chronological record of past drug use. Hair samples are also more difficult to falsify or contaminate and are easy to collect and store. Despite these advantages, institutions have been slow to rely on hair testing because of the lengthy extraction procedures that are required to efficiently remove, concentrate, derivatize, separate and detect analytes in hair.

In this work we seek to expand the application of LAESI-MS into the realm of forensic science through the analysis of controlled drugs in powder samples, plant matter and human hair. These experiments demonstrate the feasibility of LAESI-MS as an alternative confirmatory (category A) ${ }^{206}$ technique for the detection of drugs of abuse. We will show that common drugs 
of abuse can be detected quickly and accurately in a variety of media and with minimal sample preparation (wetting).

\subsection{Experimental}

\subsubsection{Reagents}

All drugs utilized in this experiment were purchased from Sigma-Aldrich (St. Louis, MO). The cannabis leaves were obtained from Ohio Bureau of Criminal Investigation (BCI) in London, Ohio. The deuterated methamphetamine standard (Cat\# M-093) was obtained from Cerilliant (Round Rock, TX). The drug-laced hair standards (Drugs of Abuse in Human Hair Segments, SRM \# 8448 and Drugs of Abuse in Human Hair II, SRM\# 2380) were purchased from the National Institute of Standards and Technology (Gaithersburg, MD). The Mandelin, Marquis, Dille-Koppanyi and Cobalt Thiocyanate reagents were prepared in house ${ }^{205}$ and required cobalt thiocyanate, cobalt acetate dehydrate, and ammonium vanadate which came from Sigma-Aldrich (St. Louis, MO) as well as methanol, glacial acetic acid, isopropylamine, sulfuric acid and $40 \%$ formaldehyde which were purchased from Fisher Scientific (Hampton, NH). The hydrochloric acid and sodium hydroxide used were also purchased from Fisher Scientific (Hampton, NH).

\subsubsection{Instrumentation}

Experiments were performed using a Protea LAESI DP-1000 Direct Ionization System (Protea Biosciences, Morgantown, WV) attached to a Velos linear ion trap (LTQ) mass spectrometer (ThermoFisher Scientific, Waltham, MA). For all analyses described, ablation was carried out with a $2940 \mathrm{~nm}$ infrared laser operating in pulsed mode at $10 \mathrm{~Hz}$. Drugs of abuse were analyzed from 96 well plates with 100 laser pulses per sample and with a delay of 4 
seconds between wells. For the analysis of hair and cannabis leaves, 10 pulses were used from the same $10 \mathrm{~Hz}$ laser at $\sim 200 \mu \mathrm{m}$ to $500 \mu \mathrm{m}$ intervals along the hair in a grid-like raster resulting in 280 to 900 analysis locations (pixels) per sample. The ESI spray solution consisted of a $0.1 \%$ acetic acid solution in 50\% methanol flowing at a rate of $1 \mu \mathrm{L} / \mathrm{min}$. Each analysis utilized tandem mass spectrometry with the dynamic exclusion mode enabled. In this mode the mass spectrometer searches the spectrum for the most abundant ion, isolates and performs CID on the most abundant peak. The mass spectrometer then finds the next most abundant peak, excluding the previous precursor ion. In this way the mass spectrometer identifies and analyzes several of the most abundant ions in a well at a particular time. A 96 well plate can be analyzed as quickly as $\sim 8$ min for full-scan MS mode and in $\sim 30$ min using dynamic exclusion MS/MS mode. The MS/MS mode can be used to identify drugs in solution at an average rate of 20 seconds per sample. An analysis time of $\sim 30$ minutes was required to generate an ion map of the plant leaf, but this varied based on the number of pixels required to fully map the leaf surface. Illustrations of the components of this system have been covered in elsewhere. ${ }^{98,218}$

\subsubsection{Method}

Experiments herein focused on the detection of drugs in three different types of media using LAESI-MS. The first analysis performed was on drugs that had been dissolved in different reagent solutions. The drugs used for this experiment included cocaine, methamphetamine, amphetamine, phenobarbital, oxycodone, codeine and quinine - a common cutting agent. Solutions of each drug ranging from $0.01 \mathrm{mg} / \mathrm{mL}$ to $5 \mathrm{mg} / \mathrm{mL}$ were prepared from solid drug samples. Each drug was then dissolved in a variety of solvents before analysis, including water, 0.1 M hydrochloric acid, 0.1 M sodium hydroxide, and each color reagent (Scott's cobalt 
thiocyanate reagent, Marquis reagent, Mandelin reagent, and Dille-Koppanyi reagent) ${ }^{205} \mathrm{We}$ selected these specific color reagents because they are among the more commonly used color test reagents, and because they span a range of $\mathrm{pH}$ values and organic content. These combinations were then placed in random order into six 96-well-plates. Two 50/50 mixtures of cocaine/quinine and cocaine/aspirin were also tested at a variety of concentrations. In summary, the color tests included a total of 252 solutions: nine different drug/drug combinations at four different concentrations, each in seven different reagents. One well plate also contained a series of methamphetamine samples ranging from 1 to $100 \mathrm{ppm}$, which were used to test the dynamic and linear ranges of the instrument. Samples were tested immediately after preparation. We have not yet tested the reliability of repeated freeze-thaw cycles on the analytical results but this could be an important factor for implementation into laboratories with respect to long-term storage of samples.

The second type of matrix or media was human hair. This experiment was performed by first attaching several strands of hair to a microscope slide using removable, double-sided Scotch tape (3M). These hairs were then wetted with water and allowed to sit for a few seconds to absorb the water. The sample was then analyzed directly using LAESI. An ion map was generated using ProteaPlot software (Protea Biosciences Inc., Morgantown, WV) from the raw XCalibur files to show the abundance of the different drugs distributed across the group of hair strands attached to the sample slide.

The cannabis leaves were analyzed primarily to identify the presence of the psychoactive ingredient, tetrahydrocannabinol (THC), and secondarily to image the distribution of THC throughout the leaf. Different methods of attaching the leaf to the slide were tested to determine if any excess background signal was generated in LAESI from the different adhesive media. The 
leaf was attached to a microscope slide using either paraffin wax or Permount solution. After mounting, the leaf was then wetted using water and allowed to sit for several minutes before being placed into the instrument and analyzed directly using LAESI. An ion map was generated from the raw data file using ProteaPlot software.

\subsection{Results and Discussion}

\subsubsection{Analysis of drugs in solution after a presumptive color test}

To prepare the well plates for analysis using LAESI-MS, different combinations of drugs and reagents were added to each well, as described in the method section. When some of the color reagents were combined with the drug, they reacted to produce a color indicative of the drug present. Some examples of color test results are shown in Figure 4.1, where the two blue wells near the left and center of the plate show a positive test for cocaine with the Scott reagent (cobalt thiocyanate). These two wells show the different degrees of color that could develop with different drug combinations. The red square in Figure 4.1 shows a mostly negative pink color result with a faint blue ring indicative of cocaine developing around the edge of the well. This well contained $2.5 \mathrm{mg} / \mathrm{mL}$ of cocaine and $2.5 \mathrm{mg} / \mathrm{mL}$ of aspirin. In contrast, the blue box shows a simple positive result for cocaine at $5 \mathrm{mg} / \mathrm{mL}$. After developing the color tests, the well plate was analyzed using LAESI-MS/MS.

Tandem MS data were compared with the NIST standard reference tandem MS spectra to confirm the presence of the expected drugs. Analyses were focused on the unreacted, unmodified drugs, even though some of the color tests, such as Marquis, are known to involve covalently modified products. An example of a positive identification between the experimental and reference data for methamphetamine is shown in Figure 4.2. These analyses found that the target 
drugs could be identified most successfully at concentrations of $1 \mathrm{mg} / \mathrm{mL}$ and $5 \mathrm{mg} / \mathrm{mL}$, as seen in Figure 4.3.

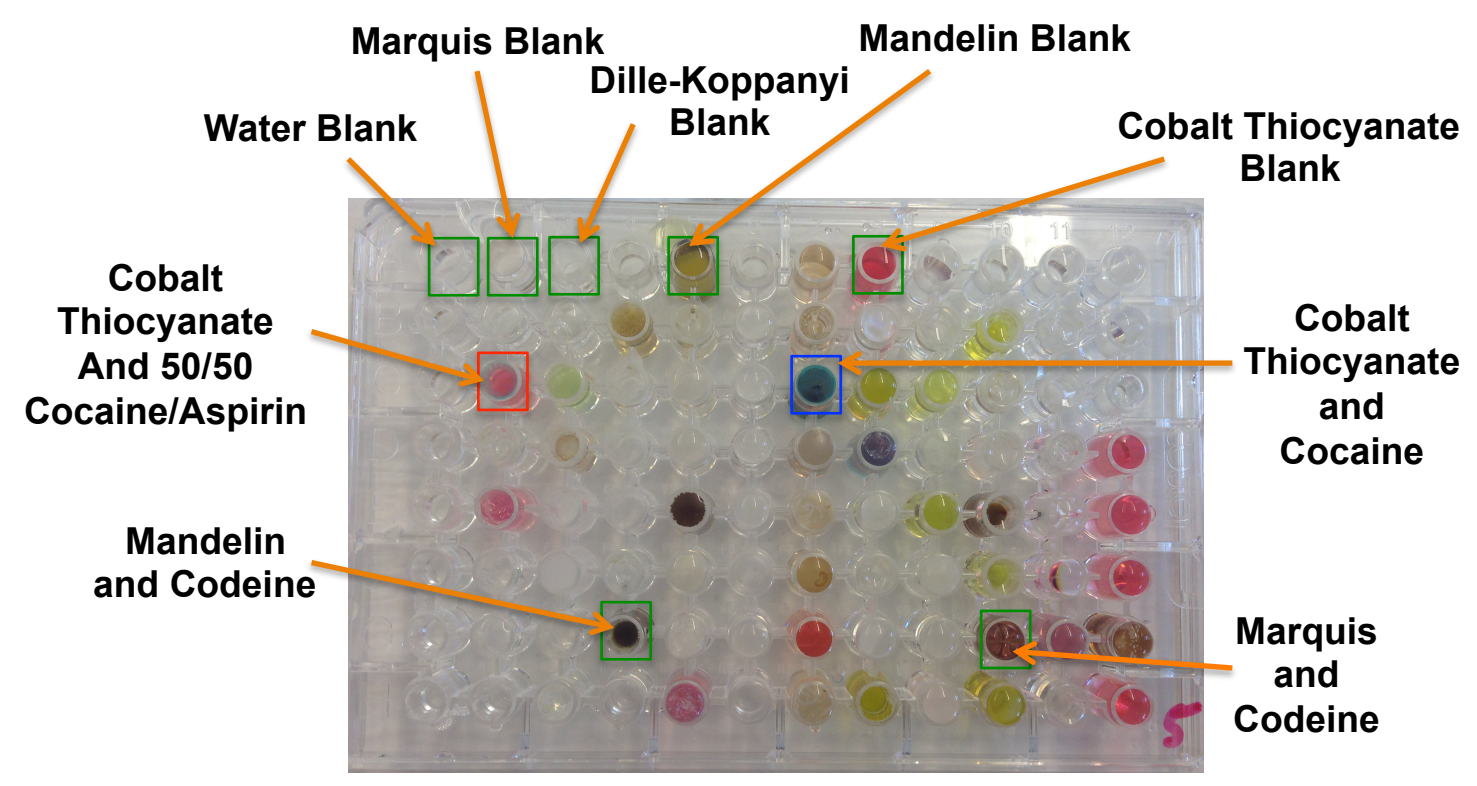

Figure 4.1. Photograph of a 96-well plate taken with Protea's built in camera prior to analysis using LAESI-MS. The colored wells either contain drug and reagent or a blank of a color test reagent. The red square indicates the reaction of $2.5 \mathrm{mg} / \mathrm{mL}$ each of cocaine and aspirin with cobalt thiocyanate and the blue square indicates the reaction of a $5 \mathrm{mg} / \mathrm{mL}$ cocaine with cobalt thiocyanate. Green squares indicate other wells of interest within the well-plate. All the wells contain solution, but not all are expected to be colored.

The Dille-Koppanyi reagent gave the fewest positive results and was only able to identify the drug in $21 \%$ of the cases studied. The Dille-Koppanyi reagent is made by combining cobalt (II) acetate dehydrate, glacial acetic acid, methanol, and isopropylamine, so is devoid of water and is quite volatile. Whereas the methanol present is ordinarily a reliable matrix replacement for water, the relatively long overall analysis time for the 96-well plate results in significant evaporative losses and concomitant decrease in signal yield for this particular reagent. The latest commercial LAESI source is now equipped with a chiller to help prevent evaporation and 
maintain frozen tissue samples, but this was not available at the time of the experiments. The Dille-Koppanyi reagent therefore could prove more reliable when well plates are properly filled and chilled to prevent evaporation.

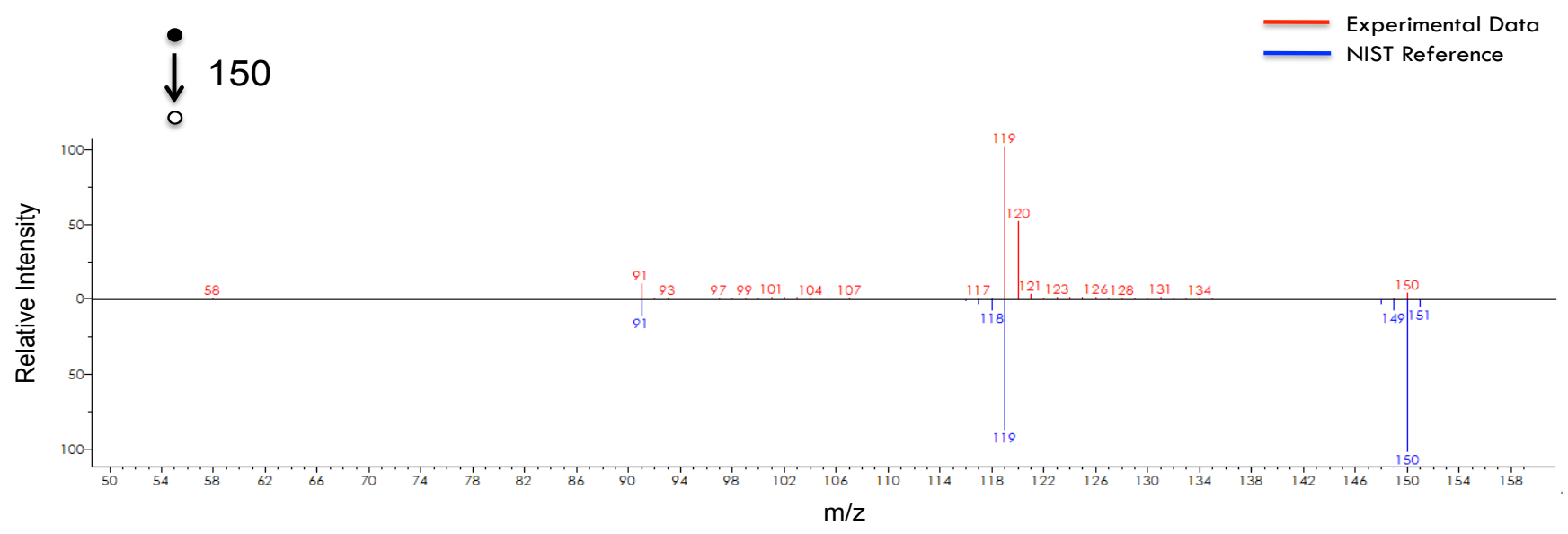

Figure 4.2. An example of the comparison between the experimental and NIST reference data for a positive identification of amethamphetamine standard. The experimental data (top in red) is shown head-to-tail with the NIST reference spectrum (bottom in blue).

Figure 4.3 shows that some drugs, like cocaine, are identified most of the time and other drugs, like phenobarbital, are rarely identified. The difference in observation frequency is attributed largely to the $\mathrm{pKa}$ of the drugs and their relative degree of protonation. Negative ion mode was not explored with these samples because of the general lack of negatively charged Brønsted-Lowry basic sites. Figure 4.4 shows a plot the pKa of each drug versus the total number of identifications of that drug in all reagents at all concentrations. The pKa values used in this plot were taken from the literature. ${ }^{219-225}$ The square of the linear correlation coefficient $\left(\mathrm{R}^{2}\right)$ of 0.69 means that the $\mathrm{pKa}$ accounts for $69 \%$ of the variance of the identifications. The main discrepancies in this plot are cocaine and quinine. 
Based on pKa alone, one would expect an equal degree of ionization and an equal number of identifications for cocaine and quinine. Although they both have very similar $\mathrm{pKa}$ values $($ cocaine $=8.6$ and quinine $=8.7)$, cocaine has a greater number of observations than expected and quinine has a fewer number of observations than expected. Quinine has a second, much weaker, Brønsted-Lowry basic site — with a conjugate pKa of 4.3 - so this site was

a)

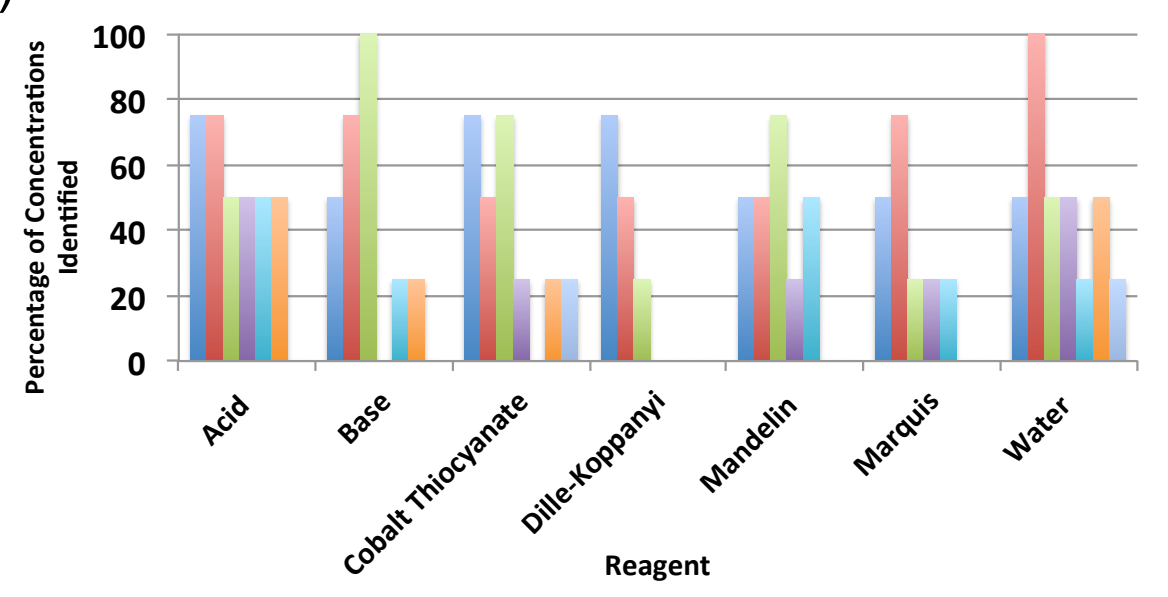

$$
\begin{aligned}
& \text { Cocaine } \\
& \text { Methamphetamine } \\
& \text { Amphetamine } \\
& \text { Oxycodone } \\
& \text { Codeine } \\
& \text { Quinine } \\
& \text { Phenobarbital }
\end{aligned}
$$

b)

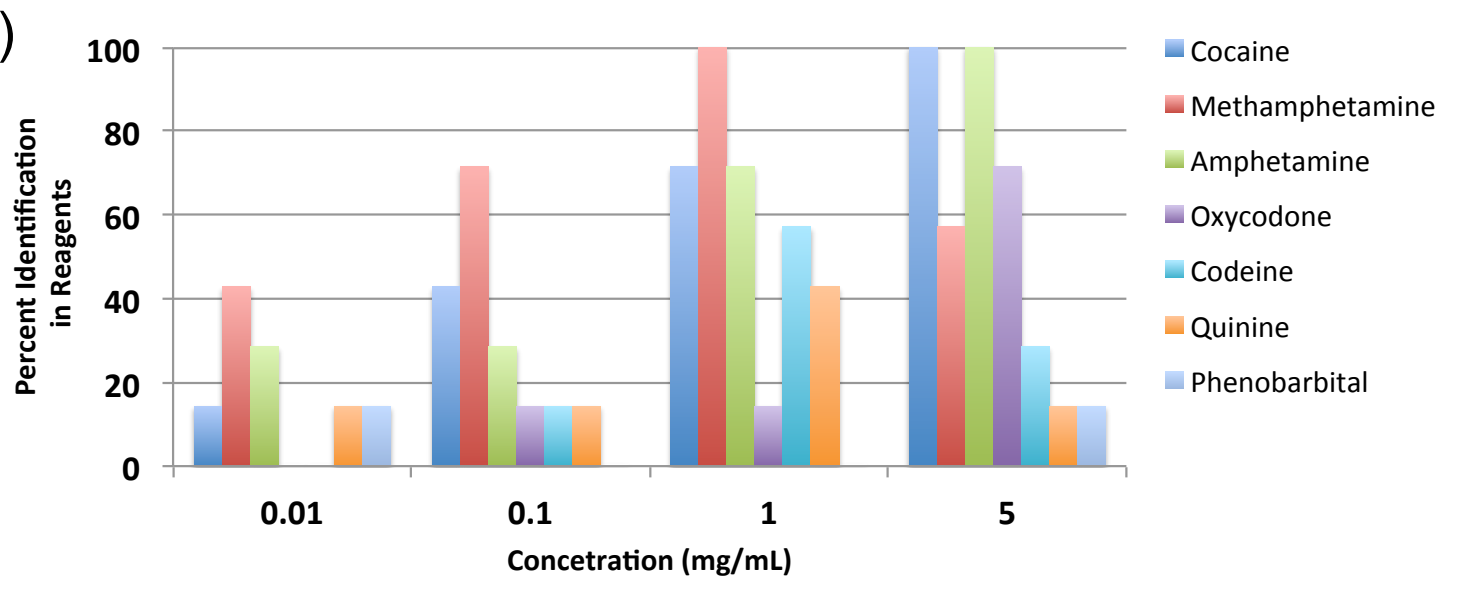

Figure 4.3. Summary of the positive identifications for each drug displaying the percentage of all the concentrations of a drug identified in each reagent and displaying the percent of all the reagents a drug is identified. 
not considered. In hindsight, we learned that the solubility of quinine is only around 0.10

$\mathrm{mg} / \mathrm{mL}$, whereas the solubility of cocaine is around $2500 \mathrm{mg} / \mathrm{mL}^{226,227}$ so it is likely that quinine actually exceeded its saturation limit, which prevented it from competing with cocaine for protons at many of the concentrations studied.

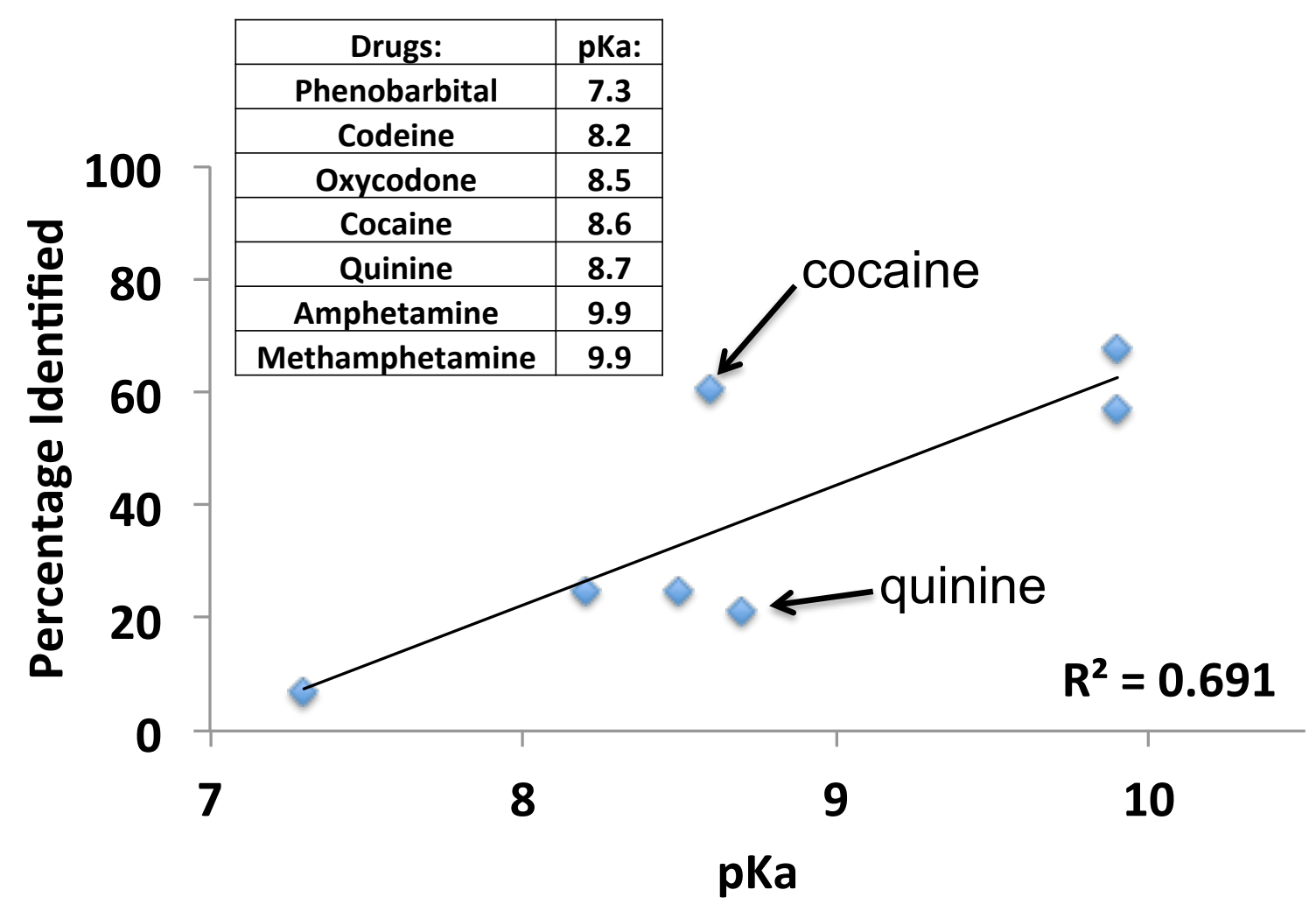

Figure 4.4. Plot of the number of positive drug identifications versus the $\mathrm{pKa}$ of the conjugate acid form of the individual drugs. Each drug and their corresponding $\mathrm{pKa}$ are identified in the inset table.

One important item to note is that the mechanism of the color change was not consistent between all of the color reagents that were utilized. For example, the Marquis reagent creates a covalently bonded complex to create the color change $\mathrm{e}^{233}$ while the cobalt thiocyanate reagent instead relies on the formation of an ionically bound cobalt complex. ${ }^{234}$ These different mechanisms of color formation could be another reason why we are seeing differences in the 
percent identifications between the drugs. The different complexes formed for the color reagents may amplify or inhibit the ability of LAESI to make these identifications. The data from this experiment however suggests that the mechanism of color formation does not play a large part in inhibiting the instruments ability to identify a drug. When a color reagent is added to a solution with a compatible drug, the drug and the color reagent will form a complex that creates the color. During our experiment LAESI is using the non-reacted drug in order to make its identification. As part of this experiment we did look for, but were unable to identify any drug-reagent complexes in our samples In Figure 4.3 we see the comparison of the percent identification of drugs in different reagents. When we compare the identifications in the cobalt thiocyanate and Marquis reagents we see that LAESI is able to identify drugs that are both reactive and nonreactive to both reagents. For example, codeine is not reactive with cobalt thiocyanate and cocaine is not reactive with Marquis reagent but they are both able to be identified by LAESI. By this logic then, the less drug that reacts with the color reagent the more drug that should be available for identification. So, something like quinine or phenobarbital that are not compatible with the Marquis reagent should be identified more easily because there is more drug available for the identification. On top of this if the color reagent or its products were inhibitive to identification then we would expect to see a lower percent identification for drugs that do react with color reagents both because these new complexes could obscure the identifications and because the formation of these complexes would lower the overall concentration of drug in solution to be used in identification. Contrary to this idea we are able to identify drugs in solutions in which they react like cocaine in cobalt thiocyanate. When the drugs are analyzed in a solution containing just water or a solution containing water and a little bit of acid LAESI is able to identify almost every drug analyzed in this experiment. This observation would suggest that 
the identification of the drugs may rely more on their ability to maintain high protonated populations in solution rather then their reaction to a color reagent. Those solutions Experimental Data

a)

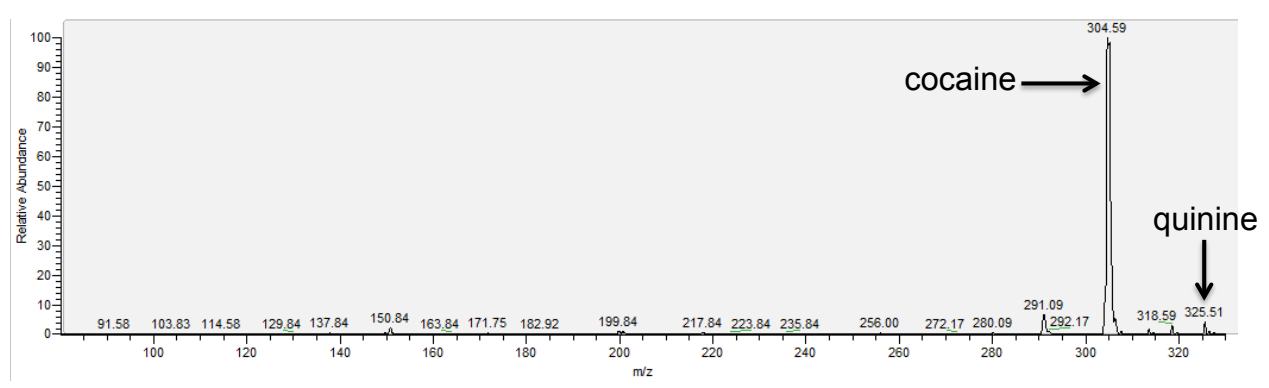

b)

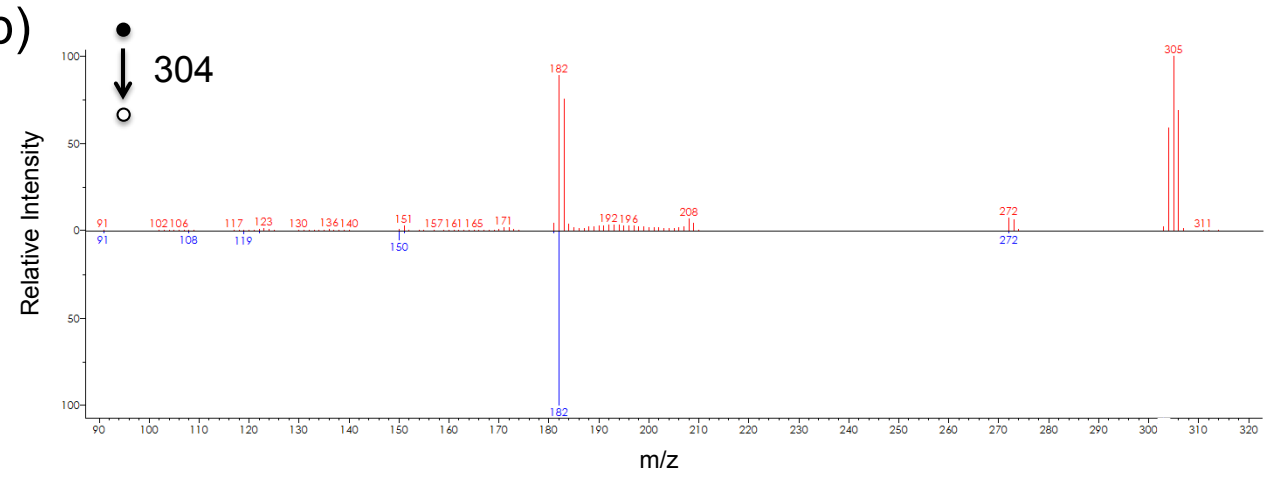

c)

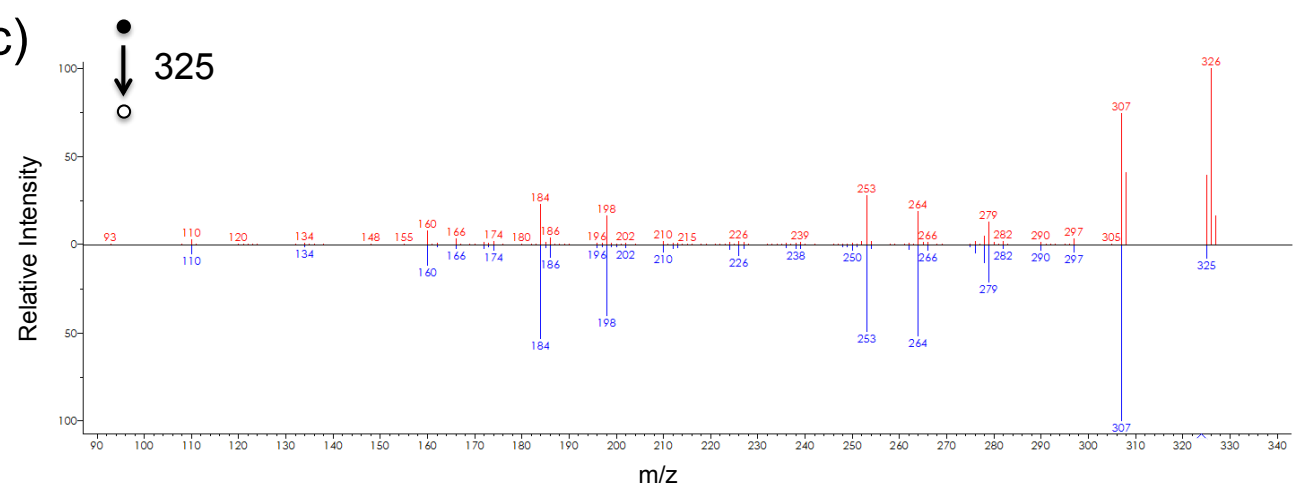

Figure 4.5. Comparison of a) full-scan MS spectra of 50:50 quinine $(\mathrm{m} / \mathrm{z} 325)$ and cocaine $(\mathrm{m} / \mathrm{z}$ 304 ) as well as b) the tandem MS of the $\mathrm{m} / \mathrm{z} 304$ cocaine precursor ion and c) the tandem MS spectrum of the $\mathrm{m} / \mathrm{z} 325$ quinine precursor ion. 
that are more amenable to increasing the amount of protons in solution, like an acid, seem to increase the likelihood that a drug will be identified. Thus, the inability of LAESI to identify a drug like codeine in the cobalt thiocyanate solution may be attributed more to the lack of a strong proton donor then to the presence of the color reagent. Further experimentation would need to focus on the combination of the different color reagents and auxiliary chemicals, like a solution that is slightly acidified and has a color reagent added to it.

Two separate mixtures of drugs were also tested alongside the pure drugs, which included a mixture of cocaine and quinine and a mixture of cocaine and aspirin. When these mixtures were tested, both components could be identified in the cocaine and quinine mixture at $1 \mathrm{mg} / \mathrm{mL}$ and $5 \mathrm{mg} / \mathrm{mL}$ (although, as discussed earlier, the quinine effectively saturates at approximately $0.1 \mathrm{mg} / \mathrm{mL}$ ). Figure 4.5 shows an example of the tandem MS spectra for the identification of the components of the cocaine and quinine mixture in cobalt thiocyanate reagent, commonly used as a presumptive test for cocaine. In the case of cocaine and aspirin mixture, only the cocaine portion of the mixture could be identified successfully. This result is not surprising because the primary active ingredient in aspirin, acetylsalicylic acid, is not particularly soluble in water and does not carry a formal positive charge even in acidic conditions. The confluence of these two characteristics of aspirin results in only the positive identification of cocaine in the LAESIMS/MS experiment from a mixture of the two drugs. Negative ion mode was not explored for aspirin, but would be expected to be more applicable because of the carboxylic acid site.

Out of 252 possible positive results, LAESI-MS/MS identified drugs in 83 cases, or with a 37\% success rate. Many of the negative results have been explained above. The false negatives include 36 wells (14\% of the analyses) containing the Dille-Koppanyi reagent, which in general provided very few positives. The false negatives also include: 1) reagents containing a strong 
base and therefore hindering detection in positive mode; 2) phenobarbital, which gave very poor response in general; and 3) analytes at concentrations at or below the detection limits. Under more favorable conditions, such as with analytes at or above $1 \mathrm{mg} / \mathrm{mL}$ in aqueous acid solutions,

a)

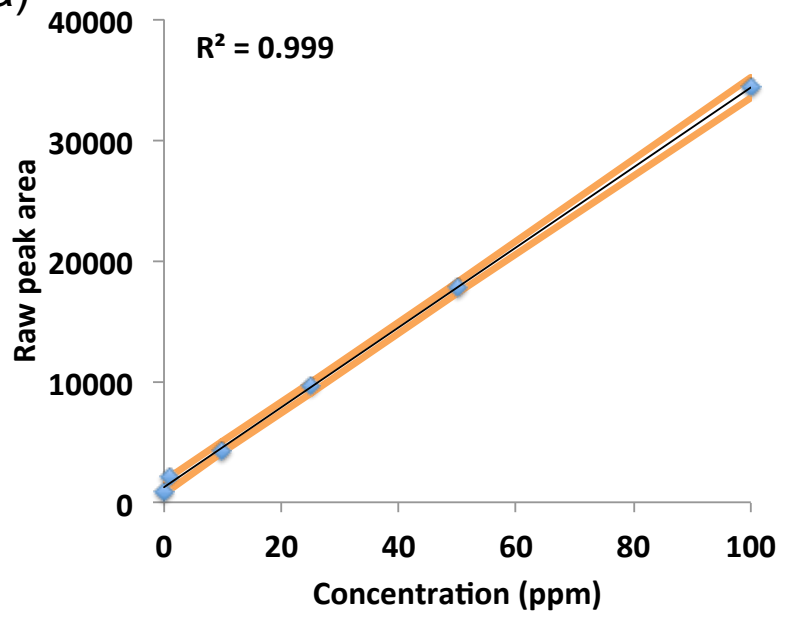

b)

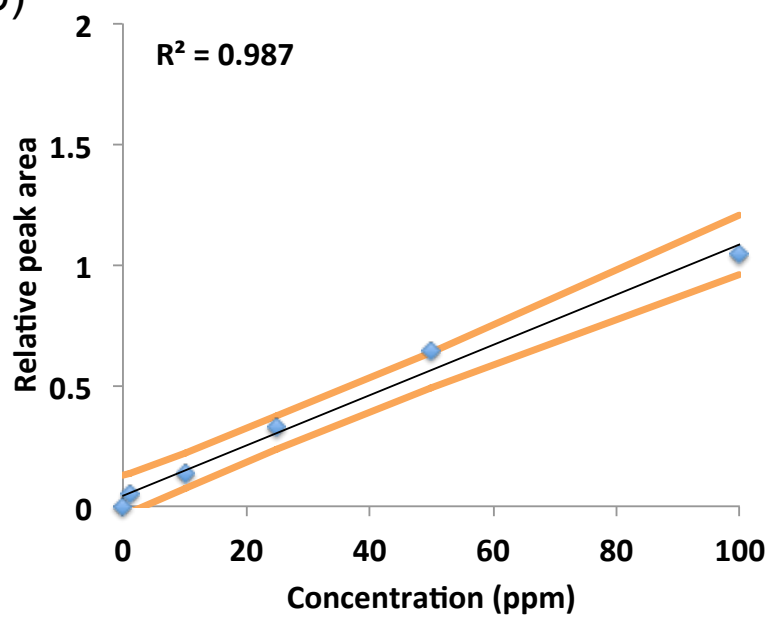

Figure 4.6. Calibration curves generated from the peak area of the tandem mass spectrum of a) raw methamphetamine signal and $b$ ) the ratio of raw methamphetamine peak area to the raw peak area of the deuterated methamphetamine. Each calibration curve is shown with the $95 \%$ confidence interval of the linear regression line.

the percentage of false negatives was less than $10 \%$, or greater than $90 \%$ true positives. We did not find any false positives, so carryover or cross-contamination was successfully avoided. Some early experiments with a concentrated solution of flunitrazepam resulted in carryover for tens of seconds, but such problems are generally avoidable under more controlled conditions.

To explore the ability to quantify analytes in an aqueous medium using LAESI, a calibration curve for methamphetamine was constructed ranging in concentration from 1-100 ppm. Deuterated $\left(\mathrm{D}_{14}\right)$ methamphetamine was also added as an internal standard at a constant concentration of $50 \mathrm{ppm}$ to each solution. The tandem mass spectra corresponding to non- 
deuterated and deuterated methamphetamine were integrated and the integrated peak areas plotted as a function of concentration, as shown in Figure 4.6. These plots show that the linear range for methamphetamine analysis using LAESI-MS extends from at least 1 to $100 \mathrm{ppm}$ with $\mathrm{R}^{2}$ values ranging from 0.999 for the absolute peak areas and 0.987 for the normalized peak areas. Although internal standards usually enhance quantitation with DESI ion sources relative to conventional external calibration, ${ }^{93}$ the use of an internal standard here could not have been expected to improve the excellent correlation in this case. In fact, quantitation using the deuterated internal standard was slightly worse. We anticipate that a more thorough validation study with different analytes would show that the use of deuterated internal standards would not be inferior to quantitation using absolute signals, but would either match or improve the correlation scores.

\subsubsection{Analysis of drugs in human hair sample}

Strands of drug-laced hair were studied using LAESI to determine if this system could identify drugs of abuse directly from the hair matrix, with minimal sample preparation. Ion mapping was also used to identify areas within the hair samples that may contain high abundances of a particular drug.

This series of experiments was performed on two different NIST hair strand reference samples (NIST SRM 8848 and SRM 2380) that contained several drugs ranging in concentration from $0.99 \mathrm{ng} / \mathrm{mg}$ to $11.9 \mathrm{ng} / \mathrm{mg}$. NIST SRM 8448 contained cocaine, benzoylecgonine, morphine and codeine and NIST SRM 2380 contained codeine, morphine, 6-monoacetylmorphine and tetrahydrocannabinol. Several strands of hair from either sample were attached to a microscope slide using double-sided Scotch tape, wetted with water and then analyzed using LAESI-MS without any further sample preparation. LAESI-MS was able to identify the presence of 
morphine, codeine, and cocaine in the hair samples but not the other drugs. Figure 4.7 shows MS/MS data for codeine and morphine two of the drugs analyzed using LAESI-MS/MS.

Comparison of both morphine and codeine with their associated NIST standards shows correspondence at most product ions. The use of an internal database of known drug standards would obviously improve spectral comparisons because of the known variance in product ion spectra with different instruments and conditions. ${ }^{228-230}$ Tetrahydrocannabinol, benzoylecgonine, and 6-monoacetylmorphine were unable to be identified directly from hair using LAESI-MS, presumably because they appeared at lower concentrations than the drugs that were observed.
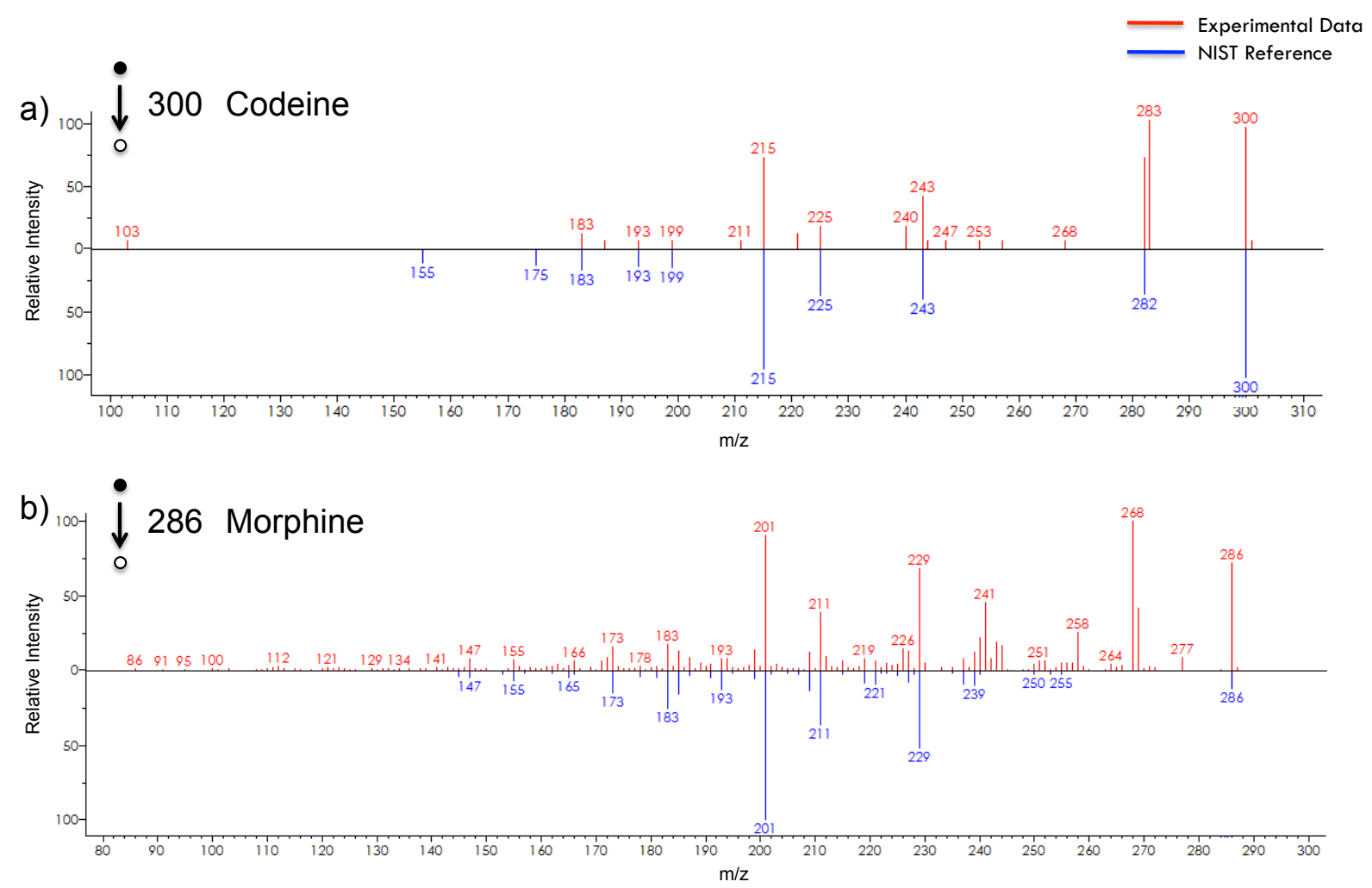

Figure 4.7. Head-to-tail comparison of the tandem MS spectra obtained directly from human hair a) of codeine at $\sim 7 \mathrm{ng} / \mathrm{mg}$ (precursor $\mathrm{m} / \mathrm{z} 300$ ) and b) morphine at $\sim 12 \mathrm{ng} / \mathrm{mg}$ hair (precursor $\mathrm{m} / \mathrm{z}$ 286). In each spectrum, the experimental data is shown in red while the NIST reference is shown in blue. 
All three unidentified drugs were present in the hair at concentrations lower than $2.10 \mathrm{ng} / \mathrm{mg}$ whereas all the identified drugs were present at concentrations above $6.7 \mathrm{ng} / \mathrm{mg}$. Further work will be necessary to lower the detection levels and widen the coverage, but the ability to identify at least three drugs of abuse at biologically relevant levels, without any extraction, separation or concentration, is a potential benefit over conventional methods of hair analysis.

To image the hair strands, the software was programmed to raster across the hair sampling every $200 \mu \mathrm{M}$ to $500 \mu \mathrm{M}$, depending on the size of the sample. The path of the laser could be directed to follow a single hair strand or to raster across an associated bunch of hair strands. MS/MS data could then be used to determine the distribution of morphine using the selected reaction monitoring transition of $\mathrm{m} / \mathrm{z} 286$ to $\mathrm{m} / \mathrm{z} 201$.

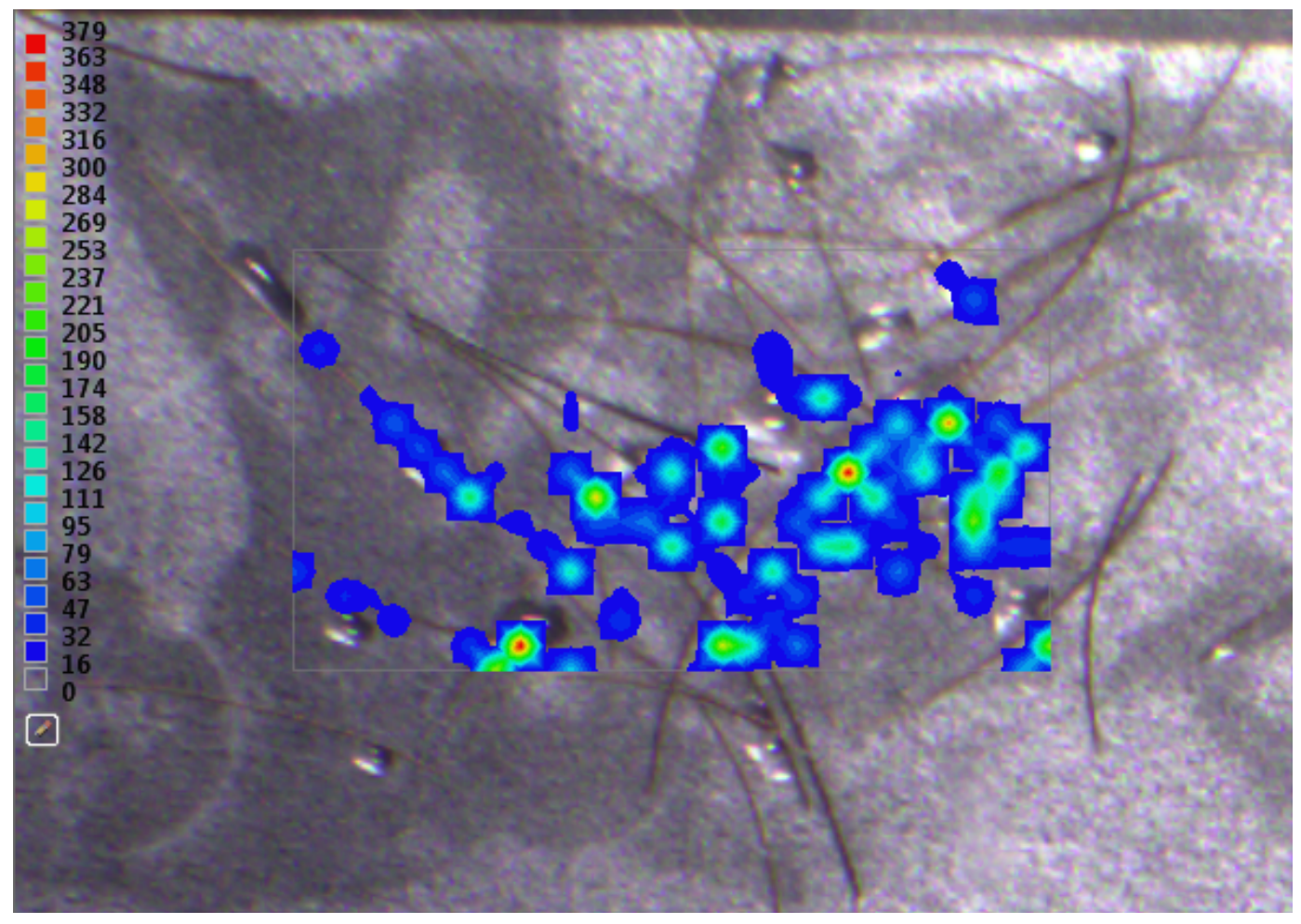

Figure 4.8. Overlay of the ion map of morphine onto the photographic image of the associated hair sample. The color scale associated with each of the ion maps indicates the absolute intensity of the product for the transition from $m / z 286 \rightarrow m / z 201$. 
An ion map generated for the abundance of morphine in the hair is shown in Figure 4.8. The color scale shows most abundant areas in red and the least abundant signals in blue. Figure 4.8 shows that morphine tends to appear in several points on a hair rather than appearing continuously across the entire strand. In the past, the analysis of hair samples has mainly been performed using GC-MS and currently an analysis of a single hair for cocaine and 6monoacetylmorphine can performed in 10 minutes using fast GC. ${ }^{235}$ However, ion maps may be advantageous because they present the distribution of the sample ions visually which may allow a more intuitive correlation between drug abundance and drug use over time. Other research groups have recognized this potential for ion maps and have pursued the technique. For example, Miki et. al. were able to effectively map the distribution of methamphetamine in hair using MALDI-TOF and MALDI-FT-ICR. ${ }^{236}$ This study utilized hair samples taken from individuals who had used drugs and thus they were investigating the abundance of drugs that had been incorporated into the hair strand. They were able to identify and map both drugs after the hair strand had been vertically cleaved so that the MALDI system could more easily reach the inside of the hair strand. This differs from our LAESI experiments in that it was not necessary for us to do anything to the hair in order to obtain a result. One reason for this may be that our hair samples were NIST reference strands that had the drug administered to the hair by soaking it in a drug solution for an extended period of time. In this way it there may have been more drug present on the outside of the hair strand in our reference samples then would be in a biological hair sample making it the extra sample preparation step unnecessary during our LAESI analysis. At this time we are still investigating whether the areas of high abundance that we observe in our ion maps are representative of the morphine distributed in the hair or if it is the product of the procedure itself. The regions of largest abundance tend to correlate with intersecting hairs, 
perhaps indicating that a larger surface area of hair is available for ablation in these regions. This is analogous to a laser ablation inductively coupled plasma mass spectrometry (LA-ICP-MS) experiment where it is suggested that high laser pulse energies or rastering should be used in order to obtain a representative sampling of the analyte. ${ }^{237}$ In this way then an area that has more surface area due to having overlapping hair strands has the possibility to produce more sample analyte when exposed to the laser pulse in either situation, assuming that both hair strands contain the same amount of drug. Despite this effect, the ion map for morphine still correlates well with the individual hair shafts.

\subsubsection{Direct analysis of drugs in plant matter}

Cannabis leaves were also studied using LAESI-MS to determine if this system could identify THC directly from the plant matrix without prior sample preparation. Ion mapping was also used to better understand how the psychotropic chemical THC is distributed throughout the leaf.

In one case, a leaf was fixed onto a glass depression slide and the leaf was wetted with water prior to analysis. After wetting, the leaves were analyzed directly by LAESI-MS. The MS/MS results are shown in Figure 4.9 wherein the data is compared to a reference spectrum for THC taken using another ambient technique, desorption atmospheric pressure photoionization (DAPPI). ${ }^{231}$ In addition to DAPPI, other ambient techniques like DESI have also been used to analyze cannabis leaves. ${ }^{19,232}$ The two MS/MS spectra in the supplemental material show similar fragment ions at $\mathrm{m} / \mathrm{z} 259, \mathrm{~m} / \mathrm{z} 233$ and $\mathrm{m} / \mathrm{z} 193$ and $\mathrm{m} / \mathrm{z}$ 135, which are characteristic of both THC and the non-psychoactive cannabinoid, cannabidiol (CBD), ${ }^{19}$ which is also listed as a schedule I controlled substance in the USA. We have not yet attempted to distinguish between 
the two cannabinoids based on tandem mass spectra alone, although the NIST spectra would indicate that such discrimination ought to be possible to a reasonable degree of confidence.

During the analysis of the wetted leaves without fixing, the ablation process caused the leaf matter to move around in the well during analysis, which is undesirable. To prevent movement, the plant matter was subsequently secured with the commonly used mounting media Permount solution or paraffin wax. Figure 4.10 shows the full-scan MS analysis of the paraffin and permount matrix compared to the normal LAESI background and shows no noticeable additional matrix ions from either media relative to the normal LAESI background.

a)

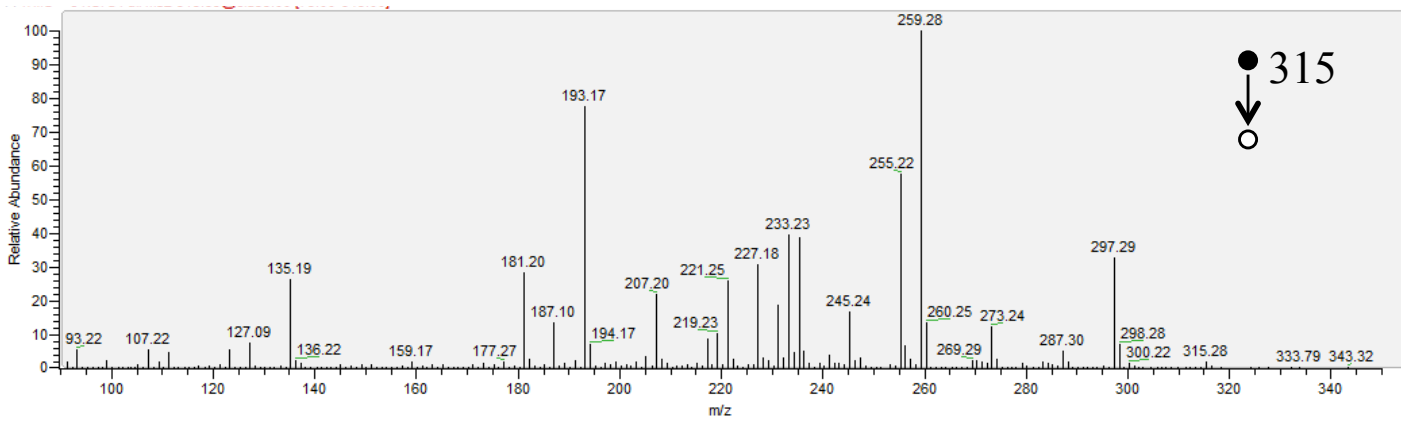

b)

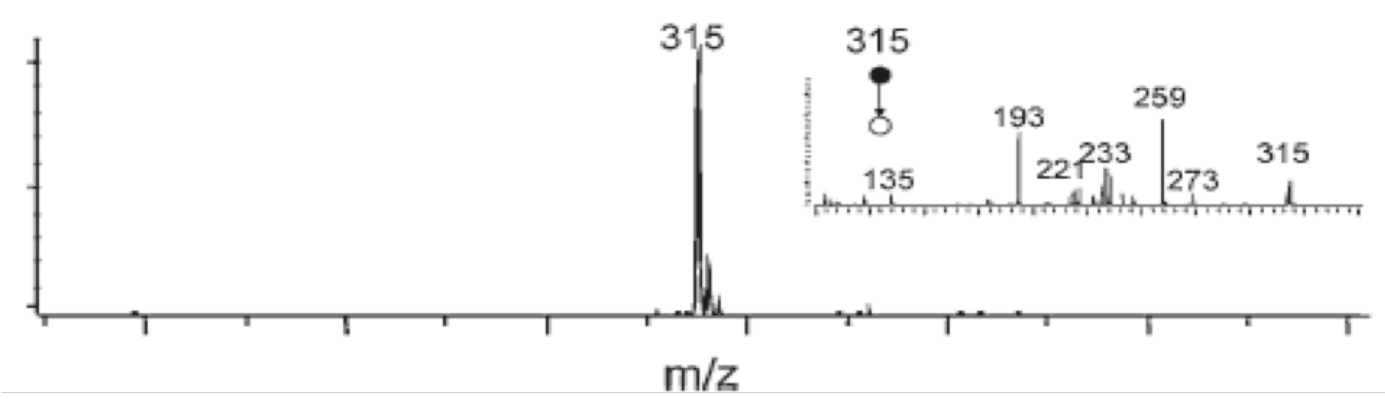

Figure 4.9. MS/MS data for the analysis of THC in a cannabis leaf a) with LAESI and $b$ ) with DAPPI. (Reprinted with permission from Kauppila, Tiina J.; Arvola, Ville; Haapala, Markus; Pol, Jarolslav; Aalberg, Laura; Saarela, Ville; Framssila, Sami; Kotiaho, Tapio; Kostiainen, Risto. Direct analysis of illicit drugs by desorption atmospheric pressure photoionization. Rapid Commun. Mass Spectrom. 2008, 22, 979-985.) 
To mount the cannabis leaf, paraffin wax was melted with a heat gun and allowed to drip onto a blank microscope slide. The leaf was then pressed into the liquid wax and held in place as the wax dried. Figure 4.11 in the supplemental section shows the MS/MS data obtained for the leaf mounted using paraffin wax. These spectra show that the mounting media has no deleterious effect on the MS/MS fragmentation pattern of THC. To image the leaf, the software was programmed to raster the leaf in a grid-like array every $500 \mu \mathrm{m}$ in a $1 \mathrm{~cm} \mathrm{x} 4 \mathrm{~cm}$ array. MS/MS

a) MS of paraffin
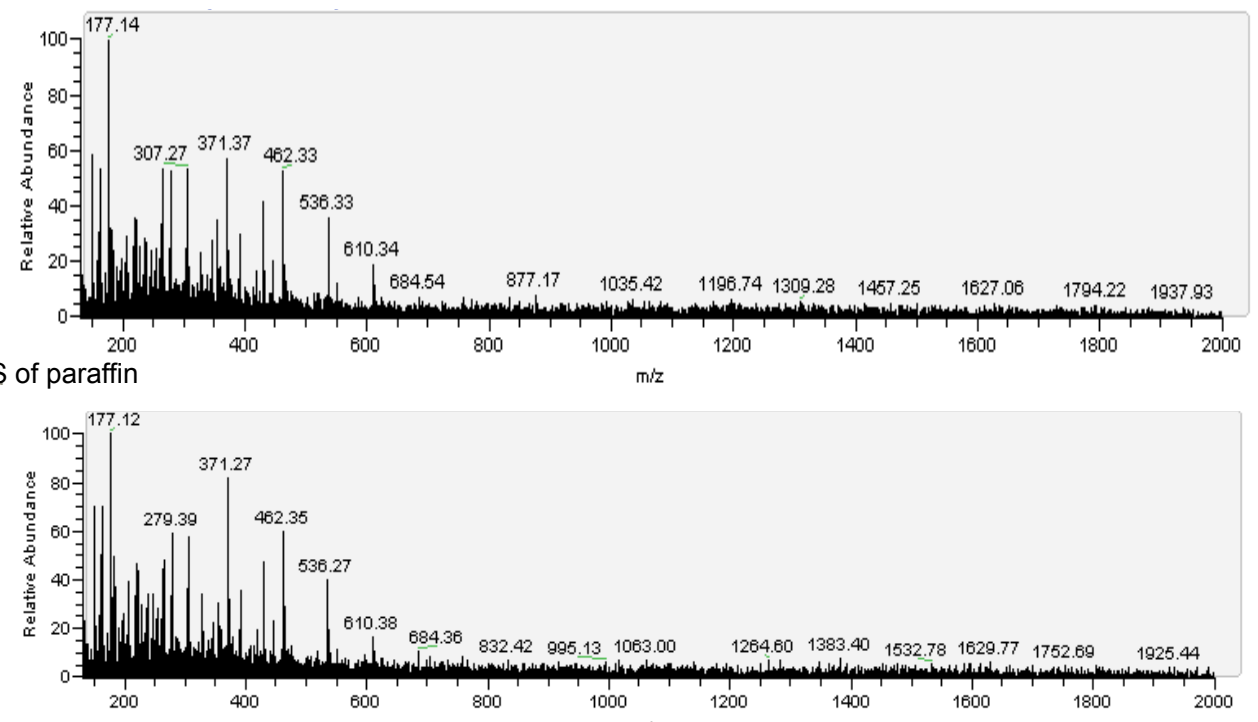

b) MS of permount solution

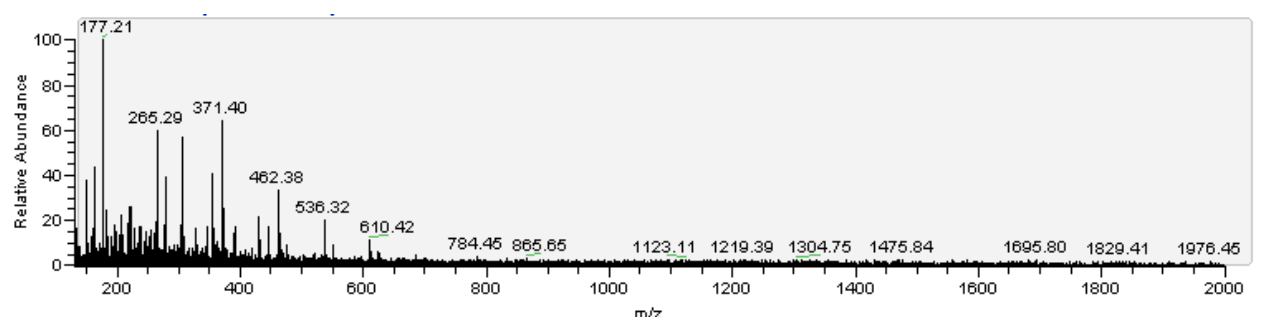

c) Normal LAESI Background

Figure 4.10. The comparison of a) the MS data for paraffin wax and b) the MS data for permount solution with c) the typical background obtained during a LAESI analysis.

data could then be used to plot a selected-reaction-monitoring (SRM) image for the transition of $m / z 315 \rightarrow m / z 259$ for THC (and CBD) in the form of a relief map. The ion maps generated for a leaf mounted in Permount and paraffin wax solution are shown in Figure 4.12. The color scale 
shows most abundant areas in red and the least abundant signals in blue. For the leaf held with paraffin wax, the areas of high abundance tend to correlate with the leaf's veins. In this particular leaf, it appears that the majority of the observed THC is contained in the top half of the leaf. A second leaf mounted with permount solution showed lower levels of THC (and CBD), but the regions of abundant signal also correlate with the veins of the leaf. At this point, we have not

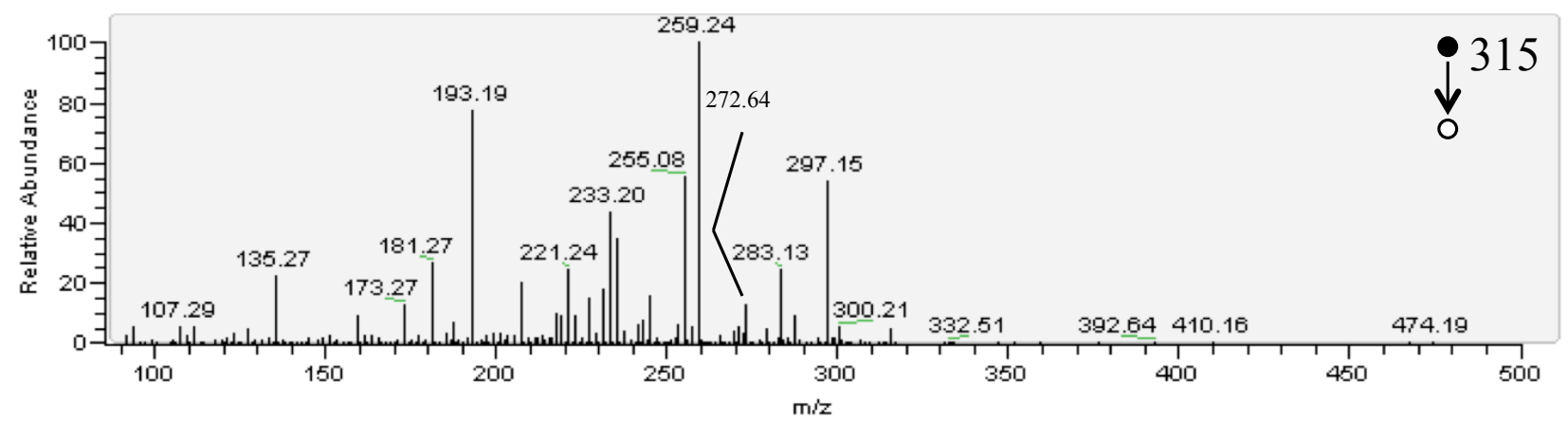

Figure 4.11. MS/MS data for the analysis of THC in a cannabis leaf mounted using paraffin wax with LAESI.

determined if THC content is actually greater at these points, or if the wetting and laser ablation efficiency is better at these points. From a forensic perspective, the distribution of THC in a leaf is less important than the ability to identify the psychoactive component itself, but follow-up studies could provide interesting biological information from the spatial distribution of compounds in the plant. Regardless of the reason, these preliminary experiments show that the veins of the leaf seem to provide the largest signal responses compared to other locations in the leaf.

\subsection{Conclusion}

The national backlog of drug samples in crime laboratories is typically analyzed using a variety of methods that almost always includes GC/MS. Current GC/MS methods are typically 
20 to 30 minutes long and - with the sample preparation and possible derivatization times included — severely limits the number of samples that can be analyzed per day. One way to relieve this backlog is to implement new instrumentation, which can quickly determine the

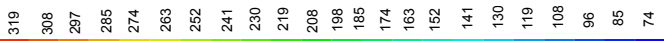

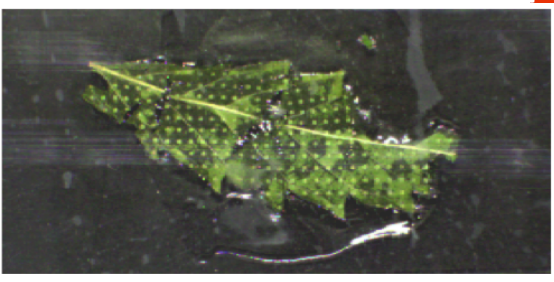

a) Marijuana leaf held with permount solution after LAESI analysis

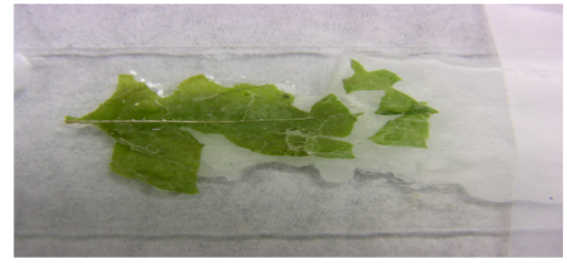

b) Marijuana leaf held with paraffin wax before LAESI analysis

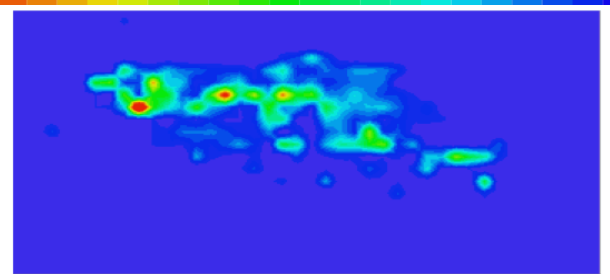

c) Ion map of permount mounted leaf
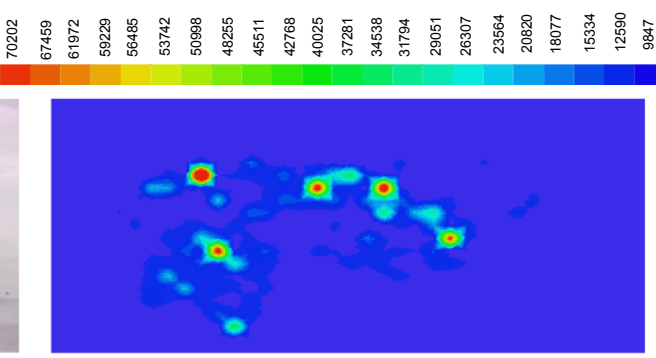

d) Ion map of paraffin mounted leaf

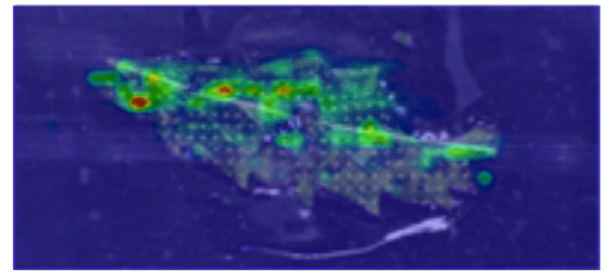

e) Overlay of ion map on permount mounted leaf

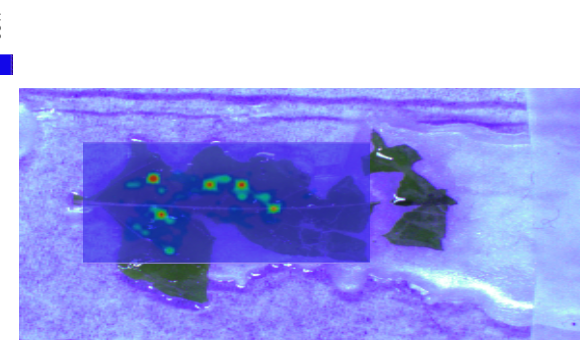

f) Overlay of ion map on paraffin mounted leaf

Figure 4.12. A cannabis leaf held onto a microscope slide with a) permount solution after LAESI analysis and b) paraffin wax before LAESI analysis, their associated ion maps c) for the permount mounted leaf $d$ ) for the paraffin mounted leaf and the overlay of the leaf image and its associated ion map for e) the permount mounted leaf and f) the paraffin mounted leaf. The color scale associated with each of the ion maps indicates the absolute intensity of the product for the transition from $m / z 315 \rightarrow m / z 259$, which is selective for THC \& CBD.

identity of drugs on a presumptive test, without the need to prepare a new sample. Herein, we provide preliminary results to show that LAESI-MS/MS can analyze 96 solution-based color-test samples in 30 minutes at an average time of $\sim 20$ seconds each. LAESI-MS/MS is able to quickly and easily identify the presence of a drug in an unknown sample with approximately $60 \%$ of the successful identifications occurring at a concentration at or above $1 \mathrm{mg} / \mathrm{mL}$. The ability to successfully detect a variety of drugs in different reagents and to construct the calibration curves 
for methamphetamine illustrates two different ideas about LAESI-MS. The first is that LAESIMS can detect drugs of abuse at concentrations between 1 and 5000 ppm. Secondly, LAESIMS/MS analyses can produce a linear range for methamphetamine from at least 1 to $100 \mathrm{ppm}$. LAESI-MS was also shown to be able to identify mixtures of drugs in solution and should be capable of identifying minor impurities, cutting agents or adulterants. Chemical spot tests are typically used in the range of $2 \mathrm{mg} / \mathrm{mL}$ and $4 \mathrm{mg} / \mathrm{mL}^{205}$ or $2000 \mathrm{ppm}$ and $4000 \mathrm{ppm}$ which can easily and reliably be detected in the LAESI-MS/MS range tested. In addition, LAESI-MS can detect drugs at concentrations lower than the concentration required for a positive spot test. Therefore LAESI-MS can be used to minimize false negatives from color spot tests.

LAESI-MS can also identify drugs in hair and plant samples although significant work lies ahead to validate and quantify drugs in these more difficult applications. Still, the ability to screen hair and botanical matter for drugs of abuse in a matter of seconds may complement existing techniques in forensic and first responder applications. 


\section{CHAPTER 5 : FUTURE WORK AND CONCLUSIONS}

Lipid studies in the past have traditionally been achieved through MS/MS analysis using both low and high energy CID. Extensive fragmentation of lipid molecules has been demonstrated using HE-CID, while low-energy CID typically produces less abundant fragmentation primarily generating fragments of the headgroup and the cleavage of the entire fatty acid chain. Because instruments that can perform HE-CID are much less common, many labs only have the option to study these lipid molecules using low-energy CID. In this work it has been demonstrated that MAD of lipid molecules can generate the products of HE-CID like experiment in a low-energy CID environment making the high-energy products available to more common instrument configurations.

Forensic identification of drugs of abuse has traditionally relied on the use of techniques like GC/MS for an accurate confirmation of presumptive test results. Although accurate and reliable these techniques are also saddled with long analysis times and lengthy sample preparation steps, which increase turn around time and cause backlogs to appear in forensic labs. To counteract this problem new techniques like LAESI are being developed. In this work, LAESI has been use to study several drugs of abuse in a variety of media including solutions, hair and plant matter. Drugs could be identified in solution at concentration both $1 \mathrm{mg} / \mathrm{mL}$ and 5 $\mathrm{mg} / \mathrm{mL}$ except phenobarbital which could only be identified at $1 \mathrm{mg} / \mathrm{mL}$. These drugs could be identified in solutions in which a presumptive color test had already been performed. Drugs identified in the hair and plant matter could be detected at forensically relevant levels. Data acquired from each analysis could also be utilized to generate ion maps that showed the density of a particular drug across a sample. LAESI was able to identify drugs of abuse in each media much more rapidly and with less extensive sample preparation then that associated with GC/MS. 
Overall, LAESI showed promise for the analysis of drugs and should function efficiently and effectively for this application.

The experiments performed demonstrate the characterization of glycerophospholipids using MAD. Glycerophospholipids are only a small portion of the types of lipids present in a biological system. In the future the scope of the lipid analysis could be expanded to further explore other lipid families like sterols, glycerolipids or sphingolipids. The lack of a phosphocholine group in the glycerolipids and sterols may lead to differences in radical fragmentation patterns as the metastable atom is forced to ionize the lipid molecules at a different starting point then phosphocholine oxygen typically associated glycerophospholipids. Studying lipids that do not contain a phosphocholine headgroup like triacylglycerol (a glycerolipid) will also allow us to observe how MAD fragments lipids that more easily retain multiple charges and whether presence of these extra charges has any influence on the type of fragmentation that occurs.

The experiments performed on singly protonated lipids has shown that there is potential for the identification of double bond location in glycerophospholipids through the identification of fragmentation groups containing large abundances McLafferty rearrangement products. The potential to identify these double bonds could be examined further by examining a series of less complex systems like a series of mono-, di-, or tri-acylglycerols with gradually increasing unsaturation. By moving in series from a monoacylglycerol to a diacylglycerol to a glycerophospholipid one could observe which fragments or fragmentation patterns are indicative of double bonds within the fatty acid chain using MAD. The identification and explanation of these key fragments would be easier in these simpler systems especially when MAD fragmentation has been shown to exhibit competition between radical and even electron 
fragmentation pathways often resulting in spectra that are filled with multiple groups of even and odd electron products.

In the forensic experiments it was shown that LAESI could identify drugs of abuse in a variety of media including hair and plant samples. The data from the hair and plant samples were then used to generate 2-D ion maps that showed the distribution of a particular drug across the media. One interesting area of future research would be the attempt to correlate the signal obtained in a 2-D ion map with a particular characteristic of the media itself. This study could contribute useful information about each media helping to answer questions about how the content of THC changes in a marijuana leaf as it dries or where a particular drug is distributed in a person's hair strand over time. The association of the 2-D ion mapping technology with the determination or identification of a drug characteristic would shift this ability from a novelty to a practical technique making LAESI more appealing for forensic analysis.

Aside from ion mapping future directions for the project could also focus on optimizing experimental parameters necessary for a successful identification. These parameters range from the optimal solvent to use for hydrating the sample to the length of time a sample tray can be stored before the solvent volume is to low for a successfully analysis. These kinds of parameters are important to quantify in order to create methods that can be automated and easily implemented in forensic laboratories especially when is competing with techniques like GC/MS that have been rigorously tested through many years of laboratory use within the scientific community. Once the optimal parameters for LAESI are established the types of drugs and color reagents can be expanded from the original seven drugs and four color reagents that were analyzed in this original experiment. By expanding the number of drugs and reagents we can assure that there is not a particular drug, color reagent or drug and color reagent product that 
causes interferes with LAESI's ability to make a successful identification further validating its effectiveness in this application. 


\section{REFERENCES:}

1. Zubarev, R. A.; Kelleher, N. L.; McLafferty, F. W., Electron capture dissociation of multiply charged protein cations. A nonergodic process. J. Am. Chem. Soc. 1998, 120 (13), 3265-3266.

2. Zubarev, R. A.; Kruger, N. A.; Fridriksson, E. K.; Lewis, M. A.; Horn, D. M.; Carpenter, B. K.; McLafferty, F. W., Electron Capture Dissociation of Gaseous Multiply-Charged Proteins Is Favored at Disulfide Bonds and Other Sites of High Hydrogen Atom Affinity. J. Am. Chem. Soc. 1999, 121 (12), 2857-2862.

3. Zubarev, R. A.; Horn, D. M.; Fridriksson, E. K.; Kelleher, N. L.; Kruger, N. A.; Lewis, M. A.; Carpenter, B. K.; McLafferty, F. W., Electron capture dissociation for structural characterization of multiply charged protein cations. Anal. Chem. 2000, 72 (3), 563-573.

4. Zubarev, R. A., Electron-capture dissociation tandem mass spectrometry. Curr. Opin. Biotechnol. 2004, 15 (1), 12-16.

5. Syka, J. E. P.; Coon, J. J.; Schroeder, M. J.; Shabanowitz, J.; Hunt, D. F., Peptide and protein sequence analysis by electron transfer dissociation mass spectrometry. $\mathrm{P}$ Natl Acad Sci USA 2004, 101 (26), 9528-9533.

6. Coon, J. J.; Shabanowitz, J.; Hunt, D. F.; Syka, J. E. P., Electron Transfer Dissociation of Peptide Anions. J. Am. Soc. Mass. Spectrom. 2005, 16 (6), 880-882.

7. Good, D. M.; Wirtala, M.; McAlister, G. C.; Coon, J. J., Performance Characteristics of Electron Transfer Dissociation Mass Spectrometry. Cell. Mol. Biol. 2007, 6 (11), 19421951.

8. Wiesner, J.; Premsler, T.; Sickmann, A., Application of electron transfer dissociation (ETD) for the analysis of posttranslational modifications. PROTEOMICS 2008, 8 (21), 4466-4483.

9. Cook, S. L.; Collin, O. L.; Jackson, G. P., Metastable atom-activated dissociation mass spectrometry: leucine/isoleucine differentiation and ring cleavage of proline residues. $J$. Mass Spectrom. 2009, 44 (8), 1211-1223.

10. Cook, S. L.; Zimmermann, C. M.; Singer, D.; Fedorova, M.; Hoffmann, R.; Jackson, G. P., Comparison of CID, ETD and metastable atom-activated dissociation (MAD) of doubly and triply charged phosphorylated tau peptides. J. Mass Spectrom. 2012, 47 (6), 786-794.

11. Cook, S. L.; Jackson, G., Metastable atom-activated dissociation mass spectrometry of phosphorylated and sulfonated peptides in negative ion mode. J. Am. Soc. Mass. Spectrom. 2011, 22 (6), 1088-1099.

12. Dodds, E. D.; McCoy, M. R.; Rea, L. D.; Kennish, J. M., Gas chromatographic quantification of fatty acid methyl esters: flame ionization detection vs. electron impact mass spectrometry. Lipids 2005, 40 (4), 419-428.

13. Thurnhofer, S.; Vetter, W., A gas chromatography/electron ionization-mass spectrometry-selected ion monitoring method for determining the fatty acid pattern in food after formation of fatty acid methyl esters. Journal of agricultural and food chemistry 2005, 53 (23), 8896-8903.

14. Barber, M.; Bordoli, R. S.; Sedgwick, R. D.; Tyler, A. N., Fast atom bombardment of solids (FAB): A new ion source for mass spectrometry. J. Chem. Soc., Chem. Commun. 1981, (7), 325-327.

15. Kinter, M.; Sherman, N. E., Protein Sequencing and Identification Using Tandem Mass Spectrometry. Wiley: 2005. 
16. Williams, P., Secondary ion mass spectrometry. Annu. Rev. Mater. Sci. 1985, 15 (1), $517-$ 548.

17. Sundqvist, B.; Macfarlane, R. D., 252Cf-Plasma desorption mass spectrometry. Mass Spectrom. Rev. 1985, 4 (4), 421-460.

18. Gross, J. H., Mass Spectrometry: A Textbook. 1st ed.; Springer: Heidelberg, Germany, 2004.

19. Lattimer, R. P.; Schulten, H.-R., Field ionization and field desorption mass spectrometry: past, present, and future. Anal. Chem. 1989, 61 (21), 1201A-1215A.

20. Barber, M.; Bordoli, R. S.; Elliott, G. J.; Sedgwick, R. D.; Tyler, A. N., Fast atom bombardment mass spectrometry. Anal. Chem. 1982, 54 (4), 645A-657A.

21. Roberts, W. L.; Santikarn, S.; Reinhold, V.; Rosenberry, T., Structural characterization of the glycoinositol phospholipid membrane anchor of human erythrocyte acetylcholinesterase by fast atom bombardment mass spectrometry. J. Biol. Chem. 1988, 263 (35), 18776-18784.

22. Barber, M.; Bordoli, R.; Sedgwick, R.; Tyler, A., Fast atom bombardment of solids as an ion source in mass spectrometry. Nature 1981, 293 (5830), 270-275.

23. Tian, R.; Facelli, J. C.; Michl, J., Vibrational and electronic spectra of matrix-isolated nitrogen trimer radical and azide. J. Phys. Chem. 1988, 92 (14), 4073-4079.

24. Schröder, E.; Münster, H.; Budzikiewicz, H., Ionization by fast atom bombardment-A chemical lonization (matrix) process in the gas phase. Org. Mass Spectrom. 1986, 21 (10), 707-715.

25. Münster, H.; Theobald, F.; Budzikiewicz, H.; Schröder, E., The formation of a matrix plasma in the gas phase under fast atom bombardment conditions. Int. J. Mass Spectrom. Ion Processes 1987, 79 (1), 73-79.

26. Rosen, R. T.; Hartman, T. G.; Rosen, J. D.; Ho, C.-T.; Budzikiewicz, H., Fast atom bombardment mass spectra of low molecular weight alcohols and other compounds. Evidence for a chemical ionization process in the gas phase. Rapid Commun. Mass Spectrom. 1988, 2 (1), 21-23.

27. Van Breemen, R. B.; Snow, M.; Cotter, R. J., Time-resolved laser desorption mass spectrometry. I. Desorption of preformed ions. Int. J. Mass Spectrom. Ion Phys. 1983, 49 (1), 35-50.

28. Musselman, B. D.; Watson, J. T.; Chang, C. K., Direct evidence for preformed ions of porphyrins in the solvent matrix for fast atom bombardment mass spectrometry. Org. Mass Spectrom. 1986, 21 (4), 215-219.

29. Shiea, J.; Sunner, J., The acid effect in fast atom bombardment. Org. Mass Spectrom. 1991, 26 (1), 38-44.

30. Tomer, K. B.; Jensen, N. J.; Gross, M. L., Fast atom bombardment and tandem mass spectrometry for determining structural modification of fatty acids. Anal. Chem. 1986, 58 (12), 2429-2433.

31. Deterding, L. J.; Gross, M. L., Fast-atom-bombardment and tandem mass spectrometry for determining structures of fatty acids as their picolinyl ester derivatives. Anal. Chim. Acta 1987, 200 (0), 431-445.

32. Cole, M. J.; Enke, C. G., Direct determination of phospholipid structures in microorganisms by fast atom bombardment triple quadrupole mass spectrometry. Anal. Chem. 1991, 63 (10), 1032-1038. 
33. Jensen, N.; Tomer, K.; Gross, M., Fast atom bombardment and tandem mass spectrometry of phosphatidylserine and phosphatidylcholine. Lipids 1986, 21 (9), 580588.

34. Ohashi, Y.; Iwamori, M.; Ogawa, T.; Nagai, Y., Analysis of long-chain bases in sphingolipids by positive ion fast atom bombardment or matrix-assisted secondary ion mass spectrometry. Biochemistry 1987, 26 (13), 3990-3995.

35. Tanaka, K.; Waki, H.; Ido, Y.; Akita, S.; Yoshida, Y.; Yoshida, T.; Matsuo, T., Protein and polymer analyses up to $\mathrm{m} / \mathrm{z} 100,000$ by laser ionization time-of-flight mass spectrometry. Rapid Commun. Mass Spectrom. 1988, 2 (8), 151-153.

36. Griffiths, J., A brief history of mass spectrometry. Anal. Chem. 2008, 80 (15), 5678-5683.

37. Karas, M.; Bachmann, D.; Hillenkamp, F., Influence of the wavelength in high-irradiance ultraviolet laser desorption mass spectrometry of organic molecules. Anal. Chem. 1985, 57 (14), 2935-2939.

38. Hillenkamp, F.; Karas, M.; Beavis, R. C.; Chait, B. T., Matrix-assisted laser desorption/ionization mass spectrometry of biopolymers. Anal. Chem. 1991, 63 (24), 1193A-1203A.

39. Kafka, A. P.; Kleffmann, T.; Rades, T.; McDowell, A., The application of MALDI TOF MS in biopharmaceutical research. Int. J. Pharm. 2011, 417 (1-2), 70-82.

40. Kirpekar, F.; Berkenkamp, S.; Hillenkamp, F., Detection of Double-Stranded DNA by IR- and UV-MALDI Mass Spectrometry. Anal. Chem. 1999, 71 (13), 2334-2339.

41. Pulfer, M.; Murphy, R. C., Electrospray mass spectrometry of phospholipids. Mass Spectrom. Rev. 2003, 22 (5), 332-364.

42. Shimma, S.; Kubo, A.; Satoh, T.; Toyoda, M., Detailed structural analysis of lipids directly on tissue specimens using a MALDI-SpiralTOF-ReflectronTOF mass spectrometer. PLoS One 2012, 7 (5), e37107.

43. enauer, E.; Allmaier, G., The renaissance of high-energy CID for structural elucidation of complex lipids: MALDI-TOF/RTOF-MS of alkali cationized triacylglycerols. J. Am. Soc. Mass. Spectrom. 2009, 20 (6), 1037-1047.

44. Pittenauer, E.; Allmaier, G., A universal product ion nomenclature for $[\mathrm{M}-\mathrm{H}]^{-},[\mathrm{M}+\mathrm{H}]^{+}$ and $[\mathrm{M}+\mathrm{nNa}-(\mathrm{n}-1) \mathrm{H}]^{+}(\mathrm{n}=1-3)$ glycerophospholipid precursor ions based on highenergy CID by MALDI-TOF/RTOF mass spectrometry. Int. J. Mass Spectrom. 2011, 301 (1-3), 90-101.

45. Marto, J. A.; White, F. M.; Seldomridge, S.; Marshall, A. G., Structural characterization of phospholipids by matrix-assisted laser desorption/ionization Fourier transform ion cyclotron resonance mass spectrometry. Anal. Chem. 1995, 67 (21), 3979-3984.

46. Gusev, A. I., Interfacing matrix-assisted laser desorption/ionization mass spectrometry with column and planar separations. Fresenius J. Anal. Chem. 2000, 366 (6-7), 691-700.

47. Guittard, J.; Hronowski, X. L.; Costello, C. E., Direct matrix-assisted laser desorption/ionization mass spectrometric analysis of glycosphingolipids on thin layer chromatographic plates and transfer membranes. Rapid Commun. Mass Spectrom. 1999, 13 (18), 1838-1849.

48. Preisler, J.; Hu, P.; Rejtar, T.; Moskovets, E.; Karger, B. L., Capillary array electrophoresis-MALDI mass spectrometry using a vacuum deposition interface. Anal. Chem. 2002, 74 (1), 17-25. 
49. Schiller, J.; Hammerschmidt, S.; Wirtz, H.; Arnhold, J.; Arnold, K., Lipid analysis of bronchoalveolar lavage fluid (BAL) by MALDI-TOF mass spectrometry and 31P NMR spectroscopy. Chem. Phys. Lipids 2001, 112 (1), 67-79.

50. Schiller, J.; Zschörnig, O.; Petkovic, M.; Müller, M.; Arnhold, J.; Arnold, K., Lipid analysis of human HDL and LDL by MALDI-TOF mass spectrometry and 31P-NMR. $J$. Lipid Res. 2001, 42 (9), 1501-1508.

51. Zabrouskov, V.; Al-Saad, K. A.; Siems, W. F.; Hill, H. H.; Knowles, N. R., Analysis of plant phosphatidylcholines by matrix-assisted laser desorption/ionization time-of-flight mass spectrometry. Rapid Commun. Mass Spectrom. 2001, 15 (12), 935-940.

52. Fenn, J. B., Ion formation from charged droplets: roles of geometry, energy, and time. $J$. Am. Soc. Mass. Spectrom. 1993, 4 (7), 524-535.

53. Fenn, J. B.; Mann, M.; Meng, C. K.; Wong, S. F.; Whitehouse, C. M., Electrospray ionization for mass spectrometry of large biomolecules. Science 1989, 246 (4926), 64-71.

54. Whitehouse, C. M.; Dreyer, R. N.; Yamashita, M.; Fenn, J. B., Electrospray interface for liquid chromatographs and mass spectrometers. Anal. Chem. 1985, 57 (3), 675-679.

55. Dole, M.; Hines, R. L.; Mack, L. L.; Mobley, R. C.; Ferguson, L. D.; Alice, M. B., Gas phase macroions. Macromolecules 1968, 1 (1), 96-97.

56. Dole, M.; Mack, L. L.; Hines, R. L.; Mobley, R. C.; Ferguson, L. D.; Alice, M. B., Molecular beams of macroions. J. Chem. Phys. 1968, 49 (5), 2240-2249.

57. Zeleny, J., Instability of electrified liquid surfaces. Phys. Rev. 1917, 10 (1), 1-6.

58. Taylor, G., Disintegration of water drops in an electric field. Proc. R. Soc. London, A 1964, 280 (1382), 383-397.

59. Gaskell, S. J., Electrospray: Principles and practice. J. Mass Spectrom. 1997, 32 (7), 677 688.

60. Kebarle, P., A brief overview of the present status of the mechanisms involved in electrospray mass spectrometry. J. Mass Spectrom. 2000, 35 (7), 804-817.

61. Kebarle, P.; Verkerk, U. H., Electrospray: From ions in solution to ions in the gas phase, what we know now. Mass Spectrom. Rev. 2009, 28 (6), 898-917.

62. Smith, R. D.; Loo, J. A.; Edmonds, C. G.; Barinaga, C. J.; Udseth, H. R., New developments in biochemical mass spectrometry: electrospray ionization. Anal. Chem. 1990, 62 (9), 882-899.

63. van Duijn, E.; Simmons, D. A.; van den Heuvel, R. H. H.; Bakkes, P. J.; van Heerikhuizen, H.; Heeren, R. M. A.; Robinson, C. V.; van der Vies, S. M.; Heck, A. J. R., Tandem Mass Spectrometry of Intact GroEL-Substrate Complexes Reveals SubstrateSpecific Conformational Changes in the trans Ring. J. Am. Chem. Soc. 2006, 128 (14), 4694-4702.

64. Cody, R. B.; Tamura, J.; Musselman, B. D., Electrospray ionization/magnetic sector mass spectrometry: calibration, resolution, and accurate mass measurements. Anal. Chem. 1992, 64 (14), 1561-1570.

65. Altieri, K.; Turpin, B.; Seitzinger, S., Oligomers, organosulfates, and nitrooxy organosulfates in rainwater identified by ultra-high resolution electrospray ionization FTICR mass spectrometry. Atmos. Chem. Phys. 2009, 9 (7), 2533-2542.

66. Mirgorodskaya, O. A.; Shevchenko, A. A.; Chernushevich, I. V.; Dodonov, A. F.; Miroshnikov, A. I., Electrospray-ionization time-of-flight mass spectrometry in protein chemistry. Anal. Chem. 1994, 66 (1), 99-107. 
67. Van Berkel, G. J.; Glish, G. L.; McLuckey, S. A., Electrospray ionization combined with ion trap mass spectrometry. Anal. Chem. 1990, 62 (13), 1284-1295.

68. Kerwin, J. L.; Tuininga, A. R.; Ericsson, L. H., Identification of molecular species of glycerophospholipids and sphingomyelin using electrospray mass spectrometry. J. Lipid Res. 1994, 35 (6), 1102-14.

69. Hsu, F.-F.; Turk, J., Structural determination of sphingomyelin by tandem mass spectrometry with electrospray ionization. J. Am. Soc. Mass. Spectrom. 2000, 11 (5), 437449.

70. Hsu, F.-F.; Turk, J., Studies on phosphatidylglycerol with triple quadrupole tandem mass spectrometry with electrospray ionization: fragmentation processes and structural characterization. J. Am. Soc. Mass. Spectrom. 2001, 12 (9), 1036-1043.

71. Hsu, F.-F.; Turk, J., Electrospray ionization/tandem quadrupole mass spectrometric studies on phosphatidylcholines: The fragmentation processes. J. Am. Soc. Mass. Spectrom. 2003, 14 (4), 352-363.

72. Hsu, F.-F.; Turk, J., Structural characterization of unsaturated glycerophospholipids by multiple-stage linear ion-trap mass spectrometry with electrospray ionization. J. Am. Soc. Mass. Spectrom. 2008, 19 (11), 1681-1691.

73. Hsu, F.-F.; Turk, J., Electrospray ionization with low-energy collisionally activated dissociation tandem mass spectrometry of glycerophospholipids: Mechanisms of fragmentation and structural characterization. J. Chromatogr. B 2009, 877 (26), 26732695.

74. Marzilli, L.; Fay, L.; Dionisi, F.; Vouros, P., Structural characterization of triacylglycerols using electrospray ionization-MS ${ }^{\mathrm{n}}$ ion-trap MS. J Amer Oil Chem Soc 2003, 80 (3), 195-202.

75. Cody, R. B.; Laramée, J. A.; Durst, H. D., Versatile new ion source for the analysis of materials in open air under ambient conditions. Anal. Chem. 2005, 77 (8), 2297-2302.

76. Chernetsova, E. S.; Morlock, G. E.; Revelsky, I. A., DART mass spectrometry and its applications in chemical analysis. Russ. Chem. Rev. 2011, 80 (3), 235-255.

77. Alberici, R. M.; Simas, R. C.; Sanvido, G. B.; Romão, W.; Lalli, P. M.; Benassi, M.; Cunha, I. B.; Eberlin, M. N., Ambient mass spectrometry: bringing MS into the "real world". Anal. Bioanal. Chem. 2010, 398 (1), 265-294.

78. Watson, J. T.; Sparkmen, O. D., Introduction to Mass Spectrometry: Instrumentation, Applications and Strategies for Data Interpretation. 4th ed.; Jonn Wiley and Sons: West Sussex, England, 2007.

79. Weston, D. J., Ambient ionization mass spectrometry: current understanding of mechanistic theory; analytical performance and application areas. Analyst 2010, 135 (4), 661-668.

80. Cody, R. B., Observation of molecular ions and analysis of nonpolar compounds with the direct analysis in real time ion source. Anal. Chem. 2008, 81 (3), 1101-1107.

81. Nilles, J. M.; Connell, T. R.; Stokes, S. T.; Dupont Durst, H., Explosives detection using direct analysis in real time (DART) mass spectrometry. Propell. Explos. Pyrot. 2010, 35 (5), 446-451.

82. Steiner, R. R.; Larson, R. L., Validation of the direct analysis in real time source for use in forensic drug screening. J. Forensic Sci. 2009, 54 (3), 617-622. 
83. Grange, A. H.; Sovocool, G. W., Detection of illicit drugs on surfaces using direct analysis in real time (DART) time-of-flight mass spectrometry. Rapid Commun. Mass Spectrom. 2011, 25 (9), 1271-1281.

84. Takáts, Z.; Wiseman, J. M.; Gologan, B.; Cooks, R. G., Mass spectrometry sampling under ambient conditions with desorption electrospray ionization. Science 2004, 306 (5695), 471-473.

85. Volny, M.; Venter, A.; Smith, S. A.; Pazzi, M.; Cooks, R. G., Surface effects and electrochemical cell capacitance in desorption electrospray ionization. Analyst 2008, 133 (4), 525-531.

86. Hu, Q.; Talaty, N.; Noll, R. J.; Cooks, R. G., Desorption electrospray ionization using an orbitrap mass spectrometer: exact mass measurements on drugs and peptides. Rapid Commun. Mass Spectrom. 2006, 20 (22), 3403-3408.

87. Bereman, M. S.; Nyadong, L.; Fernandez, F. M.; Muddiman, D. C., Direct highresolution peptide and protein analysis by desorption electrospray ionization Fourier transform ion cyclotron resonance mass spectrometry. Rapid Commun. Mass Spectrom. 2006, 20 (22), 3409-3411.

88. Myung, S.; Wiseman, J. M.; Valentine, S. J.; Takáts, Z.; Cooks, R. G.; Clemmer, D. E., Coupling Desorption Electrospray Ionization with Ion Mobility/Mass Spectrometry for Analysis of Protein Structure: Evidence for Desorption of Folded and Denatured States. The Journal of Physical Chemistry B 2006, 110 (10), 5045-5051.

89. Shin, Y.-S.; Drolet, B.; Mayer, R.; Dolence, K.; Basile, F., Desorption electrospray ionization-mass spectrometry of proteins. Anal. Chem. 2007, 79 (9), 3514-3518.

90. Leuthold, L. A.; Mandscheff, J.-F.; Fathi, M.; Giroud, C.; Augsburger, M.; Varesio, E.; Hopfgartner, G., Desorption electrospray ionization mass spectrometry: direct toxicological screening and analysis of illicit ecstasy tablets. Rapid Commun. Mass Spectrom. 2006, 20 (2), 103-110.

91. Fernández, F. M.; Cody, R. B.; Green, M. D.; Hampton, C. Y.; McGready, R.; Sengaloundeth, S.; White, N. J.; Newton, P. N., Characterization of solid counterfeit drug samples by desorption electrospray ionization and direct-analysis-in-real-time coupled to time-of-flight mass spectrometry. ChemMedChem 2006, 1 (7), 702-705.

92. Rodriguez-Cruz, S. E., Rapid analysis of controlled substances using desorption electrospray ionization mass spectrometry. Rapid Commun. Mass Spectrom. 2006, 20 (1), 53-60.

93. Takáts, Z.; Wiseman, J. M.; Cooks, R. G., Ambient mass spectrometry using desorption electrospray ionization (DESI): instrumentation, mechanisms and applications in forensics, chemistry, and biology. J. Mass Spectrom. 2005, 40 (10), 1261-1275.

94. Cotte-Rodríguez, I.; Cooks, R. G., Non-proximate detection of explosives and chemical warfare agent simulants by desorption electrospray ionization mass spectrometry. Chem. Commun. 2006, (28), 2968-2970.

95. Cotte-Rodriguez, I.; Hernandez-Soto, H.; Chen, H.; Cooks, R. G., In situ trace detection of peroxide explosives by desorption electrospray ionization and desorption atmospheric pressure chemical ionization. Anal. Chem. 2008, 80 (5), 1512-1519.

96. Ifa, D. R.; Manicke, N. E.; Dill, A. L.; Cooks, R. G., Latent fingerprint chemical imaging by mass spectrometry. Science 2008, 321 (5890), 805-805.

97. Harris, G. A.; Galhena, A. S.; Fernandez, F. M., Ambient sampling/ionization mass spectrometry: applications and current trends. Anal. Chem. 2011, 83 (12), 4508-4538. 
98. Nemes, P.; Vertes, A., Laser ablation electrospray ionization for atmospheric pressure, in vivo, and imaging mass spectrometry. Anal. Chem. 2007, 79 (21), 8098-8106.

99. Nemes, P.; Vertes, A., Laser ablation electrospray ionization for atmospheric pressure molecular imaging mass spectrometry. In Mass Spectrometry Imaging, Springer: 2010; pp 159-171.

100. Nemes, P.; Barton, A. A.; Vertes, A., Three-dimensional imaging of metabolites in tissues under ambient conditions by laser ablation electrospray ionization mass spectrometry. Anal. Chem. 2009, 81 (16), 6668-6675.

101. Nemes, P.; Woods, A. S.; Vertes, A., Simultaneous imaging of small metabolites and lipids in rat brain tissues at atmospheric pressure by laser ablation electrospray ionization mass spectrometry. Anal. Chem. 2010, 82 (3), 982-988.

102. Parsiegla, G.; Shrestha, B.; Carrière, Frederic; Vertes, A., Direct analysis of phycobilisomal antenna proteins and metabolites in small cyanobacterial populations by laser ablation electrospray ionization mass spectrometry. Anal. Chem. 2011, 84 (1), 3438 .

103. Shrestha, B.; Vertes, A., In situ metabolic profiling of single cells by laser ablation electrospray ionization mass spectrometry. Anal. Chem. 2009, 81 (20), 8265-8271.

104. March, R. E., Quadrupole ion trap mass spectrometry: A view at the turn of the century. Int. J. Mass spectrom. 2000, 200 (1-3), 285-312.

105. March, R. E., An introduction to quadrupole ion trap mass spectrometry. J. Mass Spectrom. 1997, 32 (4), 351-369.

106. March, R. E., Quadrupole ion traps. Mass Spectrom. Rev. 2009, 28 (6), 961-989.

107. March, R. E.; Todd, J. F., Quadrupole Ion Trap Mass Spectrometry. Wiley: 2005.

108. Stafford Jr, G. C.; Kelley, P. E.; Syka, J. E. P.; Reynolds, W. E.; Todd, J. F. J., Recent improvements in and analytical applications of advanced ion trap technology. Int. J. Mass Spectrom. Ion Processes 1984, 60 (1), 85-98.

109. Stafford Jr, G., Ion trap mass spectrometry: a personal perspective. J. Am. Soc. Mass. Spectrom. 2002, 13 (6), 589-596.

110. Pawliszyn, J.; Chemistry, R. S. o., Applications of Solid Phase Microextraction. Royal Society of Chemistry: 1999.

111. Hao, C.; March, R. E., A survey of recent research activity in quadrupole ion trap mass spectrometry. Int. J. Mass Spectrom. 2001, 212 (1-3), 337-357.

112. Wilm, M., Principles of electrospray ionization. Cell. Mol. Biol. 2011, 10 (7).

113. Dreisewerd, K., The desorption process in MALDI. Chem. Rev. 2003, 103 (2), 395-426.

114. March, R. E.; Hughes, R. J., Quadrupole Storage Mass Spectrometry. John Wiley and Sons: New York, 1989.

115. Hoffman, E. d.; Stroobant, V., Mass Spectrometry: Principles and Applications. 3rd ed.; John Wiley and Sons: West Sussex, England, 2007.

116. Busch, K., Space charge in mass spectrometry. Spectroscopy 2004, 19 (6), 35-38.

117. Ioanoviciu, D.; Ioanoviciu, A. S., Paul trap: axial ejection by auxiliary radio frequency voltage connection to the end-caps or by pure dipole field. Nuclear Instruments and Methods in Physics Research Section A: Accelerators, Spectrometers, Detectors and Associated Equipment 1999, 427 (1-2), 161-165.

118. Fulford, J. E.; Nhu-Hoa, D.; Hughes, R. J.; March, R. E.; Bonner, R. F.; Wong, G. J., Radio-frequency mass selective excitation and resonant ejection of ions in a three- 
dimensional quadrupole ion trap. Journal of Vacuum Science \&amp; Technology 1980, 17 (4), 829-835.

119. March, R. E.; Todd, J. F. J., Practical Aspects of Ion Trap Mass Spectrometry: Chemical, Environmental, and Biomedical Applications. Taylor \& Francis: 1995.

120. Matamoros Fernández, L. E., Introduction to ion trap mass spectrometry: Application to the structural characterization of plant oligosaccharides. Carbohydr. Polym. 2007, 68 (4), 797-807.

121. Charles, M. J.; McLuckey, S. A.; Glish, G. L., Competition between resonance ejection and ion dissociation during resonant excitation in a quadrupole ion trap. J. Am. Soc. Mass. Spectrom. 1994, 5 (12), 1031-1041.

122. Mitchell Wells, J.; McLuckey, S. A., Collision-Induced Dissociation (CID) of Peptides and Proteins. In Methods Enzymol., Burlingame, A. L., Ed. Academic Press: 2005; Vol. Volume 402, pp 148-185.

123. Kaltashov, I. A.; Eyles, S. J.; Desiderio, D. M., Mass Spectrometry in Structural Biology and Biophysics: Architecture, Dynamics, and Interaction of Biomolecules. Wiley: 2012.

124. Watson, A. D., Thematic review series: Systems biology approaches to metabolic and cardiovascular disorders. Lipidomics: A global approach to lipid analysis in biological systems. J. Lipid Res. 2006, 47 (10), 2101-2111.

125. Roberts, L. D.; McCombie, G.; Titman, C. M.; Griffin, J. L., A matter of fat: An introduction to lipidomic profiling methods. J. Chromatogr. B 2008, 871 (2), 174-181.

126. Fahy, E.; Subramaniam, S.; Alex, B. H.; Glass, C. K.; Merrill Jr., A. H.; Murphy, R. C.; Raetz, C. R. H.; Russell, D. W.; Seyama, Y.; Shaw, W.; Shimizu, T.; Spener, F.; van Meer, G.; VanNieuwenhze, M. S.; White, S. H.; Witztum, J. L.; Dennis, E. A., A comprehensive classification system for lipids. J. Lipid Res. 2005, 46, 839-861.

127. Schmelzer, K.; Fahy, E.; Subramaniam, S.; Dennis, E. A., The Lipid Maps Initiative in Lipidomics. In Methods Enzymol., Brown, H. A., Ed. Academic Press: 2007; Vol. 432, pp 171-183.

128. Adams, J.; Ann, Q., Structure determination of sphingolipids by mass spectrometry. Mass Spectrom. Rev. 1993, 12 (1), 51-85.

129. Costello, C. E.; Vath, J. E., [40] Tandem mass spectrometry of glycolipids. In Methods Enzymol., James, A. M., Ed. Academic Press: 1990; Vol. Volume 193, pp 738-768.

130. Cheng, C.; Gross, M. L.; Pittenauer, E., Complete structural elucidation of triacylglycerols by tandem sector mass spectrometry. Anal. Chem. 1998, 70 (20), 44174426.

131. McLuckey, S. A.; Goeringer, D. E., Special Feature: Tutorial slow heating methods in tandem mass spectrometry. J. Mass Spectrom. 1997, 32 (5), 461-474.

132. Blanksby, S. J.; Mitchell, T. W., Advances in mass spectrometry for lipidomics. Annu. Rev. Anal. Chem. 2010, 3 (1), 433-465.

133. Murphy, R. C.; Fiedler, J.; Hevko, J., Analysis of nonvolatile lipids by mass spectrometry. Chem. Rev. 2001, 101 (2), 479-526.

134. Ho, Y.-P.; Huang, P.-C.; Deng, K.-H., Metal ion complexes in the structural analysis of phospholipids by electrospray ionization tandem mass spectrometry. Rapid Commun. Mass Spectrom. 2003, 17 (2), 114-121.

135. Thomas, M. C.; Mitchell, T. W.; Blanksby, S. J., Ozonolysis of phospholipid double bonds during electrospray ionization: A new tool for structure determination. $J$. Am. Chem. Soc. 2006, 128 (1), 58-59. 
136. Thomas, M. C.; Mitchell, T. W.; Harman, D. G.; Deeley, J. M.; Murphy, R. C.; Blanksby, S. J., Elucidation of double bond position in unsaturated lipids by ozone electrospray ionization mass spectrometry. Anal. Chem. 2007, 79 (13), 5013-5022.

137. Thomas, M. C.; Mitchell, T. W.; Harman, D. G.; Deeley, J. M.; Nealon, J. R.; Blanksby, S. J., Ozone-induced dissociation: Elucidation of double bond position within massselected lipid ions. Anal. Chem. 2008, 80 (1), 303-311.

138. Ilchenko, S.; Cotter, R. J., Collision energetics in a tandem time-of-flight (TOF/TOF) mass spectrometer with a curved-field reflectron. Int. J. Mass spectrom. 2007, 265 (2), 372-381.

139. Medzihradszky, K. F.; Campbell, J. M.; Baldwin, M. A.; Falick, A. M.; Juhasz, P.; Vestal, M. L.; Burlingame, A. L., The characteristics of peptide collision-induced dissociation using a high-performance MALDI-TOF/TOF tandem mass spectrometer. Anal. Chem. 2000, 72 (3), 552-558.

140. Nizigiyimana, L.; Van den Heuvel, H.; Claeys, M., Comparison of low-and high-energy collision-induced dissociation tandem mass spectrometry in the analysis of ricinoleic and ricinelaidic acid. J. Mass Spectrom. 1997, 32 (3), 277-286.

141. Cheng, C.; Gross, M. L., Applications and mechanisms of charge-remote fragmentation. Mass Spectrom. Rev. 2000, 19 (6), 398-420.

142. Wysocki, V. H.; Ross, M. M., Charge-remote fragmentation of gas-phase ions: mechanistic and energetic considerations in the dissociation of long-chain functionalized alkanes and alkenes. Int. J. Mass Spectrom. Ion Processes 1991, 104 (3), 179-211.

143. Claeys, M.; Nizigiyimana, L.; Van den Heuvel, H.; Derrick, P. J., Mechanistic aspects of charge-remote fragmentation in saturated and mono-unsaturated fatty acid derivatives. Evidence for homolytic cleavage. Rapid Commun. Mass Spectrom. 1996, 10 (7), 770774.

144. Yoon, S. H.; Moon, J. H.; Choi, K. M.; Kim, M. S., A deflection system to reduce the interference from post-source decay product ions in photodissociation tandem time-offlight mass spectrometry. Rapid Commun. Mass Spectrom. 2006, 20 (14), 2201-2208.

145. Cotter, R. J., Peer Reviewed: The New Time-of-Flight Mass Spectrometry. Anal. Chem. 1999, 71 (13), 445A-451A.

146. Spengler, B.; Kirsch, D.; Kaufmann, R.; Cotter, R., Metastable decay of peptides and proteins in matrix-assisted laser-desorption mass spectrometry. Rapid Commun. Mass Spectrom. 1991, 5 (4), 198-202.

147. Zhang, X.; Jai-nhuknan, J.; Cassady, C. J., Collision-induced dissociation and post-source decay of model dodecapeptide ions containing lysine and glycine. Int. J. Mass Spectrom. Ion Processes 1997, 171 (1-3), 135-145.

148. LeRiche, T.; Osterodt, J.; Volmer, D. A., An experimental comparison of electrospray ion-trap and matrix-assisted laser desorption/ionization post-source decay mass spectra for the characterization of small drug molecules. Rapid Commun. Mass Spectrom. 2001, 15 (8), 608-614.

149. Keough, T.; Youngquist, R. S.; Lacey, M. P., A method for high-sensitivity peptide sequencing using postsource decay matrix-assisted laser desorption ionization mass spectrometry. P. Natl. Acad. Sci. USA 1999, 96 (13), 7131-7136.

150. Spengler, B., Post-source decay analysis in matrix-assisted laser desorption/ionization mass spectrometry of biomolecules. J. Mass Spectrom. 1997, 32 (10), 1019-1036. 
151. Stübiger, G.; Pittenauer, E.; Allmaier, G., MALDI seamless postsource decay fragment ion analysis of sodiated and lithiated phospholipids. Anal. Chem. 2008, 80 (5), 16641678.

152. Ham, B. M.; Jacob, J. T.; Cole, R. B., MALDI-TOF MS of phosphorylated lipids in biological fluids using immobilized metal affinity chromatography and a solid ionic crystal matrix. Anal. Chem. 2005, 77 (14), 4439-4447.

153. Al-Saad, K.; Siems, W.; Hill, H. H.; Zabrouskov, V.; Knowles, N. R., Structural analysis of phosphatidylcholines by post-source decay matrix-assisted laser desorption/ionization time-of-flight mass spectrometry. J. Am. Soc. Mass. Spectrom. 2003, 14 (4), 373-382.

154. Fuchs, B.; Schober, C.; Richter, G.; Süß, R.; Schiller, J., MALDI-TOF MS of phosphatidylethanolamines: Different adducts cause different post source decay (PSD) fragment ion spectra. J. Biochem. Bioph. Methods 2007, 70 (4), 689-692.

155. Banoub, J. H.; Limbach, P. A., Mass Spectrometry of Nucleosides and Nucleic Acids. Taylor \& Francis: 2009.

156. March, R. E.; Todd, J. F. J., Practical Aspects of Trapped Ion Mass Spectrometry: Applications of Ion Trapping Devices. Taylor \& Francis: 2009.

157. Gunawardena, H. P.; He, M.; Chrisman, P. A.; Pitteri, S. J.; Hogan, J. M.; Hodges, B. D. M.; McLuckey, S. A., Electron Transfer versus Proton Transfer in Gas-Phase Ion/Ion Reactions of Polyprotonated Peptides. J. Am. Chem. Soc. 2005, 127 (36), 12627-12639.

158. Hartmer, R. G.; Kaplan, D. A.; Stoermer, C.; Lubeck, M.; Park, M. A., Data-dependent electron transfer dissociation of large peptides and medium size proteins in a QTOF instrument on a liquid chromatography timescale. Rapid Commun. Mass Spectrom. 2009, 23 (15), 2273-2282.

159. Xia, Y.; Chrisman, P. A.; Pitteri, S. J.; Erickson, D. E.; McLuckey, S. A., Ion/Molecule Reactions of Cation Radicals Formed from Protonated Polypeptides via Gas-Phase Ion/Ion Electron Transfer. J. Am. Chem. Soc. 2006, 128 (36), 11792-11798.

160. McAlister, G. C.; Berggren, W. T.; Griep-Raming, J.; Horning, S.; Makarov, A.; Phanstiel, D.; Stafford, G.; Swaney, D. L.; Syka, J. E. P.; Zabrouskov, V.; Coon, J. J., A Proteomics Grade Electron Transfer Dissociation-Enabled Hybrid Linear Ion TrapOrbitrap Mass Spectrometer. Journal of Proteome Research 2008, 7 (8), 3127-3136.

161. Liang, X.; Liu, J.; LeBlanc, Y.; Covey, T.; Ptak, A. C.; Brenna, J. T.; McLuckey, S. A., Electron transfer dissociation of doubly sodiated glycerophosphocholine lipids. J. Am. Soc. Mass. Spectrom. 2007, 18 (10), 1783-1788.

162. Misharin, A. S.; Silivra, O. A.; Kjeldsen, F.; Zubarev, R. A., Dissociation of peptide ions by fast atom bombardment in a quadrupole ion trap. Rapid Commun. Mass Spectrom. 2005, 19 (15), 2163-2171.

163. Berkout, V. D., Fragmentation of protonated peptide ions via interaction with metastable atoms. Anal. Chem. 2006, 78 (9), 3055-3061.

164. Berkout, V. D., Fragmentation of singly protonated peptides via interaction with metastable rare gas atoms. Anal. Chem. 2008, 81 (2), 725-731.

165. Burkhardt, C. E.; Leventhal, J. J., Topics in Atomic Physics. Springer: 2006.

166. Siska, P., Molecular-beam studies of Penning ionization. Rev. Mod. Phys. 1993, 65 (2), 337.

167. Noto, J.; Kerr, R.; Shea, E.; Waldrop, L.; Fisher, G.; Rudy, R.; Hecht, J.; Gonzalez, S.; Sulzer, M.; Garcia, R., Evidence for recombination as a significant source of metastable helium. J. Geophys. Res.-Space 1998, 103 (A6), 11595-11603. 
168. Morgner, H., The characterization of liquid and solid surfaces with metastable helium atoms. In Advances In Atomic, Molecular, and Optical Physics, Benjamin, B.; Herbert, W., Eds. Academic Press: 2000; Vol. Volume 42, pp 387-488.

169. Miller, W. H., Theory of penning ionization. I. Atoms. J. Chem. Phys. 1970, 52 (7), 3563-3572.

170. Penning, F. M., Über Ionisation durch metastabile Atome. Naturwissenschaften 1927, 15 (40), 818-818.

171. Berkout, V. D; Doroshenko, V. M., Fragmentation of phosphorylated and singly charged peptide ions via interaction with metastable atoms. Int. J. Mass spectrom. 2008, 278 (23), 150-157.

172. Kishimoto, N.; Hagihara, Y.; Ohno, K.; Knippenberg, S.; François, J.-P.; Deleuze, M. S., Probing the Shape and Stereochemistry of Molecular Orbitals in Locally Flexible Aromatic Chains: A Penning Ionization Electron Spectroscopy and Green's Function Study of the Electronic Structure of Biphenyl. The Journal of Physical Chemistry A 2005, 109 (46), 10535-10546.

173. Kishimoto, N.; Osada, Y.; Ohno, K., Penning ionization of amides by collision with $\mathrm{He}^{*}(23 \mathrm{~S})$ metastable atoms. J. Electron. Spectrosc. Relat. Phenom. 2001, 114-116 (0), 183-190.

174. Madison, T. A.; Siska, P. E., Penning ionization and ion fragmentation of formamide HCONH2 by He*, Ne*, and Ar* in molecular beams. The Journal of Chemical Physics 2009, 131 (13), -.

175. Ohno, K.; Imai, K.; Harada, Y., Variations in reactivity of lone-pair electrons due to intramolecular hydrogen bonding as observed by Penning ionization electron spectroscopy. J. Am. Chem. Soc. 1985, 107 (26), 8078-8082.

176. Ohno, K.; Takano, S.; Mase, K., Penning ionization electron spectroscopy of molecules containing the $\mathrm{C}=\mathrm{O}$ group. Aldehydes and carboxylic acids. The Journal of Physical Chemistry 1986, 90 (10), 2015-2019.

177. Murphy, R. C.; Harrison, K. A., Fast atom bombardment mass spectrometry of phospholipids. Mass Spectrom. Rev. 1994, 13 (1), 57-75.

178. Ann, Q.; Adams, J., Collision-induced decomposition of sphingomyelins for structural elucidation. Biol. Mass Spectrom. 1993, 22 (5), 285-294.

179. Blackstock Wp Fau - Weir, M. P.; Weir, M. P., Proteomics: quantitative and physical mapping of cellular proteins. Tibtech 1999, 17 (0167-7799 (Print)).

180. Kevin, P. W., Functional genomics and the study of development, variation and evolution. Nature Rev. Genet. 2001, 2 (7), 528-537.

181. Wenk, M. R., The emerging field of lipidomics. Nat. Rev. Drug Discovery 2005, 4 (7), 594-610.

182. Wong, E. V., Cells: Molecules and Mechanisms. Axolotl Academic Publishing.

183. Yurlova, L.; Kahya, N.; Aggarwal, S.; Kaiser, H.-J.; Chiantia, S.; Bakhti, M.; PewznerJung, Y.; Ben-David, O.; Futerman, Anthony H.; Brügger, B.; Simons, M., Selfsegregation of myelin membrane lipids in model membranes. Biophys. J. 2011, 101 (11), 2713-2720.

184. Klein, R. A., Mass spectrometry of the phosphatidylcholines: dipalmitoyl, dioleoyl, and stearoyl-oleoyl glycerylphosphorylcholines. J. Lipid Res. 1971, 12 (2), 123-131. 
185. Furlong, S. T.; Leary, J. A.; Costello, C. E.; Dawidowicz, E. A., Isolation and identification of 1(3),2-diacylglyceryl-(3)-O-4'-(N,N,N-trimethyl)homoserine from the soil amoeba, Acanthamoeba castellanii. J. Lipid Res. 1986, 27 (11), 1182-9.

186. Klein, R. A., Mass spectrometry of the phosphatidylcholines: fragmentation processes for dioleoyl and stearoyl-oleoyl glycerylphosphorylcholine. J. Lipid Res. 1971, 12 (5), 628634.

187. Ho, Y.-P.; Huang, P.-C., A novel structural analysis of glycerophosphocholines as $\mathrm{TFA} / \mathrm{K}+$ adducts by electrospray ionization ion trap tandem mass spectrometry. Rapid Commun. Mass Spectrom. 2002, 16 (16), 1582-1589.

188. Domingues, P.; Domingues, M. R. M.; Amado, F. M. L.; Ferrer-Correia, A. J., Characterization of sodiated glycerol phosphatidylcholine phospholipids by mass spectrometry. Rapid Commun. Mass Spectrom. 2001, 15 (10), 799-804.

189. Mitchell, T. W.; Pham, H.; Thomas, M. C.; Blanksby, S. J., Identification of double bond position in lipids: From GC to OzID. J. Chromatogr. B 2009, 877 (26), 2722-2735.

190. Madsen, J. A.; Cullen, T. W.; Trent, M. S.; Brodbelt, J. S., IR and UV photodissociation as analytical tools for characterizing lipid A structures. Anal. Chem. 2011, 83 (13), 51075113.

191. Zehethofer, N.; Scior, T.; Lindner, B., Elucidation of the fragmentation pathways of different phosphatidylinositol phosphate species (PIPx) using IRMPD implemented on a FT-ICR MS. Anal. Bioanal. Chem. 2010, 398 (7-8), 2843-2851.

192. Castro-Perez, J.; Roddy, T.; Nibbering, N. M.; Shah, V.; McLaren, D.; Previs, S.; Attygalle, A.; Herath, K.; Chen, Z.; Wang, S.-P.; Mitnaul, L.; Hubbard, B.; Vreeken, R.; Johns, D.; Hankemeier, T., Localization of fatty acyl and double bond positions in phosphatidylcholines using a dual stage CID fragmentation coupled with ion mobility mass spectrometry. J. Am. Soc. Mass. Spectrom. 2011, 22 (9), 1552-1567.

193. Pasinszki, T.; Yamakado, H.; Ohno, K., Penning ionization of $\mathrm{CH}_{3} \mathrm{CN}$ and $\mathrm{CH}_{3} \mathrm{NC}$ by collision with He $\left(2^{3} \mathrm{~S}\right)$ metastable atoms. J. Phys. Chem. 1995, 99 (40), 14678-14685.

194. Kishimoto, N.; Aizawa, J.; Yamakado, H.; Ohno, K., Collision-energy-resolved penning ionization electron spectroscopy of nitriles: Conjugation effects on interactions with $\mathrm{He}^{*}\left(2^{3} \mathrm{~S}\right)$ metastable atoms. J. Phys. Chem. A 1997, 101 (28), 5038-5045.

195. Lazar, M., Free Radicals in Chemistry and Biology. Taylor \& Francis: 1989.

196. McLafferty, F., Interpretation of Mass Spectra. W.A. Benjamin: New York City, New York, 1966; p 229.

197. Jensen, N. J.; Tomer, K. B.; Gross, M. L., Gas-phase ion decomposition occurring remote to a charge site. J. Am. Chem. Soc. 1985, 107 (7), 1863-1868.

198. Levine, R. D., Molecular Reaction Dynamics. Cambridge University Press: 2005.

199. Ohno, K.; Okamura, K.; Yamakado, H.; Hoshino, S.; Takami, T.; Yamauchi, M., Penning ionization of $\mathrm{HCHO}, \mathrm{CH}_{2} \mathrm{CH}_{2}$, and $\mathrm{CH}_{2} \mathrm{CHCHO}$ by collision with $\mathrm{He}\left(2^{3} \mathrm{~S}\right)$ metastable atoms. J. Phys. Chem. 1995, 99 (39), 14247-14253.

200. Yamakado, H.; Yamauchi, M.; Hoshino, S.; Ohno, K., Penning ionization of $\mathrm{CH}_{3} \mathrm{OH},\left(\mathrm{CH}_{3}\right)_{2} \mathrm{O}$, and $(\mathrm{CH} 3 \mathrm{CH} 2) 2 \mathrm{O}$ by collision with $\mathrm{He}\left(2^{3} \mathrm{~S}\right)$ metastable atoms. $J$. Phys. Chem. 1995, 99 (47), 17093-17099.

201. Longuet-Higgins, H. C., Some Studies in Molecular Orbital Theory I. Resonance Structures and Molecular Orbitals in Unsaturated Hydrocarbons. The Journal of Chemical Physics 1950, 18 (3), 265-274. 
202. Herndon, W. C., Resonance theory and the enumeration of Kekule structures. J. Chem. Educ. 1974, 51 (1), 10.

203. Glendening, E. D.; Weinhold, F., Natural resonance theory: I. General formalism. $J$. Comput. Chem. 1998, 19 (6), 593-609.

204. Bell, S., Encyclopedia of Forensic Science. Facts On File, Incorporated: 2009.

205. O'Neal, C. L.; Crouch, D. J.; Fatah, A. A., Validation of twelve chemical spot tests for the detection of drugs of abuse. Forensic Sci. Int. 2000, 109 (3), 189-201.

206. (SWGDRUG), SWGDRUG recommendations; Washington D.C., 2007-2011; pp 1-62.

207. Bono, J. P., Drug Abuse Handbook. 2nd ed.; CRC Press: Boca Raton, FL, 2007; p 1-60.

208. Williams, T. A.; Riddle, M.; Morgan, S. L.; Brewer, W. E., Rapid gas chromatographic analysis of drugs of forensic interest. J. Chromatogr. Sci. 1999, 37 (6), 210-214.

209. Strano-Rossi, S.; Bermejo, A. M.; de la Torre, X.; Botrè, F., Fast GC-MS method for the simultaneous screening of THC-COOH, cocaine, opiates and analogues including buprenorphine and fentanyl, and their metabolites in urine. Anal. Bioanal. Chem. 2011, $399(4), 1623-1630$.

210. Overton, E. B.; Carney, K. R.; Roques, N.; Dharmasena, H. P., Fast GC instrumentation and analysis for field applications. Field Anal. Chem. Technol. 2001, 5 (1-2), 97-105.

211. Maštovská, K.; Lehotay, S. J., Practical approaches to fast gas chromatography-mass spectrometry. J. Chromatogr. A 2003, 1000 (1-2), 153-180.

212. Myung, S.; Wiseman, J. M.; Valentine, S. J.; Takáts, Z.; Cooks, R. G.; Clemmer, D. E., Coupling desorption electrospray ionization with ion mobility/mass spectrometry for analysis of protein structure: Evidence for desorption of folded and denatured states. $J$. Phys. Chem. B 2006, 110 (10), 5045-5051.

213. Talaty, N.; Takáts, Z.; Cooks, R. G., Rapid in situ detection of alkaloids in plant tissue under ambient conditions using desorption electrospray ionization. Analyst 2005, 130 (12), 1624-1633.

214. Duvivier, W. F.; van Beek, T. A.; Pennings, E. J. M.; Nielen, M. W. F., Rapid analysis of $\Delta$-9-tetrahydrocannabinol in hair using direct analysis in real time ambient ionization orbitrap mass spectrometry. Rapid Commun. Mass Spectrom. 2014, 28 (7), 682-690.

215. Huang, M.-Z.; Cheng, S.-C.; Cho, Y.-T.; Shiea, J., Ambient ionization mass spectrometry: a tutorial. Anal. Chim. Acta 2011, 702 (1), 1-15.

216. Moeller, K. E.; Lee, K. C.; Kissack, J. C. In Urine drug screening: practical guide for clinicians, Mayo Clinic Proceedings, Elsevier: 2008; pp 66-76.

217. Mikkelsen, S.; Ash, K., Adulterants causing false negatives in illicit drug testing. Clin. Chem. 1988, 34 (11), 2333-2336.

218. Wu, C.; Dill, A. L.; Eberlin, L. S.; Cooks, R. G.; Ifa, D. R., Mass spectrometry imaging under ambient conditions. Mass Spectrom. Rev. 2012.

219. Srinivas, M.; Hopperstad, M. G.; Spray, D. C., Quinine blocks specific gap junction channel subtypes. Proc. Natl. Acad. Sci. USA 2001, 98 (19), 10942-10947.

220. Horning, M. G.; Brown, L.; Nowlin, J.; Lertratanangkoon, K.; Kellaway, P.; Zion, T. E., Use of saliva in therapeutic drug monitoring. Clin. Chem. 1977, 23 (2), 157-164.

221. O'Neal, C. L.; Crouch, D. J.; Rollins, D. E.; Fatah, A.; Cheever, M. L., Correlation of saliva codeine concentrations with plasma concentrations after oral codeine administration. J. Anal. Toxicol. 1999, 23 (6), 452-459.

222. Al-Ghananeem, A. M.; Malkawi, A. H.; Crooks, P. A., Effect of $\mathrm{pH}$ on sublingual absorption of oxycodone hydrochloride. AAPS PharmsSciTech 2006, 7 (1). 
223. Paulsen, R. B.; Wilkins, D. G.; Slawson, M. H.; Shaw, K.; Rollins, D. E., Effect of four laboratory decontamination procedures on the quantitative determination of cocaine and metabolites in hair by HPLC-MS. J. Anal. Toxicol. 2001, 25 (7), 490-496.

224. Peltenburg, H.; Groothuis, F. A.; Droge, S. T. J.; Bosman, I. J.; Hermens, J. L. M., Elucidating the sorption mechanism of "mixed-mode" SPME using the basic drug amphetamine as a model compound. Anal. Chim. Acta 2013, 782, 21-27.

225. Logan, B., Methamphetamine-effects on human performance and behavior. Forensic Sci. Rev. 2002, 14 (1), 133-151.

226. Zuo, Y.; Zhang, K.; Wu, J.; Rego, C.; Fritz, J., An accurate and nondestructive GC method for determination of cocaine on US paper currency. J. Sep. Sci. 2008, 31 (13), 2444-2450.

227. Bhatt, P. M.; Ravindra, N. V.; Banerjee, R.; Desiraju, G. R., Saccharin as a salt former. Enhanced solubilities of saccharinates of active pharmaceutical ingredients. Chem. Commun. 2005, (8), 1073-1075.

228. Stein, S. E.; Pierre, A.; Lias, S. G., Comparative evaluations of mass spectral databases. J. Am. Soc. Mass. Spectrom. 1991, 2 (5), 441-443.

229. Demuth, W.; Karlovits, M.; Varmuza, K., Spectral similarity versus structural similarity: mass spectrometry. Anal. Chim. Acta 2004, 516 (1-2), 75-85.

230. Sawada, Y.; Nakabayashi, R.; Yamada, Y.; Suzuki, M.; Sato, M.; Sakata, A.; Akiyama, K.; Sakurai, T.; Matsuda, F.; Aoki, T.; Hirai, M. Y.; Saito, K., RIKEN tandem mass spectral database (ReSpect) for phytochemicals: A plant-specific MS/MS-based data resource and database. Phytochemistry 2012, 82 (0), 38-45.

231. Kauppila, T. J.; Arvola, V.; Haapala, M.; Pól, J.; Aalberg, L.; Saarela, V.; Franssila, S.; Kotiaho, T.; Kostiainen, R., Direct analysis of illicit drugs by desorption atmospheric pressure photoionization. Rapid Commun. Mass Spectrom. 2008, 22 (7), 979-985.

232. Rodriguez-Cruz, S. E., Rapid screening of seized drug exhibits using desorption electrospray ionization mass spectrometry (DESI-MS). Microgram J. 2008, 6 (1-2), 1025.

233. Khan, J.V.I; Kennedy, T.J.; Christian, D.R., Basic Principles of Forensic Chemistry. Humana Press: 2011.

234. Oguri, K.; Wada, S.; Eto, S.; Yamada, H., Specificity and Mechanism of the Color Reaction of Cocaine with Cobaltous Thiocyanate. Jpn. J. Toxicol. Environ. Health 1995, 41 (4), 274-279.

235. Wainhaus, S. B.; Tzanani, N.; Dagan, S.; Miller, M. L.; Amirav, A., Fast analysis of drugs in a single hair. J. Am. Soc. Mass. Spectrom. 1998, 9 (12), 1311-1320.

236. Miki, A.; Katagi, M.; Kamata, T.; Zaitsu, K.; Tatsuno, M.; Nakanishi, T.; Tsuchihashi, H.; Takubo, T.; Suzuki, K., MALDI-TOF and MALDI-FTICR imaging mass spectrometry of methamphetamine incorporated into hair. J. Mass Spectrom. 2011, 46 (4), 411-416.

237. Günther, D.; Jackson, S. E.; Longerich, H. P., Laser ablation and arc/spark solid sample introduction into inductively coupled plasma mass spectrometers. Spectrochim. Acta B 1999, 54 (3-4), 381-409. 
